\title{
THERMAL SPRINGS LIST FOR THE UNITED STATES

National Oceanic and Atmospheric Administration Key to Geophysical Records Documentation No. 12

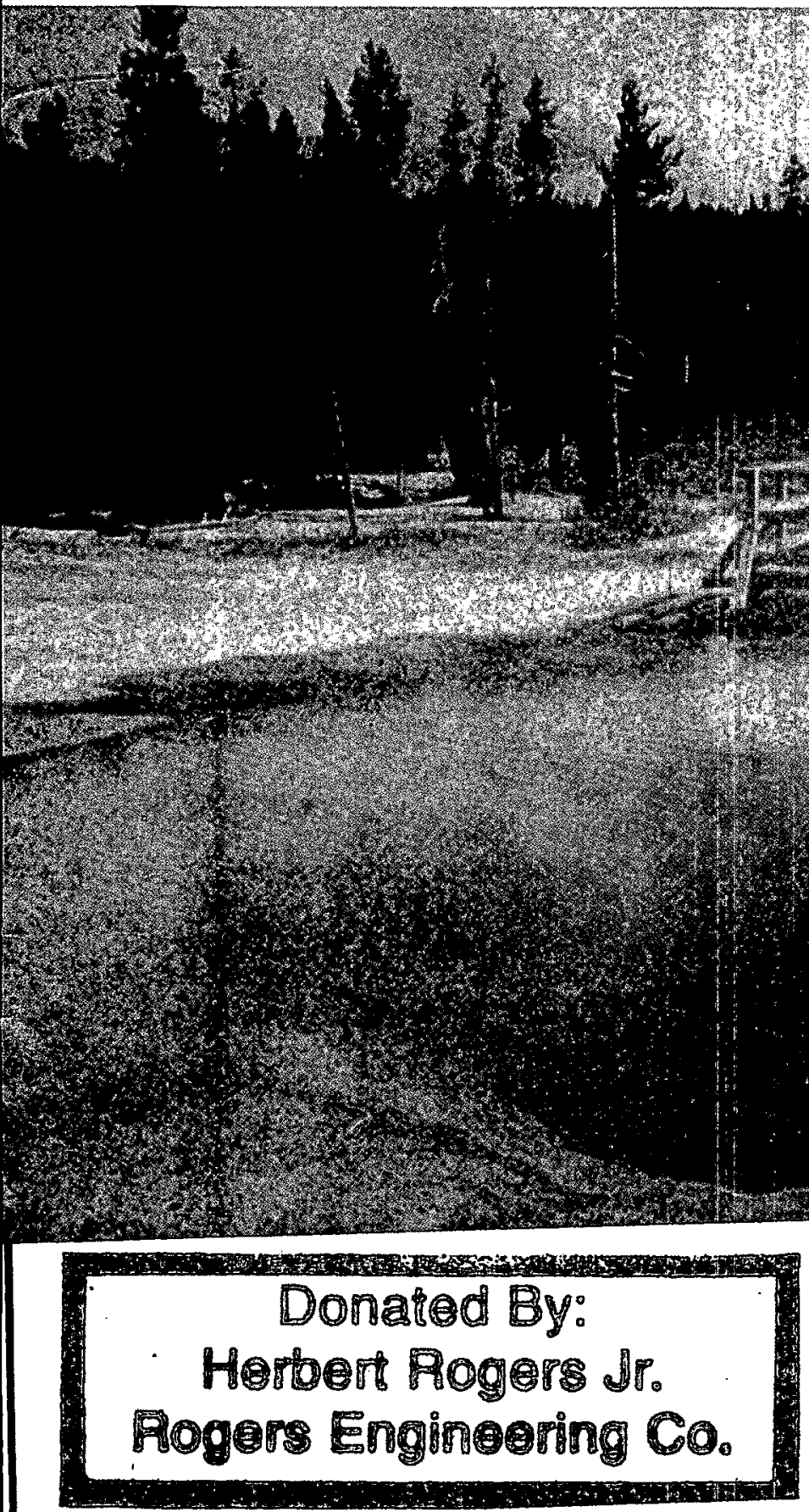

U.S. Department of Commerce

National Oceanic and Atmospheric Administration Environmental Data and Inlormation Service

National Geophysical and Solar-Terrestrial Data Center Boulder, Colorado

June 1980

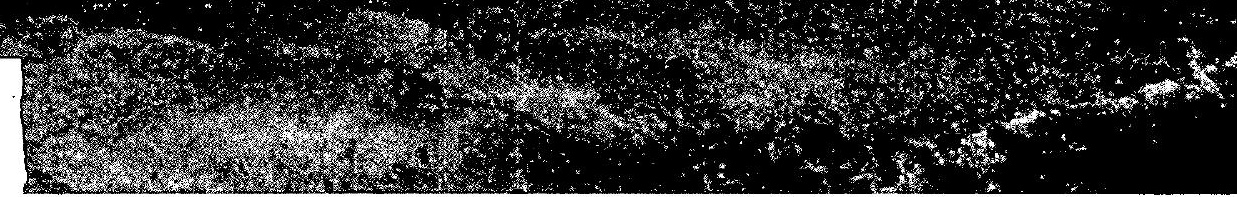

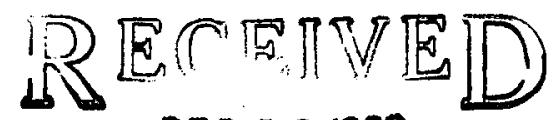

\section{DEC 221980}

Rogrers Engineering $\mathrm{CO}_{1}$ Inc. ON FANCIBCO 


\section{DISCLAIMER}

This report was prepared as an account of work sponsored by an agency of the United States Government. Neither the United States Government nor any agency Thereof, nor any of their employees, makes any warranty, express or implied, or assumes any legal liability or responsibility for the accuracy, completeness, or usefulness of any information, apparatus, product, or process disclosed, or represents that its use would not infringe privately owned rights. Reference herein to any specific commercial product, process, or service by trade name, trademark, manufacturer, or otherwise does not necessarily constitute or imply its endorsement, recommendation, or favoring by the United States Government or any agency thereof. The views and opinions of authors expressed herein do not necessarily state or reflect those of the United States Government or any agency thereof. 


\section{DISCLAIMER}

Portions of this document may be illegible in electronic image products. Images are produced from the best available original document. 
Listed below are catalogs published in the Key to Geophysical Records Documentation (KGRD) series. Those without an asterisk $\left(^{*}\right)$ may be obtained from National Geophysical and Solar-Terrestrial Data Center, NOAAEDIS, Boulder, Colorado, USA, 80303; if preceded by an asterisk, from National Technical Information Service, U.S. Department of Commerce, Springfield, Virginia, USA, 22161.

KGRD No. 1, Marine Geophysical Data Catalog (superseded by KGRD No. 4).

*KGRD No. 2, Catalog of Strong-Motion Seismograph Stations and Records (COM-74-10714).

KGRD No. 3, Catalog of Earthquake Photographs (superseded by KGRD No. 11).

KGRD No. 4, Marine Geophysical Data Catalog-1975 (superseded by KGRD No. 11).

KGRD No. 5, Earthquake Data File Summary.

*KGRD No. 6, Bibliography and Index to Literature on Manganese Nodules (1874-1975).

KGRD No. 7, Catalog of Earthquake Photographs.

KGRD No. 8, Catalog of Digital Bathymetric Data for the United States Coastal Regions.

KGRD No. 9, Catalog of Seismogram Archives.

KGRD No. 10, The Marine Geophysical Data Exchange Format-"MCD77".

KGRD No. 11, Summary of Digital Marine Geophysical Data Holdings (Bathymetric, Magnetic, and Gravimetric Data).

Additional information on the services of the National Geophysical and Solar-Terrestrial Data Center is available from the address above. The following publications describe the services for geophysics and provide price lists:

Earthquake Data Services and Publications

Marine Geology and Geophysics Data Services and Publications

Geomagnetism (Solid Earth) Data Services and Publications.

\section{Cover photo: MORNING GLORY POOL, Yellowstone National Park. Courtesy of Wyoming Travel Commission.}




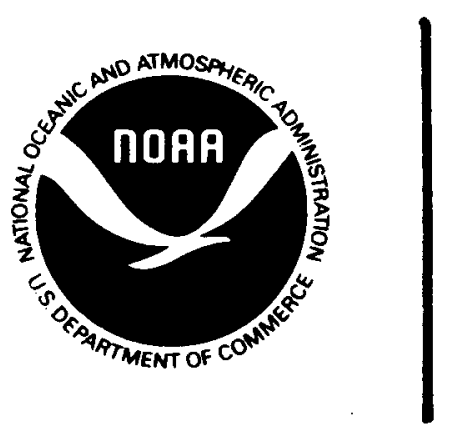

U.S. DEPARTMENT OF COMMERCE

Philip M. Klutznick, Secretary

NATIONAL OCEANIC AND ATMOSPHERIC ADMINISTRATION

Richard A. Frank, Administrator

ENVIRONMENTAL DATA AND INFORMATION SERVICE

Thomas D. Potter, Director

\title{
THERMAL SPRINGS LIST FOR THE UNITED STATES
}

National Oceanic and Atmospheric Administration Key to Geophysical Records Documentation No. 12

\author{
Compiled by
}

George W. Berry

Cooperative Institute for Research in Environmental Sciences (CIRES). University of Colorado/NOAA, Boulder, Colorado

Paul J. Grim and Joy A. Ikelman

National Geophysical and Solar-Terrestrial Data Center, NOAA, Boulder, Colorado

COPIES OF THIS PUBLICATION (INCLUDING MAPS) ARE AVAILABLE FREE OF CHARGE FROM:

NOAA/NGSDC DATAMAPPING GROUP, CODE D64, 325 BROADWAY, BOULDER, COLORADO 80303

National Geophysical and Solar-Terrestrial Data Center

Boulder, Colorado

June 1980 
This list of United States thermal spring locations and temperatures and accompanying maps have been compiled in the course of preparing the maps: (1) Ceothermal Energy Resources of the Western United States by National Geophysical and Solar-Terrestrial Data Center, National Oceanic and Atmospheric Administration (NOAA) (Grim, 1977); (2) Ceothermal Energy in the Western United States and Geothermal Energy in Alaska and Hawaii in the U.S. Geological Survey (USGS) Assessment of Ceothermal Resources of the United States-1978 (Muffler, 1979); and (3) State maps in press and preparation for the State Coupled Resource Assessment Program, U.S. Department of Energy, Division of Geothermal Energy (DOE/DGE). The list is a compilation of compilations, mainly from Waring's (1965) monumental Thermal Springs of the United States and Other Countries of the World; a Summary, USGS CEOTHERM computer file of thermal spring and well data, and currently active DOE/DGE-sponsored thermai data collection by State agencies and other organizations.

The compilation (table 1) has 1.702 thermal spring locations in 23 of the 50 States, arranged alphabetically by State (Postal Service abbreviation) and degrees of latitude and longitude within the State. It shows spring name; surface temperature in degrees Fahrenheit and degrees Celsius; USCS Professional Paper 492 number; USGS Circular 790 number; NOAA number, north to south on each degree of latitude and longitude of the listed USCS 1:250,000-scale (AMS) map; and the USCS topographic map coverage, 1:63360- or 1:62500-scale (15-minute) or 1:24000-scale (7.5-minute) quadrangle.

Table 2 is an alphabetized list showing only the spring name and the State in which it is located. Unnamed springs are omitted.

The list includes natural surface hydrothermal features: springs, pools, mud pots, mud volcanoes, geysers, fumaroles, and steam vents at temperatures of $20^{\circ} \mathrm{C}\left(68^{\circ} \mathrm{F}\right)$ or greater. It does not include wells or mines, except at sites where they supplement or replace natural vents presently or recently active, or, in some places, where orifices are not distinguishable as natural or artificial.

Many thermal localities have several names in the literature, on maps, and in local use, and within groups there are commonly several more names for the orifices. We have generalized thermal features in an area of one-half mile $(0.8 \mathrm{~km})$ dimension as a single site. Names are from USGS topographic maps, if shown, with some expansions of those names, and additional ones, from recent USCS CEOTHERM and State agency lists. "Unnamed" springs are left unnamed. We introduce no new terminology.

For most thermal sites a range of temperatures is available for separate orifices and different times. We list, in ${ }^{\circ} \mathrm{F}$ and ${ }^{\circ} \mathrm{C}$, the most recently reported maximum surface temperature. For some features we have only "boiling," "hot," or "warm." Measured subsurface temperatures in spring systems, wells, and mines, and temperatures estimated by geothermometry, are not listed.

We have located the listed springs on the USGS 1:250,000 (AMS) topographic maps shown on map 1 and map 2 (in pocket). For information on availability of overlays of these 1:250,000 maps (see map 3 in pocket as sample) on stable-base film or on paper, and availability of the springs list in a digital format, write or telephone:

NOAA/NGSDC

Datamapping Group, Code D64

325 Broadway

Boulder, Colorado 80303

Phone: (303) 497-6418 or FTS 320-6418

Under Topographic Quadrangle we list the largest scale USGS coverage by 15-minute (1:63360 or 1:62500) or 7.5-minute $(1: 24000)$ quadrangle, if the map is available. Parentheses indicate that the site is not specifically shown on the map by name or symbol.

\section{ACKNOWLEDGMENTS}

We are very grateful to the many contributors to this compilation. James R. Swanson, U.S. Ceological Survey, Menlo Park, California, graciously and efficiently provided GEOTHERM data. Sharon L. Axelson and Lawrence W. Miller, Jr. (NOAA) made the overlays (map 3 ) of the 1:250,000-scale maps. Ronald H. Smith, Cooperative Institute for Research in Environmental Sciences (CIRES), University of Colorado/NOAA, Boulder, Colorado, produced maps 1 and 2. The U.S. Department of Energy, Division of Ceothermal Energy provided financial assistance. Personal communications of important reviews, corrections, additions, deletions, and encouragement were contributed by Joe $\mathrm{H}$. Allen, NOAA, Boulder, Colorado; Gerald P. Brophy, DOE/DGE, Washington, D.C.; Leland L. Mink and Clayton R. Nichols, DOE/DGE, Idaho Falls, Idaho; Duncan Foley and Phillip M. Wright, Earth Science Laboratory/University of Utah Research Institute, Salt Lake City, Utah; Anne L. Berry, Charles A. Brook, Robert H. Mariner, L. J. P. Muffler, Marshall J. Reed, Edward A. Sammel, and Donald E. White, U.S. Ceological Survey, Menio Park, California; and:

Alaska: $\quad$ Mary Moorman and Roman J. Motyka, Alaska Division of Geological \& Geophysical Surveys, College; Ross G. Schaff, Alaska Division of Geological \& Geophysical Surveys, Anchorage; Donald L. Turner, University of Alaska Geophysical Institute, Fairbanks.

Appalachians: William A. Hobba, Jr., U.S. Geological Survey, Morgantown, W. Va.; Joel L. Renner, Gruy Federal, Inc., Arlington, Va. 
Arizona: $\quad$ W. Richard Hahman, Sr., Claudia Stone, and James C. Witcher, Arizona Bureau of Geology and Mineral Technology, Geological Survey Branch, Tucson.

California: $\quad$ Robert L. Christiansen, U.S. Geological Survey, Menlo Park; Chris T. Higgins and Roger Martin, California Division of Mines and Geology, Sacramento; Charles W. Jennings, California Division of Mines and Ceology, San Francisco

Colorado: $\quad$ Richard H. Pearl, Colorado Geological Survey, Denver.

Hawaii: Donald M. Thomas, Hawaii Institute of Geophysics, Honolulu.

Idaho:

John C. Bond, consulting geologist, Moscow; John C. Mitchell, Idaho Department of Water Resources, Boise.

Montana:

John R. Fanshawe, consulting geologist, Billings; John Sonderegger, Montana Bureau of Mines and Ceology. Butte.

Nevada: $\quad$ Andrew G. Alpha, consulting geologist, Denver, Colo.; Larry J. Garside and Dennis I. Trexler, Nevada Bureau of Mines and Ceology, Reno; Richard K. Hose, U.S. Geological Survey, Menlo Park, Calif.

New Mexico Chandler A. Swanberg, New Mexico State University, Las Cruces.

Oregon:

Richard G. Bowen, consulting geologist, Portland; Donald A. Hull and Joseph F. Riccio, Oregon Department of Geology and Mineral Industries, Portland.

South Dakota: J. Paul Gries, South Dakota School of Mines \& Technology, Rapid City.

Texas: $\quad$ Christopher D. Henry, Texas Bureau of Economic Ceology, Austin.

Utah: Harry D. Coode, consulting geologist, Salt Lake City; Peter J. Murphy, Utah Geological and Mineral Survey, Salt Lake City.

Washington: Michael Korosec and J. Eric Schuster, Washington Department of Natural Resources, Division of Geology, Olympia.

Wyoming: $\quad$ Roy M. Breckenridge, Wyoming Geological Survey, Laramie (now at Idaho Bureau of Mines and Geology, Moscow); Edward R. Decker and Henry P. Heasler, University of Wyoming, Laramie; J.D. Love, U.S. Geological Survey, Laramie.

Yellowstone

National Park

Wayne L. Hamilton and Roderick A. Hutchinson, U.S. National Park Service, Yellowstone National Park.

\section{PRINCIPAL PUBLISHED AND OPEN-FILE SOURCES}

General

Grim, P.J., 1977. Geothermal energy resources of the western United States, National Geophysical and Solar-Terrestrial Data Center, National Oceanic and Atmospheric Administration map, scale 1:2,500,000.

Muffler, L.J.P., ed., 1979. Assessment of geothermal resources of the United States-1978, U.S. Geological Survey Circular $790,163 \mathrm{pp}$.

Renner, J.L., and T.L. Vaught, 1979. Geothermal resources of the eastern United States, National Technical Information Service DOE/NVO/1558-7, $59 \mathrm{pp}$.

U.S. Geological Survey CEOTHERM file, Menlo Park, Calif.

Waring, G.A., 1965. Thermal springs of the United States and other countries of the world-a summary, U.S. Geological Survey Professional Paper 492, $383 \mathrm{pp}$

White, D. E. and D. L. Williams, eds., 1975. Assessment of geothermal resources of the United States - 1975, U.S. Ceological Survey Circular 726, $155 \mathrm{pp}$

Alaska

Conwell, C.N., and L.C. Schell, compilers, 1979. Energy resource map of Alaska, Alaska Division of Geological \& Ceophysical Surveys, scale 1:2,500,000

Markle, D.R., 1979. Ceothermal energy in Alaska: site data base and development status, Geo-Heat Utilization Center, Klamath Falls, Oreg., Prepared for U.S. Department of Energy, San Francisco Operations Office, 572 pp.

Miller, T.P., Ivan Barnes, and W.W. Patton, Jr., 1975. Ceologic setting and chemical characteristics of hot springs in westcentral Alaska, U.S. Geological Survey Journal of Research, v. 3, no. 2, pp. 149-162.

Miller, T.P., compiler, 1973. Distribution and chemical analyses of thermal springs in Alaska, U.S. Geological Survey Open-

File Map. Anchorage, Alaska, scale 1:2,500,000

Waring, G.A., 1917. Mineral springs of Alaska, U.S. Geological Survey Water-Supply Paper 418,114 pp.

Appalachians

Hobba, W.A., Jr., D.W. Fisher, F.J. Pearson, Jr., and J.C. Chemerys, 1979. Hydrology and geochemistry of thermal springs of the Appalachians, U.S. Geological Survey Professional Paper 1044-E, $36 \mathrm{pp}$ 


\section{Arizona}

Hahman, W.R., Sr., C. Stone, and J.C. Witcher, compilers, 1978. Preliminary map, geothermal energy resources of Arizona, Arizona Bureau of Ceology and Mineral Technology, Geological Survey Branch, Geothermal Map 1, scale 1:1,000,000

Mariner, R.H., T.S. Presser, and W.C. Evans, 1977. Chemical, isotopic, and gas compositions of selected thermal springs in Arizona, New Mexico, and Utah, U.S. Geological Survey Open-File Report 77-654, Menlo Park, Calif., 42 pp.

Norton, D., T. Gerlach, K.J. DeCook, and J.S. Sumner, 1975. Geothermal water resources in Arizona: feasibility study, Arizona University Technical Completion Report, Office of Water Research and Technology Project A-054-ARIZ, 41 pp.

Swanberg, C.A., Paul Morgan, C.H. Stoyer, and J.C. Witcher, 1977. An appraisal study of the geothermal resources of Arizona and adjacent areas in New Mexico and Utah and their value for desalination and other uses, New Mexico Energy Institute Technical Completion Report, $76 \mathrm{pp}$

Arkansas

Bedinger, M.S., F.J. Pearson, Jr., J.E. Reed, R.T. Sniegocki, and C.G. Stone, 1979. The waters of Hot Springs National Park, Arkansas - their nature and origin, U.S. Geological Survey Professional Paper 1044-C, 33 pp

Bryan, Kirk, 1924. The hot springs of Arkansas, Journal of Geologv, v. 32, pp. 449-459.

California

Allen, E.T., and A.L. Day, 1927. Steam wells and other thermal activity at "The Ceysers," California, Carnegie Institution of Washington Publication 378, $106 \mathrm{pp}$.

Berkstresser, C.F., Jr., 1968. Data for springs in the southern Coast, Transverse, and Peninsular Ranges of California, U.S. Geological Survey Open-File Report, Menlo Park, Calif., 21 pp.

Berkstresser, C.F., Jr., 1968. Data for springs in the northern Coast Ranges and Klamath Mountains of California, U.S. Geological Survey Open-File Report, Menlo Park, Calif., 49 pp.

Berkstresser, C.F., Jr., 1969. Data for springs in the Colorado Desert area of California, U.S. Geological Survey Open-File Report, Menlo Park, Calif., 13 pp.

California Division of Mines and Geology, 1980. Ceothermal resources of California map, scale 1:750,000 [in press].

Day, A.L., and E.T. Allen, 1925. The volcanic activity and hot springs of Lassen Peak, Carnegie Institution of Washington Publication $360,190 \mathrm{pp}$.

Duffield, W.A., and R.O. Fournier, 1974. Reconnaissance study of the geothermal resources of Modoc County, California,

U.S. Geological Survey Open-File Report, Menlo Park, Calif., 19 pp.

Hannah, J.L., 1975. Low temperature geothermal resources in northern California, California Division of Oil and Cas Report TR13, 53 pp.

Jennings, C.W., 1975. Fault map of California with locations of volcanoes, thermal springs and therma/ wells, California Division of Mines and Geology Geologic Data Map Series Map 1, scale 1:750,000.

Lewis, R.E., 1974. Data on wells, springs, and thermal springs in Long Valley, Mono County, California, U.S. Geological Survey

Open-File Report, Menlo Park, Calif., 52 pp.

Mariner, R.H., T.S. Presser, and W.C. Evans, 1976. Chemical composition data and calculated aquifer temperature for selected wells and springs of Honey Lake Valley, California, U.S. Geological Survey Open-File Report 76-783, Menlo Park, Calif., $10 \mathrm{pp}$

Mariner, R.H., T.S. Presser, and W.C. Evans, 1977. Hot springs of the central Sierra Nevada, California, U.S. Geological Survey

Open-File Report 77-559, Menlo Park, Calif., 27 pp.

Moyle, W.R., Jr., 1974. Temperature and chemical data for selected thermal wells and springs in southeastern California, U.S

Geological Survey Water-Resources Investigations 33-73, Menlo Park, Calif., 12 pp

Sims, J.D., and M. J. Rymer, 1976. Map of gaseous springs and associated faults in Clear Lake, California, U.S. Geological Survey Miscellaneous Field Studies Map MF-721, scale 1:48,000

Sorey, M.L., and R.E. Lewis, 1976. Convective heat flow from hot springs in the Long Valley caldera, Mono County, California, Journal of Geophysical Research, v. 81, pp. 785-791

Thompson, J.M. F.E. Goff, and J.M. Donnelly, 1978. Chemical analyses of waters from springs and wells from the Clear Lake volcanic area, northern California, U.S. Geological Survey Open-File Report 78-425, Menlo Park, Calif., 12 pp

White, D.E., Ivan Barnes, and J.R. O'Neil, 1973. Thermal and mineral waters of nonmeteoric origin, California Coast Ranges, Geological Society of America Bulletin, v. 84, pp. 547-560.

\section{Colorado}

Barrett, J.K., and R.H. Pearl, 1978. An appraisal of Colorado's geothermal resources, Colorado Ceological Survey Bulletin 39, $224 \mathrm{pp}$.

Pearl, R.H., compiler, 1980. Ceothermal resources of Colorado, Colorado Geological Survey Map Series 14, scale 1:500,000. [in press].

U.S. Geological Survey and Colorado Geological Survey, 1977. Energy resources map of Colorado, U.S. Geological Survey Miscellaneous Investigations Series Map 1-1039, scale 1:500,000. 
Florida

Rosenau, J.C., G.L. Faulkner, C.W. Hendry, Jr., and R.W. Hull, 1977. Springs of Florida, Florida Bureau of Ceology Bulletin 31 (Revised), $461 \mathrm{pp}$.

Georgia

Hewett, D.F., and G.W. Crickmay, 1937. The warm springs of Ceorgia, their geologic relations and origin, U.S. Geological Survey Water-Supply Paper $819,40 \mathrm{pp}$

Idaho

Mitchell, J.C., L.L. Johnson, and I.E. Anderson, 1980. Geothermal investigations in Idaho, Part 9, Potential in direct heat application of geothermal resources, Idaho Department of Water Resources Water Information Bulletin 30. [in press].

Montana

Chadwick, R.A., and R.B. Leonard, 1979. Structural controls of hot-spring systems in southwestern Montana, U.S. Geological Survey Open-File Report 79-1333, Helena, Mont., 25 pp

Leonard, R.B., T.M. Brosten, and N.A. Midtlyng, 1978. Selected data from thermal-spring areas, southwestern Montana, U.S Geological Survey Open-File Report 78-438, Helena, Mont., 71 pp.

Mariner, R.H., T.S. Presser, and W.C. Evans, 1976. Chemical characteristics of the major thermal springs of Montana, U.S Geological Survey Open-File Report 76-480, Menlo Park, Calif., 31 pp.

Nevada

Garside, L.J., and J.H. Schilling, 1979. Therma/ waters of Nevada, Nevada Bureau of Mines and Geology Bulletin 91, 163 pp.

Hose, R.K., and B.E. Taylor, 1974. Geothermal systems of northern Nevada, U.S. Geologic Survey Open-File Report 74-271, Menlo Park, Calif., 27 pp.

Mariner, R.H., J.B. Rapp, L.M. Willey, and T.S. Presser, 1974. The chemical composition and estimated minimum thermal reservoir temperatures of the principal hot springs of northern and central Nevada, U.S. Geological Survey Open-File Report, Menlo Park, Calif., 32 pp.

Olmsted, F.H., P.A. Glancy, J.R. Harrill, F.E. Rush, and A.S. Vandenburgh, 1975. Preliminary hydrologic appraisal of selected hydrothermal systems in northern and central Nevada, U.S. Geological Survey Open-File Report 75-56, Menlo Park, Calif., $267 \mathrm{pp}$.

Trexler, D.T., B.A. Koenig, and Thomas Flynn, compilers, 1979. Geothermal resources of Nevada and their potential for direct utilization, Nevada Bureau of Mines and Geology map, scale 1:500,000.

New Mexico

Summers, W.K., 1976. Catalog of thermal waters in New Mexico, New Mexico Bureau of Mines \& Mineral Resources Hydrologic Report 4, $80 \mathrm{pp}$

Swanberg, C.A., compiler, 1980. Geothermal resources of New Mexico, New Mexico Energy Institute map, scale 1:500,000. [in press].

Swanberg, C.A., Paul Morgan, C.H. Stoyer, and J.C. Witcher, 1977. An appraisal study of the geothermal resources of Arizona and adiacent areas in New Mexico and Utah and their value for desalination and other uses, New Mexico Energy Institute Technical Completion Report, $76 \mathrm{pp}$.

Oregon

Bowen, R.G., N.V. Peterson, and J.F. Riccio, 1978. Low- to intermediate-temperature thermal springs and wells in Oregon, Oregon Department of Ceology and Mineral Industries Geological Map Series CMS-10, scale 1:1,000,000.

Riccio, J.F., compiler, 1978. Preliminary geothermal resource map of Oregon, Oregon Department of Ceology and Mineral Industries Ceological Map Series GMS-11, scale 1:500,000.

South Dakota

Rahn, P.H., and J.P. Gries, 1973. Large springs in the Black Hills, South Dakota and Wyoming, South Dakota Geological Survey Report of Investigations $107,46 \mathrm{pp}$

Texas

Henry, C.D., 1979. Geologic setting and geochemistry of thermal water and geothermal assessment, Trans-Pecos Texas, Bureau of Economic Geology, University of Texas at Austin, Report of Investigations 96, 48 pp. 
Utah

Goode, H.D., 1978. Thermal waters of Utah, Utah Geological and Mineral Survey Report of Investigation 129, $183 \mathrm{pp}$.

Mundorff, J.C., 1970. Major thermal springs of Utah, Utah Geological and Mineralogical Survey Water-Resources Bulletin $13,60 \mathrm{pp}$

Utah Geological and Mineral Survey, 1977. Energy resources map of Utah, Map 44, scale 1:500,000.

Utah Geological and Mineral Survey, 1980. Ceothermal resources of Utah map, scale 1:500,000. [in press].

Virginia

Reeves, Frank, 1932. Thermal springs of Virginia, Virginia Ceological Survey Bulletin 36, $56 \mathrm{pp}$.

\section{Washington}

Campbell, K. V., J. H. Miers, B. M. Nichols, Jerrelyn Oliphant, Shirley Pytlak, R. W. Race, G.H. Shaw, and R. L. Gresens, A survey of thermal springs in Washington State, Northwest Science, v. 44, pp. 1-11.

Schuster, J.E., 1974. Ceothermal energy potential of Washington, in Energy resources of Washington, Washington Division of Geology and Earth Resources Information Circular 50, pp. 5-19.

West Virginia

Price, P.H., J.B. McCue, and H.A. Hoskins, 1936. Springs of West Virginia, West Virginia Geological Survey Reports, V. 6, $146 \mathrm{pp}$.

Erskine, H.M., 1948. Principal springs of West Virginia, a report on the location, discharge and temperature of the principle springs of West Virginia, Conservation Commission of West Virginia, $50 \mathrm{pp}$

Wroming

Breckenridge, R.M., and B.S. Hinckley, 1978. Thermal springs of Wyoming, Geological Survey of Wyoming Bulletin 60 , $104 \mathrm{pp}$.

Glass, G.B., W.G. Wendell, F.K. Root, and R.M. Breckenridge, 1975. Energy resources map of Wyoming, Geological Survey of Wyoming, scale 1:500,000

Yellowstone National Park

Allen, E.T., and A.L. Day, 1935. Hot springs of the Yellowstone National Park, Carnegie Institution of Washington Publication $466,525 \mathrm{pp}$.

Rowe, J.J., R.O. Fournier, and G.W. Morey, 1973. Chemical analysis of thermal waters in Yellowstone National Park, Wyoming, 1960-65, U.S. Geological Survey Bulletin 1303, 31 pp.

Thompson, J.M., and Sandhya Yadav, 1979. Chemical analyses of waters from geysers, hot springs and pools in Yellowstone National Park, Wyoming from 1974 to 1978, U.S. Geological Survey Open-File Report 79-704, Menlo Park, Calif., 11 pp.

Thompson, J.M., T.S. Presser, R.B. Barnes, and D.B. Bird, 1975. Chemical analysis of the waters of Yellowstone National Park, Wyoming from 1965-1973, U.S. Geological Survey Open-File Report 75-25, 59 pp. 
Table 1. Thermal springs list for the United States

Temperatures are maximum surface readings. B, H, and W are listed where "boiling," "hot," and "warm" are the only values available. P.P. 492 number is from U.S. Ceological Survey Professional Paper 492 (Waring, 1965). Circ. 790 number is from U.S. Ceological Survey Circular 790 (Muffler, 1979). NOAA number is on 1:250,000-scale overlays (see map 3 in pocket). Maps listed are standard U.S. Geologi-

cal Survey sheets. Parentheses in right column indicate site is not shown on the map by name or symbol.

\begin{tabular}{|c|c|c|c|}
\hline State & Lat. & Long. & Spring Name \\
\hline AK & 51.970 & 176.610 & HOT SPRINCS ON ADAK ISLAND \\
\hline AK & 51.925 & 177.160 & FUMAROLES ON KANAGA ISLAND \\
\hline AK & 51.810 & 177.790 & HOT SPRING ON TANAGA ISLAND \\
\hline AK & 51.775 & 178.793 & FUMAROLES ON GARELOI ISLAND \\
\hline AK & 51.762 & 178.770 & FUMAROLES ON GARELOI ISLAND \\
\hline AK & 51.967 & $178.444 \mathrm{E}$ & FUMAROLES ON LITTLE SITKIN ISLAND \\
\hline AK & 51.963 & 178.491E & FUMAROLES ON LITTLE SITKIN ISLAND \\
\hline AK & 51.944 & 178.547E & FUMAROLES ON LITTLE SITKIN ISLAND \\
\hline AK & 51.940 & $178.500 \mathrm{E}$ & HOT SPRING ON LITTLE SITKIN ISLAND \\
\hline AK & 52.960 & 169.710 & HOT SPRING ON KAGAMII ISLAND \\
\hline AK & 52.840 & 169.900 & CHUGINADAK HOT SPRINCS \\
\hline AK & 52.358 & 172.317 & HOT SPRING ON SECUAM ISLAND \\
\hline AK & 52.340 & 174.260 & HOT SPRINCS ON ATKA ISLAND \\
\hline AK & 52.270 & 174.042 & HOT SPRINGS ON ATKA ISLAND \\
\hline AK & 52.190 & 174.250 & HOT SPRINGS ON ATKA ISLAND \\
\hline AK & 52.042 & 176.108 & HOT SPRINGS ON GREAT SITKIN ISLAND \\
\hline AK & 52.870 & 173.130E & HOT SPRING ON ATTU ISLAND \\
\hline AK & 53.892 & 166.930 & MAKUSHIN VOLCANO FUMAROLES \\
\hline AK & 53.877 & 166.448 & HOT SPRINCS NEAR SUMMER BAY \\
\hline AK & 53.851 & 166.918 & HOT SPRINGS NEAR MAKUSHIN VOLCANO \\
\hline AK & 53.848 & 166.873 & HOT SPRINCS NEAR MAKUSHIN VOLCANO \\
\hline AK & 53.950 & 168.037 & HOT SPRINCS ON BOCOSLOF ISLAND \\
\hline AK & 53.443 & 168.092 & THERMAL SPRINCS IN OKMOK CALDERA \\
\hline AK & 53.253 & 168.358 & HOT SPRINGS NEAR HOT SPRINGS COVE \\
\hline AK & 53.242 & 168.365 & HOT SPRINGS NEAR HOT SPRINGS COVE \\
\hline AK & 53.233 & 168.300 & G ON UMNAK \\
\hline AK & 53.223 & 168.477 & HOT SPRINCS NEAR GEYSER BIGHT \\
\hline AK & 53.213 & 168.463 & HOT SPRINCS NEAR GEYSER BIGHT \\
\hline AK & 53.207 & 168.445 & HOT SPRINGS NEAR GEYSER BIGHT \\
\hline AK & 54.900 & 162.885 & HOT SPRING ON AMAGAT ISLAND \\
\hline
\end{tabular}

\begin{tabular}{|c|c|c|c|c|c|}
\hline \multicolumn{2}{|c|}{$\begin{array}{l}\text { Temperature } \\
{ }^{\circ} \mathrm{F}{ }^{\circ} \mathrm{C}\end{array}$} & $\begin{array}{l}\text { P.P. } \\
492\end{array}$ & $\begin{array}{c}\text { Circ. } \\
790\end{array}$ & NOAA & $1: 250,000($ AMS)map \\
\hline 154 & 68 & 33 & & 1 & ADAK \\
\hline 219 & 104 & 32 & & 1 & ADAK \\
\hline$H$ & $\mathrm{H}$ & 31 & & 2 & ADAK \\
\hline H & $\mathrm{H}$ & & & 1 & GARELOI ISLAND \\
\hline 144 & 62 & & & 2 & GARELOI ISLAND \\
\hline $\mathrm{H}$ & H & & & 1 & RAT ISLANDS \\
\hline $\mathrm{H}$ & $\mathrm{H}$ & & & 2 & RAT ISLANDS \\
\hline H & H & & & 3 & RAT ISLANDS \\
\hline $\mathrm{H}$ & H & 29 & & 4 & RAT ISLANDS \\
\hline H & H & 40 & & 1 & SAMALGA ISLAND \\
\hline H & H & 39 & & 2 & SAMALGA ISLAND \\
\hline H & H & 38 & & 1 & SECUAM \\
\hline $\mathrm{H}$ & H & 35 & & 1 & ATKA \\
\hline H & H & 36 & & 2 & ATKA \\
\hline 167 & 75 & 37 & & 3 & ATKA \\
\hline 210 & 99 & 34 & 16 & 1 & ADAK \\
\hline w & W & 28 & & 1 & ATTU \\
\hline 310 & 154 & 45 & & 1 & UNALASKA \\
\hline H & $\mathrm{H}$ & & & 2 & UNALASKA \\
\hline H & H & 45 & & 3 & UNALASKA \\
\hline 94 & 34 & 45 & & 4 & UNALASKA \\
\hline H & H & 44 & & 1 & UMNAK \\
\hline 212 & 100 & & & 2 & UMNAK \\
\hline 158 & 70 & 43 & 17 & 3 & UMNAK \\
\hline 192 & 89 & 43 & 17 & 4 & UMNAK \\
\hline 149 & 65 & 42 & 19 & 5 & UMNAK \\
\hline 214 & 101 & 41 & 18 & 6 & UMNAK \\
\hline 216 & 102 & 41 & 18 & 7 & UMNAK \\
\hline H & H & 41 & 18 & 8 & UMNAK \\
\hline H & H & 51 & & 1 & FALSE PASS \\
\hline
\end{tabular}

$1: 63360$ or $1: 62500(15$-minute) or $1: 24000$ (7.5-minute) quadrangle 


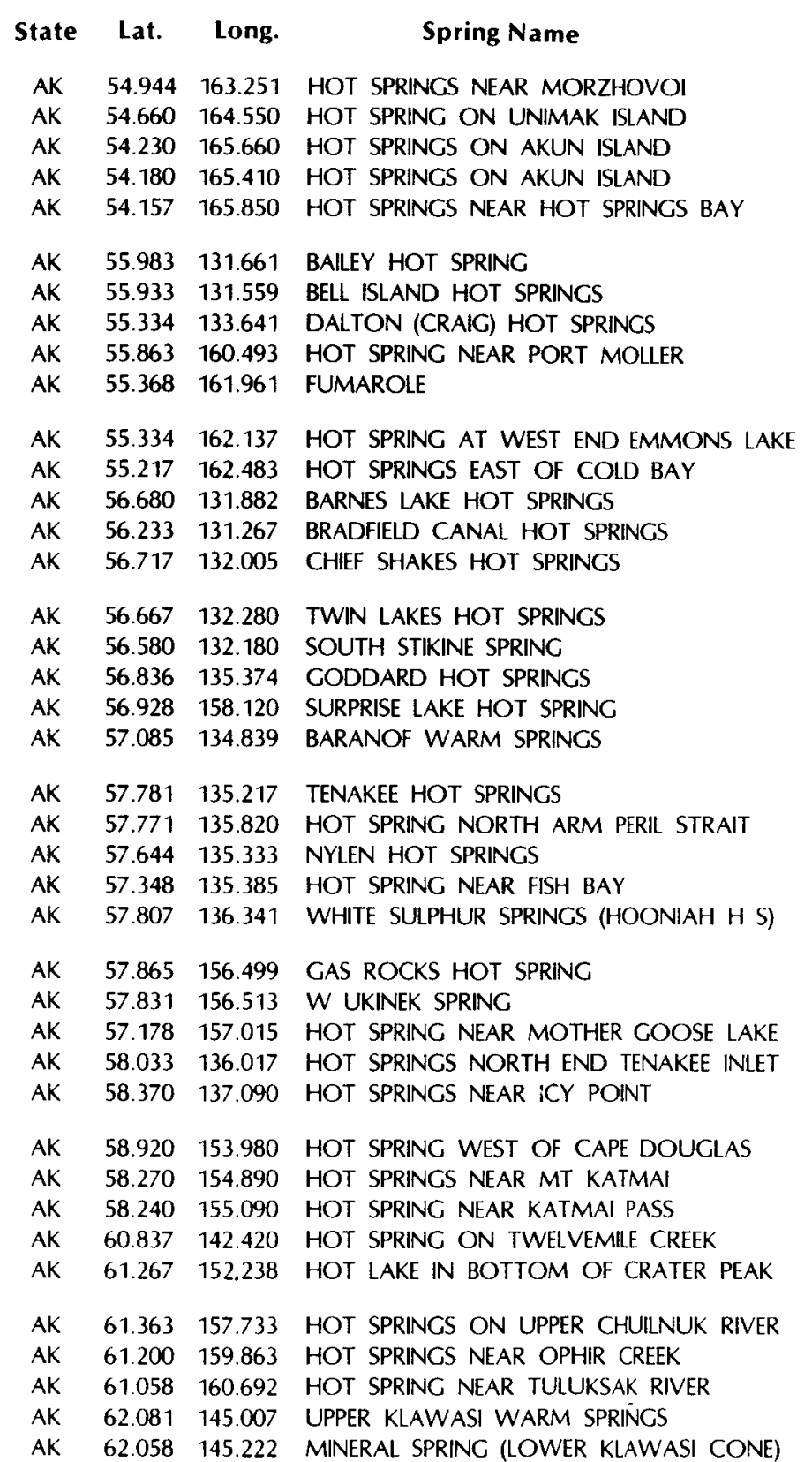

\begin{tabular}{|c|c|c|c|c|c|c|}
\hline \multicolumn{2}{|c|}{$\begin{array}{l}\text { Temperature } \\
{ }^{\circ} \mathrm{F} \\
{ }^{\circ} \mathrm{C}\end{array}$} & \multirow{2}{*}{$\begin{array}{r}\text { P.P. } \\
492 \\
50\end{array}$} & $\begin{array}{l}\text { Circ. } \\
790\end{array}$ & NOAA & $1: 250,000(A M S)$ map & $\begin{array}{l}1: 63360 \text { or } 1: 62500 \text { (15-minute) } \\
\text { or } 1: 24000(7.5 \text {-minute) quadrangle }\end{array}$ \\
\hline 145 & 63 & & & 1 & FALSE PASS & \\
\hline$H$ & $\mathrm{H}$ & 49 & & 1 & UNIMAK & \\
\hline $\mathrm{H}$ & $H$ & & & 1 & UNIMAK & \\
\hline H & $\mathrm{H}$ & & & 2 & UNIMAK & \\
\hline 181 & 83 & 46 & 20 & 3 & UNIMAK & \\
\hline 198 & 92 & 76 & 27 & 1 & KETCHIKAN & KETCHIKAN (D-5) 15 \\
\hline 165 & 74 & 79 & 28 & 2 & KETCHIKAN & KETCHIKAN (D-5) 15 \\
\hline 109 & 43 & & & 1 & CRAIG & CRAIG (B-5) 15 \\
\hline 160 & 71 & 54 & & 1 & PORT MOLLR & PORT MOLLER (D-2) 15 \\
\hline $\mathrm{H}$ & $\mathrm{H}$ & 52 & & 1 & PORT MOLLER & \\
\hline 68 & 20 & & & 1 & COLD BAY & $:$ \\
\hline 129 & 54 & & 21 & 2 & COLD BAY & \\
\hline 79 & 26 & & & 1 & BRADFIELD CANAL & (BRADFIELD CANAL (C-6) 15) \\
\hline 135 & 57 & & & 2 & BRADFIELD CANAL & (BRADFIELD CANAL (A-4) 15) \\
\hline 122 & 50 & 73 & 26 & 1 & PETERSBURG & (PETERSBURG (C-1) 15) \\
\hline 70 & 21 & & & 2 & PETERSBURG & (PETERSBURG (C-1) 15) \\
\hline w & W & 74 & & 3 & PETERSBURG & (PETERSBURC (C-1) 15) \\
\hline 153 & 67 & 70 & 25 & 1 & PORT ALEXANDER & PORT ALEXANDER (D-5) 15 \\
\hline 73 & 23 & & & 1 & CHIGNIK & (CHIGNIK (D-1) 15) \\
\hline 124 & 51 & 69 & & 1 & SITKA & SITKA (A-3) 15 \\
\hline 109 & 43 & 67 & & 1 & SITKA & SITKA (D-4) 15 \\
\hline 100 & 38 & 66 & & 2 & SITKA & (SITKA (D-6) 15) \\
\hline 120 & 49 & & & 3 & SITKA & (SITKA (C-5) 15) \\
\hline 117 & 47 & 68 & 24 & 4 & SITKA & SITKA (B-5) 15 \\
\hline 111 & 44 & 65 & 23 & 1 & SITKA & SITKA (D-8) 15 \\
\hline 127 & 53 & 56 & & 1 & UGASHIK & (UGASHIK (D-2) 15) \\
\hline 178 & 81 & & & 2 & UGASHIK & (UGASHIK (D-2) 15) \\
\hline 138 & 59 & & & 1 & UGASHIK & (UGASHIK (A-4) 15) \\
\hline 180 & 82 & & 22 & 1 & MT. FAIRWEATHER & (MT. FAIRWEATHER (A-1) 15) \\
\hline 153 & 67 & & & 1 & MT. FAIRWEATHER & (MT. FAIRWEATHER (B-4) 15) \\
\hline $\mathrm{H}$ & H & 59 & & 1 & AFOGNAK & (AFOGNAK (D-6) 15) \\
\hline $\mathrm{H}$ & $\mathrm{H}$ & 58 & & 1 & MT. KATMAI & (MT. KATMAI (B-3) 15) \\
\hline $\mathrm{H}$ & $H$ & 57 & & 1 & MT. KATMAI & (MT. KATMAI (A-4) 15) \\
\hline w & $w$ & 27 & & 1 & BERING GLACIER & \\
\hline $\mathrm{H}$ & $H$ & & & 1 & TYONEK & (TYONEK (B-6) 15) \\
\hline $\mathrm{H}$ & $\mathrm{H}$ & & & 1 & SLEETMUTE & (SLEETMUTE (B-4) 15) \\
\hline 145 & 63 & 26 & & 1 & RUSSIAN MISSION & (RUSSIAN MISSIÓN (A-2) 15) \\
\hline$H$ & $\mathrm{H}$ & 25 & & 1 & RUSSIAN MISSION & (RUSSIAN MISSION (A-5) 15) \\
\hline 86 & 30 & & & 1 & GULKANA & GUIKANA (A-3) 15 \\
\hline 82 & 28 & & & 2 & GULKANA & GULKANA (A-3) 15 \\
\hline
\end{tabular}




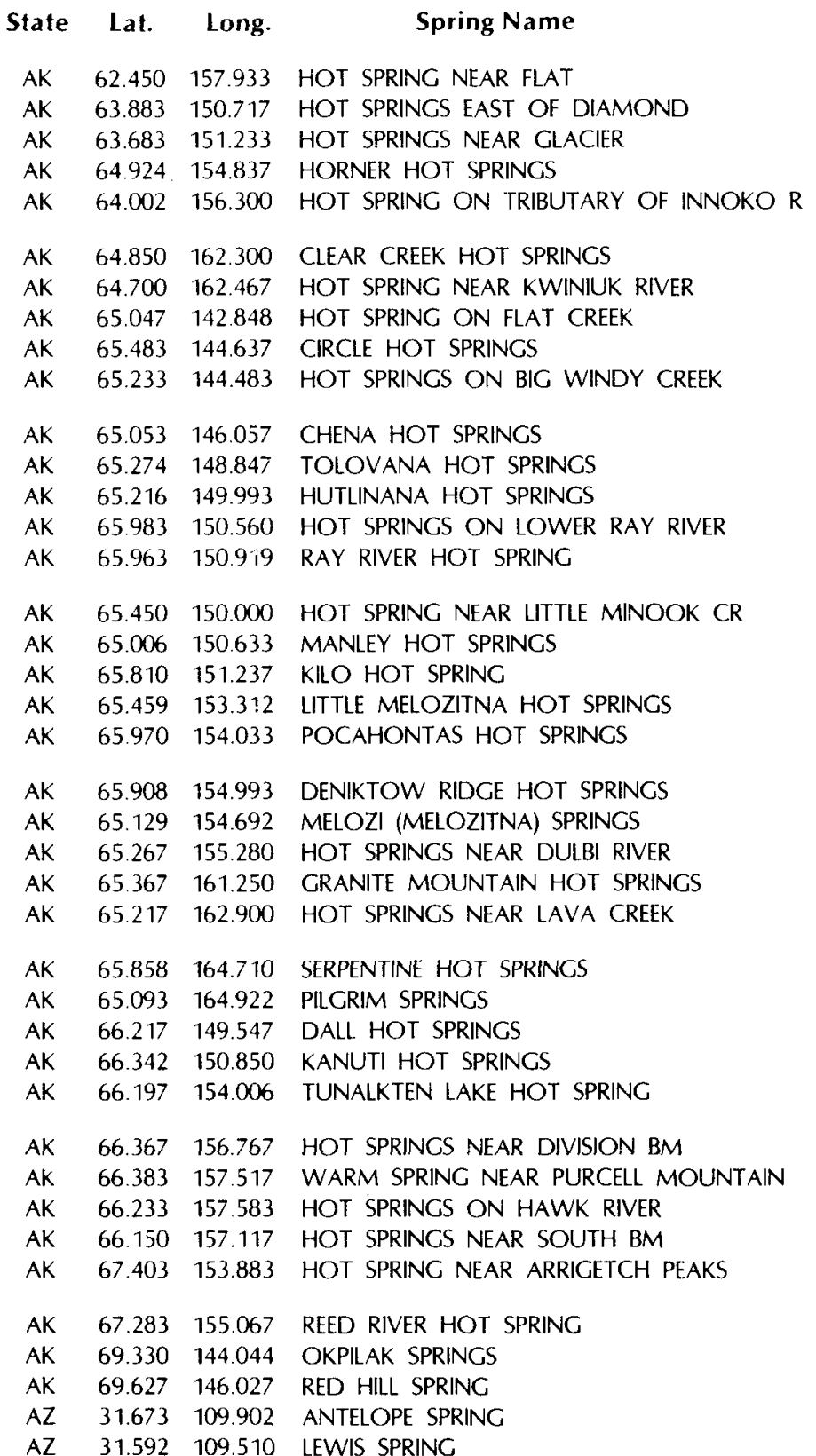

\begin{tabular}{|c|c|c|c|c|c|c|}
\hline \multicolumn{2}{|c|}{$\begin{array}{l}\text { Temperature } \\
{ }^{\circ} \mathrm{F} \quad{ }^{\circ} \mathrm{C}\end{array}$} & \multirow{2}{*}{$\begin{array}{r}\text { P.P. } \\
492 \\
24\end{array}$} & $\begin{array}{l}\text { Circ. } \\
790\end{array}$ & NOAA & $1: 250,000$ (AMS)map & $\begin{array}{l}1: 63360 \text { or } 1: 62500(15 \text {-minute }) \\
\text { or } 1: 24000(7.5 \text {-minute }) \text { quadrangle }\end{array}$ \\
\hline W & W & & & 1 & IDITAROD & (IDITAROD (B-4) 15) \\
\hline W & W & 22 & & 1 & MT. MCKINLEY & (MT. MCKINLEY (D-2) 15) \\
\hline W & W & 23 & & 1 & MT. MCKINLEY & (MT. MCKINLEY (C-3) 15) \\
\hline 117 & 47 & 9 & & 1 & RUBY & RUBY (D-4) 15 \\
\hline $\mathrm{H}$ & $H$ & 13 & & 1 & NULATO & (NULATO (A-1) 15) \\
\hline 149 & 65 & & 5 & 1 & SOLOMON & (SOLOMON (D-1) 15) \\
\hline 106 & 41 & 7 & & 2 & SOLOMON & (SOLOMON (C-1) 15) \\
\hline W & W & 21 & & 1 & CHARLEY RIVER & (CHARLEY RIVER (A-4) 15) \\
\hline 135 & 57 & 19 & 15 & 1 & CIRCLE & CIRCLE (B-2) 15 \\
\hline $\mathrm{H}$ & $\mathrm{H}$ & 20 & & 2 & CIRCLE & (CIRCLE (A-1) 15) \\
\hline 153 & 67 & 18 & 14 & 1 & CIRCLE & CIRCLE (A-5) 15 \\
\hline 140 & 60 & 17 & 12 & 1 & LIVENGOOD & LIVENGOOD (B-4) 15 \\
\hline 109 & 43 & 15 & & 1 & LIVENGOOD & LIVENGOOD (A-6) 15 \\
\hline 142 & 61 & & & 1 & TANANA & (TANANA (D-2) 15) \\
\hline 117 & 47 & & & 2 & TANANA & TANANA (D-2) 15 \\
\hline $\mathrm{H}$ & $\mathrm{H}$ & 16 & & 3 & TANANA & (TANANA (B-1) 15) \\
\hline 133 & 56 & 14 & 13 & 4 & TANANA & TANANA (A-2) \\
\hline 122 & 50 & & & 1 & TANANA & TANANA (D-3) \\
\hline 176 & 80 & 11 & 9 & 1 & MELOZITNA & MELOZITNA (B-1) 15 \\
\hline $\mathrm{H}$ & $\mathrm{H}$ & & & 1 & MELOZITNA & MELOZITNA (D-3) 15 \\
\hline $\mathrm{H}$ & $\mathrm{H}$ & & & 2 & MELOZITNA & MELOZITNA (D-4) 15 \\
\hline 131 & 55 & 10 & 8 & 3 & MELOZITNA & MELOZITNA (A-4) 15 \\
\hline 126 & 52 & & 7 & 1 & MELOZITNA & (MELOZITNA (B-5) 15) \\
\hline 120 & 49 & 8 & & 1 & CANDLE & (CANDLE (B-5) 15) \\
\hline 127 & 53 & & 4 & 1 & BENDELEBEN & (BENDELEBEN (A-2) 15) \\
\hline 171 & 77 & 4 & 2 & 1 & BENDELEBEN & BENDELEBEN (D-6) 15 \\
\hline 178 & 81 & 6 & 3 & 2 & BENDELEBEN & BENDELEBEN (A-6) 15 \\
\hline 129 & 54 & & & 1 & BEAVER & (BEAVER (A-6) 15) \\
\hline 151 & 66 & & 11 & 1 & BETTLES & (BETTLES (B-2) 15) \\
\hline & & & & 1 & HUGHES & HUCHES (A-3) 15 \\
\hline 140 & 60 & 3 & & 1 & SHUNGNAK & \\
\hline 68 & 20 & & & 1 & SHUNGNAK & \\
\hline 122 & 50 & & & 2 & SHUNGNAK & \\
\hline 122 & 50 & & 6 & 3 & SHUNGNAK & \\
\hline w & W & & & 1 & SURVEY PASS & \\
\hline 122 & 50 & 1 & 10 & 1 & SURVEY PASS & \\
\hline 119 & 49 & & & 1 & MT. MICHELSON & (MT. MICHELSON (B-1) 15) \\
\hline 91 & 33 & & & 1 & MT. MICHELSON & MT. MICHELSON (C-4) 15 \\
\hline 79 & 26 & & & 1 & DOUGLAS & HAY MOUNTAIN 7.5 \\
\hline 68 & 20 & & & 2 & DOUGLAS & (SWISSHELM MTN. 15) \\
\hline
\end{tabular}




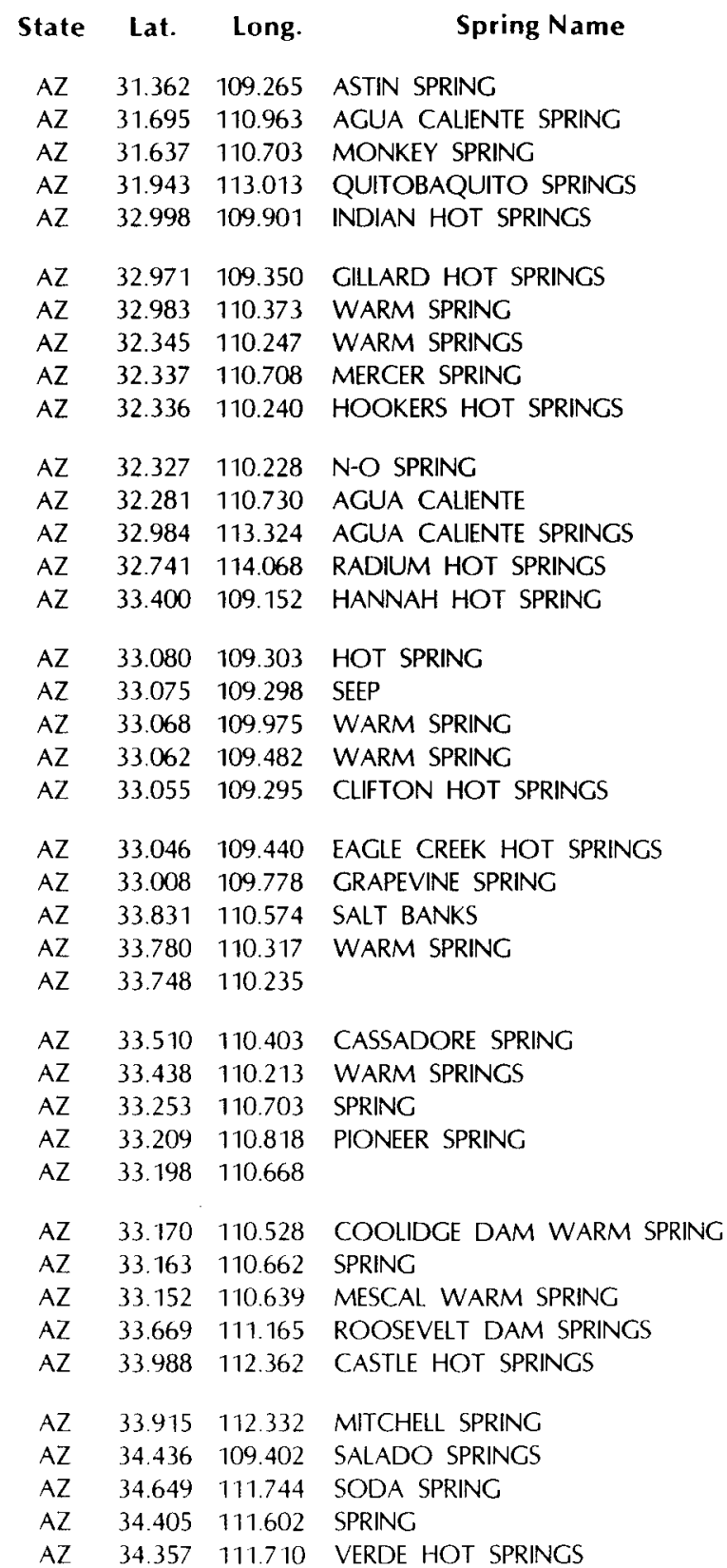

\begin{tabular}{|c|c|c|c|c|c|c|}
\hline \multicolumn{2}{|c|}{$\begin{array}{l}\text { Temperature } \\
{ }^{\circ} \mathrm{F} \quad{ }^{\circ} \mathrm{C}\end{array}$} & \multirow[t]{2}{*}{$\begin{array}{l}\text { P.P. } \\
492\end{array}$} & $\begin{array}{l}\text { Circ. } \\
790\end{array}$ & NOAA & 1:250,000(AMS)map & $\begin{array}{l}1: 63360 \text { or } 1: 62500(15 \text {-minute }) \\
\text { or } 1: 24000(7.5 \text {-minute }) \text { quadrangle }\end{array}$ \\
\hline 75 & 24 & & & 3 & DOUGLAS & COLLEGE PEAKS 15 \\
\hline 90 & 32 & 20 & & 1 & NOGALES & MOUNT WRIGHTSON 15 \\
\hline 82 & 28 & & & 2 & NOGALES & SONOITA 7.5 \\
\hline 90 & 32 & 18 & & 1 & LUKEVILLE & QUITOBAQUITO SPRINCS 15 \\
\hline 118 & 48 & 14 & & 1 & SILVER CITY & THATCHER 15 \\
\hline 180 & 82 & & 32 & 2 & SILVER CITY & GUTHRIE 15 \\
\hline 79 & 26 & 12 & & 1 & TUCSON & COBRE GRANDE MTN. 7.5 \\
\hline 91 & 33 & & & 2 & TUCSON & (WINCHESTER MTS. 15) \\
\hline 77 & 25 & & & 3 & TUCSON & BELLOTA RANCH 15 \\
\hline 127 & 53 & 19 & & 4 & TUCSON & WINCHESTER MTS. 15 \\
\hline 73 & 23 & & & 5 & TUCSON & WINCHESTER MTS. 15 \\
\hline 86 & 30 & & & 6 & TUCSON & BELLOTA RANCH 15 \\
\hline 102 & 39 & 11 & & 1 & $\mathrm{AJO}$ & AGUA CALIENTE 7.5 \\
\hline 140 & 60 & & & 1 & EL CENTRO & WELLTON MESA 7.5 \\
\hline 133 & 56 & & & 1 & CLIFTON & DUTCH BLUE CREEK 7.5 \\
\hline 138 & 59 & & 31 & 2 & CUIFTON & CLIFTON 15 \\
\hline 95 & 35 & & 31 & 3 & CLIFTON & (CLIFTON 15) \\
\hline 90 & 32 & 13 & & 4 & CLIFTON & FORT THOMAS 15 \\
\hline 73 & 23 & & & 5 & CLIFTON & (CLIFTON 15) \\
\hline 102 & 39 & 17 & & 6 & CLIFTON & (CLIFTON 15) \\
\hline 97 & 36 & 16 & 30 & 7 & CLIFTON & CUIFTON 15 \\
\hline 91 & 33 & & & 8 & CLIFTON & (FORT THOMAS 15) \\
\hline 78 & 26 & 9 & & 1 & MESA & (BLUE HOUSE MTN. 15) \\
\hline W & w & & & 2 & MESA & CARRIZO SE 7.5 \\
\hline 83 & 28 & & & 3 & MESA & (FORKS BUTTE 7.5) \\
\hline 73 & 23 & & & 4 & MESA & CASSADORE SPRING 7.5 \\
\hline 86 & 30 & & & 5 & MESA & BRONCO GULCH 7.5 \\
\hline 75 & 24 & & & 6 & MESA & (CUTTER 7.5) \\
\hline 91 & 33 & & & 7 & MESA & (EL CAPITAN MTN. 7.5 ) \\
\hline 99 & 37 & & & 8 & MESA & (MESCAL WARM SPRING 7.5) \\
\hline 99 & 37 & & & 9 & MESA & COOLIDGE DAM 7.5 \\
\hline 82 & 28 & & & 10 & MESA & MESCAL WARM SPRING 7.5 \\
\hline 84 & 29 & & & 11 & MESA & MESCAL WARM SPRING 7.5 \\
\hline 118 & 48 & & & 1 & MESA & THEODORE ROOSEVELT DAM 7.5 \\
\hline 115 & 46 & 8 & & 1 & PHOENIX & GOVERNORS PEAK 7.5 \\
\hline 79 & 26 & & & 2 & PHOENIX & GOVERNORS PEAK 7.5 \\
\hline 72 & 22 & 7 & & 1 & SAINT JOHNS & SALADO 7.5 \\
\hline 75 & 24 & 5 & & 1 & HOLBROOK & CASNER BUTTE 7.5 \\
\hline 72 & 22 & & & 2 & HOLBROOK & STRAWBERRY 7.5 \\
\hline 104 & 40 & 6 & & 3 & HOLBROOK & VERDE HOT SPRINCS 7.5 \\
\hline
\end{tabular}




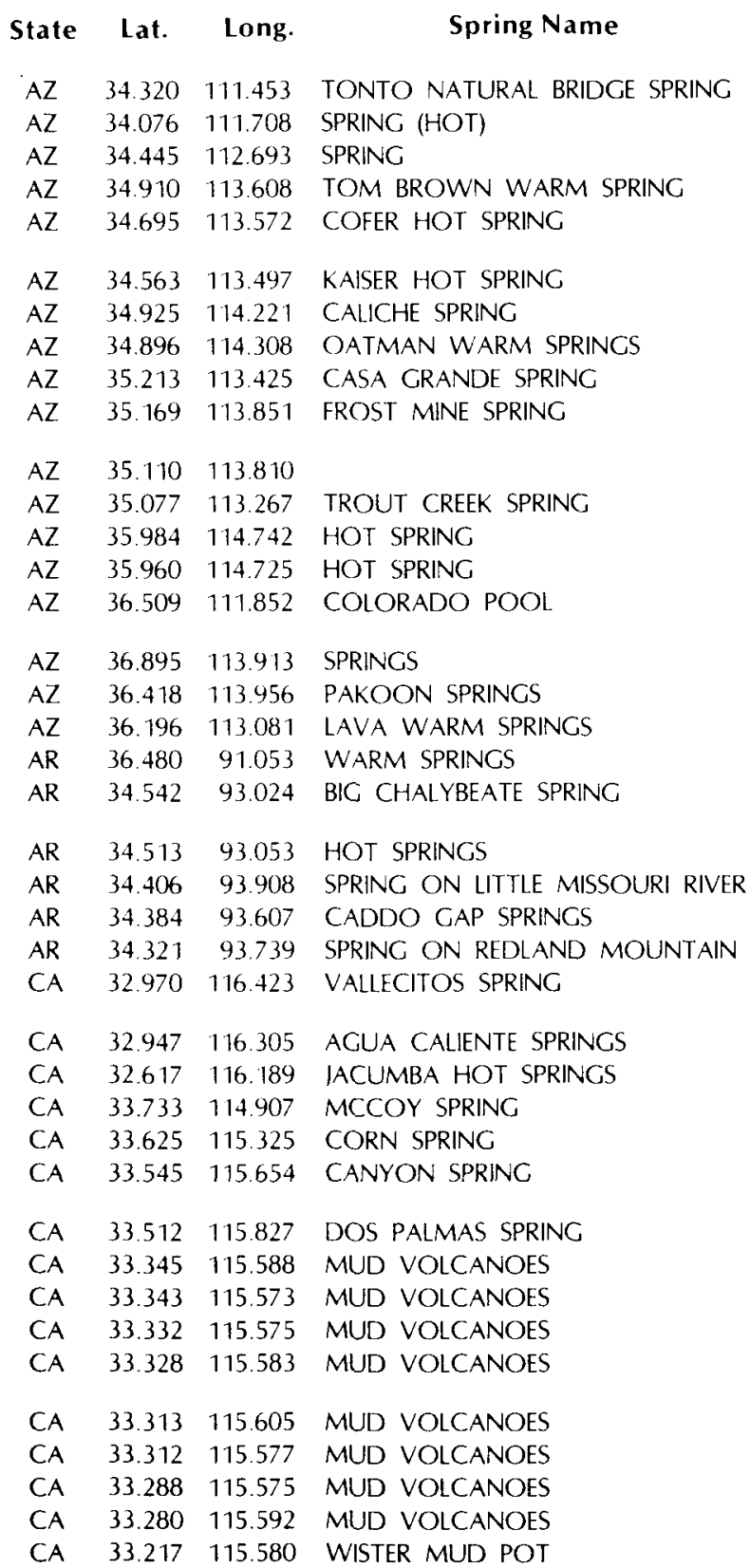

\begin{tabular}{|c|c|}
\hline$e_{\mathrm{o}}$ & \\
\hline 72 & 22 \\
\hline 99 & 37 \\
\hline 72 & 22 \\
\hline 82 & 28 \\
\hline 97 & 36 \\
\hline 99 & 37 \\
\hline 79 & 26 \\
\hline 91 & 33 \\
\hline 72 & 22 \\
\hline 70 & 21 \\
\hline 73 & 23 \\
\hline 81 & 27 \\
\hline $\mathrm{H}$ & $\mathrm{H}$ \\
\hline 86 & 30 \\
\hline 86 & 30 \\
\hline 79 & 26 \\
\hline 86 & 30 \\
\hline 90 & 32 \\
\hline 82 & 28 \\
\hline 79 & 26 \\
\hline 147 & 64 \\
\hline 74 & 23 \\
\hline 95 & 35 \\
\hline 77 & 25 \\
\hline 79 & 26 \\
\hline 101 & 38 \\
\hline 101 & 38 \\
\hline 82 & 28 \\
\hline 72 & 22 \\
\hline 97 & 36 \\
\hline 84 & 29 \\
\hline$W$ & W \\
\hline$W$ & W \\
\hline$W$ & W \\
\hline$w$ & 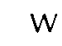 \\
\hline$W$ & W \\
\hline W & 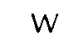 \\
\hline$W$ & $\mathrm{v}$ \\
\hline$W$ & 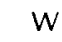 \\
\hline 100 & 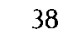 \\
\hline
\end{tabular}

P.P. Circ. NOAA 1:250,000(AMS)map 492790

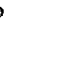

$\begin{array}{ll}4 & \text { HOLBROOK } \\ 5 & \text { HOLBROOK } \\ 1 & \text { PRESCOTT } \\ 1 & \text { PRESCOTT } \\ 2 & \text { PRESCOTT }\end{array}$

PRESCOTT

NEEDLES

NEEDLES

WILLIAMS

WILLIAMS

WILLIAMS

WILLIAMS

KINGMAN

KINGMAN

MARBLE CANYON

GRAND CANYON

GRAND CANYON

GRAND CANYON

POPLAR BLUFF

LITTLE ROCK

LITTLE ROCK

LITTLE ROCK

LITTLE ROCK

IITTLE ROCK

SAN DIECO

SAN DIEGO

SAN DIEGO

SALTON SEA

SALTON SEA

SALTON SEA

SALTON SEA

SALTON SEA

SALTON SEA

SALTON SEA

SALTON SEA

SALTON SEA

SALTON SEA

SALTON SEA

SALTON SEA

6412 SALTON SEA
1:63360 or 1:62500 (15-minute)

or 1:24000 (7.5-minute) quadrangle

BUCKHEAD MESA 7.5

CHALK MOUNTAIN 7.5

KIRKLAND 7.5

TOM BROWN CANYON 7.5

WIKIEUP 7.5

YUCCA NW 7.5

WARM SPRINGS 7.5

(HUALAPAI PEAK NE 7.5)

(DEAN PEAK 7.5)

RINGBOLT RAPIDS 7.5

RINGBOLT RAPIDS 7.5

(EMMETT WASH 15)

LITTLEFIELD 15

PAKOON SPRINCS 7.5

VULCANS THRONE 7.5

WARM SPRINGS 7.5

HOT SPRINGS NORTH 7.5

HOT SPRINGS NORTH 7.5

(ATHENS 15)

(GLENWOOD 15)

(GLENWOOD 15)

MONUMENT PEAK 7.5

ACUA CALIENTE 7.5

(JACUMBA 7.5)

MCCOY SPRING 15

CHUCKWALLA MOUNTAINS 15

HAYFIELD 15

OROCOPIA CANYON 7.5

(WISTER 7.5)

(WISTER 7.5)

(WISTER 7.5)

(WISTER 7.5)

(WISTER 7.5)

(WISTER 7.5)

(WISTER 7.5)

(WISTER 7.5)

NILAND 7.5 


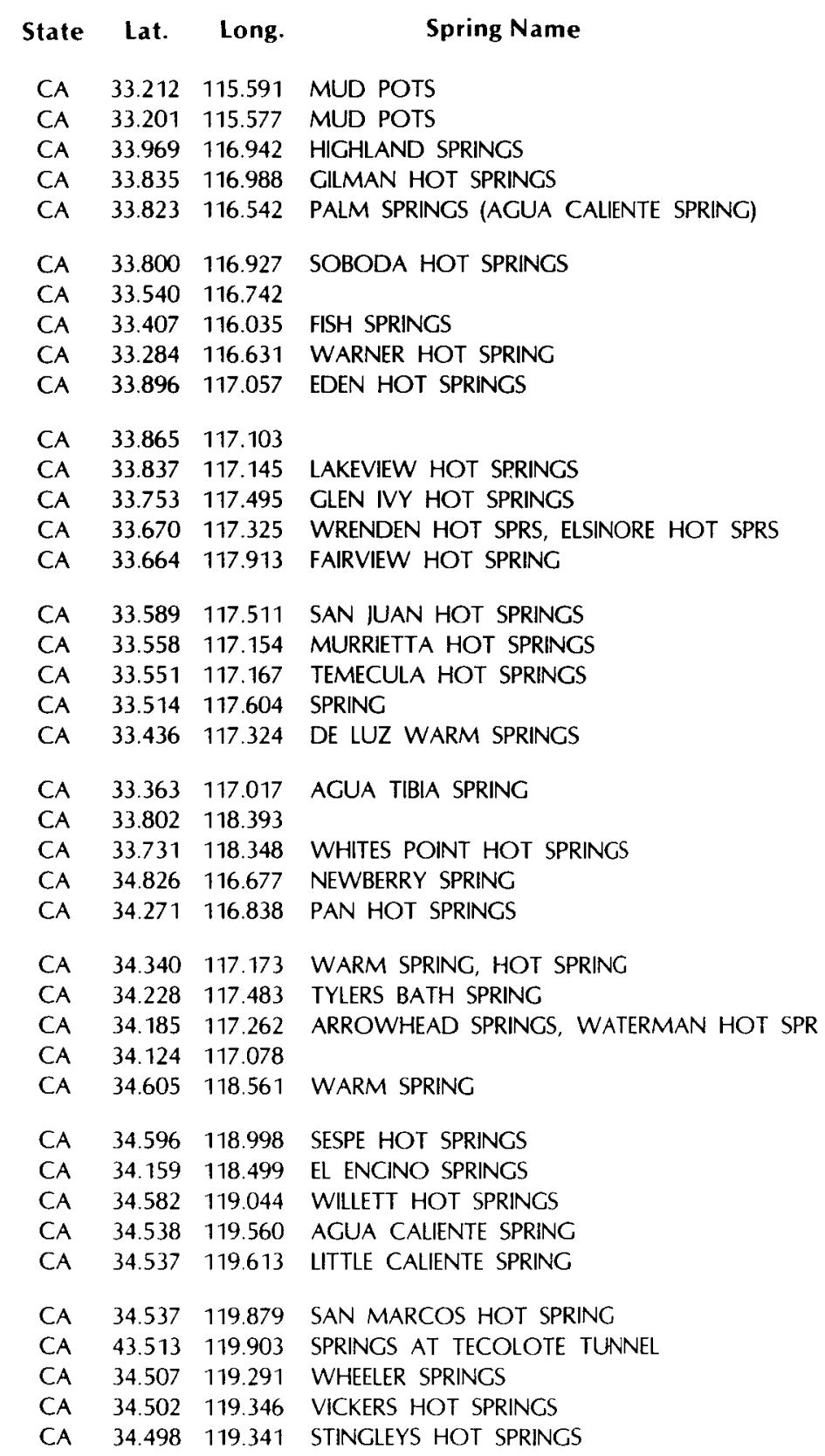

\begin{tabular}{|c|c|c|c|c|c|c|}
\hline \multicolumn{2}{|c|}{ Temperature } & $\begin{array}{l}\text { P.P. } \\
492\end{array}$ & $\begin{array}{l}\text { Circ. } \\
790\end{array}$ & NOAA & $1: 250,000(A M S)$ map & $\begin{array}{l}1: 63360 \text { or } 1: 62500(15 \text {-minute }) \\
\text { or } 1: 24000(7.5 \text {-minute) quadrangle }\end{array}$ \\
\hline 100 & 38 & & 64 & 13 & SALTON SEA & NILAND 7.5 \\
\hline 100 & 38 & & 64 & 14 & SALTON SEA & NILAND 7.5 \\
\hline 112 & 44 & $172 \mathrm{~A}$ & & 1 & SANTA ANA & BEAUMONT 7.5 \\
\hline 117 & 47 & 173 & & 2 & SANTA ANA & SAN IACINTO 7.5 \\
\hline 104 & 40 & 175 & & 3 & SANTA ANA & PALM SPRINGS 7.5 \\
\hline 102 & 39 & 174 & & 4 & SANTA ANA & SAN IACINTO 7.5 \\
\hline w & w & & & 5 & SANTA ANA & (IDYLLWILD 15) \\
\hline 90 & 32 & 182 & & 6 & SANTA ANA & (OASIS 7.5) \\
\hline 138 & 59 & 179 & & 7 & SANTA ANA & WARNER SPRINCS 7.5 \\
\hline 109 & 43 & 172 & & 1 & SANTA ANA & EL CASCO 7.5 \\
\hline W & w & & & 2 & SANTA ANA & (LAKEVIEW 7.5) \\
\hline 100 & 38 & 171 & & 3 & SANTA ANA & PERRIS 7.5 \\
\hline 131 & 55 & 167 & & 4 & SANTA ANA & LAKE MATTHEWS 7.5 \\
\hline 125 & 52 & 168 & & 5 & SANTA ANA & (ELSINORE 7.5) \\
\hline 96 & 36 & 165 & & 6 & SANTA ANA & (NEWPORT BEACH 7.5) \\
\hline 120 & 49 & 166 & & 7 & SANTA ANA & CANADA GOBERNADORA 7.5 \\
\hline 132 & 56 & 170 & & 8 & SANTA ANA & MURRIETTA 7.5 \\
\hline 116 & 47 & & & 9 & SANTA ANA & MURRIETTA 7.5 \\
\hline 95 & 35 & & & 10 & SANTA ANA & CANADA GOBERNADORA 7.5 \\
\hline 85 & 29 & 177 & & 11 & SANTA ANA & (FALLBROOK 7.5 ) \\
\hline 92 & 33 & 178 & & 12 & SANTA ANA & (PALA 7.5) \\
\hline 77 & 25 & & & 1 & LONG BEACH & (REDONDO BEACH 7.5) \\
\hline 115 & 46 & & & 2 & LONG BEACH & (SAN PEDRO 7.5) \\
\hline 77 & 25 & 157 & & 1 & SAN BERNARDINO & NEWBERRY 15 \\
\hline 88 & 31 & 164 & & 2 & SAN BERNARDINO & (BIG BEAR CITY 7.5) \\
\hline 108 & 42 & 160 & & 1 & SAN BERNARDINO & LAKE ARROWHEAD 7.5 \\
\hline 92 & 33 & 158 & & 2 & SAN BERNARDINO & (DEVORE 7.5) \\
\hline 194 & 90 & 162 & 62 & 3 & SAN BERNARDINO & SAN BERNARDINO NORTH 7.5 \\
\hline 90 & 32 & 163 & & 4 & SAN BERNARDINO & (YUCAIPA 7.5 ) \\
\hline 92 & 33 & 112 & & 1 & LOS ANCELES & WARM SPRINCS MOUNTAIN 7.5 \\
\hline 194 & 90 & 111 & 61 & 2 & LOS ANGELES & DEVILS HEART PEAK 7.5 \\
\hline 79 & 26 & & & 3 & LOS ANGELES & VAN NUYS 7.5 \\
\hline 108 & 42 & 110 & & 1 & LOS ANGELES & (TOPATOPA MOUNTAINS 7.5) \\
\hline 133 & 56 & & & 2 & LOS ANGELES & HILDRETH PEAK 7.5 \\
\hline 90 & 32 & 104 & & 3 & LOS ANGELES & HILDRETH PEAK 7.5 \\
\hline 110 & 43 & 102 & & 4 & LOS ANGELES & LAKE CACHUMA 7.5 \\
\hline 93 & 34 & & & 5 & LOS ANGELES & (LAKE CACHUMA 7.5) \\
\hline 102 & 39 & 109 & & 6 & LOS ANGELES & WHEELER SPRINGS 7.5 \\
\hline 124 & 51 & 106 & & 7 & LOS ANGELES & (WHEELER SPRINCS 7.5) \\
\hline 123 & 51 & 107 & & 8 & LOS ANGELES & MATILIJA 7.5 \\
\hline
\end{tabular}




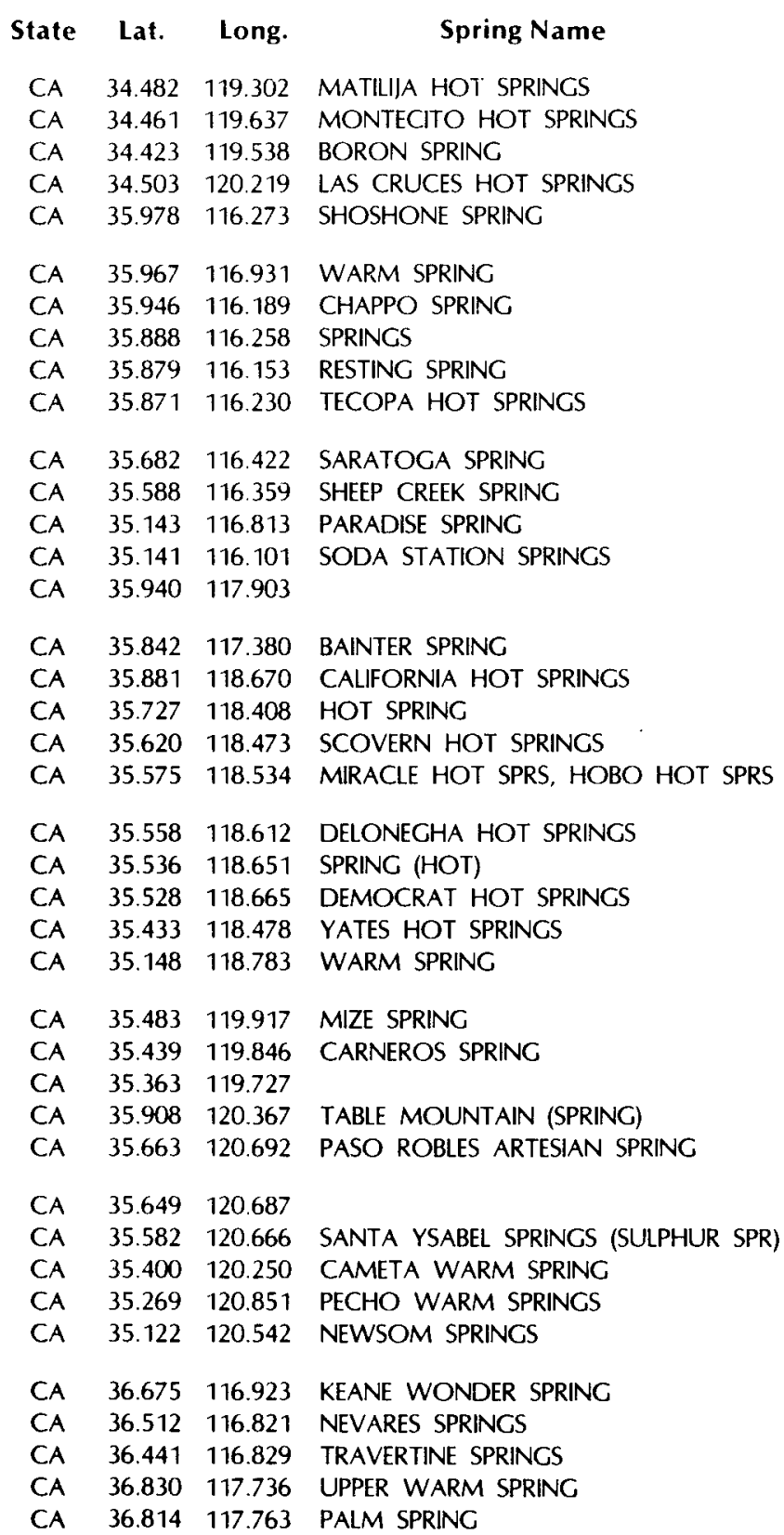

\begin{tabular}{|c|c|c|c|c|c|c|}
\hline \multicolumn{2}{|c|}{$\begin{array}{l}\text { Temperature } \\
{ }^{\circ} \mathrm{F} \quad{ }^{\circ} \mathrm{C}\end{array}$} & \multirow{2}{*}{$\begin{array}{r}\text { P.P. } \\
492 \\
108\end{array}$} & $\begin{array}{l}\text { Circ. } \\
790\end{array}$ & \multicolumn{2}{|c|}{ NOAA 1:250,000(AMS)map } & $\begin{array}{l}1: 63360 \text { or } 1: 62500(15 \text {-minute }) \\
\text { or } 1: 24000(7.5 \text {-minute) quadrangle }\end{array}$ \\
\hline 109 & 43 & & & 9 & LOS ANGELES & MATILIIA 7.5 \\
\hline 118 & 48 & 103 & & 10 & LOS ANGELES & SANTA BARBARA 7.5 \\
\hline 72 & 22 & & & 11 & LOS ANGELES & (CARPINTERIA 7.5) \\
\hline 99 & 37 & 101 & & 1 & SANTA MARIA & SOLVANG 7.5 \\
\hline 90 & 32 & & & 1 & TRONA & SHOSHONE 15 \\
\hline W & W & & & 2 & TRONA & WINGATE WASH 15 \\
\hline 80 & 27 & 145 & & 3 & TRONA & TECOPA 15 \\
\hline w & W & & & 4 & TRONA & SHOSHONE 15 \\
\hline 80 & 27 & 147 & & 5 & TRONA & TECOPA 15 \\
\hline 118 & 48 & 146 & 59 & 6 & TRONA & TECOPA 15 \\
\hline 82 & 28 & 154 & & 7 & TRONA & AVAWATZ PASS 15 \\
\hline 73 & 23 & & & 8 & TRONA & AVAWATZ PASS 15 \\
\hline 100 & 38 & 155 & & 9 & TRONA & LANE MOUNTAIN 15 \\
\hline 75 & 24 & 156 & & 10 & TRONA & SODA LAKE 15 \\
\hline 80 & 27 & 143 & & 1 & TRONA & (LITTLE LAKE 15) \\
\hline 92 & 33 & & & 2 & TRONA & TRONA 15 \\
\hline 122 & 50 & 137 & & 1 & BAKERSFIELD & CALIFORNIA HOT SPRINGS 15 \\
\hline 113 & 45 & 148 & & 2 & BAKERSFIELD & LAKE ISABELLA NORTH 7.5 \\
\hline 133 & 56 & 149 & 60 & 3 & BAKERSFIELD & LAKE ISABELLA SOUTH 7.5 \\
\hline 119 & 48 & 150 & & 4 & BAKERSFIELD & MIRACLE HOT SPRINCS 7.5 \\
\hline 112 & 44 & 151 & & 5 & BAKERSFIELD & MIRACIE HOT SPRINGS 7.5 \\
\hline w & W & & & 6 & BAKERSFIELD & DEMOCRAT HOT SPRINGS 7.5 \\
\hline 115 & 46 & 152 & & 7 & BAKERSFIELD & DEMOCRAT HOT SPRINGS 7.5 \\
\hline 100 & 38 & 153 & & 8 & BAKERSFIELD & PIUTE PEAK 7.5 \\
\hline$w$ & W & & & 9 & BAKERSFIELD & ARVIN 7.5 \\
\hline 73 & 23 & & & 1 & BAKERSFIELD & (LAS YEGUAS RANCH 7.5) \\
\hline 90 & 32 & & & 2 & BAKERSFIELD & CARNEROS ROCKS 7.5 \\
\hline 93 & 34 & & & 3 & BAKERSFIELD & (REWARD 7.5) \\
\hline 88 & 31 & & & 1 & SAN LUIS OBISPO & (THE DARK HOLE 7.5) \\
\hline 102 & 39 & & & 2 & SAN LUIS OBISPO & (PASO ROBLES 7.5) \\
\hline 108 & 42 & & & 3 & SAN LUIS OBISPO & (PASO ROBLES 7.5) \\
\hline 92 & 33 & 97 & & 4 & SAN LUIS OBISPO & TEMPLETON 7.5 \\
\hline 73 & 23 & & & 5 & SAN LUIS OBISPO & (LA PANZA RANCH 7.5) \\
\hline 95 & 35 & 99 & & 6 & SAN LUIS OBISPO & (MORRO BAY SOUTH 7.5) \\
\hline 99 & 37 & 100 & & 7 & SAN LUIS OBISPO & OCEANO 7.5 \\
\hline 93 & 34 & $140 \mathrm{~A}$ & & 1 & DEATH VALLEY & CHLORIDE CLIFF 15 \\
\hline 102 & 39 & & & 2 & DEATH VALLEY & CHLORIDE CLIFF 15 \\
\hline 90 & 32 & & & 3 & DEATH VALLEY & FURNACE CREEK 15 \\
\hline w & w & & & 1 & DEATH VALLEY & DRY MOUNTAIN 15 \\
\hline 0 & 49 & & & 2 & DEATH VALLEY & WAUCORA IWAGH \\
\hline
\end{tabular}




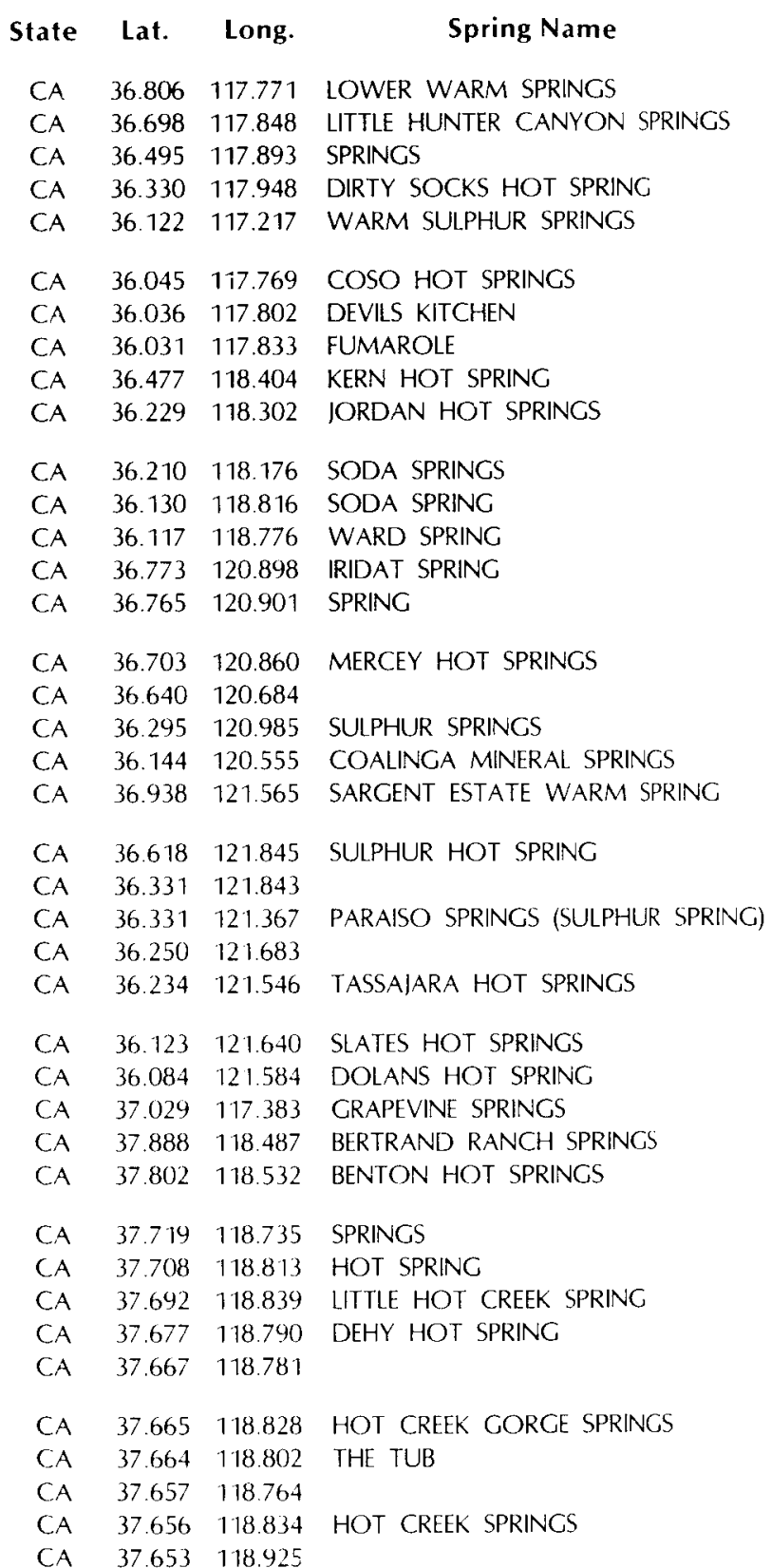

\begin{tabular}{|c|c|c|c|c|c|}
\hline \multicolumn{2}{|c|}{ Temperature } & P.P. & Circ. & NOAA & $1: 250,000$ (AMS)map \\
\hline${ }^{\circ} \mathbf{F}$ & C & 492 & 790 & & \\
\hline 110 & 43 & 139 & & 3 & DEATH VALLEY \\
\hline 81 & 27 & & & 4 & DEATH VALLEY \\
\hline 86 & 30 & & & 5 & DEATH VALLEY \\
\hline 93 & 34 & & & 6 & DEATH VALLEY \\
\hline 80 & 27 & 144 & & 7 & DEATH VALLEY \\
\hline 207 & 97 & 142 & 57 & 8 & DEATH VALLEY \\
\hline 207 & 97 & $141 \mathrm{~A}$ & 57 & 9 & DEATH VALLEY \\
\hline 203 & 95 & 141 & 57 & 10 & DEATH VALLEY \\
\hline 109 & 43 & 135 & & 1 & FRESNO \\
\hline 124 & 51 & & & 2 & FRESNO \\
\hline 100 & 38 & 136 & & 3 & FRESNO \\
\hline 72 & 22 & & & 4 & FRESNO \\
\hline 70 & 21 & & & 5 & FRESNO \\
\hline 73 & 23 & & & 1 & SANTA CRUZ \\
\hline 81 & 27 & & & 2 & SANTA CRUZ \\
\hline 119 & 48 & 132 & & 3 & SANTA CRUZ \\
\hline 75 & 24 & & & 4 & SANTA CRUZ \\
\hline 73 & 23 & & & 5 & SANTA CRUZ \\
\hline 112 & 44 & 133 & & 6 & SANTA CRUZ \\
\hline 77 & 25 & & & 1 & SANTA CRUZ \\
\hline 100 & 38 & & & 2 & SANTA CRUZ \\
\hline 114 & 46 & 90 & & 3 & SANTA CRUZ \\
\hline 98 & 37 & 92 & & 4 & SANTA CRUZ \\
\hline W & w & & & 5 & SANTA CRUZ \\
\hline 144 & 62 & 91 & & 6 & SANTA CRUZ \\
\hline 122 & 50 & 93 & & 7 & SANTA CRUZ \\
\hline 98 & 37 & 94 & & 8 & SANTA CRUZ \\
\hline 100 & 38 & 140 & & 1 & GOLDFIELD \\
\hline 70 & 21 & $127 \mathrm{~A}$ & & 1 & MARIPOSA \\
\hline 134 & 57 & 127 & & 2 & MARIPOSA \\
\hline 75 & 24 & & 56 & 3 & MARIPOSA \\
\hline 68 & 20 & & 56 & 4 & MARIPOSA \\
\hline 180 & 82 & 122 & 56 & 5 & MARIPOSA \\
\hline 134 & 57 & & 56 & 6 & MARIPOSA \\
\hline 127 & 53 & & 56 & 7 & MARIPOSA \\
\hline 199 & 93 & & 56 & 8 & MARIPOSA \\
\hline 167 & 75 & & 56 & 9 & MARIPOSA \\
\hline 120 & 49 & & 56 & 10 & MARIPOSA \\
\hline 171 & 77 & & 56 & 11 & MARIPOSA \\
\hline 72 & 22 & & 56 & 12 & MARIPOSA \\
\hline
\end{tabular}

1:63360 or 1:62500 (15-minute) or 1:24000 (7.5-minute) quadrangle

WAUCOBA WASH 15 NEW YORK BUTTE 15 KEELER 15

KEELER 15

TELESCOPE PEAK 15

HAIWEE RESERVOIR 15 HAIWEE RESERVOIR 15 HAIWEE RESERVOIR 15 KERN PEAK 15

HOCKETT PEAK 15

\section{MONACHE MTN 15}

SPRINGVILLE 7.5

GLOBE 7.5

ORTIGALITA PEAK 7.5

ORTIGALITA PEAK 7.5

MERCEY HOT SPRINGS 7.5

(CHOUNET RANCH 7.5)

LONOAK 7.5

SHERMAN PEAK 7.5

CHITTENDEN 7.5

(SEASIDE 7.5)

(BIC SUR 7.5)

PARAISO SPRINGS 7.5

(PARTINGTON RIDGE 7.5)

TASSAIARA HOT SPRINGS 7.5

\section{LOPEZ POINT 7.5}

LOPEZ POINT 7.5

UBEHEBE CRATER 15

BENTON 15

GLASS MOUNTAIN 15

CASA DIABLO MTN. 15

MT. MORRISON 15

MT. MORRISON 15

(MT. MORRISON 15)

(MT. MORRISON 15)

MT. MORRISON 15

(MT MORRISON 15)

(MT. MORRISON 15)

(MT. MORRISON 15)

(MT. MORRISON 15) 


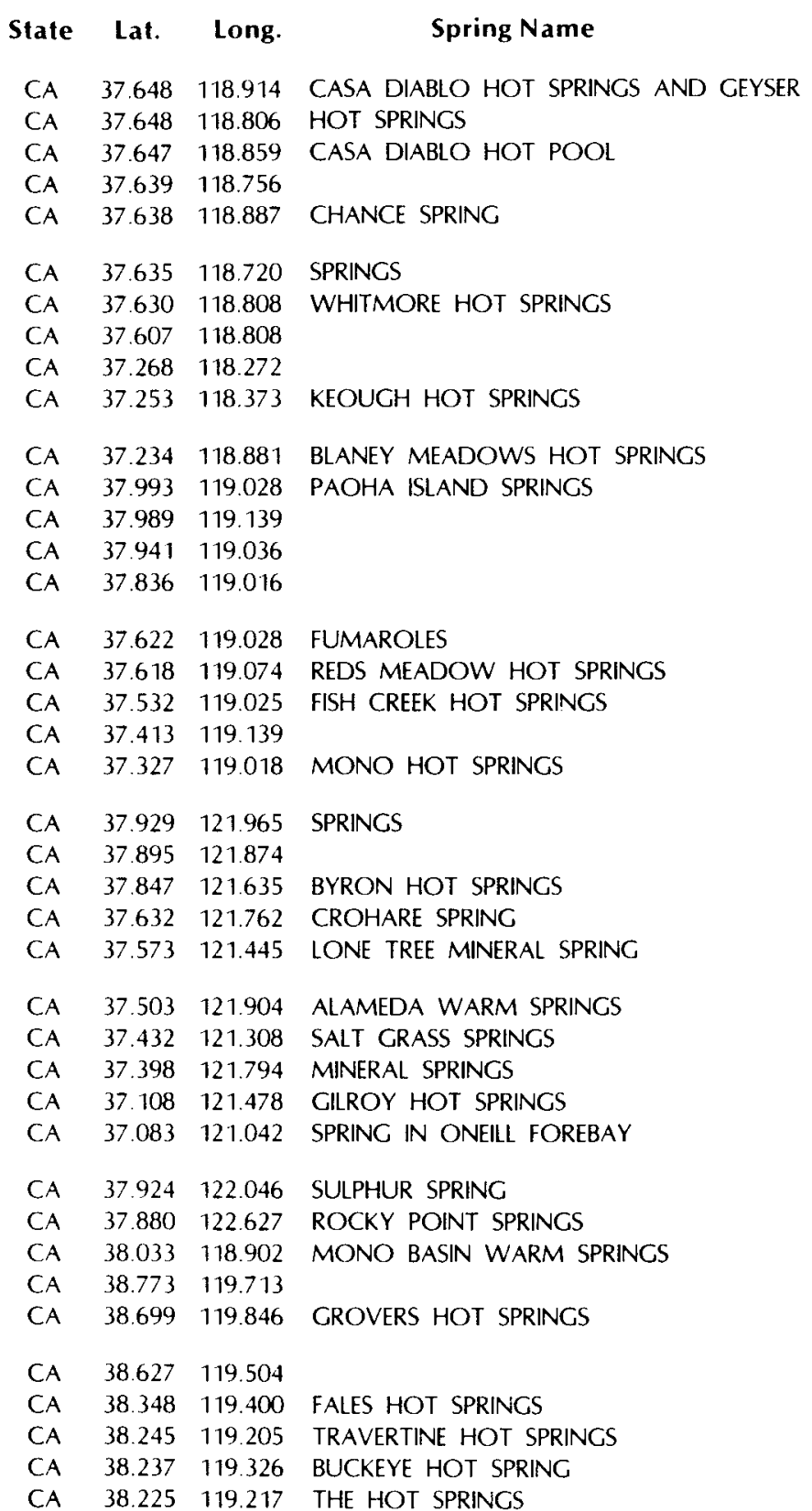

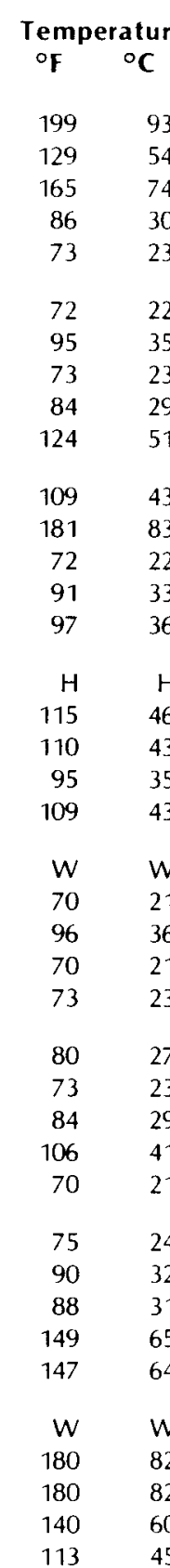

P.P. Circ. NOAA 1:250,000(AMS)map 492790

\begin{tabular}{|c|c|c|c|}
\hline 123 & 56 & 13 & MARIPOSA \\
\hline 125 & 56 & 14 & MARIPOSA \\
\hline \multirow[t]{4}{*}{124} & 56 & 15 & MARIPOSA \\
\hline & 56 & 16 & MARIPOSA \\
\hline & 56 & 17 & MARIPOSA \\
\hline & 56 & 18 & MARIPOSA \\
\hline \multirow[t]{3}{*}{126} & 56 & 19 & MARIPOSA \\
\hline & 56 & 20 & MARIPOSA \\
\hline & & 21 & MARIPOS \\
\hline 138 & & 22 & MARIPOSA \\
\hline 131 & & 23 & MARIPOS \\
\hline \multirow[t]{5}{*}{120} & & 1 & MARIPOS \\
\hline & & 2 & MARIPOS \\
\hline & & 3 & MARIPOSA \\
\hline & & 4 & MARIPOSA \\
\hline & & 5 & MARIPOSA \\
\hline 128 & & 6 & MARIPOS \\
\hline \multirow[t]{2}{*}{129} & & 7 & MARIPOSA \\
\hline & & 8 & MARIPOSA \\
\hline \multirow[t]{3}{*}{130} & & 9 & MARIPO \\
\hline & & 1 & SAN JOS \\
\hline & & 2 & SAN JOSE \\
\hline \multirow[t]{3}{*}{86} & & 3 & SAN JOSE \\
\hline & & 4 & SAN JOSE \\
\hline & & 5 & SAN IOSE \\
\hline \multirow[t]{2}{*}{87} & & 6 & SAN IOSE \\
\hline & & 7 & SAN IOS \\
\hline 88 & & 8 & SAN IOSE \\
\hline 89 & & 9 & SAN IOS \\
\hline
\end{tabular}

SAN FRANCISCO

SAN FRANCISCO

WALKER LAKE

WAIKER LAKE

WALKER LAKE

WALKER LAKE

WALKER LAKE

WALKER LAKE

WALKER LAKE

WALKER LAKE
1.63360 or $1: 62500$ (15-minute) or 1:24000 (7.5-minute) quadrangle

MT. MORRISON 15

MT. MORRISON 15

MT. MORRISON 15

(MT. MORRISON 15)

(MT. MORRISON 15)

CASA DIABLO MTN. 15

MT. MORRISON 15

(MT. MORRISON 15)

BISHOP 15

BISHOP 15

BLACKCAP MTN. 15 (MONO CRATERS 15) (MONO CRATERS 15) MONO CRATERS 15)

(DEVILS POSTPILE 15) DEVILS POSTPILE 15 DEVIILS POSTPILE 15 (KAISER PEAK 15) KAISER PEAK 15

CLAYTON 7.5 (ANTIOCH SOUTH 7.5) BYRON HOT SPRINGS 7.5 (LIVERMORE 7.5)

LONE TREE CREEK 7.5

NILES 7.5

COPPER MTN 75 CALAVERAS RESERVOIR 7.5 GILROY HOT SPRINGS 7.5 (SAN LUIS DAM 7.5)

(WAINUT CREEK 7.5) (BOLINAS 7.5) TRENCH CANYON 15 (MT. SIEGEL 15) MARKLEEVILLE 15

(TOPAZ LAKE 15) FALES HOT SPRINGS 7.5 BODIE 15 MATTERHORN PEAK 15 BODIE 15 


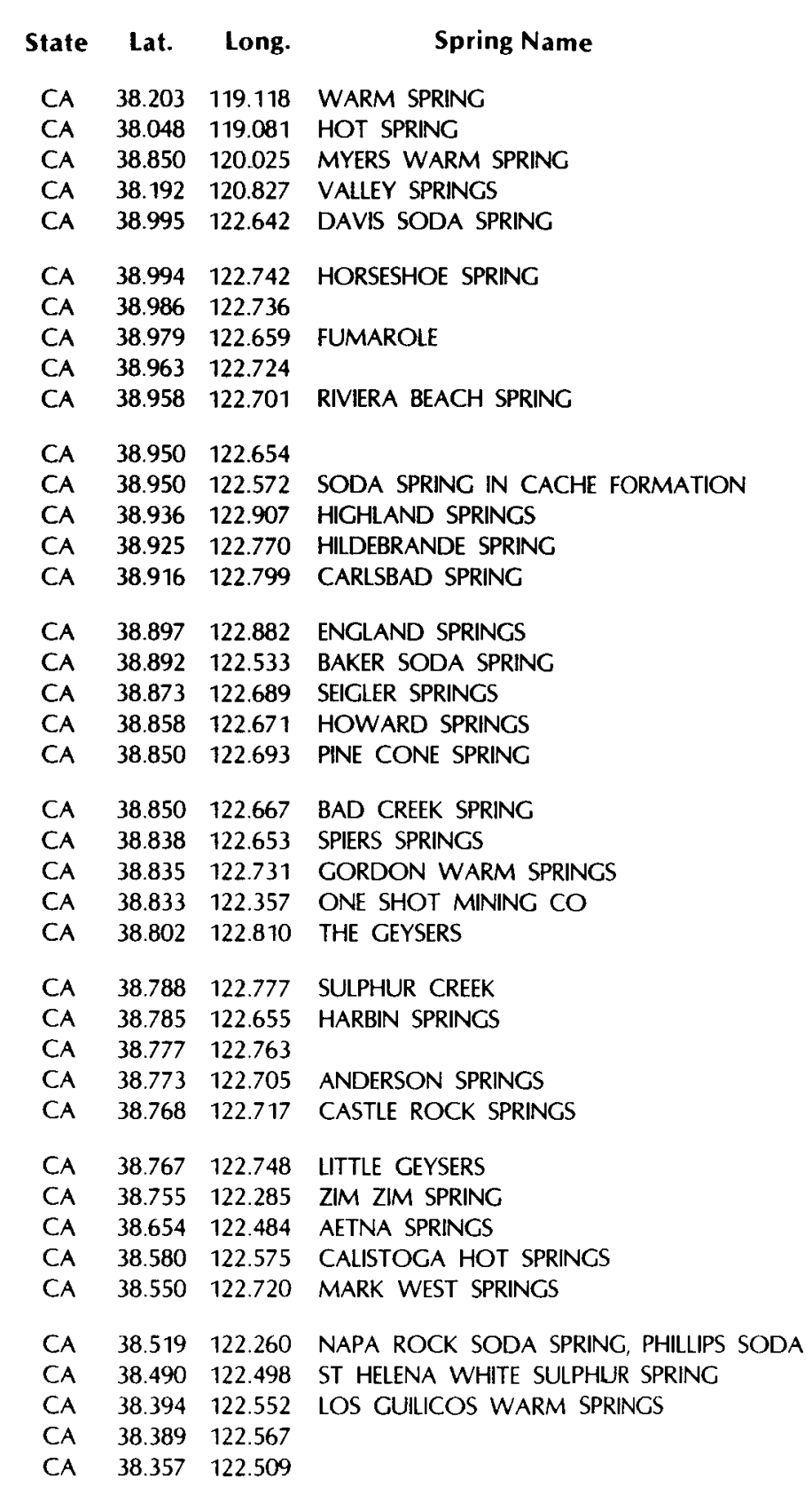

\begin{tabular}{|c|c|c|c|c|c|c|}
\hline \multicolumn{2}{|c|}{$\begin{array}{l}\text { Temperature } \\
{ }^{\circ} \mathrm{F}{ }^{\circ} \mathrm{C}\end{array}$} & $\begin{array}{l}\text { P.P. } \\
492\end{array}$ & $\begin{array}{l}\text { Circ. } \\
790\end{array}$ & NOAA & 1:250,000(AMS)map & $\begin{array}{l}1: 63360 \text { or } 1: 62500(15 \text {-minute }) \\
\text { or } 1: 24000(7.5 \text {-minute) quadrangle }\end{array}$ \\
\hline 77 & 25 & 119 & & 8 & WALKER LAKE & BODIE 15 \\
\hline 151 & 66 & & & 9 & WALKER LAKE & BODIE 15 \\
\hline 75 & 24 & & & 1 & SACRAMENTO & (ECHO LAKE 7.5) \\
\hline 75 & 24 & $113 \mathrm{~A}$ & & 2 & SACRAMENTO & VALLEY SPRINGS 7.5 \\
\hline 73 & 23 & & & 1 & SANTA ROSA & (CLEARLAKE HIGHLANDS 7.5) \\
\hline 108 & 42 & & 47 & 2 & SANTA ROSA & (CLEARLAKE HIGHLANDS 7.5) \\
\hline 95 & 35 & & 47 & 3 & SANTA ROSA & (CLEARLAKE HIGHLANDS 7.5) \\
\hline $\mathrm{H}$ & $\mathrm{H}$ & & & 4 & SANTA ROSA & (CLEARLAKE HIGHLANDS 7.5) \\
\hline W & W & & 47 & 5 & SANTA ROSA & (CLEARLAKE HIGHLANDS 7.5) \\
\hline 93 & 34 & & 47 & 6 & SANTA ROSA & (CLEARLAKE HIGHLANDS 7.5) \\
\hline w & w & & & 7 & SANTA ROSA & (CLEARLAKE HIGHLANDS 7.5) \\
\hline 72 & 22 & & & 8 & SANTA ROSA & (LOWER LAKE 7.5) \\
\hline 84 & 29 & 52 & & 9 & SANTA ROSA & HIGHLAND SPRINCS 7.5 \\
\hline 77 & 25 & & & 10 & SANTA ROSA & (KELSEYVILLE 7.5) \\
\hline 76 & 24 & 54 & 47 & 11 & SANTA ROSA & KELSEYVILLE 7.5 \\
\hline 76 & 24 & 53 & & 12 & SANTA ROSA & (HIGHLAND SPRINGS 7.5) \\
\hline 76 & 24 & & & 13 & SANTA ROSA & LOWER LAKE 7.5 \\
\hline 126 & 52 & 59 & 47 & 14 & SANTA ROSA & WHISPERING PINES 7.5 \\
\hline 113 & 45 & 58 & 47 & 15 & SANTA ROSA & WHISPERING PINES 7.5 \\
\hline 78 & 26 & & & 16 & SANTA ROSA & (WHISPERING PINES 7.5) \\
\hline 81 & 27 & & & 17 & SANTA ROSA & (WHISPERINC PINES 7.5) \\
\hline 79 & 26 & 61 & 47 & 18 & SANTA ROSA & (WHISPERING PINES 7.5) \\
\hline 97 & 36 & & & 19 & SANTA ROSA & (WHISPERING PINES 7.5) \\
\hline 72 & 22 & & & 20 & SANTA ROSA & (KNOXVILLE 7.5) \\
\hline 214 & 101 & 72 & 48 & 21 & SANTA ROSA & THE GEYSERS 7.5 \\
\hline 120 & 49 & 73 & 48 & 22 & SANTA ROSA & (THE GEYSERS 7.5) \\
\hline 120 & 49 & 64 & & 23 & SANTA ROSA & WHISPERING PINES 7.5 \\
\hline 120 & 49 & & 48 & 24 & SANTA ROSA & (THE GEYSERS 7.5) \\
\hline 108 & 42 & 63 & 48 & 25 & SANTA ROSA & WHISPERING PINES 7.5 \\
\hline 163 & 73 & 62 & 48 & 26 & SANTA ROSA & WHISPERING PINES 7.5 \\
\hline 210 & 99 & 74 & 48 & 27 & SANTA ROSA & (WHISPERING PINES 7.5) \\
\hline 70 & 21 & & & 28 & SANTA ROSA & (KNOXVILLE 7.5) \\
\hline 91 & 33 & 80 & & 29 & SANTA ROSA & AETNA SPRINGS 7.5 \\
\hline 172 & 78 & 81 & 50 & 30 & SANTA ROSA & CALISTOGA 7.5 \\
\hline 88 & 31 & 75 & & 31 & SANTA ROSA & MARK WEST SPRINCS 7.5 \\
\hline 78 & 26 & 83 & & 32 & SANTA ROSA & CHILES VALLEY 7.5 \\
\hline 97 & 36 & 82 & & 33 & SANTA ROSA & (RUTHERFORD 7.5) \\
\hline 88 & 31 & 76 & & 34 & SANTA ROSA & KENWOOD 7.5 \\
\hline 73 & 23 & 77 & & 35 & SANTA ROSA & KENWOOD 7.5 \\
\hline 70 & 21 & 78 & & 36 & SANTA ROSA & (GLEN ELLEN 7.5) \\
\hline
\end{tabular}




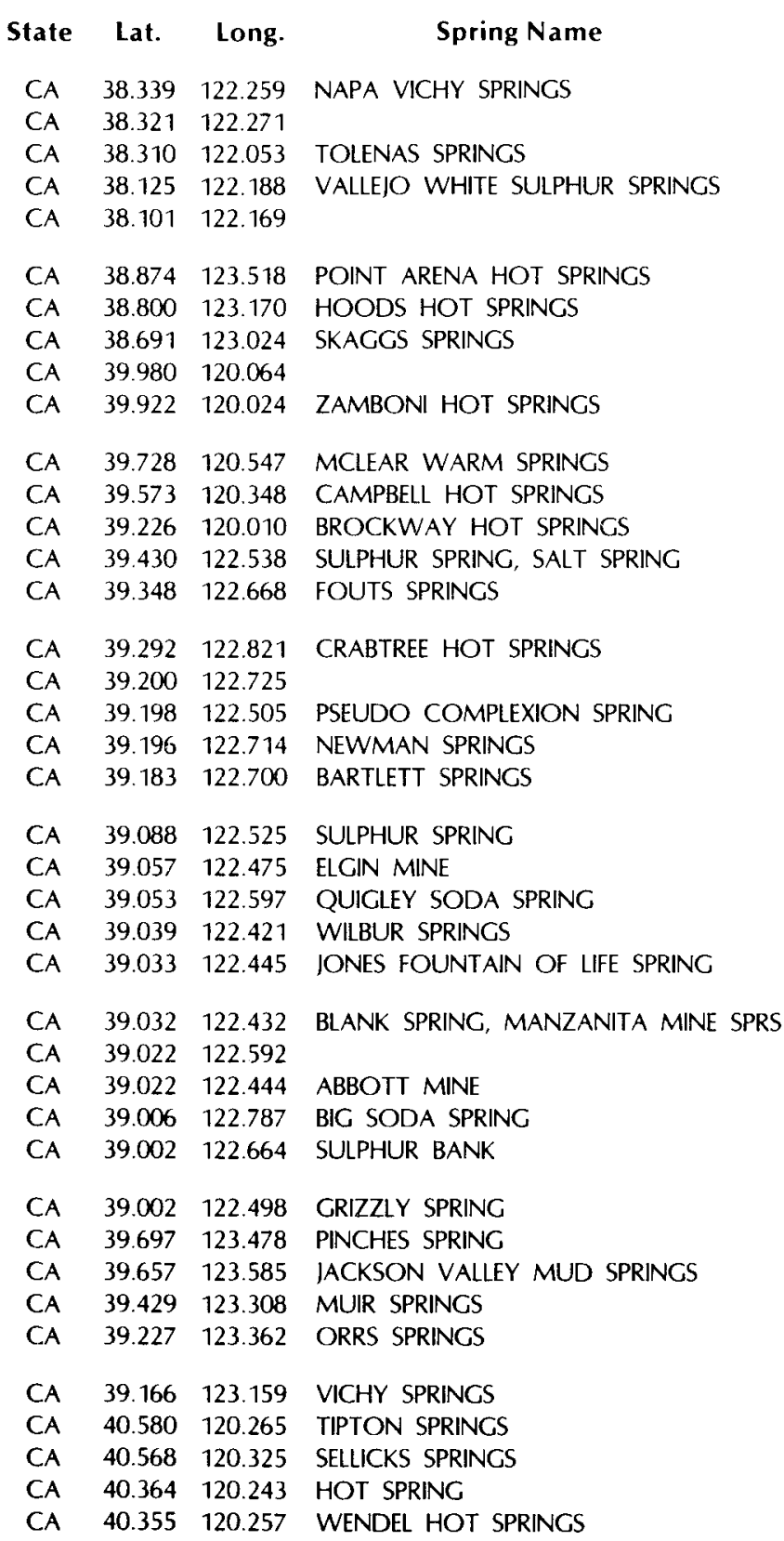

\begin{tabular}{|c|c|c|c|c|c|c|}
\hline \multicolumn{2}{|c|}{$\begin{array}{l}\text { Temperature } \\
{ }^{\circ} \mathrm{F} \quad{ }^{\circ} \mathrm{C}\end{array}$} & $\begin{array}{l}\text { P.P. } \\
492\end{array}$ & $\begin{array}{l}\text { Circ. } \\
790\end{array}$ & NOAA & 1:250,000(AMS)map & $\begin{array}{l}1: 63360 \text { or } 1: 62500(15 \text {-minute }) \\
\text { or } 1: 24000(7.5 \text {-minute) quadrangle }\end{array}$ \\
\hline 76 & 24 & & & 37 & SANTA ROSA & NAPA 7.5 \\
\hline 83 & 28 & & & 38 & SANTA ROSA & (NAPA 7.5) \\
\hline 68 & 20 & & & 39 & SANTA ROSA & FIARFIELD NORTH 7.5 \\
\hline 68 & 20 & & & 40 & SANTA ROSA & BENICIA 7.5 \\
\hline 73 & 23 & & & 41 & SANTA ROSA & (BENICIA 7.5) \\
\hline 111 & 44 & 47 & & 1 & SANTA ROSA & (GUALALA 7.5) \\
\hline 100 & 38 & 70 & & 2 & SANTA ROSA & (HOPLAND 15) \\
\hline 135 & 56 & 71 & 49 & 3 & SANTA ROSA & SKACCS SPRINCS 7.5 \\
\hline 108 & 42 & & & 1 & $\mathrm{CHICO}$ & (CONSTANTIA 7.5) \\
\hline $\mathrm{H}$ & $\mathrm{H}$ & & & 2 & $\mathrm{CHICO}$ & CONSTANTIA 7.5 \\
\hline 86 & 30 & 42 & & 3 & $\mathrm{CHICO}$ & SIERRA CITY 15 \\
\hline 111 & 44 & 43 & & 4 & $\mathrm{CHICO}$ & SIERRAVILLE 15 \\
\hline 131 & 55 & 44 & & 5 & $\mathrm{CHICO}$ & (KINCS BEACH 7.5) \\
\hline 75 & 24 & & & 1 & UKIAH & STONYFORD 7.5 \\
\hline 79 & 26 & $48 \mathrm{~A}$ & & 2 & UKIAH & FOUTS SPRINGS 7.5 \\
\hline 106 & 41 & 48 & & 3 & UKIAH & POTATO HILL 7.5 \\
\hline 90 & 32 & & & 4 & UKIAH & (CLEARLAKE OAKS 15) \\
\hline 81 & 27 & & & 5 & UKIAH & (CLEARLAKE OAKS 15) \\
\hline 94 & 35 & 49 & & 6 & UKIAH & CLEARLAKE OAKS 15 \\
\hline 144 & 62 & & & 7 & UKIAH & CLEARLAKE OAKS 15 \\
\hline 68 & 20 & & & 8 & UKIAH & CLEARLAKE OAKS 15 \\
\hline 156 & 69 & 69 & & 9 & UKIAH & WILBUR SPRINGS 15 \\
\hline 82 & 28 & & & 10 & UKIAH & CLEARLAKE OAKS 15 \\
\hline 153 & 67 & 68 & 44 & 11 & UKIAH & WILBUR SPRINCS 15 \\
\hline 142 & 61 & & & 12 & UKIAH & WILBUR SPRINGS 15 \\
\hline 112 & 45 & 66 & 44 & 13 & UKIAH & WILBUR SPRINCS 15 \\
\hline 81 & 27 & & & 14 & UKIAH & CLEARLAKE OAKS 15 \\
\hline 86 & 30 & & & 15 & UKIAH & WILBUR SPRINCS 15 \\
\hline 90 & 32 & 55 & 47 & 16 & UKIAH & LUCERNE 7.5 \\
\hline 156 & 69 & 57 & 46 & 17 & UKIAH & CLEARLAKE OAKS 7.5 \\
\hline 68 & 20 & & & 18 & UKIAH & WILBUR SPRINGS 15 \\
\hline 70 & 21 & $45 \mathrm{~A}$ & & 1 & UKIAH & LAYTONVILLE 7.5 \\
\hline 80 & 27 & & & 2 & UKIAH & CAHTO PEAK 7.5 \\
\hline 68 & 20 & & & 3 & UKIAH & (WILLITS 15) \\
\hline 104 & 40 & 45 & & 4 & UKIAH & BOONVILLE 15 \\
\hline 85 & 29 & 46 & & 5 & UKIAH & UKIAH 7.5 \\
\hline 70 & 21 & $29 \mathrm{~A}$ & & 1 & SUSANVILLE & KARLO 15 \\
\hline 72 & 22 & & & 2 & SUSANVILLE & KARLO 15 \\
\hline 204 & 96 & & 42 & 3 & SUSANVILLE & WENDEL 15 \\
\hline 205 & 96 & 30 & 42 & 4 & SUSANVILLE & LITCHFIELD 15 \\
\hline
\end{tabular}


State Lat. Long.

\section{Spring Name}

$\begin{array}{llll}C A & 40.302 & 120.195 & \text { AMEDEE HOT SPRINCS }\end{array}$

CA $40.245 \quad 120.007$ HICH ROCK SPRINC

$\begin{array}{llll}C A & 40.142 & 120.935 & \text { INDIAN VALLEY HOT SPRINGS }\end{array}$

CA $40.457 \quad 121.545$ MILL CREEK SPRINCS

CA $\quad 40.455 \quad 121.501$ BUMPASS HELL

40.447121 .536 SULPHUR WORKS, TOPHET HOT SPRINCS

$40.444 \quad 121.409$ DRAKESBAD

40.440121 .434 DEVILS KITCHEN

40.440121 .420 HOT SPRINGS VALLEY

40.434121 .399 BOILING SPRINGS LAKE

$40.421 \quad 121.375$ TERMINAL GEYSER

$40.393 \quad 121.507$ GROWLER HOT SPRINC

40.382121 .513 MORGAN HOT SPRINC

40.019121 .036 SPRINC

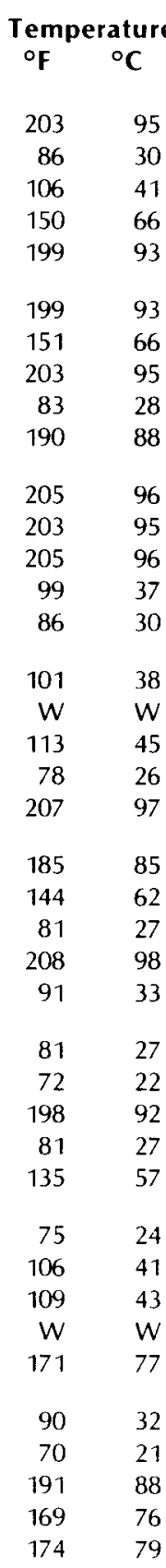

P.P. Circ. NOAA 1:250,000(AMS)map 492790

\begin{tabular}{|c|c|c|c|}
\hline 31 & 42 & 5 & SUSANVILLE \\
\hline 32 & & 6 & SUSANVILLE \\
\hline 39 & & 7 & SUSANVILLE \\
\hline 25 & 40 & 1 & SUSANVILLE \\
\hline 27 & 40 & 2 & SUSANVILLE \\
\hline 26 & 40 & 3 & SUSANVILLE \\
\hline 36 & 40 & 4 & SUSANVILLE \\
\hline 34 & 40 & 5 & SUSANVILLE \\
\hline 35 & 40 & 6 & SUSANVILLE \\
\hline 37 & 40 & 7 & SUSANVILLE \\
\hline 38 & 40 & 8 & SUSANVILLE \\
\hline & 41 & 9 & SUSANVILLE \\
\hline 33 & 41 & 10 & SUSANVILLE \\
\hline 40 & & 11 & SUSANVILLE \\
\hline $45 B$ & & 1 & REDDING \\
\hline & & 2 & REDDING \\
\hline & & 1 & ALTURAS \\
\hline & 34 & 2 & ALTURAS \\
\hline 4 & & 3 & ALTURAS \\
\hline 14 & 35 & 4 & ALTURAS \\
\hline 16 & 35 & 5 & ALTURAS \\
\hline 17 & 35 & 6 & ALTURAS \\
\hline & & 7 & ALTURAS \\
\hline 18 & 35 & 8 & ALTURAS \\
\hline 6 & & 9 & ALTURAS \\
\hline 7 & & 10 & ALTURAS \\
\hline 10 & & 11 & ALTURAS \\
\hline 8 & 38 & 12 & ALTURAS \\
\hline & & 13 & ALTURAS \\
\hline 20 & & 14 & ALTURAS \\
\hline & & 15 & ALTURAS \\
\hline 21 & & 16 & ALTURAS \\
\hline & & 17 & ALTURAS \\
\hline & & 18 & ALTURAS \\
\hline & 36 & 19 & ALTURAS \\
\hline 22 & & 20 & ALTURAS \\
\hline & & 21 & ALTURAS \\
\hline $3 \mathrm{~A}$ & & 1 & ALTURAS \\
\hline 11 & & 2 & ALTURAS \\
\hline 28 & 37 & 3 & ALTURAS \\
\hline
\end{tabular}

1:63360 or 1:62500 (15-minute)

or 1:24000 (7.5-minute) quadrangle

WENDEL 15

DOYLE 15

GREENVILLE 15

(LASSEN PEAK 15)

LASSEN PEAK 15

LASSEN PEAK 15

MT. HARKNESS 15

MT. HARKNESS 15

(MT HARKNESS 15)

MT. HARKNESS 15

MT. HARKNESS 15

LASSEN PEAK 15

LASSEN PEAK 15

ALMANOR 15

TUSCAN SPRINGS 7.5

COLD FORK 7.5

STEELE SWAMP 15

FORT BIDWELL 15

STEELE SWAMP 15

CEDARVILLE 15

CEDARVILLE 15

CEDARVILLE 15

(BIG SAGE RESERVOIR 15)

CEDARVILLE 15

ALTURAS 15

CANBY 15

ALTURAS 15

CANBY 15

(ALTURAS 15)

EAGLEVILLE 7.5

ALTURAS 15

(SNAKE LAKE 7.5)

(SNAKE LAKE 7.5)

TULE MOUNTAIN 7.5

(TULE MOUNTAIN 7.5)

(SNAKE LAKE 7.5)

TULE MOUNTAIN 75

MEDICINE LAKE 15

FALL RIVER MILLS 15

BIEBER 15 


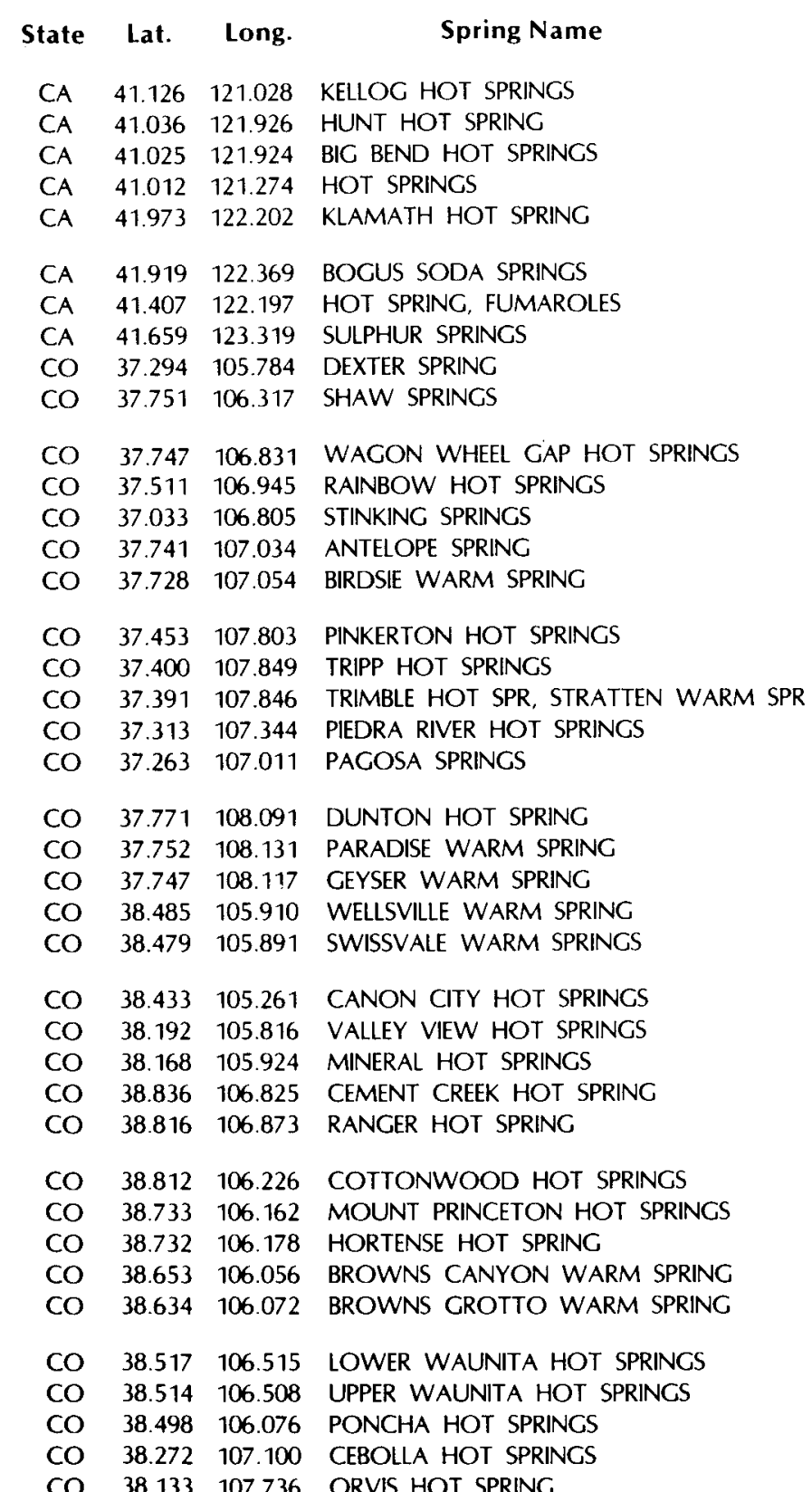

\begin{tabular}{rr}
\multicolumn{1}{c}{$\begin{array}{c}\text { Temperature } \\
\text { OF }\end{array}$} & ${ }^{\circ} \mathbf{C}$ \\
172 & 78 \\
136 & 58 \\
180 & 82 \\
H & H \\
156 & 69 \\
76 & 24 \\
76 & 84 \\
183 & 84 \\
84 & 29 \\
68 & 20 \\
86 & 30 \\
135 & 57 \\
104 & 40 \\
81 & 27 \\
90 & 32 \\
86 & 30 \\
90 & 32 \\
111 & 44 \\
97 & 36 \\
108 & 42 \\
136 & 58 \\
108 & 42 \\
115 & 46 \\
82 & 28 \\
91 & 33 \\
82 & 28 \\
104 & 40 \\
99 & 37 \\
140 & 60 \\
77 & 25 \\
81 & 27 \\
136 & 58 \\
133 & 56 \\
181 & 83 \\
77 & 25 \\
73 & 23 \\
167 & 75 \\
176 & 80 \\
160 & 7 \\
106 & 4 \\
126 & 52 \\
&
\end{tabular}

P.P. Circ. NOAA 1:250,000(AMS)map 492790

$\begin{array}{rrrl}29 & & 4 & \text { ALTURAS } \\ 23 & & 5 & \text { ALTURAS } \\ 24 & 39 & 6 & \text { ALTURAS } \\ & & 7 & \text { ALTURAS } \\ 2 & & 1 & \text { WEED } \\ & & & \\ 2 A & & 2 & \text { WEED } \\ 3 & & 3 & \text { WEED } \\ 1 & & 1 & \text { WEED } \\ 44 & & 1 & \text { TRINIDAD } \\ 33 & & 1 & \text { DURANGO } \\ & & & \\ 31 & 79 & 2 & \text { DURANGO } \\ 32 & & 3 & \text { DURANGO } \\ 41 & & 4 & \text { DURANCO } \\ & & 1 & \text { DURANGO } \\ & & 2 & \text { DURANGO } \\ 34 & & 3 & \text { DURANGO } \\ 35 & & 4 & \text { DURANGO } \\ 36 & & 5 & \text { DURANGO } \\ & & 6 & \text { DURANCO } \\ 39 & & 7 & \text { DURANGO } \\ 29 & & 1 & \text { CORTEZ } \\ 29 & 78 & 2 & \text { CORTEZ } \\ & & 3 & \text { CORTEZ } \\ 22 & & 1 & \text { PUEBLO } \\ & & 2 & \text { PUEBLO } \\ 22 A & & 3 & \text { PUEBLO } \\ 24 & & 4 & \text { PUEBLO } \\ 23 & & 5 & \text { PUEBLO } \\ 13 & & 1 & \text { MONTROSE } \\ 12 & & 2 & \text { MONTROSE } \\ 19 & & 3 & \text { MONTROSE } \\ 20 & 74 & 4 & \text { MONTROSE } \\ & 74 & 5 & \text { MONTROSE } \\ & & 6 & \text { MONTROSE } \\ & & 7 & \text { MONTROSE } \\ & 76 & 8 & \text { MONTROSE } \\ 14 & 76 & 9 & \text { MONTROSE } \\ 21 & 75 & 10 & \text { MONTROSE } \\ 15 & 77 & 1 & \text { MONTROSE } \\ 27 & & 2 & \text { MONTROSE } \\ & & & \end{array}$

1:63360 or 1:62500 (15-minute) or 1:24000 (7.5-minute) quadrangle

BIEBER 15

BIC BEND 15

BIC BEND 15

FALL RIVER MILLS 15

MACDOEL 15

COPCO 15

SHASTA 15

UKONOM LAKE 15

TWIN MOUNTAINS SE 7.5

SPAR CITY 15

SPAR CITY 15

CHROMO 15

WORKMAN CREEK 7.5

(WORKMAN CREEK 7.5)

(HERMOSA 7.5)

(HERMOSA 7.5)

(HERMOSA 7.5)

(DEVIL MOUNTAIN 7.5)

PAGOSA SPRINGS 7.5

DOLORES PEAK 7.5

GROUNDHOG MOUNTAIN 7.5)

(RICO 7.5)

(HOWARD 15)

(HOWARD 15)

(ROYAL GORGE 15)

VALLLEY VIEW HOT SPRINCS 7.5

VILLA GROVE 7.5

CEMENT MTN. 7.5

CEMENT MTN. 7.5

BUENA VISTA 15

PONCHA SPRINCS 15

PONCHA SPRINGS 15

(PONCHA SPRINGS 15)

(PONCHA SPRINCS 15)

(PITKIN 7.5)

PITKIN 7.5

BONANZA 15

POWDERHORN 7.5

DALLAS 7.5 


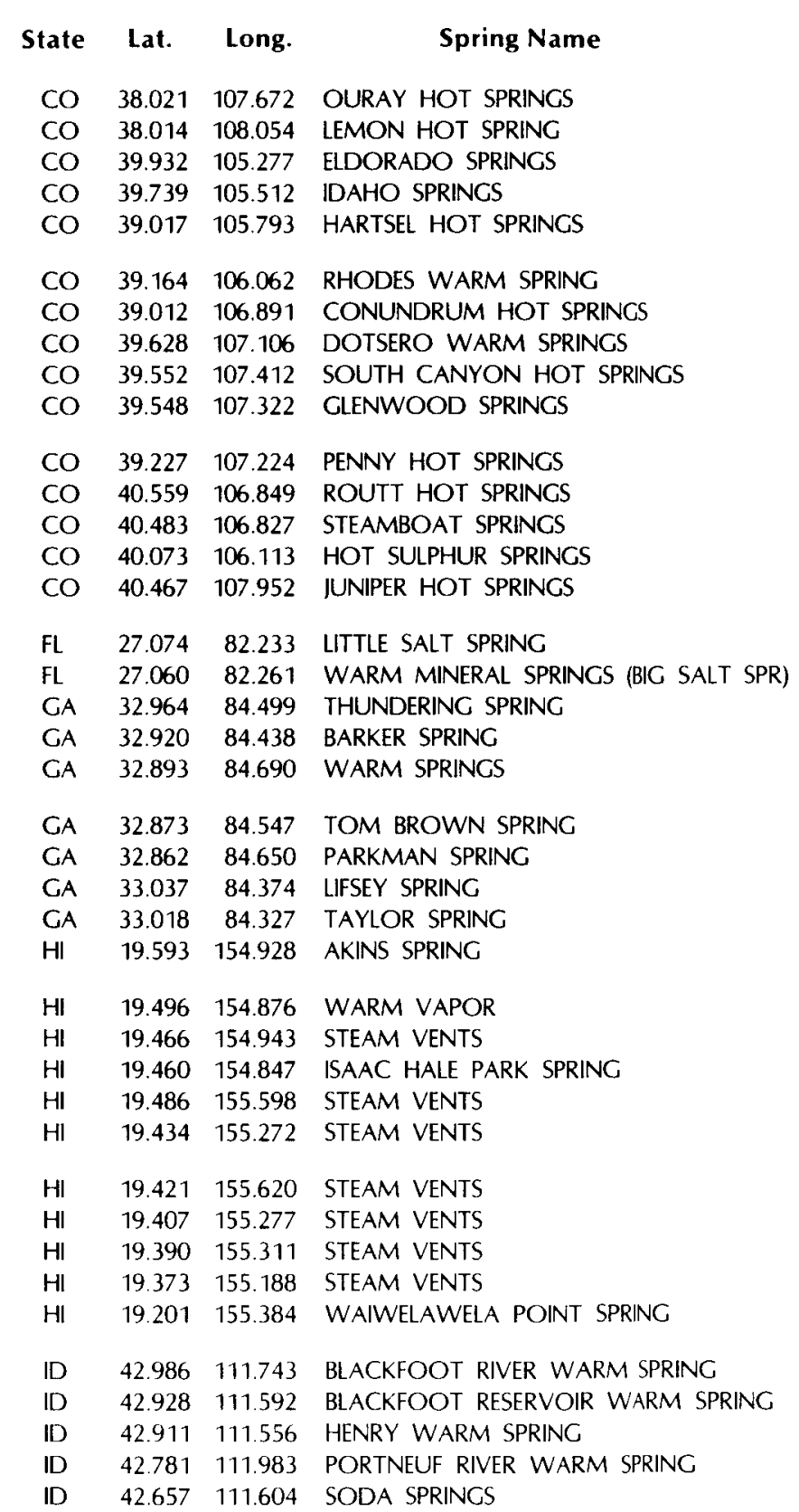

\begin{tabular}{|c|c|c|c|c|c|}
\hline Temp & ture & P.P. & Circ. & NOAA & $1: 250,000$ (AMS)map \\
\hline${ }^{\circ} \mathbf{F}$ & C & 492 & 790 & & \\
\hline 156 & 69 & 28 & & 3 & MONTROSE \\
\hline 91 & 33 & 26 & & 1 & MOAB \\
\hline 79 & 26 & 4 & & 1 & DENVER \\
\hline 115 & 46 & 5 & & 2 & DENVER \\
\hline 126 & 52 & 17 & & 3 & DENVER \\
\hline 75 & 24 & 16 & & 1 & LEADVILLE \\
\hline 100 & 38 & 9 & & 2 & LEADVILLE \\
\hline 90 & 32 & 7 & & 1 & LEADVILLE \\
\hline 118 & 48 & & & 2 & LEADVILLE \\
\hline 124 & 51 & 6 & & 3 & LEADVILLE \\
\hline 133 & 56 & & 73 & 4 & LEADVILLE \\
\hline 147 & 64 & 2 & 72 & 1 & CRAIG \\
\hline 102 & 39 & $2 \mathrm{~A}$ & & 2 & CRAIG \\
\hline 111 & 44 & 3 & & 3 & CRAIG \\
\hline 100 & 38 & 1 & & 1 & CRAIG \\
\hline 81 & 27 & & & 1 & TAMPA \\
\hline 86 & 30 & 1 & & 2 & TAMPA \\
\hline 75 & 24 & 3 & & 1 & PHENIX CITY \\
\hline 73 & 23 & 5 & & 2 & PHENIX CITY \\
\hline 88 & 31 & 6 & & 3 & PHENIX CITY \\
\hline 68 & 20 & 8 & & 4 & PHENIX CITY \\
\hline 77 & 25 & 7 & & 5 & PHENIX CITY \\
\hline 79 & 26 & 1 & & 1 & ATLANTA \\
\hline 75 & 24 & 2 & & 2 & ATLANTA \\
\hline 84 & 29 & & & 1 & HAWAll \\
\hline W & w & & & 2 & HAWAIl \\
\hline $\mathrm{H}$ & $\mathrm{H}$ & & & 3 & HAWAII \\
\hline 97 & 36 & 7 & & 4 & HAWAII \\
\hline $\mathrm{H}$ & $H$ & & & 1 & HAWAII \\
\hline $\mathrm{H}$ & $\mathrm{H}$ & & & 2 & HAWAll \\
\hline $\mathrm{H}$ & H & & & 3 & HAWAIl \\
\hline $\mathrm{H}$ & $\mathrm{H}$ & & & 4 & HAWAll \\
\hline $\mathrm{H}$ & $\mathrm{H}$ & & & 5 & HAWAIl \\
\hline $\mathrm{H}$ & $\mathrm{H}$ & & & 6 & HAWAII \\
\hline 90 & 32 & 8 & & 7 & HAWAII \\
\hline 79 & 26 & & & 1 & PRESTON \\
\hline 72 & 22 & & & 2 & PRESTON \\
\hline 86 & 30 & 192 & & 3 & PRESTON \\
\hline 93 & 34 & & & 4 & PRESTON \\
\hline 82 & 28 & 193 & & 5 & PRESTON \\
\hline
\end{tabular}

1:63360 or $1: 62500$ (15-minute) or 1:24000 (7.5-minute) quadrangle

(OURAY 7.5)

(PLACERVILLE 7.5)

ELDORADO SPRINGS 7.5

IDAHO SPRINGS 7.5

HARTSEL 7.5

FAIRPLAY WEST 7.5

MAROON BELLS 7.5

(GLENWOOD SPRINGS 15)

(STORM KING MOUNTAIN 7.5)

GLENWOOD SPRINCS 7.5

REDSTONE 7.5

ROCKY PEAK 7.5

STEAMBOAT SPRINCS 7.5

HOT SULPHUR SPRINCS 15

JUNIPER HOT SPRINCS 7.5

\section{MURDOCK 7.5}

MYAKKA RIVER 7.5

SUNSET VILLAGE 7.5

SUNSET VILLAGE 7.5

WARM SPRINGS 7.5

MANCHESTER 7.5

SHILOH 7.5

ZEBULON 7.5

ZEBULON 7.5

(PAHOA NORTH 7.5)

(PAHOA SOUTH 7.5)

(PAHOA SOUTH 7.5)

(KAPOHO 7.5)

(MAUNA LOA 7.5)

KILAUEA CRATER 7.5

(MAUNA LOA 7.5)

(KILAUEA CRATER 7.5)

(MAKAOPUHI CRATER 7.5)

(PAHALA 7.5)

(HENRY 15)

(HENRY 15)

HENRY 15

(PORTNEUF 15)

SODA SPRINGS 15 


\begin{tabular}{|c|c|c|c|}
\hline State & Lat. & Long. & Spring Name \\
\hline ID & 42.655 & 111.643 & STEAMBOAT HOT SPRINC \\
\hline ID & 42.426 & 111.378 & PESCADERO WARM SPRING \\
\hline ID & 42.374 & 111.726 & MOUND VALLEY WARM SPRING \\
\hline ID & 42.337 & 111.726 & TREASURETON HOT SPRINGS \\
\hline ID & 42.333 & 111.716 & CIEVELAND HOT SPRINCS \\
\hline ID & 42.308 & 111.707 & MAPLE GROVE HOT SPRINGS \\
\hline ID & 42.133 & 111.928 & BATTLE CREEK HOT SPRINGS \\
\hline ID & 42.119 & 111.928 & SQUAW HOT SPRINCS \\
\hline ID & 42.115 & 111.264 & BEAR LAKE HOT SPRINCS \\
\hline ID & 42.957 & 112.458 & TADPOLE WARM SPRING \\
\hline ID & 42.725 & 112.872 & INDIAN SPRINGS \\
\hline ID & 42.621 & 112.008 & LAVA HOT SPRINGS \\
\hline ID & 42.545 & 112.906 & ROCKLAND WARM SPRINCS \\
\hline ID & 42.388 & 112.085 & DOWNATA HOT SPRINCS \\
\hline ID & 42.339 & 112.436 & KENT WARM SPRING \\
\hline ID & 42.173 & 112.239 & MALAD WARM SPRING \\
\hline ID & 42.156 & 112.348 & PLEASANTVIEW WARM SPRINCS \\
\hline ID & 42.056 & 112.246 & WOODRUFF HOT SPRING \\
\hline ID & 42.025 & 112.226 & PRICES WARM SPRING \\
\hline ID & 42.625 & 113.107 & LAKE WALCOTT HOT SPRING \\
\hline ID & 42.449 & 113.511 & MARSH GL \\
\hline ID & 42.447 & 113.523 & MARSH CREEK HOT SPRINC \\
\hline ID & 42.286 & 113.446 & RICE SPRING, WARD SPRING \\
\hline ID & 42.240 & 113.588 & SEARS SPRING \\
\hline ID & 42.173 & 113.861 & OAKLEY HOT SPRINC \\
\hline ID & 42.107 & 113.390 & BLM WELL (FRAZIER HOT SPRING) \\
\hline ID & 42.102 & 113.632 & DURFEE SPRING \\
\hline ID & 42.085 & 113.564 & GRAPE CREEK WARM SPRING \\
\hline ID & 42.704 & 114.856 & SALMON FALLS HOT SPRING \\
\hline ID & 42.692 & 114.859 & HOT SULPHUR (MIRACLE HOT) SPRINCS \\
\hline ID & 42.688 & 114.826 & BANBURY HOT SPI \\
\hline ID & 42.637 & 114.892 & POISON SPRINGS \\
\hline ID & 42.405 & 114.142 & ARTESIAN CITY HOT SPRINCS \\
\hline ID & 42.337 & 114.509 & NAT-SOO-PAH WARM SPRINC \\
\hline ID & 42.015 & 114.237 & THOROUGHBRED WARM SPRINCS \\
\hline ID & 42.013 & 114.504 & MACIC HC \\
\hline ID & 42.798 & 115.742 & BRUNEAU HOT SPRINC \\
\hline ID & 42.797 & 115.723 & BAT HOT SPRING, PENCE HOT SPRING \\
\hline ID & 42.779 & 115.715 & BUCKAROO HOT SPRINC \\
\hline ID & 42.767 & 115.725 & LOWER INDIAN BATHTUB HOT \\
\hline
\end{tabular}

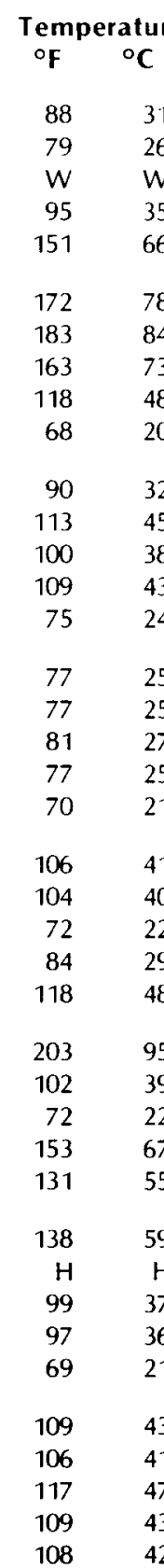

P.P. Circ. NOAA 1:250,000(AMS)map 492790

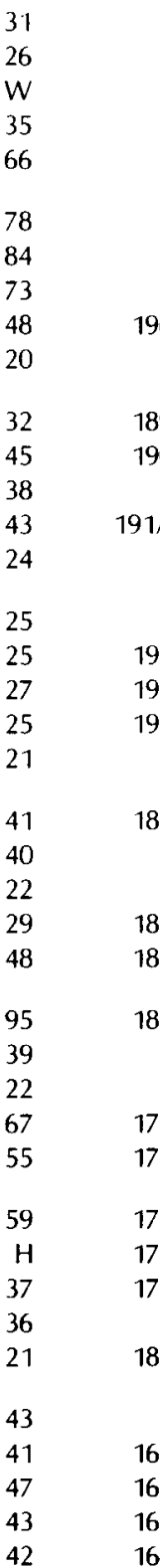

\begin{tabular}{|c|c|c|}
\hline & 6 & PRESTON \\
\hline & 7 & PRESTON \\
\hline & 8 & PRESTON \\
\hline & 9 & PRESTON \\
\hline & 10 & PRESTON \\
\hline 118 & 11 & PRESTON \\
\hline 120 & 12 & PRESTON \\
\hline \multirow[t]{18}{*}{121} & 13 & PRESTON \\
\hline & 14 & PRESTON \\
\hline & 1 & POCATELLO \\
\hline & 2 & POCATELLO \\
\hline & 3 & POCATELLO \\
\hline & 4 & POCATELLO \\
\hline & 5 & POCATELLO \\
\hline & 6 & POCATELLO \\
\hline & 7 & POCATELLO \\
\hline & 8 & POCATELLO \\
\hline & 9 & POCATELLO \\
\hline & 10 & POCATELLO \\
\hline & 1 & POCATELLO \\
\hline & 2 & POCATELLO \\
\hline & 3 & POCATELLO \\
\hline & 4 & POCATELLO \\
\hline & 5 & POCATELLO \\
\hline & 6 & POCATELLO \\
\hline \multirow[t]{5}{*}{115} & 7 & POCATELLO \\
\hline & 8 & POCATELLO \\
\hline & 9 & POCATELLO \\
\hline & 1 & IWIN FALLS \\
\hline & 2 & TWIN FALLS \\
\hline \multirow[t]{10}{*}{114} & 3 & TWIN FALLS \\
\hline & 4 & TWIN FALLS \\
\hline & 5 & TWIN FALLS \\
\hline & 6 & TWIN FALLS \\
\hline & 7 & TWIN FALLS \\
\hline & 8 & TWIN FALLS \\
\hline & 1 & TWIN FALLS \\
\hline & 2 & TWIN FALLS \\
\hline & 3 & TWIN FALLS \\
\hline & 4 & TWIN FALLS \\
\hline
\end{tabular}

1:63360 or 1:62500 (15-minute) or 1:24000 (7.5-minute) quadrangle

(SODA SPRINGS 15)

NOUNAN 7.5

ONEIDA NARROWS RESERVOIR 7.5

ONEIDA NARROWS RESERVOIR 75

ONEIDA NARROWS RESERVOIR 75

ONEIDA NARROWS RESERVOIR 7.5 BANIDA 7.5

WESTON 7.5

BEAR LAKE NORTH 7.5

POCATELLO NORTH 7.5

INDIAN SPRINGS 7.5

LAVA HOT SPRINGS 7.5

ROCKLAND WEST 7.5

DOWNEY EAST 7.5

(DANIELS 7.5)

(MALAD CITY EAST 7.5)

MALAD CITY WEST 7.5

HENDERSON CREEK 7.5

(HENDERSON CREEK 7.5)

(YALE 15)

ALBION 7.5

(ALBION 7.5)

NIBBS CREEK 7.5

(ELBA 7.5)

BASIN 7.5

CHOKECHERRY CANYON 75

(ALMO 7.5)

IIM SAGE CANYON 7.5

(THOUSAND SPRINCS 7.5)

THOUSAND SPRINCS 7.5

THOUSAND SPRINGS 7.5

BUHL 15

(MURTAUCH 7.5)

HOLLISTER 7.5

HOT SPRING 7.5

HOT SPRING 7.5

(HOT SPRINC 7.5) 


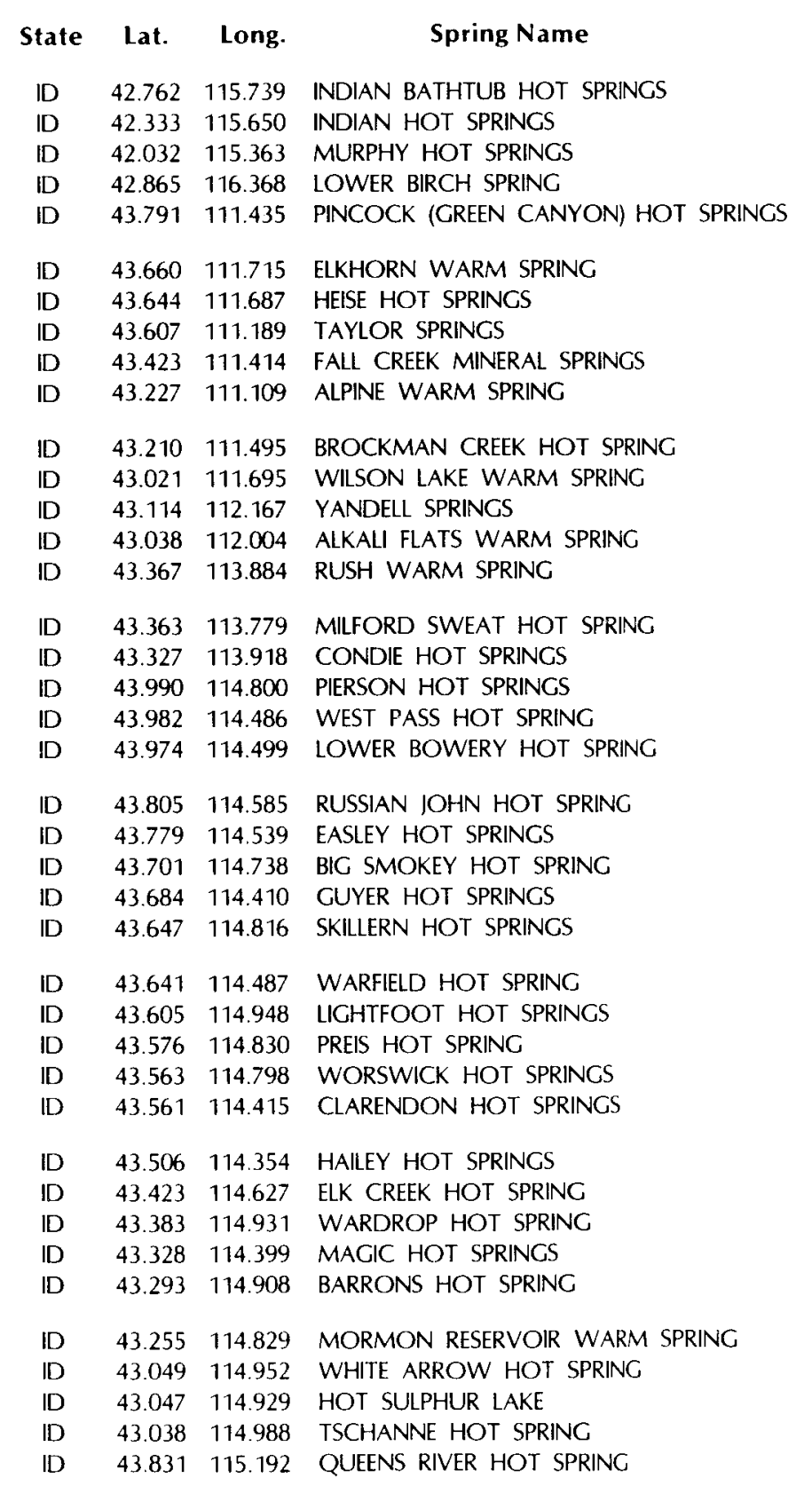

\begin{tabular}{|c|c|c|c|c|c|}
\hline \multicolumn{2}{|c|}{ Temperature } & \multirow{2}{*}{$\begin{array}{l}\text { P.P. } \\
492\end{array}$} & \multirow{2}{*}{$\begin{array}{c}\text { Circ. } \\
790\end{array}$} & \multirow{2}{*}{ NOAA } & \multirow{2}{*}{$1: 250,000(A M S)$ map } \\
\hline & & & & & \\
\hline 99 & 37 & 167 & & 5 & TWIN FALLS \\
\hline 160 & 71 & $169 \mathrm{~A}$ & & 6 & TWIN FALLS \\
\hline 126 & 52 & $169 \mathrm{~B}$ & 103 & 7 & TWIN FALLS \\
\hline 77 & 25 & & & 1 & JORDAN VALLEY \\
\hline 111 & 44 & 153 & & 1 & DRIGGS \\
\hline 72 & 22 & & & 2 & DRIGGS \\
\hline 120 & 49 & 152 & & 3 & DRIGCS \\
\hline 68 & 20 & & & 4 & DRIGGS \\
\hline 77 & 25 & 154 & & 5 & DRIGGS \\
\hline 99 & 37 & & & 6 & DRIGGS \\
\hline 95 & 35 & & & 7 & DRICGS \\
\hline 86 & 30 & & & 8 & DRIGGS \\
\hline 90 & 32 & 157 & & 1 & IDAHO FALLS \\
\hline 93 & 34 & & & 2 & IDAHO FALLS \\
\hline 72 & 22 & & & 1 & IDAHO FALLS \\
\hline 111 & 44 & & & 2 & IDAHO FALLS \\
\hline 124 & 51 & 147 & & 3 & IDAHO FALLS \\
\hline 109 & 43 & 102 & & 1 & HAILEY \\
\hline 124 & 51 & 103 & & 2 & HAILEY \\
\hline 129 & 54 & & & 3 & HAILEY \\
\hline 100 & 38 & 140 & & 4 & HAILEY \\
\hline 100 & 38 & 141 & & 5 & HAILEY \\
\hline $\mathrm{H}$ & $\mathrm{H}$ & 132 & & 6 & HAILEY \\
\hline 158 & 70 & 142 & & 7 & HAlLEY \\
\hline 140 & 60 & 133 & & 8 & HAILEY \\
\hline 124 & 51 & 143 & & 9 & HAILEY \\
\hline 133 & 56 & 134 & & 10 & HAllEY \\
\hline 106 & 41 & 135 & & 11 & HAllEY \\
\hline 180 & 82 & 136 & 110 & 12 & HAILEY \\
\hline 126 & 52 & 151 & & 13 & HAILEY \\
\hline 131 & 55 & 145 & & 14 & HAILEY \\
\hline 126 & 52 & 138 & & 15 & HAILEY \\
\hline 147 & 64 & 137 & 111 & 16 & HAllEY \\
\hline 163 & 73 & & 109 & 17 & HAILEY \\
\hline 167 & 75 & 139 & 112 & 18 & HAILEY \\
\hline W & w & & & 19 & HAILEY \\
\hline 145 & 63 & 170 & 113 & 20 & HAllEY \\
\hline 81 & 27 & 171 & & 21 & HAILEY \\
\hline 109 & 43 & & & 22 & HAILEY \\
\hline $\mathrm{H}$ & $\mathrm{H}$ & & & 1 & HAILEY \\
\hline
\end{tabular}

$1: 63360$ or $1: 62500(15$-minute) or 1:24000 (7.5-minute) quadrangle

HOT SPRING 7.5

ROUGH MOUNTAIN 7.5 WRIGHT CREEK 7.5

HEISE 7.5

HEISE 7.5

DRIGGS 15

CONANT VALLEY 7.5

(ALPINE 7.5)

HERMAN 7.5

(CRANES FLAT 15)

YANDELL SPRINGS 15

YANDELL SPRINGS 15

ALTURAS LAKE 7.5 RYAN PEAK 7.5

(RYAN PEAK 7.5)

EASLEY HOT SPRINGS 7.5

EASLEY HOT SPRINGS 7.5

BAKER PEAK 7.5

GRIFFIN BUTTE 7.5

PARADISE PEAK 7.5

GRIFFIN BUTTE 75

BOARDMAN CREEK 7.5

SYDNEY BUTTE 7.5

SYDNEY BUTTE 7.5

MAHONEY BUTTE 7.5

HAILEY 7.5

BLAINE 15

FAIRFIELD 15

BELLEVUE 15

(FAIRFIELD 15)

(FAIRFIELD 15)

DAVIS MOUNTAIN 15 DAVIS MOUNTAIN 15

DAVIS MOUNTAIN 15)

ATLANTA WEST 7.5 


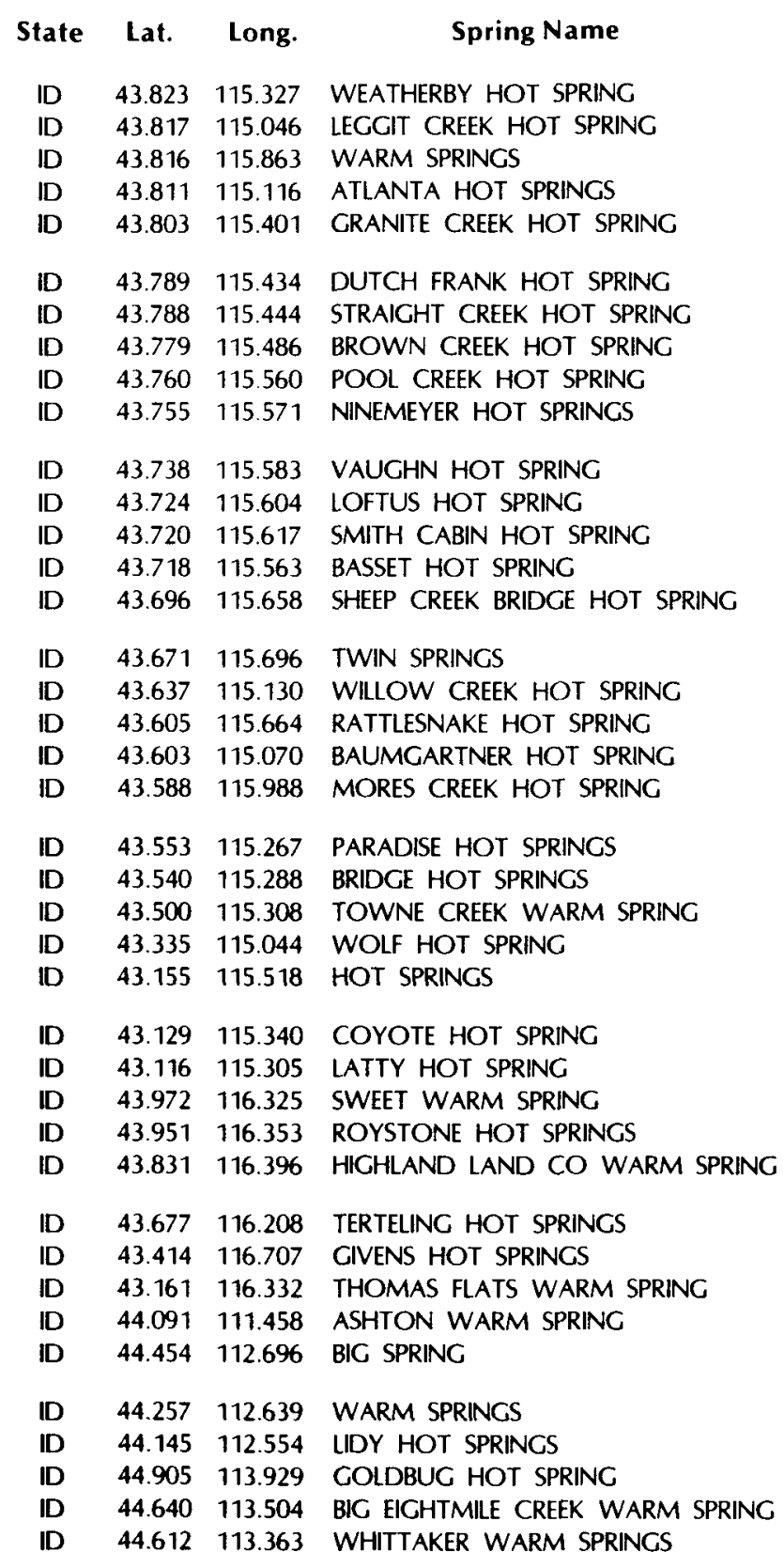

\begin{tabular}{|c|c|c|c|c|c|}
\hline \multicolumn{2}{|c|}{ Temperature } & $\begin{array}{l}\text { P.P. } \\
492\end{array}$ & $\begin{array}{c}\text { Circ. } \\
790\end{array}$ & NOAA & $1: 250,000($ AMS)map \\
\hline 113 & 45 & 122 & & 2 & HAllEY \\
\hline$H$ & $\mathrm{H}$ & & & 3 & HAllEY \\
\hline 108 & 42 & 82 & & 4 & HAILEY \\
\hline 140 & 60 & 123 & & 5 & HAILEY \\
\hline 131 & 55 & 120 & & 6 & HAILEY \\
\hline 149 & 65 & 119 & & 7 & HAILEY \\
\hline 144 & 62 & 118 & & 8 & HAILEY \\
\hline 122 & 50 & 117 & & 9 & HAILEY \\
\hline 108 & 42 & & & 10 & HAILEY \\
\hline 169 & 76 & 116 & 98 & 11 & HAILEY \\
\hline 154 & 68 & 115 & & 12 & HAILEY \\
\hline 129 & 54 & 113 & & 13 & HAILEY \\
\hline 138 & 59 & 112 & & 14 & HAIlEY \\
\hline $\mathrm{H}$ & $H$ & & & 15 & HAILEY \\
\hline 142 & 61 & 110 & & 16 & HAILEY \\
\hline 151 & 66 & 84 & & 17 & HAILEY \\
\hline 131 & 55 & 126 & & 18 & HAlLEY \\
\hline 133 & 56 & & & 19 & HAlLEY \\
\hline 111 & 44 & 127 & & 20 & HAILEY \\
\hline $\mathrm{H}$ & $\mathrm{H}$ & & & 21 & HAILEY \\
\hline 126 & 52 & 129 & & 22 & HAILEY \\
\hline 138 & 59 & 128 & & 23 & HAILEY \\
\hline 75 & 24 & & & 24 & HAllEY \\
\hline 113 & 45 & & & 25 & HAILEY \\
\hline 158 & 70 & 131 & & 26 & HAllEY \\
\hline 135 & 57 & & & 27 & HAILEY \\
\hline 144 & 62 & $131 \mathrm{~A}$ & 99 & 28 & HAILEY \\
\hline 68 & 20 & & & 1 & BOISE \\
\hline 151 & 66 & 66 & 95 & 2 & BOISE \\
\hline 73 & 23 & & & 3 & BOISE \\
\hline 106 & 41 & 68 & & 4 & BOISE \\
\hline 117 & 47 & 159 & & 5 & BOISE \\
\hline 75 & 24 & & & 6 & BOISE \\
\hline 79 & 26 & & 116 & 1 & ASHTON \\
\hline 73 & 23 & & & 1 & DUBOIS \\
\hline 84 & 29 & 148 & & 2 & DUBOIS \\
\hline 124 & 51 & 150 & & 3 & DUBOIS \\
\hline 113 & 45 & 61 & & 1 & DUBOIS \\
\hline 91 & 33 & & & 2 & DUBOIS \\
\hline 75 & 24 & 65 & & 3 & DUBOIS \\
\hline
\end{tabular}

1:63360 or 1:62500 (15-minute) or 1:24000 (7.5-minute) quadrangle

PHIFER CREEK 7.5

(ATLANTA EAST 7.5)

IDAHO CITY 7.5

ATLANTA EAST 7.5

GRAND MTN. 7.5

GRAND MTN 7.5

(CRAND MTN. 7.5)

(GRAND MTN. 7.5)

(BARBER FLAT 7.5)

BARBER FLAT 7.5

SHEEP CREEK 7.5

SHEEP CREEK 7.5

SHEEP CREEK 7.5

(SHEEP CREEK 7.5)

TWIN SPRINGS 7.5

TWIN SPRINGS 7.5

CAYUSE POINT 7.5

(LONG GULCH 7.5)

JUMBO MTN. 7.5

(ARROWROCK DAM 7.5)

FEATHERVILLE 7.5

(FEATHERVILLE 7.5)

(PINE 7.5)

(HILL CITY 7.5)

MOUNTAIN HOME 15

\section{(BENNETT MTN. 15)}

BENNETT MTN. 15

(MONTOUR 15)

MONTOUR 15

MONTOUR 15

BOISE NORTH 7.5

GIVENS HOT SPRINCS 7.5

WILD HORSE BUTTE 7.5

(ASHTON 7.5)

EDIE RANCH 15

\section{EDIE RANCH 15}

LIDY HOT SPRINGS 7.5

(GOLDBUC RIDGE 7.5)

(PATTERSON 15)

LEADORE 15 


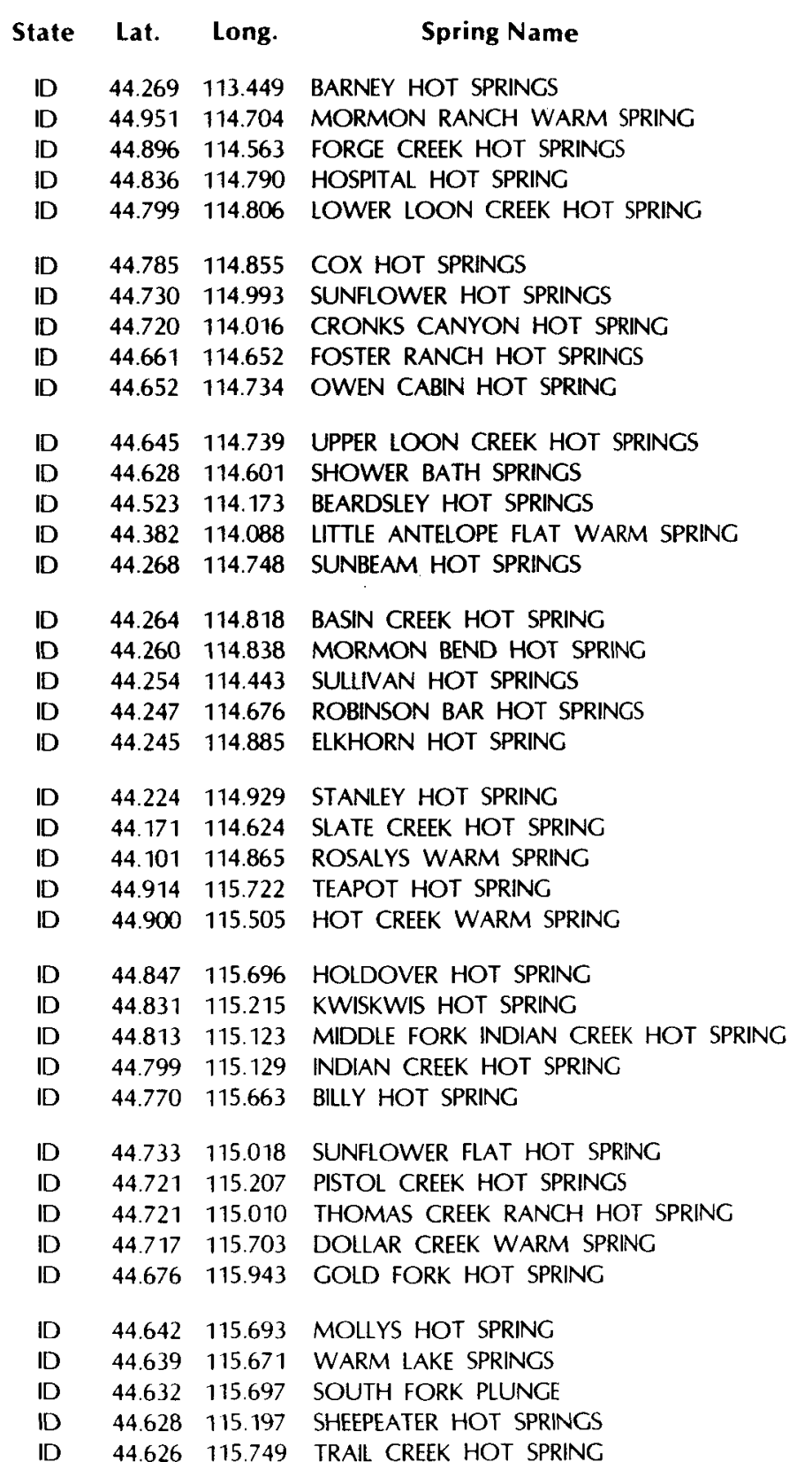

\section{P.P. Circ. NOAA 1:250,000(AMS)map 492790}

${ }^{\circ} \mathrm{F}{ }^{\circ} \mathrm{C}$

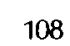

54

55

49

56

.

$\begin{array}{ll}4 & \text { DUBOIS } \\ 1 & \text { CHALLIS } \\ 2 & \text { CHALIS }\end{array}$

1.63360 or $1: 62500(15$-minute) or 1:24000 (7.5-minute) quadrangle

CILMORE 15

APAREJO POINT 7.5

YELLOWJACKET 7.5

RAMEY HILL 7.5

RAMEY HILL 7.5

RAMEY HILL 7.5

SLIDEROCK RIDCE 7.5

CHALLIS 15

ROCK CREEK 7.5

ROCK CREEK 7.5

ROCK CREEK 7.5

SHELDON PEAK 7.5

CHALLIS 15

LITTLE ANTELOPE FLAT 7.5

SUNBEAM 7.5

(EAST BASIN CREEK 7.5)

(EAST BASIN CREEK 7.5 )

CLAYTON 7.5

ROBINSON BAR 7.5

(STANLEY 7.5)

STANLEY 7.5

LIVINGSTON CREEK 7.5

OBSIDIAN 7.5

TEAPOT MTN. 7.5

CATON LAKE 7.5

WHITE ROCK PEAK 7.5

BIC BALDY 7.5

PUNGO MOUNTAIN 7.5

(BIC BALDY 7.5)

WHITE ROCK PEAK 7.5

(GREYHOUND RIDGE 15)

GREYHOUND RIDGE 15

CREYHOUND RIDGE

(WARM LAKE 15)

COID FORK 15

(WARM LAKE 15)

(WARM LAKE 15)

(WARM LAKE 15)

CREYHOUND RIDCE 15

(WARM LAKE 15) 


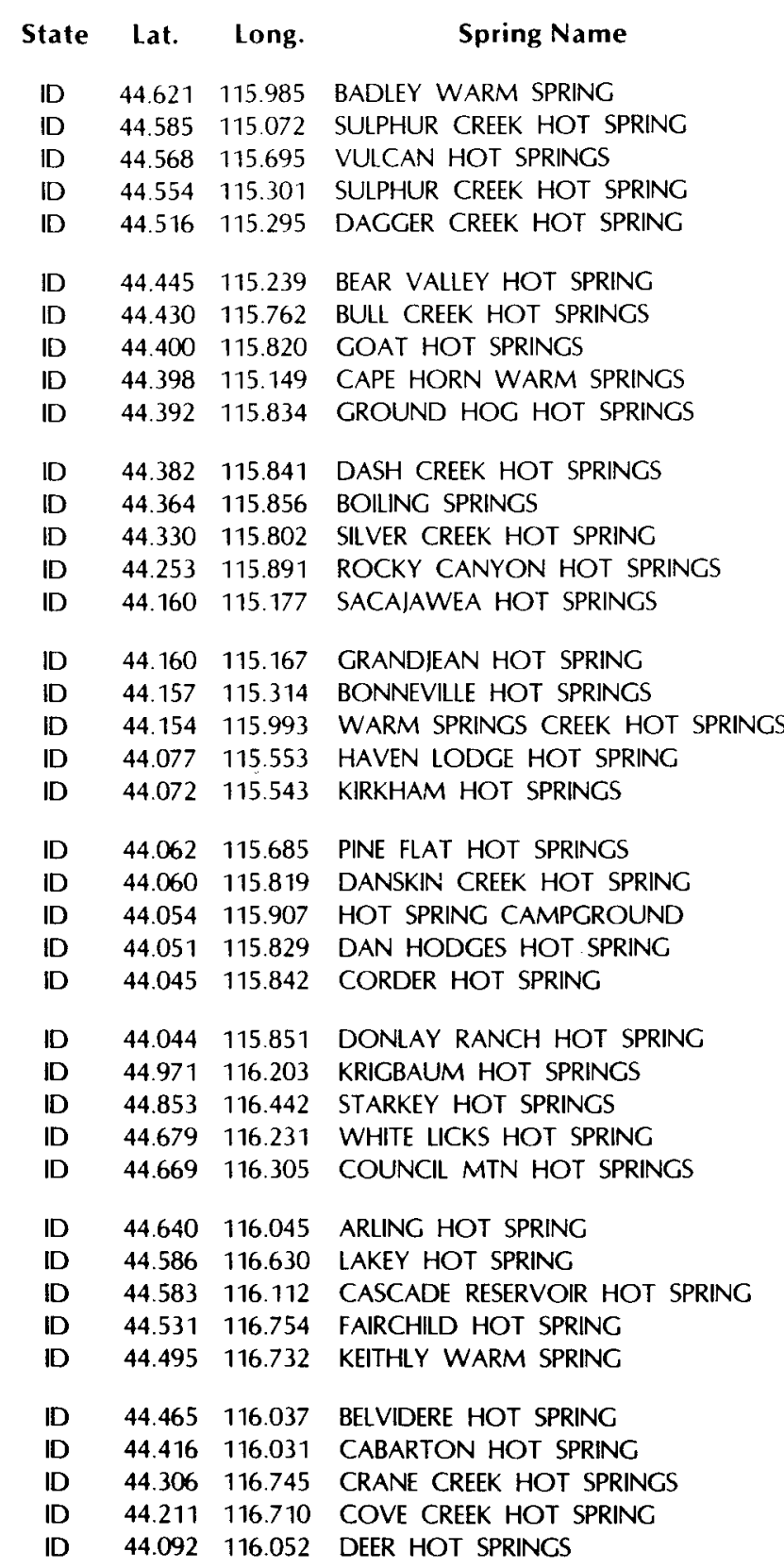

\begin{tabular}{|c|c|c|c|c|c|}
\hline \multicolumn{2}{|c|}{ Temperature } & \multirow{2}{*}{$\begin{array}{l}\text { P.P. } \\
492\end{array}$} & \multirow{2}{*}{$\begin{array}{c}\text { Circ. } \\
799\end{array}$} & \multirow[t]{2}{*}{ NOAA } & \multirow[t]{2}{*}{$1: 250,000(\mathrm{AMS}) \mathrm{map}$} \\
\hline${ }^{\circ} \mathbf{F}$ & ${ }^{\circ} \mathbf{C}$ & & & & \\
\hline 100 & 38 & & & 18 & CHALLIS \\
\hline $\mathrm{H}$ & $\mathrm{H}$ & & & 19 & CHALLIS \\
\hline 190 & 88 & 32 & & 20 & CHALLIS \\
\hline $\mathrm{H}$ & $\mathrm{H}$ & 43 & & 21 & CHALLIS \\
\hline 110 & 43 & 42 & & 22 & CHALLIS \\
\hline W & W & 41 & & 23 & CHALLIS \\
\hline $\mathrm{H}$ & $\mathrm{H}$ & 39 & & 24 & CHALLIS \\
\hline $\mathrm{H}$ & $H$ & 35 & & 25 & CHALLIS \\
\hline 95 & 35 & 87 & & 26 & CHALLIS \\
\hline 100 & 38 & 36 & & 27 & CHALLIS \\
\hline 138 & 59 & 37 & & 28 & CHALLIS \\
\hline 185 & 85 & 38 & & 29 & CHALLIS \\
\hline 102 & 39 & 40 & & 30 & CHALLIS \\
\hline 120 & 49 & & & 31 & CHALLIS \\
\hline 153 & 67 & 81 & & 32 & CHALLIS \\
\hline$H$ & $\mathrm{H}$ & & & 33 & CHALLIS \\
\hline 185 & 85 & 80 & 96 & 34 & CHALLIS \\
\hline 167 & 75 & 73 & & 35 & CHALLIS \\
\hline 147 & 64 & 78 & & 36 & CHALLIS \\
\hline 149 & 65 & 79 & & 37 & CHALLIS \\
\hline 138 & 59 & 77 & & 38 & CHALLIS \\
\hline 106 & 41 & & & 39 & CHALLIS \\
\hline 124 & 51 & 74 & & 40 & CHALLIS \\
\hline 140 & 60 & 75 & & 41 & CHALLIS \\
\hline 131 & 55 & 76 & & 42 & CHALLIS \\
\hline 131 & 55 & & & 43 & CHALLIS \\
\hline 109 & 43 & 17 & & 1 & BAKER \\
\hline 131 & 55 & & & 2 & BAKER \\
\hline 140 & 60 & 19 & & 3 & BAKER \\
\hline 154 & 68 & 18 & & 4 & BAKER \\
\hline 90 & 32 & 27 & & 5 & BAKER \\
\hline 158 & 70 & & & 6 & BAKER \\
\hline $\mathrm{H}$ & $H$ & & & 7 & BAKER \\
\hline 126 & 52 & & & 8 & BAKER \\
\hline 86 & 30 & & & 9 & BAKER \\
\hline 111 & 44 & & & 10 & BAKER \\
\hline 160 & 71 & & & 11 & BAKER \\
\hline 198 & 92 & & & 12 & BAKER \\
\hline 131 & 55 & & & 13 & BAKER \\
\hline 176 & 80 & & 97 & 14 & BAKER \\
\hline
\end{tabular}

1:63360 or 1:62500 (15-minute) or 1:24000 (7.5-minute) quadrangle

(COLD FORK 15)

(GREYHOUND RIDGE 15)

WARM LAKE 15

(CHINOOK MTN. 15)
(CHINOOK MTN. 15)

CAPE HORN LAKES 7.5

BOILING SPRINGS 15

BOILING SPRINCS 15

CAPE HORN LAKES 7.5

BOILING SPRINGS 15

BOILING SPRINCS 15

BOILING SPRINCS 15

BOILING SPRINGS 15

BOILING SPRINCS 15

GRANDJEAN 7.5

GRANDIEAN 7.5

EIGHTMILE MTN. 7.5

GARDEN VALLEY 15

(LOWMAN 7.5)

LOWMAN 7.5

PINE FLAT 7.5

GARDEN VALLEY 15

GARDEN VALLEY 15

(GARDEN VALLEY 15)

GARDEN VALLEY 15

(GARDEN VALLEY 15)

MEADOWS 7.5

NEW MEADOWS 15

CASCADE 15

COUNCIL 15

CASCADE 15

(CAMBRIDCE 15)

(CASCADE 15)

STURGILL PEAK 15

(CRANE CREEK RESERVOIR 15)

(SMITHS FERRY 15)

(SMITHS FERRY 15)

(CRANE CREEK RESERVOIR 15)

(HOLLAND GULCH 7.5)

BANKS 15 


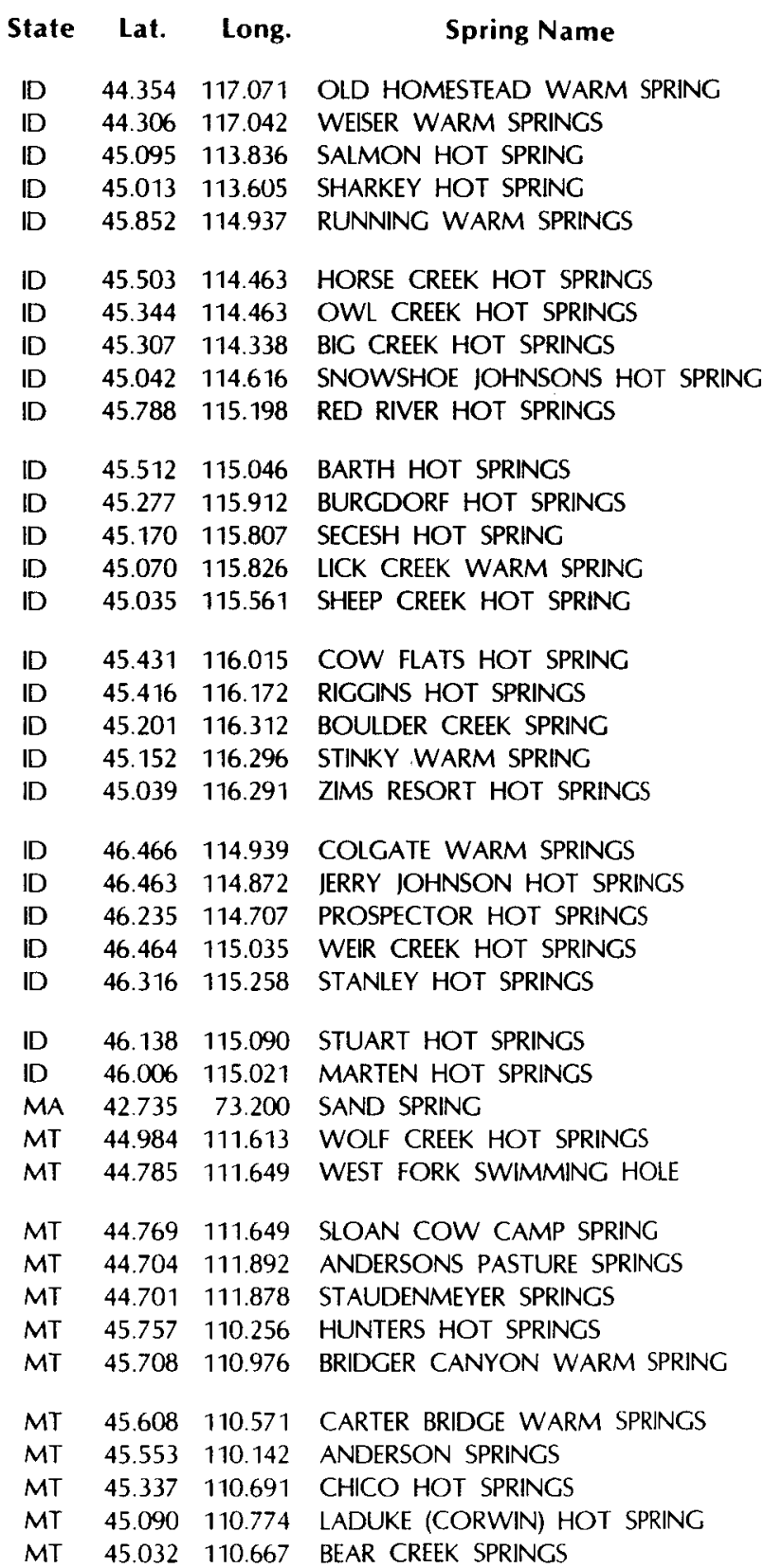

\begin{tabular}{|c|c|c|c|c|c|c|}
\hline \multicolumn{2}{|c|}{$\begin{array}{l}\text { Temperature } \\
{ }^{\circ} \mathrm{F}{ }^{\circ} \mathrm{C}\end{array}$} & $\begin{array}{l}\text { P.P. } \\
492\end{array}$ & $\begin{array}{l}\text { Circ. } \\
790\end{array}$ & \multicolumn{2}{|c|}{ NOAA 1:250,000(AMS)map } & $\begin{array}{l}1: 63360 \text { or } 1: 62500(15 \text {-minute }) \\
\text { or } 1: 24000 \text { ( } 7.5 \text {-minute) quadrangle }\end{array}$ \\
\hline W & W & & & 1 & BAKER & OLDS FERRY SE 7.5 \\
\hline 72 & 22 & & & 2 & BAKER & OLDS FERRY SE 7.5 \\
\hline 113 & 45 & 59 & & 1 & DILLON & SALMON 15 \\
\hline 126 & 52 & 60 & 106 & 2 & DILLON & GOLDSTONE MTN. 15 \\
\hline 106 & 41 & 9 & & 1 & ELK CITY & THREE PRONG MTN. 7.5 \\
\hline 109 & 43 & 50 & & 2 & ELK CITY & PAINTED ROCKS LAKE 15 \\
\hline 122 & 50 & 51 & 104 & 3 & ELK CITY & SHOUP 15 \\
\hline 199 & 93 & 52 & & 4 & ELK CITY & SHOUP 15 \\
\hline 108 & 42 & & & 5 & ELK CITY & (HOODOO MEADOWS 7.5) \\
\hline 131 & 55 & 10 & & 1 & ELK CITY & SABLE HILL 7.5 \\
\hline 140 & 60 & 11 & & 2 & ELK CITY & SHEEP HILL 7.5 \\
\hline 113 & 45 & 14 & & 3 & ELK CITY & BURCDORF 15 \\
\hline $\mathrm{H}$ & $\mathrm{H}$ & 22 & & 4 & ELK CITY & (LOON LAKE 7.5) \\
\hline 91 & 33 & 23 & & 5 & ELK CITY & (ENOS LAKE 7.5) \\
\hline 136 & 58 & 24 & & 6 & ELK CITY & (PARKS PEAK 7.5) \\
\hline 138 & 59 & 12 & & 1 & GRANCEVILLE & (KELLY MOUNTAIN 7.5) \\
\hline 106 & 41 & 13 & & 2 & GRANCEVILLE & RICGINS HOT SPRINCS 7.5 \\
\hline 82 & 28 & & & 3 & GRANCEVILLE & INDIAN MOUNTAIN 7.5 \\
\hline 88 & 31 & 15 & & 4 & GRANCEVILLE & INDIAN MOUNTAIN 7.5 \\
\hline 144 & 62 & 16 & & 5 & GRANGEVILLE & BALLY MOUNTAIN 7.5 \\
\hline 106 & 41 & 2 & & 1 & HAMILTON & BEAR MOUNTAIN 7.5 \\
\hline 118 & 48 & 3 & & 2 & HAMILTON & TOM BEAL PEAK 7.5 \\
\hline $\mathrm{H}$ & $\mathrm{H}$ & 7 & & 3 & HAMILTON & (WAHOO PEAK 7.5 ) \\
\hline 117 & 47 & 1 & & 1 & HAMILTON & GREYSTONE BUTTE 7.5 \\
\hline 120 & 49 & 5 & & 2 & HAMILTON & HUCKLEBERRY BUTTE 7.5 \\
\hline $\mathrm{H}$ & $\mathrm{H}$ & 6 & & 3 & HAMILTON & BIG ROCK MTN. 7.5 \\
\hline $\mathrm{H}$ & $\mathrm{H}$ & 8 & & 4 & HAMILTON & MINK PEAK 7.5 \\
\hline 75 & 24 & 1 & & 1 & ALBANY & WILLIAMSTOWN 7.5 \\
\hline 154 & 68 & & & 1 & ASHTON & CLIFF LAKE 15 \\
\hline 79 & 26 & & & 2 & ASHTON & (CLIFF LAKE 15) \\
\hline 86 & 30 & & & 3 & ASHTON & (CLIFF LAKE 15) \\
\hline 82 & 28 & & & 4 & ASHTON & LOWER RED ROCK LAKE 15 \\
\hline 82 & 28 & & & 5 & ASHTON & (LOWER RED ROCK LAKE 15) \\
\hline 140 & 60 & 36 & & 1 & BOZEMAN & HUNTERS HOT SPRINGS 7.5 \\
\hline 70 & 21 & & & 2 & BOZEMAN & (BOZEMAN PASS 15) \\
\hline 82 & 28 & & & 3 & BOZEMAN & (BRISBIN 7.5) \\
\hline 77 & 25 & 40 & & 4 & BOZEMAN & MCLEOD BASIN 7.5 \\
\hline 113 & 45 & 37 & & 5 & BOZEMAN & EMIGRANT 15 \\
\hline 154 & 68 & 38 & & 6 & BOZEMAN & MINER 15 \\
\hline 72 & 22 & 39 & & 7 & BOZEMAN & (GARDINER 15) \\
\hline
\end{tabular}




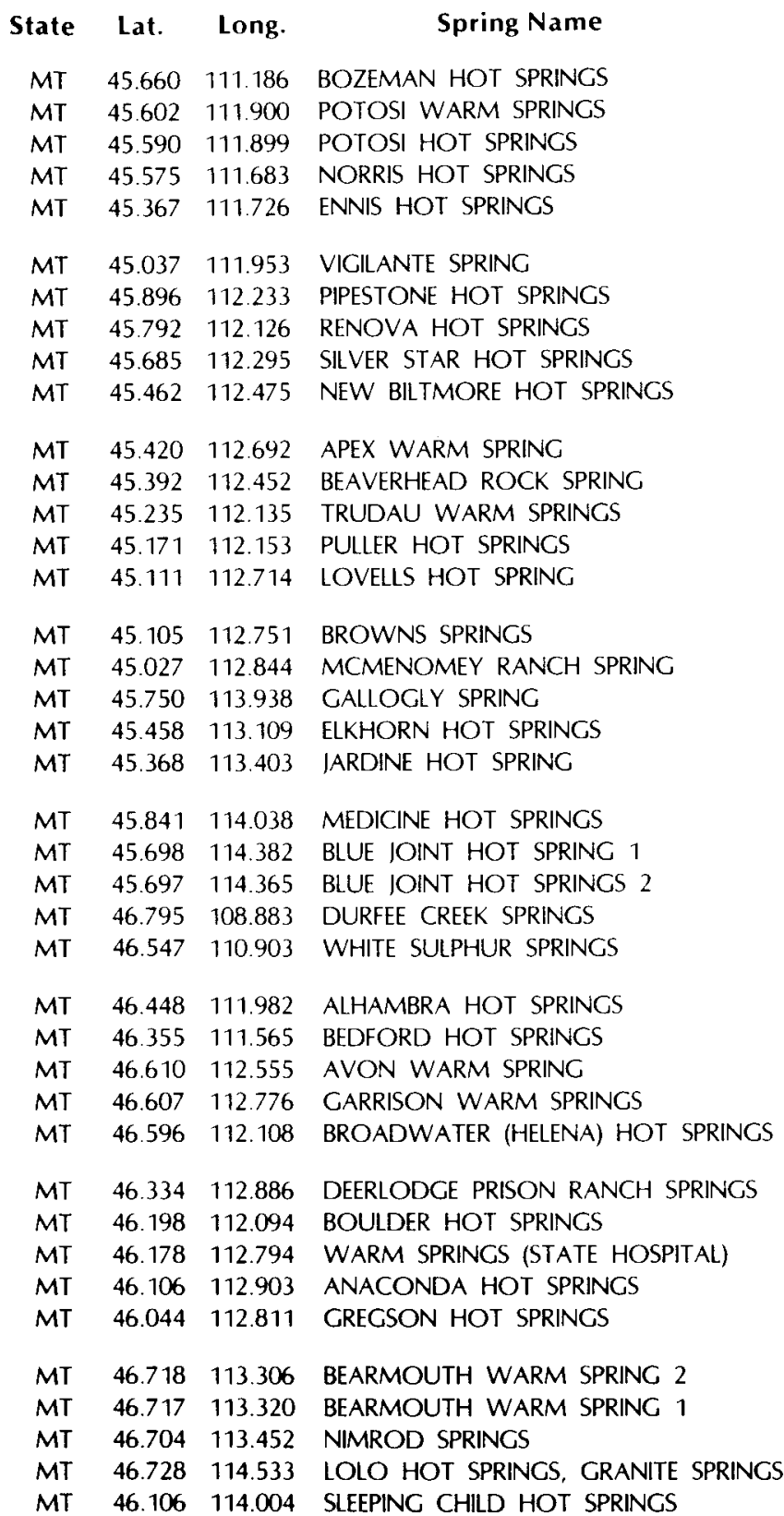

\begin{tabular}{|c|c|c|c|c|c|c|}
\hline Temp & ture & P.P. & Circ. & NOAA & $1: 250,000$ (AMS)map & $1: 63360$ or $1: 62500$ (15-minute) \\
\hline${ }^{\circ} \mathbf{F}$ & C & 492 & 790 & & & or 1:24000 (7.5-minute) quadrangle \\
\hline 131 & 55 & 35 & & 1 & BOZEMAN & BOZEMAN 15 \\
\hline 124 & 51 & & & 2 & BOZEMAN & HARRISON 15 \\
\hline 122 & 50 & 31 & & 3 & BOZEMAN & HARRISON 15 \\
\hline 127 & 53 & 32 & 127 & 4 & BOZEMAN & NORRIS 15 \\
\hline 181 & 83 & & 129 & 5 & BOZEMAN & (ENNIS 15) \\
\hline 75 & 24 & & & 6 & BOZEMAN & VARNEY 15 \\
\hline 135 & 57 & 20 & & 1 & DILLON & DRY MOUNTAIN 7.5 \\
\hline 122 & 50 & & & 2 & DILLON & (VENDOME 7.5) \\
\hline 163 & 73 & 30 & 128 & 3 & DILLON & (TWIN BRIDCES 15) \\
\hline 127 & 53 & & & 4 & DILLON & BEAVERHEAD ROCK 7.5 \\
\hline 77 & 25 & 27 & & 5 & DILLON & GLEN 7.5 \\
\hline 81 & 27 & & & 6 & DILLON & BEAVERHEAD ROCK 7.5 \\
\hline 73 & 23 & & & 7 & DILLON & METZEL RANCH 7.5 \\
\hline 111 & 44 & 33 & & 8 & DILLON & METZEL RANCH 7.5 \\
\hline 72 & 22 & 28 & & 9 & DILLON & GALLAGHER MOUNTAIN 7.5 \\
\hline 75 & 24 & 29 & & 10 & DILLON & DALYS 7.5 \\
\hline 68 & 20 & & & 11 & DILLON & DALYS 7.5 \\
\hline 100 & 38 & 14 & & 1 & DILLON & LOST TRAIL PASS 7.5 \\
\hline 118 & 48 & 26 & & 2 & DILLON & POLARIS 15 \\
\hline 140 & 60 & 25 & & 3 & DILLON & JACKSON 7.5 \\
\hline 117 & 47 & & & 1 & ELK CITY & MEDICINE HOT SPRINCS 7.5 \\
\hline 84 & 29 & & & 2 & ELK CITY & PAINTED ROCKS LAKE 15 \\
\hline 84 & 29 & 13 & & 3 & ELK CITY & PAINTED ROCKS LAKE 15 \\
\hline 70 & 21 & 11 & & 1 & ROUNDUP & \\
\hline 136 & 58 & 24 & & 1 & WHITE SULPHUR SPRINCS & WHITE SULPHUR SPRINGS 7.5 \\
\hline 138 & 59 & 18 & 124 & 1 & WHITE SULPHUR SPRINGS & (CLANCY 15) \\
\hline 74 & 24 & 21 & & 2 & WHITE SULPHUR SPRINGS & TOWNSEND 15 \\
\hline 79 & 26 & & & 1 & BUTTE & (AVON 15) \\
\hline 77 & 25 & 5 & & 2 & BUTTE & (GARRISON 15) \\
\hline 151 & 66 & 7 & 123 & 3 & BUTTE & (HELENA 15) \\
\hline 79 & 26 & & & 4 & BUTTE & (MOUNT POWELL 7.5) \\
\hline 169 & 76 & 19 & 125 & 5 & BUTTE & BOULDER 15 \\
\hline 172 & 78 & 15 & & 6 & BUTTE & ANACONDA NE 7.5 \\
\hline 72 & 22 & 16 & & 7 & BUTTE & ANACONDA 15 \\
\hline 158 & 70 & 17 & 126 & 8 & BUTTE & ANACONDA 15 \\
\hline 68 & 20 & & & 1 & BUTTE & (BEARMOUTH 15) \\
\hline 68 & 20 & & & 2 & BUTTE & BEARMOUTH 15 \\
\hline 70 & 21 & & & 3 & BUTTE & (BEARMOUTH 15) \\
\hline 111 & 44 & 4 & & 1 & HAMILTON & LOLO HOT SPRINCS 7.5 \\
\hline 122 & 50 & 12 & & 2 & HAMILTON & DEER MOUNTAIN 7.5 \\
\hline
\end{tabular}




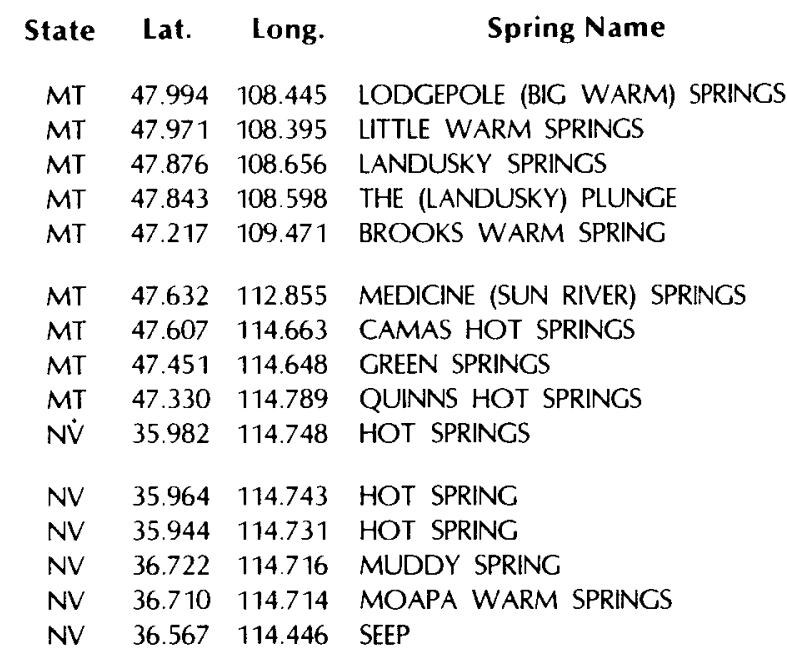

State Lat. Long.

Spring Name

MT $\quad 47.994108 .445$ LODGEPOLE (BIC WARM) SPRINGS

MT $\quad 47.971 \quad 108.395$ UTTLE WARM SPRINCS

MT $47876 \quad 108.656$ LANDUSKY SPRINCS

MT $\quad 47.843 \quad 108.598$ THE (LANDUSKY) PLUNG

MT $\quad 47.217 \quad 109.471$ BROOKS WARM SPRINC

MT $47.632 \quad 112.855$ MEDICINE (SUN RIVER) SPRINCS

MT $47.607 \quad 114.663$ CAMAS HOT SPRINGS

MT 47.451 114.648 GREEN SPRINCS

$\begin{array}{llll}\text { MT } & 47.330 & 114.789 & \text { QUINNS HOT SPRINGS }\end{array}$

$\begin{array}{llll}N \dot{ } & 35.982 & 114.748 & \text { HOT SPRINGS }\end{array}$

NV $\quad 35.964 \quad 114.743 \quad$ HOT SPRINC

NV $\quad 35.944 \quad 114.731 \quad$ HOT SPRINC

NV $\quad 36.722 \quad 114.716$ MUDDY SPRING

NV $\quad 36.710 \quad 114.714$ MOAPA WARM SPRINCS

NV $\quad 36.567 \quad 114.446$ SEEP

\begin{tabular}{|c|c|c|c|c|c|c|}
\hline \multicolumn{2}{|c|}{$\begin{array}{l}\text { Temperature } \\
{ }^{\circ} \mathrm{F} \quad{ }^{\circ} \mathrm{C}\end{array}$} & $\begin{array}{l}\text { P.P. } \\
492\end{array}$ & $\begin{array}{l}\text { Circ. } \\
790\end{array}$ & \multicolumn{2}{|c|}{ NOAA 1:250,000(AMS)map } & $\begin{array}{l}1: 63360 \text { or } 1: 62500(15 \text {-minute }) \\
\text { or } 1: 24000(7.5 \text {-minute }) \text { quadrangle }\end{array}$ \\
\hline 79 & 26 & 8 & & 1 & LEWISTOWN & (BEAR MOUNTAIN 7.5) \\
\hline 73 & 23 & 9 & & 2 & IEWISTOWN & (BEAR MOUNTAIN 7.5) \\
\hline 70 & 21 & & & 3 & LEWISTOWN & HAYS 7.5 \\
\hline 77 & 25 & & & 4 & LEWISTOWN & HAYS SE 7.5 \\
\hline 68 & 20 & 10 & & 1 & LEWISTOWN & LEWISTOWN 15 \\
\hline 86 & 30 & 6 & & 1 & CHOTEAU & ARSENIC PEAK 7.5 \\
\hline 113 & 45 & 1 & & 1 & WALLACE & HOT SPRINCS 7.5 \\
\hline 79 & 26 & & & 2 & WALLACE & PERMA 15 \\
\hline 109 & 43 & 3 & & 3 & WALLACE & PLAINS 15 \\
\hline 86 & 30 & & & 1 & KINGMAN & RINGBOLT RAPIDS 7.5 \\
\hline 82 & 28 & & & 2 & KINGMAN & RINGBOLT RAPIDS 7.5 \\
\hline 78 & 26 & & & 3 & KINGMAN & RINGBOLT RAPIDS 7.5 \\
\hline 90 & 32 & & & 1 & LAS VEGAS & MOAPA 15 \\
\hline 90 & 32 & 150 & & 2 & LAS VEGAS & MOAPA 15 \\
\hline 70 & 21 & & & 3 & LAS VEGAS & (OVERTON 15) \\
\hline 77 & 25 & & & 4 & LAS VEGAS & (OVERTON BEACH 15) \\
\hline 82 & 28 & & & 5 & LAS VEGAS & OVERTON BEACH 15 \\
\hline 82 & 28 & & & 6 & LAS VEGAS & OVERTON BEACH 15 \\
\hline 145 & 63 & & & 7 & LAS VEGAS & (HOOVER DAM 15) \\
\hline 72 & 22 & & & 1 & LAS VEGAS & (DOG BONE LAKE NORTH 7.5) \\
\hline 78 & 26 & 151 & & 2 & LAS VEGAS & INDIAN SPRINCS 7.5 \\
\hline 79 & 26 & & & 3 & LAS VEGAS & (INDIAN SPRINCS SE 7.5) \\
\hline 77 & 25 & 140 & & 4 & LAS VECAS & PAHRUMP 15 \\
\hline 75 & 24 & & & 5 & LAS VEGAS & LAS VEGAS NW 7.5 \\
\hline 78 & 26 & & & 6 & LAS VEGAS & LA MADRE MTN. 7.5 \\
\hline 79 & 26 & & & 7 & LAS VEGAS & (LAS VEGAS NW 7.5) \\
\hline 75 & 24 & 141 & & 8 & LAS VEGAS & PAHRUMP 15 \\
\hline 75 & 24 & & & 9 & LAS VEGAS & PAHRUMP 15 \\
\hline 72 & 22 & & & 1 & DEATH VALLEY & BARE MTN. 15 \\
\hline 109 & 43 & 138 & & 2 & DEATH VALLEY & BARE MTN. 15 \\
\hline 102 & 39 & & & 3 & DEATH VALLEY & BARE MTN. 15 \\
\hline 88 & 31 & & & 4 & DEATH VALLEY & BARE MTN. 15 \\
\hline 88 & 31 & & & 5 & DEATH VALLEY & BARE MTN. 15 \\
\hline 81 & 27 & & & 6 & DEATH VALLEY & ASH MEADOWS 15 \\
\hline 82 & 28 & & & 7 & DEATH VALLEY & ASH MEADOWS 15 \\
\hline 82 & 28 & 139 & & 8 & DEATH VALLEY & ASH MEADOWS 15 \\
\hline 93 & 34 & & & 9 & DEATH VALLEY & ASH MEADOWS 15 \\
\hline 81 & 27 & & & 10 & DEATH VALLEY & (ASH MEADOWS 15) \\
\hline 82 & 28 & & & 11 & DEATH VALLEY & (AMARGOSA FLAT 7.5) \\
\hline 86 & 30 & & & 12 & DEATH VALLEY & ASH MEADOWS 15 \\
\hline
\end{tabular}




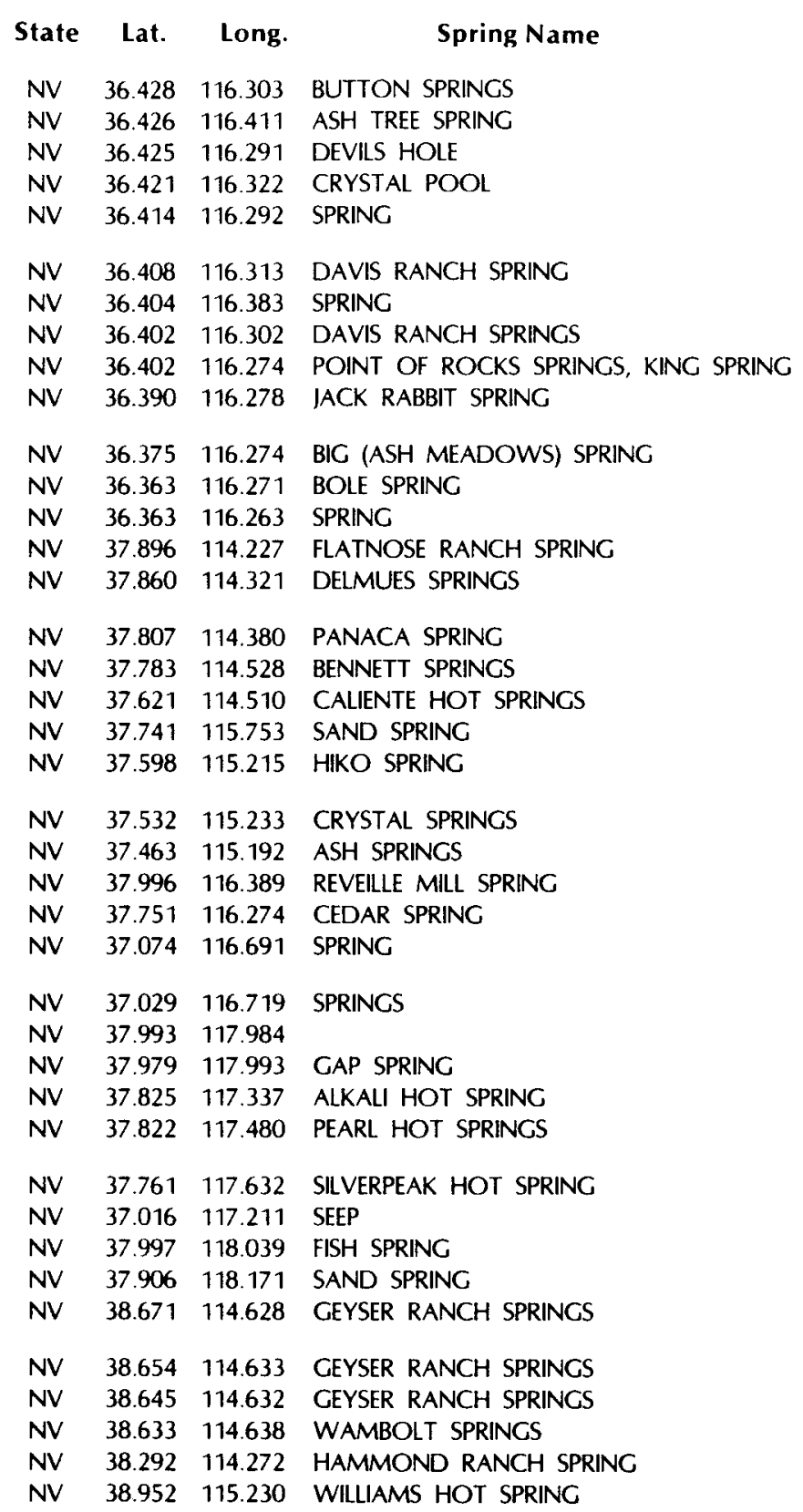

\begin{tabular}{|c|c|c|c|c|c|c|}
\hline \multicolumn{2}{|c|}{$\begin{array}{l}\text { Temperature } \\
{ }^{\circ} \mathbf{F}{ }^{\circ} \mathrm{C}\end{array}$} & $\begin{array}{l}\text { P.P. } \\
492\end{array}$ & $\begin{array}{l}\text { Circ. } \\
790\end{array}$ & \multicolumn{2}{|c|}{ NOAA 1:250,000(AMS)map } & $\begin{array}{l}1: 63360 \text { or } 1: 62500(15-\mathrm{mi} \\
\text { or } 1: 24000(7.5-\text { minute }) \mathrm{qu}\end{array}$ \\
\hline 93 & 34 & & & 13 & DEATH VALLEY & ASH MEADOWS 15 \\
\hline 75 & 24 & & & 14 & DEATH VALLEY & (ASH MEADOWS 15) \\
\hline 92 & 33 & & & 15 & DEATH VALLEY & ASH MEADOWS 15 \\
\hline 91 & 33 & & & 16 & DEATH VALLEY & ASH MEADOWS 15 \\
\hline 79 & 26 & & & 17 & DEATH VALLEY & (ASH MEADOWS 15) \\
\hline 70 & 21 & & & 18 & DEATH VALLEY & (ASH MEADOWS 15) \\
\hline 90 & 32 & & & 19 & DEATH VALLEY & (ASH MEADOWS 15) \\
\hline 81 & 27 & & & 20 & DEATH VALLEY & ASH MEADOWS 15 \\
\hline 91 & 33 & & & 21 & DEATH VALLEY & ASH MEADOWS 15 \\
\hline 82 & 28 & & & 22 & DEATH VALLEY & ASH MEADOWS 15 \\
\hline 83 & 28 & & & 23 & DEATH VALLEY & ASH MEADOWS 15 \\
\hline 72 & 22 & & & 24 & DEATH VALLEY & ASH MEADOWS 15 \\
\hline 72 & 22 & & & 25 & DEATH VALLEY & ASH MEADOWS 15 \\
\hline 77 & 25 & $144 B$ & & 1 & CALIENTE & URSINE 7.5 \\
\hline 70 & 21 & $144 \mathrm{~A}$ & & 2 & CALIENTE & CONDOR CANYON 7.5 \\
\hline 88 & 31 & 145 & & 3 & CALIENTE & PANACA 7.5 \\
\hline 70 & 21 & 144 & & 4 & CALIENTE & BENNETT PASS 7.5 \\
\hline 118 & 48 & 146 & & 5 & CALIENTE & (CALIENTE 7.5) \\
\hline 86 & 30 & & & 1 & CALIFNTE & WHITE BLOTCH SPRINCS 15 \\
\hline 90 & 32 & 147 & & 2 & CALIENTE & HIKO 7.5 \\
\hline 90 & 32 & 148 & & 3 & CALIENTE & HIKO 7.5 \\
\hline 97 & 36 & 149 & & 4 & CALIENTE & ASH SPRINGS 7.5 \\
\hline 84 & 29 & & & 1 & GOLDFIELD & (KAWICH PEAK 15) \\
\hline 77 & 25 & & & 2 & GOIDFIELD & KAWICH PEAK 15 \\
\hline 72 & 22 & & & 3 & GOLDFIELD & THIRSTY CANYON 15 \\
\hline 75 & 24 & & & 4 & GOLDFIELD & THIRSTY CANYON 15 \\
\hline 81 & 27 & & & 1 & GOLDFIELD & RHYOLITE RIDGE 15 \\
\hline 73 & 23 & & & 2 & GOLDFIELD & RHYOLITE RIDGE 15 \\
\hline 140 & 60 & 112 & & 3 & GOLDFIELD & ALKALI 7.5 \\
\hline 97 & 36 & & & 4 & GOLDFIELD & PAYMASTER RIDGE 7.5 \\
\hline 118 & 48 & 111 & & 5 & GOLDFIELD & SILVERPEAK 15 \\
\hline 77 & 25 & & & 6 & GOLDFIELD & BONNIE CLAIRE SW 7.5 \\
\hline 75 & 24 & & & 1 & MARIPOSA & (DAVIS MOUNTAIN 15) \\
\hline 74 & 23 & & & 2 & MARIPOSA & DAVIS MOUNTAIN 15 \\
\hline 70 & 21 & 142 & & 1 & LUND & MT. GRAFTON 7.5 \\
\hline 70 & 21 & 142 & & 2 & LUND & MT. GRAFTON 7.5 \\
\hline 70 & 21 & 142 & & 3 & LUND & MT. GRAFTON 7.5 \\
\hline 70 & 21 & 142 & & 4 & LUND & MT. GRAFTON 7.5 \\
\hline 84 & 29 & 143 & & 5 & LUND & (TRAIL CANYON 7.5) \\
\hline 127 & 53 & $103 \mathrm{~A}$ & & 1 & LUND & \\
\hline
\end{tabular}




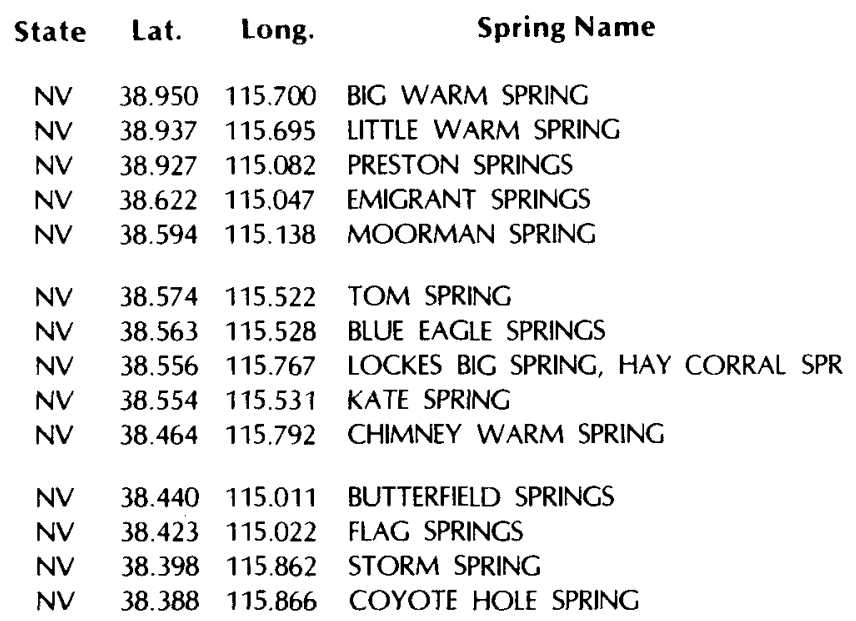

\begin{tabular}{|c|c|c|c|c|c|}
\hline Temp & iture & P.P. & Circ. & NOAA & $1: 250,000$ (AMS)map \\
\hline & & & & & \\
\hline 93 & 34 & 122 & & 2 & LUND \\
\hline 90 & 32 & & & 3 & IUND \\
\hline 70 & 21 & 104 & & 4 & LUND \\
\hline 70 & 21 & 135 & & 5 & LUND \\
\hline 100 & 38 & 134 & & 6 & LUND \\
\hline 72 & 22 & & & 7 & LUND \\
\hline 84 & 29 & 128 & & 8 & LUND \\
\hline 100 & 38 & 126 & & 9 & LUND \\
\hline 73 & 23 & 129 & & 10 & LUND \\
\hline 160 & 71 & 127 & & 11 & LUND \\
\hline 75 & 24 & 136 & & 12 & LUND \\
\hline 75 & 24 & & & 13 & LUND \\
\hline 99 & 37 & & & 14 & LUND \\
\hline 113 & 45 & & & 15 & LUND \\
\hline 90 & 32 & 137 & & 16 & LUND \\
\hline 115 & 46 & & & 17 & LUND \\
\hline 92 & 33 & $134 \mathrm{~A}$ & & 18 & LUND \\
\hline $\mathrm{H}$ & $\mathrm{H}$ & & & 1 & TONOPAH \\
\hline 95 & 35 & & & 2 & TONOPAH \\
\hline$w$ & W & 121 & & 3 & TONOPAH \\
\hline 106 & 41 & & & 4 & TONOPAH \\
\hline 95 & 35 & & & 5 & TONOPAH \\
\hline 102 & 39 & & & 6 & TONOPAH \\
\hline 180 & 82 & & & 7 & TONOPAH \\
\hline 142 & 61 & 124 & & 8 & TONOPAH \\
\hline w & W & & & 9 & TONOPAH \\
\hline 86 & 30 & & & 10 & TONOPAH \\
\hline 145 & 63 & 125 & & 11 & TONOPAH \\
\hline 86 & 30 & & & 12 & TONOPAH \\
\hline 80 & 27 & 116 & & 1 & TONOPAH \\
\hline 90 & 32 & & & 2 & TONOPAH \\
\hline 203 & 95 & 118 & 161 & 3 & TONOPAH \\
\hline W & W & 123 & & 4 & TONOPAH \\
\hline W & W & 108 & & 1 & WALKER LAKE \\
\hline 144 & 62 & 113 & & 2 & WALKER LAKE \\
\hline 110 & 43 & 109 & & 3 & WALKER LAKE \\
\hline 100 & 38 & 110 & & 4 & WALKER LAKE \\
\hline 160 & 71 & 60 & & 1 & WALKER LAKE \\
\hline W & $w$ & & & 2 & WALKER LAKE \\
\hline 144 & 62 & 61 & & 3 & WALKER LAKE \\
\hline
\end{tabular}

$1: 63360$ or $1: 62500$ (15-minute) or $1: 24000$ (7.5-minute) quadrangle

\section{DUCKWATER 15 \\ DUCKWATER 15}

MOORMAN SPRING SE 7.5 MOORMAN SPRING 7.5

BLUE EAGLE SPRINCS 15 BLUE EAGLE SPRINCS 15 LOCKES 7.5

THE WALL NE 7.5

SUNNYSIDE 7.5

SUNNYSIDE 7.5

THE WALL NE 7.5

THE WALL NE 7.5

SUNNYSIDE NW 7.5

THE WALL SE 7.5 HOT CREEK BUTTE 7.5 (MOSQUITO CREEK 7.5) MOSOUITO CREEK 7.5

FISH SPRINGS 7.5

UPPER FISH LAKE 7.5 UTTIE FISH LAKE 7.5 LITTLE FISH LAKE 7.5 HOBBLE CANYON 7.5 (BLUE IAY SPRING 7.5)

GEORGES CANYON RIM 7.5 SAULSBURY BASIN 7.5

WARM SPRINGS 7.5

CARVERS NE 7.5

CARVERS NE 7.5

CARVERS 7.5

(SAN ANTONIO RANCH 15)

GILLIS CANYON 15

(AURORA 15)

SODAVILLE 75

MINDEN 7.5

(WELLINGTON 15)

WEUINGTON 15 


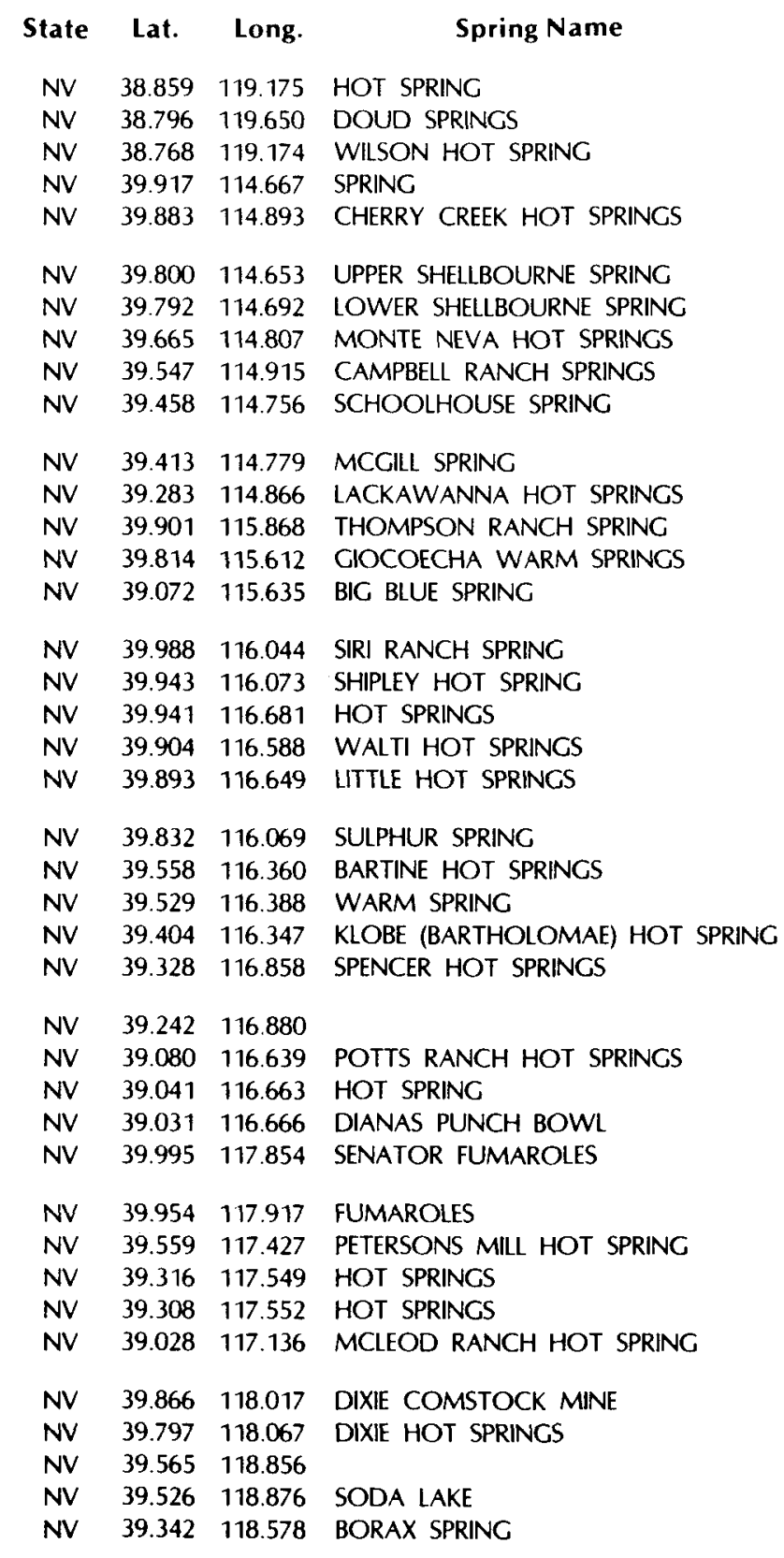

\begin{tabular}{|c|c|c|c|c|c|}
\hline Temp & ture & P.P. & Circ. & NOAA & $1: 250,000($ AMS)map \\
\hline $\mathrm{H}$ & $\mathrm{H}$ & & & 4 & WALKER LAKE \\
\hline 70 & 21 & & & 5 & WALKER LAKE \\
\hline 183 & 84 & & & 6 & WAIKER LAKE \\
\hline 83 & 28 & & & 1 & ELY \\
\hline 144 & 62 & 95 & 170 & 2 & ELY \\
\hline 74 & 23 & & & 3 & ELY \\
\hline 77 & 25 & & & 4 & ELY \\
\hline 176 & 80 & 98 & & 5 & ELY \\
\hline 76 & 24 & 100 & & 6 & ELY \\
\hline 84 & 29 & & & 7 & ELY \\
\hline 84 & 29 & 101 & & 8 & ELY \\
\hline 95 & 35 & 102 & & 9 & ELY \\
\hline 68 & 20 & 910 & & 1 & ELY \\
\hline 76 & 24 & $102 \mathrm{~A}$ & & 2 & ELY \\
\hline w & W & & & 3 & ELY \\
\hline 95 & 35 & $91 \mathrm{~A}$ & & 1 & MILLETT \\
\hline 106 & 41 & $91 \mathrm{~B}$ & & 2 & MILLETT \\
\hline 180 & 82 & 92 & & 3 & MILLETT \\
\hline 162 & 72 & 93 & & 4 & MILLETT \\
\hline$H$ & $H$ & & & 5 & MILLETT \\
\hline 74 & 23 & $91 \mathrm{C}$ & & 6 & MIILETT \\
\hline 108 & 42 & $93 \mathrm{~A}$ & & 7 & MHLETT \\
\hline w & W & & & 8 & MILLETT \\
\hline 156 & 69 & $93 \mathrm{~B}$ & & 9 & MILLETT \\
\hline 162 & 72 & 86 & 160 & 10 & MILLETT \\
\hline $\mathrm{H}$ & $\mathrm{H}$ & 87 & & 11 & MILLETT \\
\hline 113 & 45 & 119 & & 12 & MILLETT \\
\hline 124 & 51 & & & 13 & MILLETT \\
\hline 138 & 59 & & & 14 & MILLETT \\
\hline B & B & & & 1 & MILLETT \\
\hline 204 & 96 & 70 & & 2 & MIILLETT \\
\hline H & $\mathrm{H}$ & & & 3 & MILLETT \\
\hline 198 & 92 & 85 & 159 & 4 & MILLETT \\
\hline 115 & 46 & & 159 & 5 & MILLETT \\
\hline 180 & 82 & 114 & & 6 & MILLETT \\
\hline H & $\mathrm{H}$ & & & 1 & RENO \\
\hline 162 & 72 & $71 \mathrm{~A}$ & 149 & 2 & RENO \\
\hline 210 & 99 & & 144 & 3 & RENO \\
\hline 86 & 30 & & & 4 & RENO \\
\hline 180 & 82 & 74 & & 5 & RENO \\
\hline
\end{tabular}

1:63360 or 1:62500 (15-minute) or 1:24000 (7.5-minute) quadrangle

YERINGTON 15

MT. SIECEL 15

YERINGTON 15

MCGILL 7.5

MCGILL 7.5

EAST ELY 7.5

DIAMOND SPRINGS 15

COLD CREEK RANCH 15

GREEN SPRINGS 15

GARDEN VALLEY 15

GARDEN VALLEY 15

WALTI HOT SPRINGS 15

WALTI HOT SPRINGS 15

WALTI HOT SPRINGS 15

GARDEN VALLEY 15

BARTINE RANCH 15

BARTINE RANCH 15

ANTELOPE PEAK 15

SPENCER HOT SPRINCS 15

(WILDCAT PEAK 15)

DIANAS PUNCH BOWL 15

DIANAS PUNCH BOWL 15

DIANAS PUNCH BOWL 15

(HUMBOLDT SALT MARSH 15)

(HUMBOLDT SALT MARSH 15)

MOUNT AIRY 7.5

CARROLL SUMMIT SE 7.5

CARROLL SUMMIT SE 7.5

MILLETT RANCH 15

DIXIE HOT SPRINCS 15 DIXIE HOT SPRINCS 15

SODA LAKE 15

(SODA LAKE 15)

(CARSON LAKE 15) 


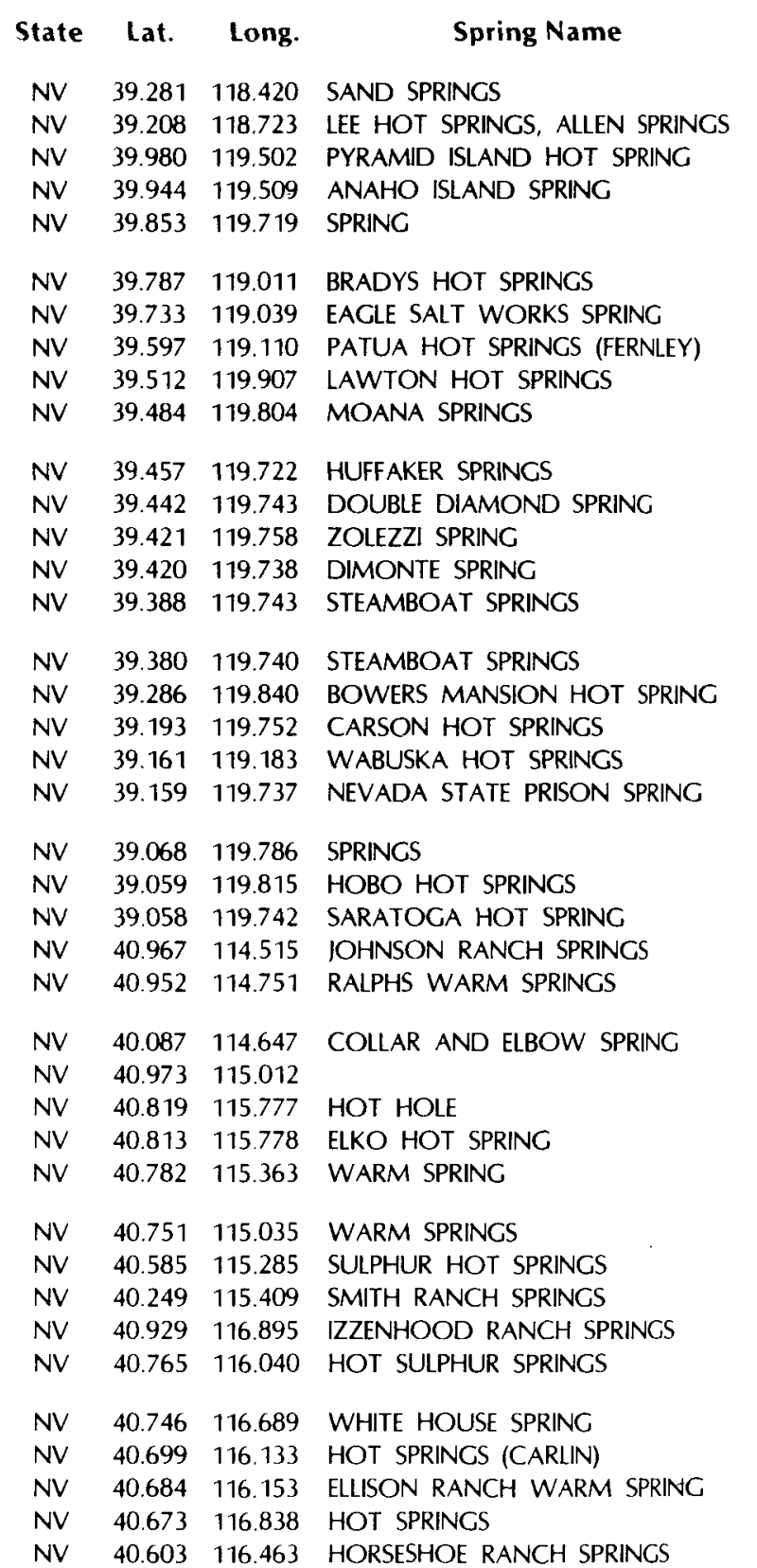

\begin{tabular}{|c|c|c|c|c|c|c|}
\hline \multicolumn{2}{|c|}{ Temperature } & \multirow{2}{*}{$\begin{array}{r}\text { P.P. } \\
\mathbf{4 9 2} \\
75\end{array}$} & $\begin{array}{l}\text { Circ. } \\
790\end{array}$ & \multicolumn{2}{|c|}{ NOAA 1:250,000(AMS)map } & $\begin{array}{l}1: 63360 \text { or } 1: 62500 \text { (15-minute) } \\
\text { or } 1: 24000(7.5 \text {-minute) quadrangle }\end{array}$ \\
\hline $\mathrm{H}$ & $\mathrm{H}$ & & & 6 & RENO & FOURMILE FLAT 7.5 \\
\hline 190 & 88 & $74 \mathrm{~A}$ & 143 & 7 & RENO & ALLEN SPRINGS 15 \\
\hline & & & & 1 & RENO & (SUTCLIFFE 15) \\
\hline 120 & 49 & 52 & & 2 & RENO & (SUTCLIFFE 15) \\
\hline 73 & 23 & 53 & & 3 & RENO & SUTCLIFFE 15 \\
\hline 209 & 98 & 72 & 147 & 4 & RENO & FIREBALL RIDCE 15 \\
\hline H & $\mathrm{H}$ & 73 & & 5 & RENO & (TWO TIPS 15) \\
\hline 187 & 86 & & 146 & 6 & RENO & (TWO TIPS 15) \\
\hline 120 & 49 & & & 7 & RENO & VERDI 7.5 \\
\hline 199 & 93 & $55 A$ & 140 & 8 & RENO & MT. ROSE NE 7.5 \\
\hline 81 & 27 & $55 B$ & & 9 & RENO & (STEAMBOAT 7.5) \\
\hline 84 & 29 & & & 10 & RENO & (STEAMBOAT 7.5 ) \\
\hline 102 & 39 & $55 \mathrm{C}$ & & 11 & RENO & (MT. ROSE NE 7.5) \\
\hline 127 & 53 & 55D & & 12 & RENO & (STEAMBOAT 7.5) \\
\hline 205 & 96 & & 141 & 13 & RENO & STEAMBOAT 7.5 \\
\hline 205 & 96 & 56 & 141 & 14 & RENO & STEAMBOAT 7.5 \\
\hline 133 & 56 & 57 & & 15 & RENO & WASHOE CITY 7.5 \\
\hline 120 & 49 & 59 & & 16 & RENO & CARSON CITY 7.5 \\
\hline 201 & 94 & 62 & 142 & 17 & RENO & WABUSKA 15 \\
\hline 75 & 24 & $59 A$ & & 18 & RENO & (NEW EMPIRE 7.5) \\
\hline 90 & 32 & & & 19 & RENO & GENOA 7.5 \\
\hline 114 & 46 & & & 20 & RENO & GENOA 7.5 \\
\hline 122 & 50 & & & 21 & RENO & MC TARNAHAN HILL 7.5 \\
\hline 72 & 22 & $30 \mathrm{C}$ & & 1 & ELKO & INDEPENDENCE VALLEY NE 7.5 \\
\hline 86 & 30 & & & 2 & ELKO & SNOW WATER LAKE NE 7.5 \\
\hline 93 & 34 & 94 & & 3 & ELKO & \\
\hline W & W & & & 1 & ELKO & (HUMBOLDT PEAK 7.5) \\
\hline 133 & 56 & & 166 & 2 & ELKO & ELKO WEST 7.5 \\
\hline 190 & 88 & 32 & 166 & 3 & ELKO & ELKO WEST 7.5 \\
\hline W & $w$ & 33 & & 4 & ELKO & SOLDIER PEAK 7.5 \\
\hline w & w & 34 & & 5 & ELKO & GORDON CREEK 7.5 \\
\hline 205 & 96 & & 169 & 6 & ELKO & LAMOILLE 15 \\
\hline 149 & 65 & $34 \mathrm{~A}$ & & 7 & ELKO & RUBY LAKE NW 7.5 \\
\hline 88 & 31 & 76 & & 1 & WINNEMUCCA & IZZENHOOD RANCH 7.5 \\
\hline 147 & 64 & 31 & & 2 & WINNEMUCCA & HUNTSMAN RANCH 7.5 \\
\hline W & w & 77 & & 3 & WINNEMUCCA & DUNPHY 15 \\
\hline 174 & 79 & & 165 & 4 & WINNEMUCCA & (CARLIN 15) \\
\hline W & w & & & 5 & WINNEMUCCA & CARLIN 15 \\
\hline 122 & 50 & & & 6 & WINNEMUCCA & BATTLE MOUNTAIN 15 \\
\hline 136 & 58 & 88 & & 7 & WINNEMUCCA & (BEOWAWE 15) \\
\hline
\end{tabular}




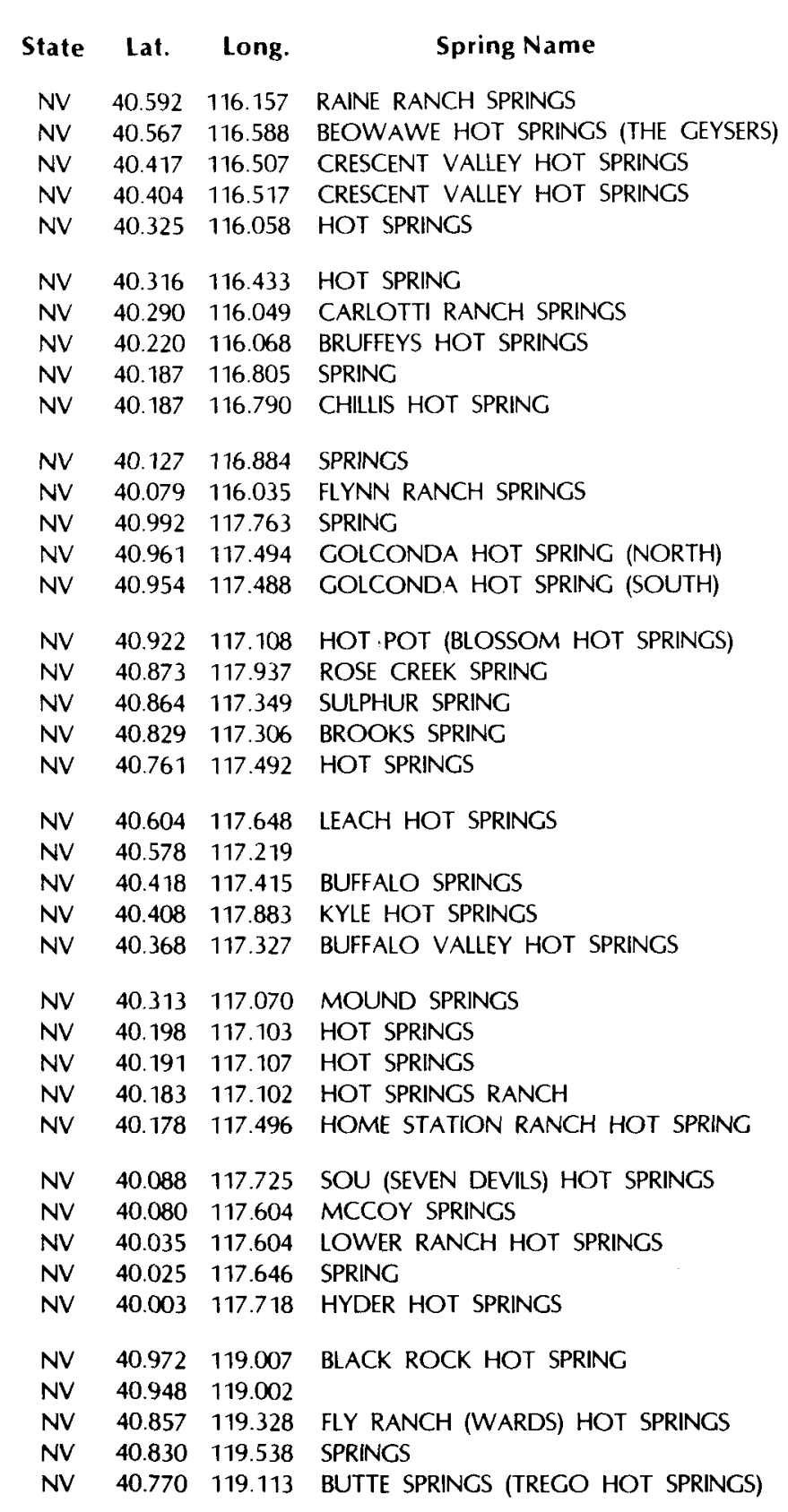

\begin{tabular}{|c|c|c|c|c|c|}
\hline \multicolumn{2}{|c|}{$\begin{array}{l}\text { Temperature } \\
{ }^{\circ} \mathrm{F}{ }^{\circ} \mathrm{C}\end{array}$} & \multirow[t]{2}{*}{$\begin{array}{l}\text { P.P. } \\
492\end{array}$} & \multirow[t]{2}{*}{$\begin{array}{c}\text { Circ. } \\
790\end{array}$} & NOAA & $1: 250,000($ AMS)map \\
\hline w & $w$ & & & 8 & WINNEMUCCA \\
\hline 208 & 98 & $77 \mathrm{~A}$ & 162 & 9 & WINNEMUCCA \\
\hline 138 & 59 & & 163 & 10 & WINNEMUCCA \\
\hline 138 & 59 & $88 \mathrm{~A}$ & 163 & 11 & WINNEMUCCA \\
\hline 79 & 26 & 89 & & 12 & WINNEMUCCA \\
\hline 186 & 86 & & & 13 & WINNEMUCCA \\
\hline 102 & 39 & 90 & & 14 & WINNEMUCCA \\
\hline 149 & 65 & $90 A$ & & 15 & WINNEMUCCA \\
\hline 72 & 22 & & & 16 & WINNEMUCCA \\
\hline 102 & 39 & 82 & & 17 & WINNEMUCCA \\
\hline 72 & 22 & & & 18 & WINNEMUCCA \\
\hline 78 & 26 & 91 & & 19 & WINNEMUCCA \\
\hline 93 & 34 & 18 & & 1 & WINNEMUCCA \\
\hline 165 & 74 & 19 & 155 & 2 & WINNEMUCCA \\
\hline 151 & 66 & 19 & 155 & 3 & WINNEMUCCA \\
\hline 136 & 58 & $19 \mathrm{~A}$ & 156 & 4 & WINNEMUCCA \\
\hline 83 & 28 & & & 5 & WINNEMUCCA \\
\hline $\mathrm{H}$ & $\mathrm{H}$ & & & 6 & WINNEMUCCA \\
\hline 93 & 34 & $19 \mathrm{~F}$ & & 7 & WINNEMUCCA \\
\hline 185 & 85 & $19 \mathrm{C}$ & 157 & 8 & WINNEMUCCA \\
\hline 207 & 97 & 64 & 154 & 9 & WINNEMUCCA \\
\hline 162 & 72 & & & 10 & WINNEMUCCA \\
\hline 162 & 72 & & & 11 & WINNEMUCCA \\
\hline 171 & 77 & 66 & 152 & 12 & WINNEMUCCA \\
\hline 174 & 79 & 78 & 158 & 13 & WINNEMUCCA \\
\hline 109 & 43 & 79 & & 14 & WINNEMUCCA \\
\hline 127 & 53 & 80 & & 15 & WINNEMUCCA \\
\hline 127 & 53 & & & 16 & WINNEMUCCA \\
\hline 129 & 54 & 81 & & 17 & WINNEMUCCA \\
\hline 135 & 57 & & & 18 & WINNEMUCCA \\
\hline 185 & 85 & 68 & 153 & 19 & WINNEMUCCA \\
\hline 120 & 49 & & & 20 & WINNEMUCCA \\
\hline 104 & 40 & & & 21 & WINNEMUCCA \\
\hline 83 & 28 & & & 22 & WINNEMUCCA \\
\hline 176 & 80 & 69 & & 23 & WINNEMUCCA \\
\hline 136 & 58 & 16 & 134 & 1 & LOVELOCK \\
\hline 194 & 90 & & 134 & 2 & LOVELOCK \\
\hline 180 & 82 & 37 & 136 & 3 & LOVELOCK \\
\hline 84 & 29 & & & 4 & LOVELOCK \\
\hline 187 & 86 & 63 & 135 & 5 & LOVELOCK \\
\hline
\end{tabular}

$1: 63360$ or $1: 62500$ ( 15 -minute) or 1:24000 (7.5-minute) quadrangle

CARLIN 15

DUNPHY 15

CRESCENT VALLEY 15 CRESCENT VALLEY 15 PINE VALLEY 15

FRENCHIE CREEK 15 PINE VALLEY 15

MINERAL HILL 15
CARICO LAKE 15

CARICO LAKE 15

CARICO LAKE 15 MINERAL HILL 15 ROSE CREEK 15 COLCONDA 7.5 GOLCONDA 7.5

HOT POT 7.5 ROSE CREEK 15 BROOKS SPRING 7.5 BROOKS SPRING 7.5 GOLDRUN CREEK 7.5

LEACH HOT SPRINCS 15 (ANTLER PEAK 15) BUFFALO SPRINCS 15 KYLE HOT SPRINGS 15 BUFFALO SPRINCS 15

MC COY 15 THE CEDARS 15 THE CEDARS 15 THE CEDARS 15 MT. MOSES 15

CAIN MOUNTAIN 15 CAIN MOUNTAIN 15 CAIN MOUNTAIN 15 CAIN MOUNTAIN 15 CAIN MOUNTAIN 15 


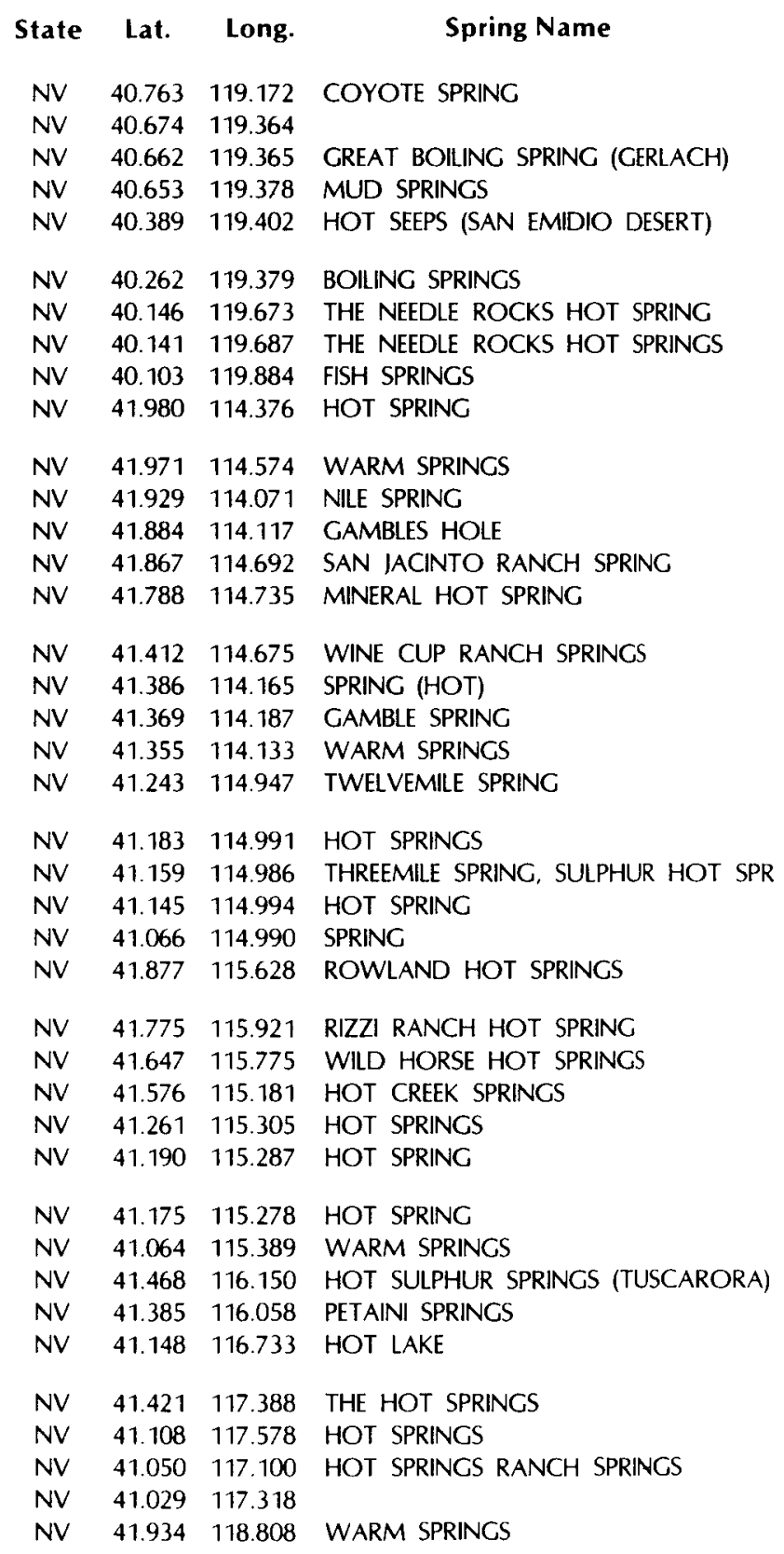

\begin{tabular}{|c|c|c|c|c|c|c|}
\hline \multicolumn{2}{|c|}{ Temperature } & $\begin{array}{l}\text { P.P. } \\
492\end{array}$ & $\begin{array}{c}\text { Circ. } \\
790\end{array}$ & NOAA & $1: 250,000($ AMS)map & $\begin{array}{l}1: 63360 \text { or } 1: 62500(15 \text {-minute }) \\
\text { or } 1: 24000(7.5 \text {-minute) quadrangle }\end{array}$ \\
\hline 72 & 22 & & & 6 & LOVELOCK & \\
\hline 194 & 90 & & 137 & 7 & LOVELOCK & (GERLACH 15) \\
\hline 208 & 98 & 38 & 137 & 8 & LOVELOCK & GERLACH 15 \\
\hline 184 & 84 & 39 & 137 & 9 & LOVELOCK & (GERLACH 15) \\
\hline 203 & 95 & & 138 & 10 & LOVELOCK & (KUMIVA PEAK 15) \\
\hline 187 & 86 & 50 & & 11 & LOVELOCK & KUMIVA PEAK 15 \\
\hline 208 & 98 & 49 & 139 & 12 & LOVELOCK & THE NEEDLE ROCKS 7.5 \\
\hline 208 & 98 & 49 & 139 & 13 & LOVELOCK & THE NEEDLE ROCKS 7.5 \\
\hline 73 & 23 & 48 & & 14 & LOVELOCK & STATE LINE PEAK 7.5 \\
\hline 86 & 30 & & & 1 & WELLS & COLLAHER MOUNTAIN 15 \\
\hline 95 & 35 & & & 2 & WELLS & DELAPLAIN 15 \\
\hline 109 & 43 & 24 & & 3 & WELLS & COOSE CREEK 15 \\
\hline 104 & 40 & 25 & & 4 & WELLS & COOSE CREEK 15 \\
\hline 148 & 64 & $22 \mathrm{~B}$ & & 5 & WELLS & (DELAPLAIN 15) \\
\hline 140 & 60 & $22 \mathrm{~A}$ & 167 & 6 & WELLS & (DELAPLAIN 15) \\
\hline 138 & 59 & & & 7 & WELLS & WINE CUP RANCH 7.5 \\
\hline 111 & 44 & 27 & & 8 & WELLS & TWELVEMILE RANCH 7.5 \\
\hline 69 & 20 & & & 9 & WELLS & MONTELLO 7.5 \\
\hline W & W & & & 10 & WELLS & MONTELLO 7.5 \\
\hline 102 & 39 & & & 11 & WELLS & OXLEY PEAK 7.5 \\
\hline 142 & 61 & $30 \mathrm{~A}$ & & 12 & WELLS & OXLEY PEAK 7.5 \\
\hline 122 & 50 & 30 & 168 & 13 & WELLS & OXLEY PEAK 7.5 \\
\hline $\mathrm{H}$ & $\mathrm{H}$ & & & 14 & WELLS & OXLEY PEAK 7.5 \\
\hline W & w & & & 15 & WELLS & WELLS 7.5 \\
\hline 104 & 40 & 22 & & 1 & WELLS & ROWLAND 15 \\
\hline 106 & 41 & 21 & & 2 & WELLS & MOUNTAIN CITY 15 \\
\hline 129 & 54 & & & 3 & WELLS & WILD HORSE 15 \\
\hline 151 & 66 & $30 \mathrm{D}$ & & 4 & WELLS & HOT CREEK 15 \\
\hline 126 & 52 & 28 & & 5 & WELLS & HOT SPRINCS CREEK 7.5 \\
\hline 97 & 36 & & & 6 & WELLS & TWIN BUTTES 7.5 \\
\hline $\mathrm{H}$ & $\mathrm{H}$ & 29 & & 7 & WELLS & TWIN BUTTES 7.5 \\
\hline w & W & & & 8 & WELLS & MORGAN HILL 7.5 \\
\hline 194 & 90 & & 164 & 1 & MCDERMITT & TUSCARORA 15 \\
\hline W & w & & & 2 & MCDERMITT & (TUSCARORA 15) \\
\hline $\mathrm{H}$ & $\mathrm{H}$ & & & 3 & MCDERMITT & SQUAW VALLEY RANCH 7.5 \\
\hline 135 & 57 & 11 & & 1 & MCDERMITT & HOT SPRINCS PEAK 15 \\
\hline 158 & 70 & & & 2 & MCDERMITT & (BLISS 15) \\
\hline 79 & 26 & & & 3 & MCDERMITT & \\
\hline 70 & 21 & 19D & & 4 & MCDERMITT & OSGOOD MOUNTAINS 15 \\
\hline w & W & & & 1 & VYA & RAILROAD POINT 15 \\
\hline
\end{tabular}




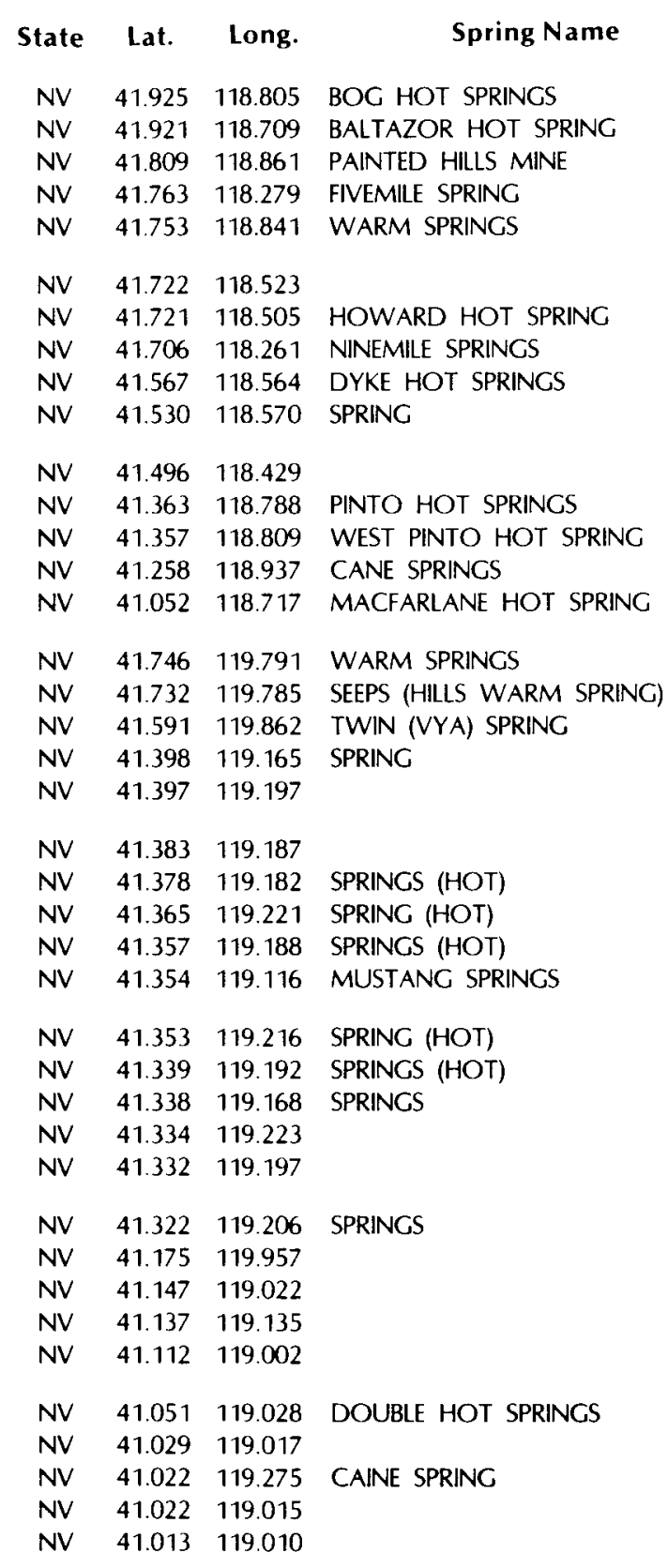

\begin{tabular}{|c|c|}
\hline Temp & $\begin{array}{l}\text { ature } \\
\text { oc }\end{array}$ \\
\hline 131 & 55 \\
\hline 181 & 83 \\
\hline 131 & 55 \\
\hline 81 & 27 \\
\hline 112 & 44 \\
\hline 163 & 73 \\
\hline 135 & 57 \\
\hline 79 & 26 \\
\hline 158 & 70 \\
\hline 104 & 40 \\
\hline 70 & 21 \\
\hline 199 & 93 \\
\hline 198 & 92 \\
\hline 74 & 23 \\
\hline 170 & 77 \\
\hline 73 & 23 \\
\hline 83 & 28 \\
\hline 71 & 22 \\
\hline 84 & 29 \\
\hline 114 & 46 \\
\hline 130 & 54 \\
\hline 136 & 58 \\
\hline 122 & 50 \\
\hline 133 & 56 \\
\hline 73 & 23 \\
\hline 127 & 53 \\
\hline 127 & 53 \\
\hline 79 & 26 \\
\hline 72 & 22 \\
\hline 102 & 39 \\
\hline 75 & 24 \\
\hline $\mathrm{H}$ & $\mathrm{H}$ \\
\hline 153 & 67 \\
\hline 80 & 27 \\
\hline 151 & 66 \\
\hline 178 & 81 \\
\hline 114 & 46 \\
\hline 73 & 23 \\
\hline 161 & 72 \\
\hline 172 & 78 \\
\hline
\end{tabular}

P.P. Circ. NOAA 1:250,000(AMS)map 492790

$\begin{array}{lll}2 & 130 \\ 6 & \\ & \\ 10 & 131\end{array}$

$10 \mathrm{~A}$

$$
\begin{array}{lll} 
& 12 & \text { VYA } \\
132 & 13 & \text { VYA } \\
132 & 14 & \text { VYA } \\
& 15 & \text { VYA } \\
& 16 & \text { VYA }
\end{array}
$$

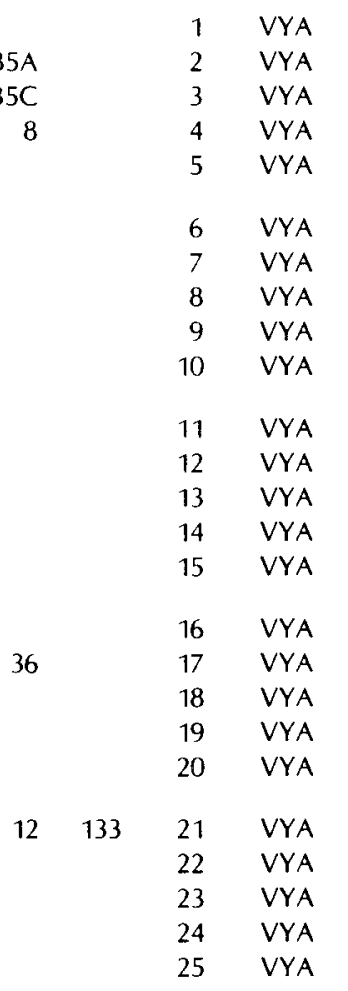

$1: 63360$ or $1: 62500$ (15-minute) or 1:24000 (7.5-minute) quadrangle

RAILROAD POINT 15

DENIO 15

(RAILROAD POINT 15)

TRIDENT PEAK 15

RAILROAD POINT 15

DUFFER PEAK 15

DUFFER PEAK 15

QUINN RIVER CROSSING 15

DUFFER PEAK 15

DUFFER PEAK 15

(BOTTLE CREEK 15)

PINTO MOUNTAIN 7.5

PINTO MOUNTAIN 7.5

PAIUTE MEADOWS 7.5

KING LEAR PEAK 15

ALKALI LAKE 7.5

ALKALI LAKE 7.5

VYA 7.5

SOLDIER MEADOW 7.5

(SOLDIER MEADOW 7.5)

(SOLDIER MEADOW 7.5)

SOLDIER MEADOW 7.5

MUD MEADOW 7.5

MUD MEADOW 7.5

BIG MOUNTAIN 7.5

MUD MEADOW 7.5

MUD MEADOW 7.5

MUD MEADOW 7.5

MUD MEADOW 7.5

MUD MEADOW 7.5

MUD MEADOW 7.5 
NV $\quad 41.003 \quad 119.008$ $\begin{array}{llll}\text { NM } & 32.455 & 104.489 & \text { INDIAN BIG SPRING }\end{array}$

NM $\quad 32.248 \quad 104.411$ MOSLEY SPRING

$\begin{array}{llll}\text { NM } & 32.501 & 106.926 & \text { RADIUM SPRINCS }\end{array}$

NM $\quad 32.953 \quad 107.580$

NM $\quad 32.795 \quad 107.276 \quad$ DERRY WARM SPRINCS

NM $\quad 32.748 \quad 107.836 \quad$ MIMBRES HOT SPRINGS

NM $\quad 32.693 \quad 107.811$

NM $32.606 \quad 107.190 \quad$ SOUSE SPRINGS

NM $32.554 \quad 107.994 \quad$ FAYWOOD HOT SPRINCS

NM $\quad 32.975 \quad 108.631$

NM $32.884 \quad 108.358 \quad$ ALLEN SPRINGS

NM $32.843 \quad 108.512$ MANGAS SPRINGS

NM $32.821 \quad 108.408$ ASH SPRING
Temperature

204
79

68

$127 \quad 53$

94

$92 \quad 33$

136
72

88

$129 \quad 54$

$77 \quad 25$

$78 \quad 26$

$\begin{array}{ll}81 & 27 \\ 72 & 22\end{array}$
P.P. Circ. NOAA 1:250,000(AMS)map 492790

KENNECOTT WARM SPRINGS

NM $\quad 32.899 \quad 109.035$

$\begin{array}{llll}\text { NM } & 32.830 & 109.047 & \text { GOAT CAMP SPRING }\end{array}$

NM $\quad 33.813 \quad 106.971$

NM $\quad 33.572 \quad 107.593 \quad$ OJO CALIENTE

NM $\quad 33.279 \quad 107.563 \quad$ WARM SPRINCS

NM $\quad 33.135 \quad 107.254$ TRUTH OR CONSEQUENCES

NM $33898 \quad 108.501 \quad$ ARAGON SPRINGS

$\begin{array}{llll}N M & 33.831 & 108.800 & \text { (UPPER) FRISCO HOT SPRINC }\end{array}$

NM $33.306 \quad 108.324$ THE MEADOWS WARM SPRING

$\begin{array}{llll}\text { NM } & 33.293 & 108.282 & \text { SPRING MFG TWO, SPRING MFG THREE }\end{array}$

NM $33.285 \quad 108.262 \quad$ SPRING MFG ONE

NM $\quad 33.274 \quad 108.250 \quad$ SPRING MFG FOUR

$\begin{array}{lll}\text { NM } & 33.258 & 108.233\end{array}$

NM $\quad 33.244 \quad 108.880$ SAN FRANCISCO HOT SPRINGS

$\begin{array}{llll}\text { NM } & 33.237 & 108.880 & \text { HOT SPRINCS }\end{array}$

NM $33.233 \quad 108.235 \quad$ SPRING (HOT)

NM $33.212 \quad 108.228$

NM $33.199 \quad 108.209$ GILA HOT SPRINCS

NM $33.192 \quad 108.180$ LYONS HUNTING LODGE HOT SPRINCS

NM $\quad 33.162 \quad 108.209 \quad$ SPRING (HOT)

NM $33.108 \quad 108.483$ TURKEY CREEK HOT SPRING

NM $33.708 \quad 109.025$ FRIEBORN CANYON SPRINC

NM $34.995 \quad 106.454 \quad$ CLEAR WATER SPRINC

NM $\quad 34.264 \quad 106.883$
38 175

34

35

6 VYA

CARLSBAD

CARLSBAD

CARLSBAD
LAS CRUCES

LAS CRUCES

LAS CRUCES

LAS CRUCES

LAS CRUCES

LAS CRUCES

LAS CRUCES

SILVER CITY

SILVER CITY

SILVER CITY

SILVER CITY

SILVER CITY

SILVER CITY

SILVER CITY

SILVER CITY

TULAROSA

TULAROSA

24

37

TULAROSA

TULAROSA

CLIFTON

CLFTON

CLIFTON

CLIFTON

CLIFTON

CLIFTON

CLIFTON

$25 \quad 174$

CLIFTON

27

CLIFTON

CLIFTON

CLIFTON

$\begin{array}{lll}30 & 12 & \text { CLIFTON } \\ 31 & 13 & \text { CIFTON }\end{array}$

CIFTON

CLIFTON

CLIFTON

CLIFTON
SOCORRO

SOCORRO
1:63360 or $1: 62500$ (15-minute)

or 1:24000 (7.5-minute) quadrangle

WEST CARLSBAD 15

CARLSBAD CAVERNS EAST 15

SAN DIEGO MOUNTAIN 15

(HILLSBORO 15)

GARFIELD 15

DWYER 15

(DWYER 15)

SOUSE SPRINGS 7.5

DWYER 15

(CLIFF 15)

READING MOUNTAIN 7.5

CLIFF 15

CIRCLE MESA 7.5

(HURLEY WEST 7.5)

FAYWOOD STATION 7.5

(YORK VALLEY 15)

YORK VALLEY 15

(SAN ANTONIO 15)

MONTOYA BUTTE 7.5

CHISE 7.5

CUCHILLO 7.5

(ARAGON 7.5)

DILLON MOUNTAIN 7.5

(WOODLAND PARK 7.5 )

(WOODLAND PARK 7.5) (WOODLAND PARK 7.5 )

(WOODLAND PARK 7.5)

(BURNT CORRAL CANYON 7.5)

WILSON MOUNTAIN 7.5

WILSON MOUNTAIN 7.5 GILA HOT SPRINGS 7.5

(GILA HOT SPRINGS 7.5)

GILA HOT SPRINGS 75

(GILA HOT SPRINGS 7.5)

GILA HOT SPRINCS 7.5

(CANYON HILL 7.5)

(BLUE 15)

(MOUNT WASHINGTON 7.5)

(SAN ACACIA 7.5) 


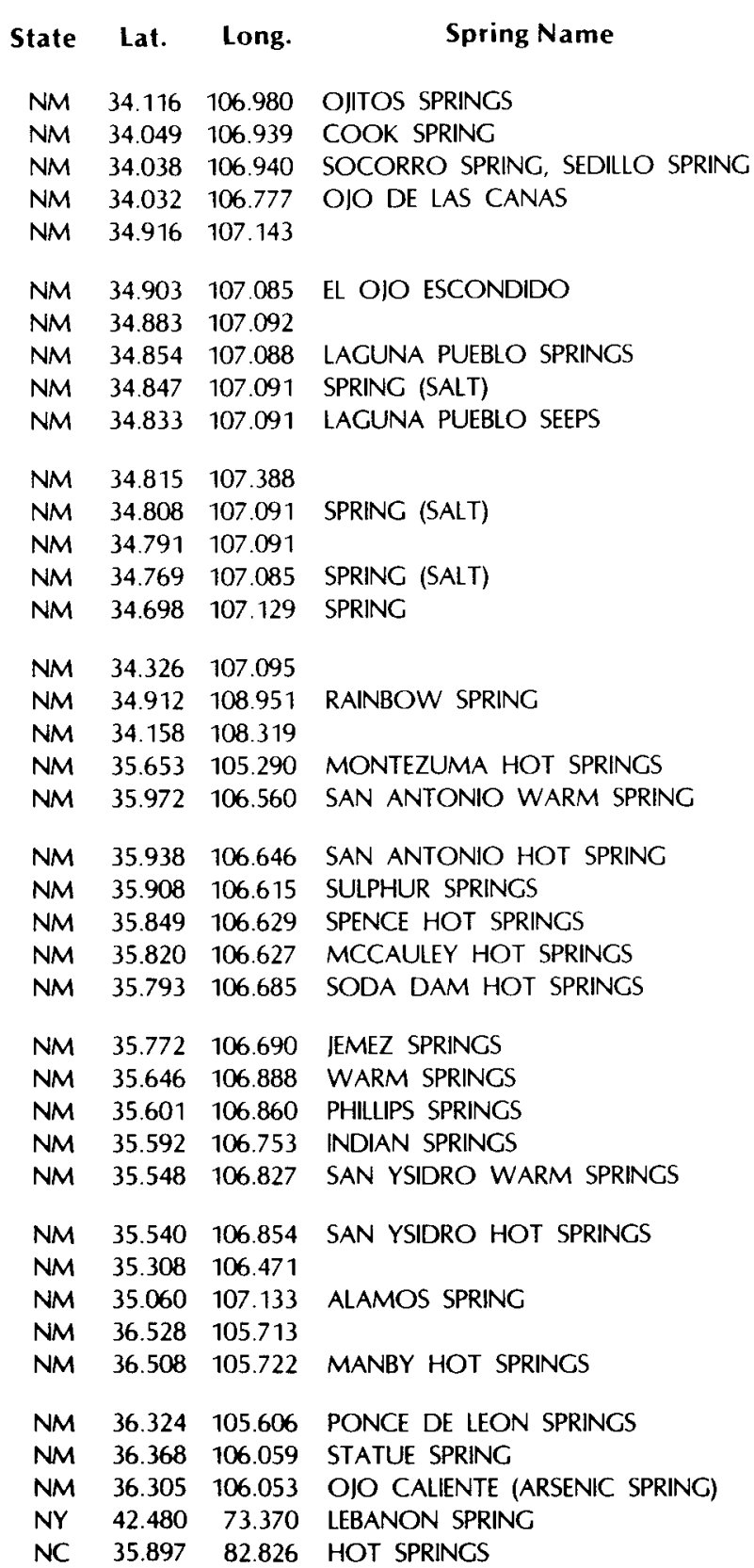

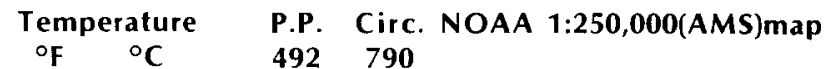

$68 \quad 20$

$93 \quad 34$

$79 \quad 26$

$68 \quad 20$

$73 \quad 23$

73
80

$76 \quad 24$

$82 \quad 28$

$68 \quad 20$

$86 \quad 30$

$78-27$

$71 \quad 22$

$72 \quad 22$

71
72

$\begin{array}{ll}138 & 59 \\ 101 & 38\end{array}$

101

20
21
34
26
20
23
23
27
24
28
20
30
27
26
22
22
22
22
59
38
54
87
38
37
47
76
54
21
51
20
29
20
22
41
38
34
28
44
22
42

\section{SOCORRO}

23

2

20

11
SOCORRO

SOCORRO

SOCORRO

SOCORRO

SOCORRO

SOCORRO

SOCORRO

SOCORRO

SOCORRO

SOCORRO

SOCORRO

SOCORRO

SOCORRO

SOCORRO

SOCORRO

SAINT JOHNS

SAINT JOHNS

SANTA FE

ALBUQUERQUE

ALBUQUERQUE

ALBUQUERQUE

ALBUQUERQUE

ALBUQUERQUE

ALBUOUEROUE

ALBUQUERQUE

ALBUQUERQUE

ALBUQUERQUE

ALBUQUERQUE

ALBUQUERQUE

ALBUQUERQUE

ALBUQUERQUE

ALBUQUERQUE

RATON

RATON

RATON

AZTEC

AZTEC

ALBANY

KNOXVILLE
$1: 63360$ or $1: 62500$ (15-minute)

or 1:24000 (7.5-minute) quadrangle

SOCORRO 7.5

SOCORRO 7.5

SOCORRO 7.5

LOMA DE LAS CANAS 7.5

(SOUTH GARCIA 15)

SOUTH GARCIA 7.5

(SOUTH GARCIA 7.5)

SOUTH GARCIA SE 7.5

SOUTH GARCIA SE 7.5

SOUTH GARCIA SE 7.5

(MARMON RANCH 7.5)

SOUTH GARCIA SE 7.5

(SOUTH GARCIA SE 7.5)

SOUTH GARCIA SE 7.5

MESA APAREJO 15

(RILEY 15)

OIO CALIENTE RESERVOIR 7.5

MONTEZUMA 7.5

VALLE SAN ANTONIO 7.5

SEVEN SPRINGS 7.5

VALLE SAN ANTONIO 7.5

IEMEZ SPRINGS 7.5

IEMEZ SPRINCS 7.5

IEMEZ SPRINCS 7.5

JEMEZ SPRINCS 7.5

HOLY GHOST SPRING 7.5

SAN YSIDRO 7.5

(SAN YSIDRO 7.5)

SAN YSIDRO 7.5

(SAN YSIDRO 7.5)

(PLACITAS 7.5)

MESA GICANTE 7.5

(ARROYO HONDO 7.5)

ARROYO HONDO 75

RANCHOS DE TAOS 7.5

OJO CALIENTE 7.5

OJO CALIENTE 7.5

PITTSFIELD WEST 7.5

HOT SPRINGS 7.5 
State Lat. Long.

Spring Name

$\begin{array}{lllll}\text { OR } & 42.977 & 117.061 & \text { CANTERS HOT SPRING }\end{array}$

$\begin{array}{llll}O R & 42.756 & 117.550 & \text { SCOIT SPRINC }\end{array}$

$\begin{array}{llll}\text { OR } & 42.688 & 117.905 & \text { TUDORS SPRINC }\end{array}$

$\begin{array}{lll}\text { OR } & 42.533 & 117.182\end{array}$

$\begin{array}{llll}\text { OR } & 42.889 & 118.898 & \text { FIVEMILE SPRING (HOGHOUSE HOT SPRS) }\end{array}$

$42.837 \quad 118.863$

42.815118 .899 WARM SPRINGS

$42.676 \quad 118.344$ MICKEY SPRINGS

$\begin{array}{lll}42.544 & 118.533 & \text { ALVORD HOT SPRINGS }\end{array}$

$\begin{array}{lll}42.338 & 118.602 & \text { HOT SPRINCS }\end{array}$

$\begin{array}{lll}42.327 & 118.602 & \text { BORAX LAKE (HOT LAKE) }\end{array}$

$\begin{array}{lll}42.254 & 118.703\end{array}$

$\begin{array}{lll}\text { OR } & 42.253 & 118.312\end{array}$
OR $\quad 42.079 \quad 117.760$

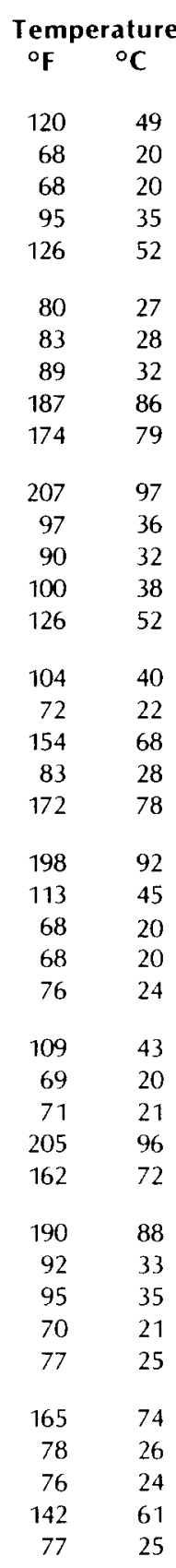

P.P. Circ. NOAA 1:250,000(AMS)map 492790

\begin{tabular}{|c|c|c|c|}
\hline 85 & & 1 & JORDAN VALLEY \\
\hline $85 \mathrm{~A}$ & & & JORDAN VALLEY \\
\hline $85 \mathrm{~B}$ & & & JORDAN VALLEY \\
\hline $85 \mathrm{C}$ & & 4 & JORDAN VALLEY \\
\hline 8 & 200 & 5 & JORDAN VALLEY \\
\hline
\end{tabular}

42.501 119.693 ANTELOPE HOT SPRINCS

$42.307 \quad 119.875$ MOSS RANCH SPRINC

$\begin{array}{lll}42.297 & 119.776 & \text { FISHER HOT SPRINGS }\end{array}$

$\begin{array}{llll} & 42.287 & 119.867 & \text { MOSS RANCH SPRINCS } \\ \text { R } & 42.226 & 119.881 & \text { CRUMP GEYSER (CRUMP SPRING) }\end{array}$

$\begin{array}{llll}42.219 & 119.877 & \text { WARNER VALLEY RANCH }\end{array}$

42.075119 .927 HALLINAN SPRING

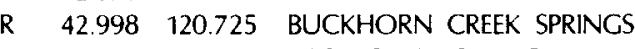

$\begin{array}{lll}42.933 & 120.642 & \text { LOST CABIN SPRINC }\end{array}$

$\begin{array}{lll}42.930 & 120.798 & \text { PARDON WARM SPRING }\end{array}$

OR $\quad 42.725 \quad 120.646 \quad$ SUMMER LAKE HOT SPRING

$\begin{array}{llll}\text { OR } & 42.380 & 120.330 & \text { BEAN HOT SPRINC }\end{array}$

$\begin{array}{llll}\text { OR } & 42.325 & 120.327 & \text { WHITE ROCK RANCH HOT SPRINC }\end{array}$

$\begin{array}{llll}\text { OR } & 42.221 & 120.368 & \text { HUNTERS HOT SPRINCS }\end{array}$

$\begin{array}{llll}O R & 42.162 & 120.344 & \text { LEITHEAD HOT SPRING }\end{array}$

$\begin{array}{llll}\text { OR } & 42.156 & 120.345 & \text { BARRY RANCH HOT SPRINCS }\end{array}$

OR $42.040 \quad 120.924$ WARM SPRING

OR 42.431 121.963 EACLE POINT SPRING

OR $42.178 \quad 121.801$ HARDBOARD SPRINC

OR $\quad 42.176 \quad 121828$ HOWARD HOUDAY

OR $\quad 42.174 \quad 121.615$ OLENE GAP HOT SPRINCS

$\begin{array}{lllll}\text { OR } & 42.161 & 121.627 & \text { CRYSTAL SPRING }\end{array}$

$\begin{array}{llll}\text { OR } & 42.133 & 121.219 & \text { WILKERSONS HOT SPRINC }\end{array}$

$\begin{array}{llll}\text { OR } & 42.116 & 121.287 & \text { OREGON HOT SPRINCS (BIG HOT SPRING) }\end{array}$

OR $42.873 \quad 122.167 \quad$ ANNIE SPRING
$65 \quad 1$ ADEL

$66 \quad 2$ ADEL

$67 \quad 3 \quad$ ADEL

$\begin{array}{lll}196 & 4 & \text { ADEL } \\ 197 & 5 & \text { ADEL }\end{array}$

$69 \quad 198 \quad 6 \quad$ ADEL

$\begin{array}{rrrr}72 & 199 & 9 & \text { ADEL } \\ & 10 & \text { ADEL }\end{array}$

$48 \mathrm{~A}$

1 ADEL

49A $191 \quad 3 \quad$ ADEL

49C $190 \quad 5$ ADEL

$49 \mathrm{D}$

$50 \mathrm{~A}$
38

$40 E$

41

$\begin{array}{ll}42 & 188\end{array}$

$44 \mathrm{E}$

$44 \mathrm{D}$

45

46

$47 \quad 189$

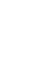

187

187

$28 \quad 187$

28
$28 \mathrm{~B}$
30

30 $\begin{array}{llll}70 & 198 & 7 & \text { ADEL }\end{array}$

8 ADEL

$49 \mathrm{~B} \quad 4$ ADEL
6 ADEL

ADEL
KLAMATH FALLS

KLAMATH FALLS

KLAMATH FALLS

KLAMATH FALLS

KLAMATH FALLS

KLAMATH FALLS

KLAMATH FALLS

KLAMATH FALLS

KLAMATH FALLS

KLAMATH FALLS

KLAMATH FALLS

KLAMATH FALLS

KLAMATH FALLS

KLAMATH FALLS

KLAMATH FALLS

KLAMATH FALLS

MEDFORD
KLAMATH FALLS
1:63360 or 1:62500 (15-minute)

or 1:24000 (7.5-minute) quadrangle

\section{(JORDAN VALLEY 7.5)}

(SCOTT RESERVOIR 7.5)

IRISH LAKE 7.5

(PACE SPRINCS 7.5)

FRENCHCLEN 7.5

ALVORD HOT SPRINGS 7.5

BORAX LAKE 7.5

BORAX LAKE 7.5

(FIELDS 7.5)

CAMPBELL LAKE 7.5

PRIDAY RESERVOIR 7.5

CRUMP LAKE 7.5

CRUMP LAKE 7.5

ADEL 7.5

(ADEL 7.5)

MAY LAKE 7.5

(SUMMER LAKE 7.5)

SLIDE MTN 7.5

CROOKED CREEK VALLEY 7.5

LAKEVIEW NE 7.5

LAKEVIEW NE 7.5

LAKEVIEW NE 7.5

STRAWBERRY BUTTE 15

(MODOC POINT 15)

(KLAMATH FALLS 15)

(KLAMATH FALLS 15)

\section{(MERRILL 15)}

(MERRILL 15)

GERBER RESERVOIR 15

MALIN 15

CRATER LAKE NATIONAL PARK 


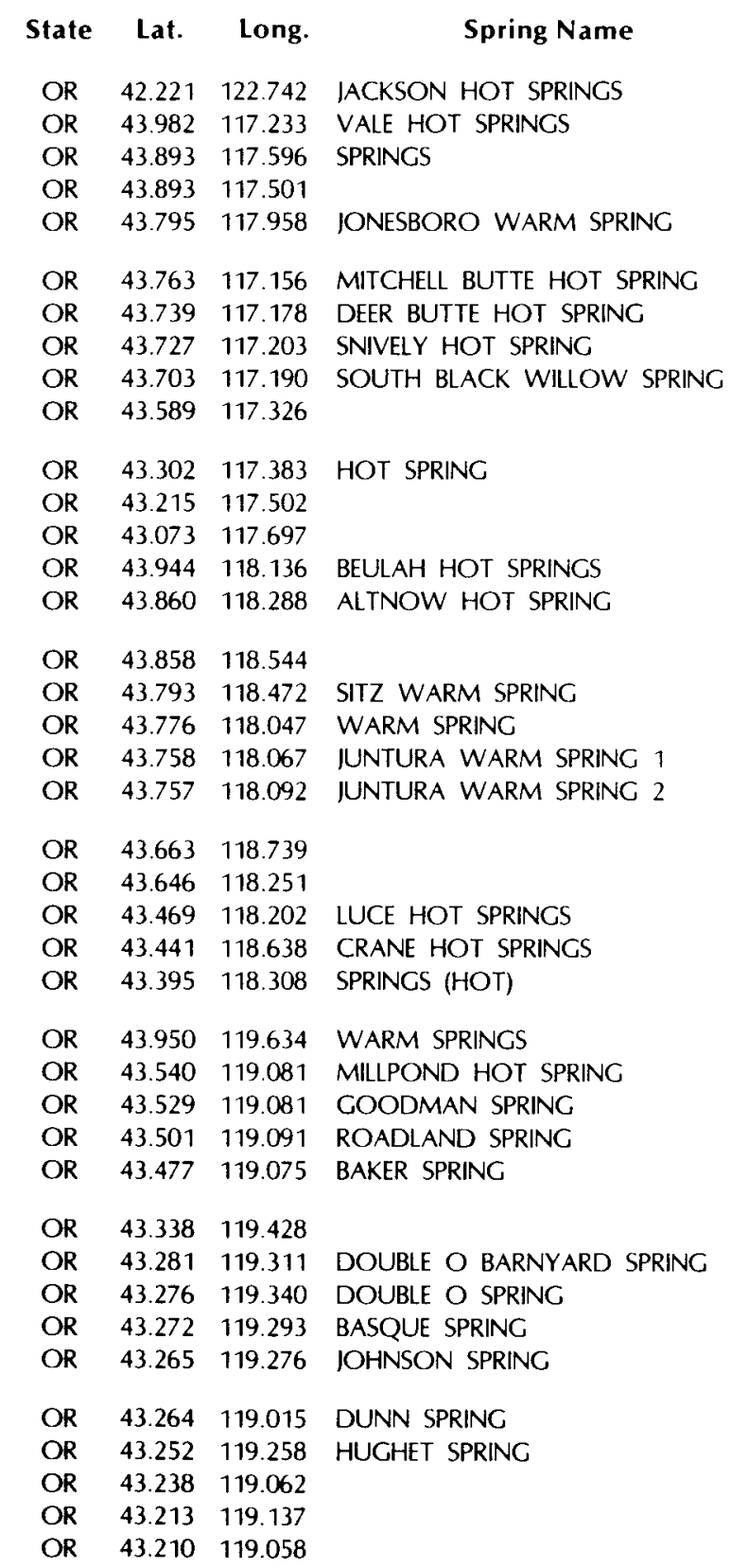

\begin{tabular}{|c|c|c|c|c|c|c|}
\hline$\underset{{ }^{\circ} \mathrm{F}}{\operatorname{Temp}}$ & $\begin{array}{l}\text { ature } \\
{ }^{\circ} \mathrm{C}\end{array}$ & $\begin{array}{l}\text { P.P. } \\
492\end{array}$ & $\begin{array}{l}\text { Circ. } \\
790\end{array}$ & NOAA & $1: 250,000(A M S)$ map & $\begin{array}{l}1: 63360 \text { or } 1: 62500(15 \text {-minute }) \\
\text { or } 1: 24000(7.5 \text {-minute }) \text { quadrangle }\end{array}$ \\
\hline 95 & 35 & 25 & & 2 & MEDFORD & ASHLAND 15 \\
\hline 163 & 73 & 77 & 204 & 1 & BOISE & (VALE EAST 7.5 ) \\
\hline W & W & & & 2 & BOISE & HARPER 15 \\
\hline 158 & 70 & 76 & 202 & 3 & BOISE & (HARPER 15) \\
\hline 112 & 45 & & & 4 & BOISE & (WESTFALL BUTTE 15) \\
\hline 144 & 62 & 79 & & 5 & BOISE & MITCHELL BUTTE 7.5 \\
\hline 115 & 46 & 80 & & 6 & BOISE & OWYHEE DAM 7.5 \\
\hline 170 & 77 & & & 7 & BOISE & OWYHEE DAM 7.5 \\
\hline 71 & 22 & 82 & & 8 & BOISE & (OWYHEE DAM 7.5) \\
\hline $\mathrm{H}$ & $\mathrm{H}$ & 83 & & 9 & BOISE & (THE ELBOW 7.5) \\
\hline 106 & 41 & & & 10 & BOISE & DIAMOND BUTTE 7.5 \\
\hline $\mathrm{H}$ & $\mathrm{H}$ & $84 \mathrm{~A}$ & & 11 & BOISE & (THE HOLE IN THE GROUND 7.5) \\
\hline$H$ & $\mathrm{H}$ & & & 12 & BOISE & (LAMBERT ROCKS 7.5) \\
\hline 140 & 60 & 74 & & 1 & BURNS & BEULAH 15 \\
\hline$w$ & W & & & 2 & BURNS & DREWSEY 15 \\
\hline 165 & 74 & & & 3 & BURNS & (VAN 15) \\
\hline W & W & & & 4 & BURNS & DREWSEY 15 \\
\hline w & W & & & 5 & BURNS & BEULAH 15 \\
\hline 77 & 25 & & & 6 & BURNS & (BEULAH 15) \\
\hline 95 & 35 & & & 7 & BURNS & (BEULAH 15) \\
\hline 72 & 22 & $51 \mathrm{~A}$ & & 8 & BURNS & (BUCHANAN 7.5) \\
\hline 144 & 62 & 54 & & 9 & BURNS & (UPTON MOUNTAIN 7.5) \\
\hline 145 & 63 & 84 & 195 & 10 & BURNS & MCEWEN BUTTE 7.5 \\
\hline 172 & 78 & 53 & & 11 & BURNS & CRANE 15 \\
\hline 106 & 41 & 55 & & 12 & BURNS & DUNNEAN 7.5 \\
\hline 84 & 29 & & & 1 & BURNS & DELINTMENT LAKE 15 \\
\hline 79 & 26 & 52 & & 2 & BURNS & BURNS 7.5 \\
\hline 72 & 22 & $52 \mathrm{~B}$ & & 3 & BURNS & BURNS 7.5 \\
\hline 72 & 22 & $52 \mathrm{D}$ & & 4 & BURNS & BURNS 7.5 \\
\hline 70 & 21 & $52 \mathrm{E}$ & & 5 & BURNS & (DOG MOUNTAIN 15) \\
\hline 68 & 20 & 56 & & 6 & BURNS & \\
\hline 72 & 22 & 59 & & 7 & BURNS & \\
\hline 74 & 23 & 58 & & 8 & BURNS & \\
\hline 74 & 23 & 60 & & 9 & BURNS & \\
\hline 72 & 22 & 61 & & 10 & BURNS & \\
\hline 70 & 21 & $63 \mathrm{~A}$ & 193 & 11 & BURNS & \\
\hline 68 & 20 & 62 & 193 & 12 & BURNS & \\
\hline 70 & 21 & & 193 & 13 & BURNS & \\
\hline 104 & 40 & $62 \mathrm{D}$ & 193 & 14 & BURNS & \\
\hline 92 & 33 & & 193 & 15 & BURNS & \\
\hline
\end{tabular}


Spring Name

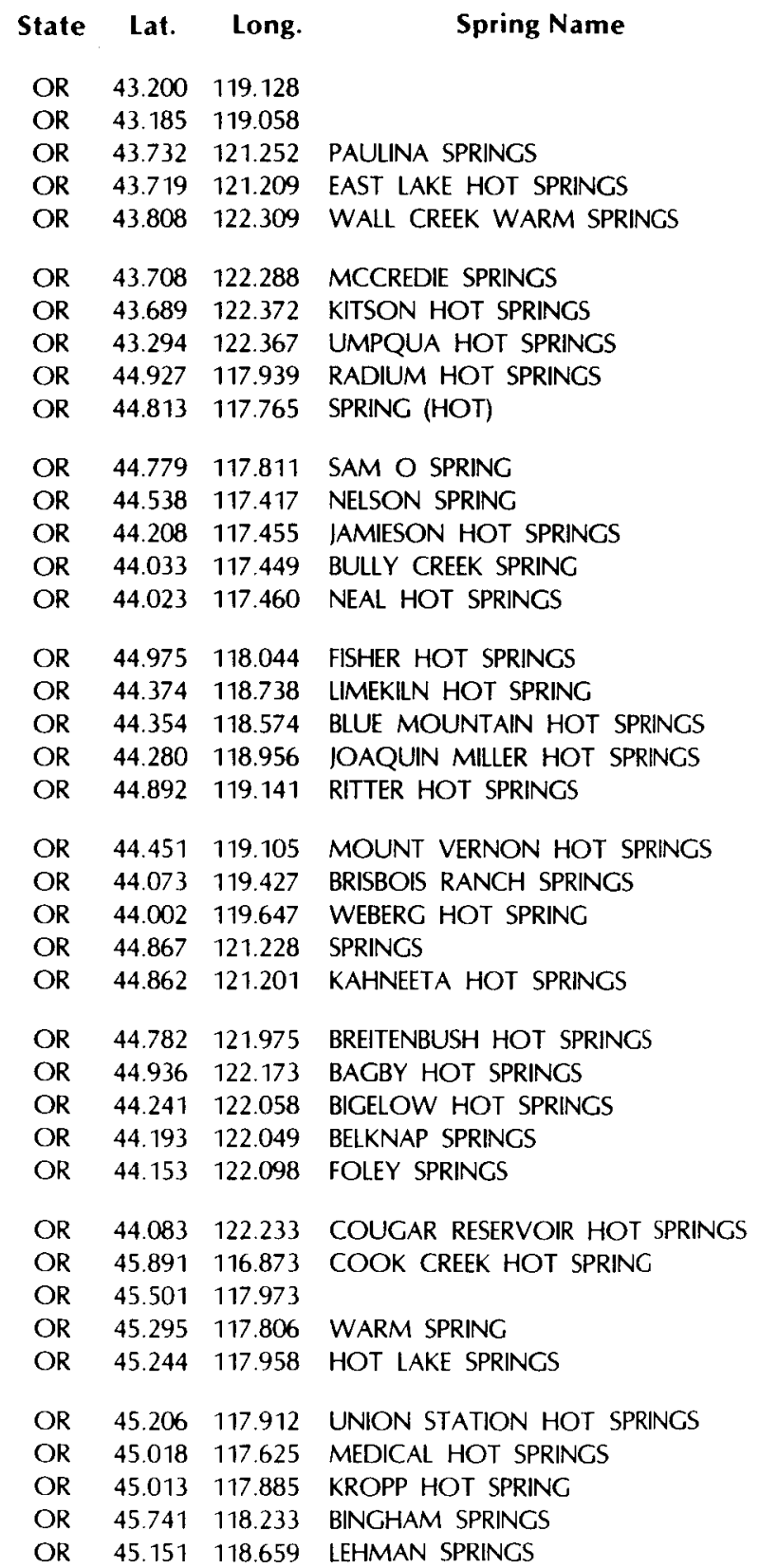

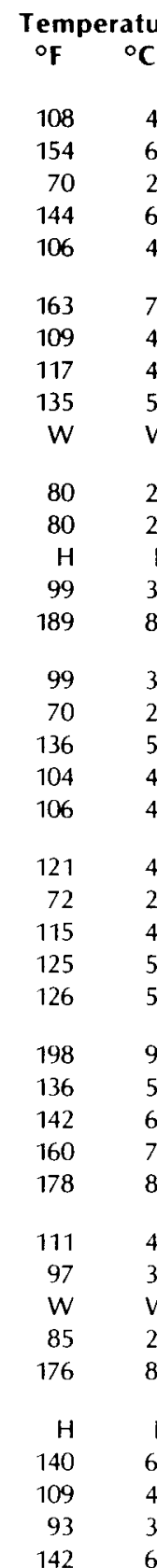

\section{P.P. Circ. NOAA 1:250,000(AMS)map} 492790

62D 193

$\begin{array}{ll}64 & 193\end{array}$

$32 \quad 184$

$\begin{array}{ll}33 & 184\end{array}$

21

23

24

$17 \mathrm{~A}$

$17 \mathrm{~B}$

17

73

$75 \quad 203$

16

15

13

792
$7 \quad 180$

$6 \quad 179$

5

$18 \quad 181$

$19 \quad 182$

20

11

$\begin{array}{ccl}193 & 16 & \text { BURNS } \\ 193 & 17 & \text { BURNS } \\ 184 & 1 & \text { CRESCENT } \\ 184 & 2 & \text { CRESCENT } \\ & 1 & \text { ROSEBURG } \\ & & \\ 183 & 2 & \text { ROSEBURG } \\ & 3 & \text { ROSEBURG } \\ 185 & 4 & \text { ROSEBURG } \\ & 1 & \text { BAKER } \\ & 2 & \text { BAKER }\end{array}$

BAKER

BAKER

BAKER

BAKER

BAKER

CANYON CITY

CANYON CITY

CANYON CITY

CANYON CITY

CANYON CITY

CANYON CITY

CANYON CITY

CANYON CITY

BEND

BEND

BEND

SALEM

SALEM

SALEM

SALEM

\section{SAIEM}

GRANGEVILLE

GRANGEVILLE

GRANCEVILLE

GRANGEVILLE

GRANCEVILLE

GRANCEVILLE

GRANGEVILLE

PENDLETON
1:63360 or 1:62500 (15-minute)

or 1:24000 (7.5-minute) quadrangle

(PAULINA PEAK 7.5)

SARDINE BUTTE 15

OAKRIDGE 15

OAKRIDGE 15

TOKETEE FALLS 15

HAINES 7.5

BAKER 7.5

BAKER 7.5

(DURKEE 15)

(JAMIESON 15)

JAMIESON 15

JAMIESON 15

ROCK CREEK 7.5

PRAIRIE CITY 15

PRAIRIE CITY 15

CANYON MTN. 7.5

RITTER 15

MOUNT VERNON 7.5

(IZEE 15)

EACLE BUTTE 7.5

EACLE BUTTE 7.5

BREITENBUSH HOT SPRINGS 15

BATTLE AX 15

(MCKENZIE BRIDCE 15)

MCKENZIE BRIDGE 15

MCKENZIE BRIDGE 15

(MCKENZIE BRIDGE 15)

WAPSHILLA CREEK 7.5

(ELGIN 7.5)

COVE 7.5

CRAIG MOUNTAIN 7.5

CRAIG MOUNTAIN 7.5

FLAGSTAFF BUTTE 7.5

(NORTH POWDER 7.5)

BINGHAM SPRINCS 75

LEHMAN SPRINGS 7.5 


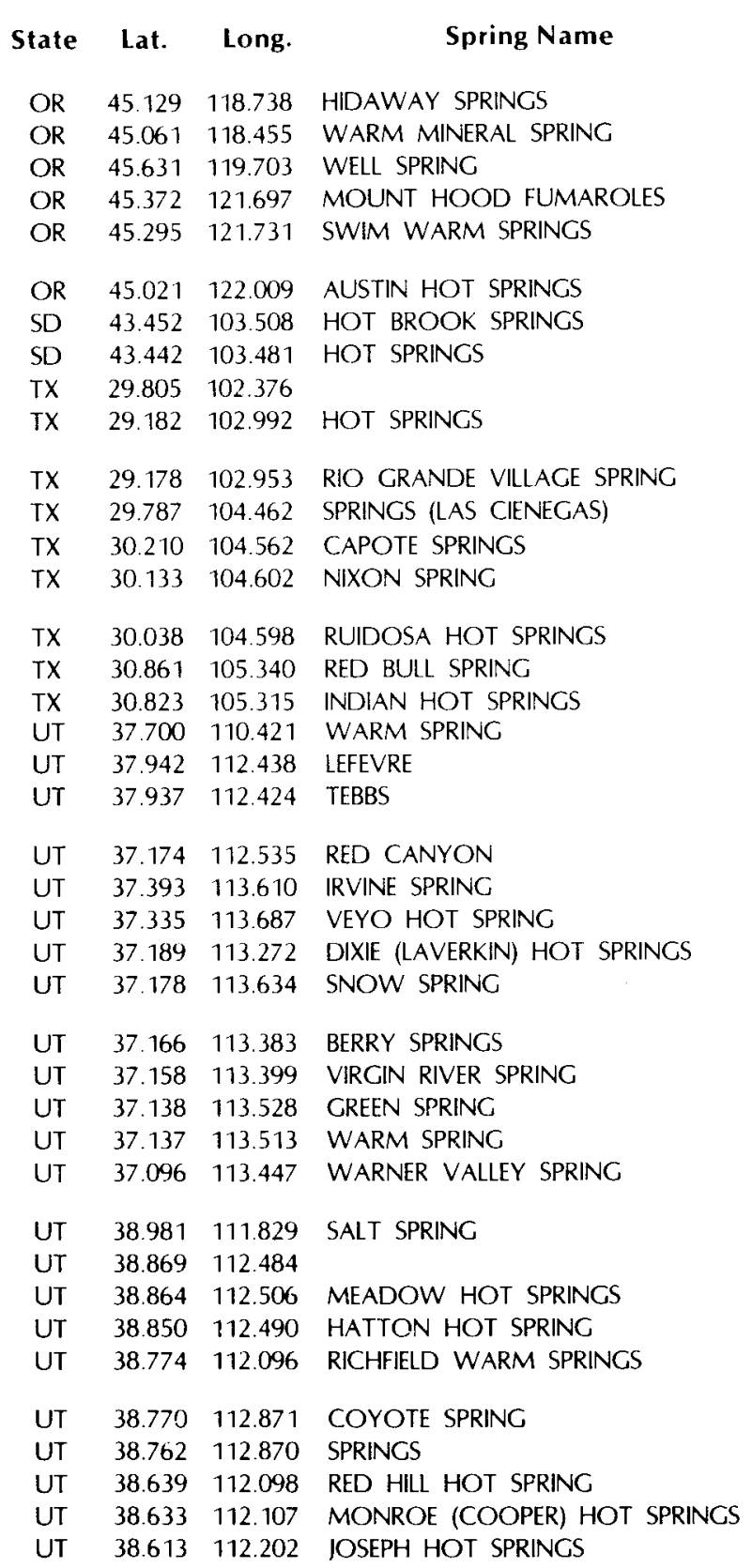

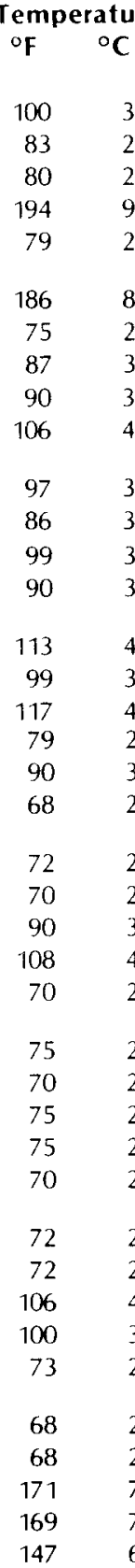

P.P. Circ. NOAA 1:250,000(AMS)map 492790

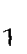

$\begin{array}{rll} & 3 & \text { PENDLETON } \\ & 4 & \text { PENDLETON } \\ & 1 & \text { PENDLETON } \\ 177 & 1 & \text { THE DALLES } \\ & 2 & \text { THE DALLES }\end{array}$

$4 \quad 178 \quad 1 \quad$ VANCOUVER HOT SPRINGS HOT SPRINCS EMORY PEAK EMORY PEAK

EMORY PEAK

PRESIDIO

MARFA

MARFA

MARFA

MARFA

MARFA

ESCALANTE

CEDAR CITY

CEDAR CITY

CEDAR CITY

CEDAR CITY

CEDAR CITY

CEDAR CITY

CEDAR CITY

CEDAR CITY

CEDAR CITY

CEDAR CITY

CEDAR CITY

CEDAR CITY

38

28

SALINA

RICHFIELD

RICHFIELD

RICHFIELD

RICHFIELD

RICHFIELD

RICHFIELD

$\begin{array}{lll}206 & 7 & \text { RICHFIELD }\end{array}$

$\begin{array}{llll}48 & 206 & 8 & \text { RICHFIELD }\end{array}$
1:63360 or $1: 62500$ (15-minute)

or 1:24000 (7.5-minute) quadrangle

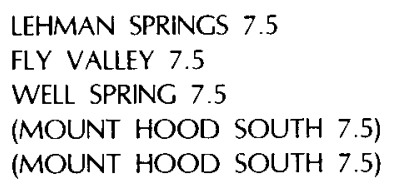

LEHMAN SPRINCS 7.5

FLY VALLEY 7.5

WELL SPRING 7.5

(MOUNT HOOD SOUTH 7.5)

(MOUNT HOOD SOUTH 7.5)

FISH CREEK MTN. 15

MINNEKHATA NE 7.5

HOT SPRINGS 7.5

(INDIAN WFLIS 15)

BOQUILLAS 7.5

BOQUILLAS 7.5

CERRO ORONA 7.5

(CAPOTE FALLS 7.5

CAPOTE FALLS 7.5

RUIDOSA HOT SPRINCS 7.5

NDIAN HOT SPRINCS 75

INDIAN HOT SPRINGS 7.5

(MANCOS MESA 15)

(PANGUITCH NW 7.5)

PANGUITCH NW 7.5

(KANAB 15)

CENTRAL EAST 7.5

VEYO 7.5

HURRICANE 15

ST. GEORGE 15

HURRICANE 15

HURRICANE 15

ST GEORCE 15

(ST. GEORGE 15)

HURRICANE 15

(SALINA 7.5)

(FILLMORE 15)

TABERNACLE HILL 15

FILLMORE 15

(RICHFIELD 15)

CRUZ 7.5

CRUZ 7.5

MONROE 15

MONROE 15

MONROE 15 


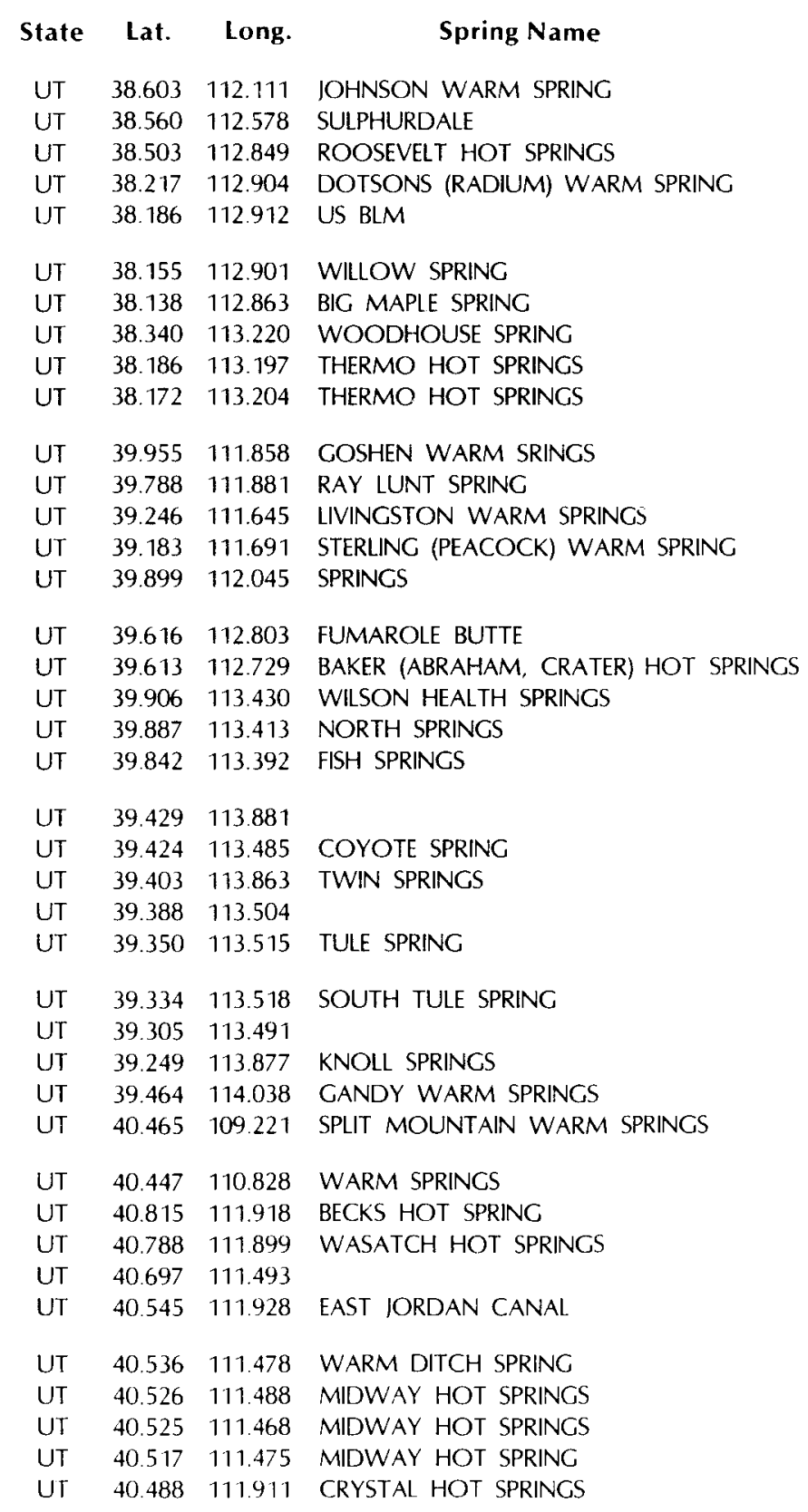

\begin{tabular}{|c|c|c|c|c|c|c|}
\hline Temp & ature & P.P. & Circ. & NOAA & $1: 250,000(A M S)$ map & $1: 63360$ or $1: 62500$ (15-minute) \\
\hline${ }^{\circ} \mathbf{F}$ & ${ }^{\circ} \mathrm{C}$ & 492 & 790 & & & or $1: 24000(7.5$-minute) quadrangle \\
\hline 77 & 25 & 47 & & 10 & RICHFIELD & MONROE 15 \\
\hline 72 & 22 & & 208 & 11 & RICHFIEID & COVE FORT 15 \\
\hline 133 & 56 & 51 & 209 & 12 & RICHFIEID & (PINNACLE PASS 7.5) \\
\hline 91 & 33 & 53 & & 13 & RICHFIELD & MINERSVILLE 7.5 \\
\hline 91 & 33 & & & 14 & RICHFIELD & (MINERSVILLE 7.5) \\
\hline 72 & 22 & & & 15 & RICHFIELD & MINERSVILLE 7.5 \\
\hline 70 & 21 & & & 16 & RICHFIELD & MINERSVILLE 15 \\
\hline 72 & 22 & & & 1 & RICHFIEID & MILFORD 15 \\
\hline 185 & 85 & 52 & 210 & 2 & RICHFIELD & THERMO 15 \\
\hline 181 & 83 & 52 & 210 & 3 & RICHFIELD & THERMO 15 \\
\hline 73 & 23 & 17 & & 1 & PRICE & SANTAQUIN 15 \\
\hline 68 & 20 & & & 2 & PRICE & SANTAQUIN 15 \\
\hline 73 & 23 & 31 & & 3 & PRICE & (STERLING 7.5) \\
\hline 72 & 22 & 35 & & 4 & PRICE & STERLING 7.5 \\
\hline 72 & 22 & & & 1 & DELTA & EUREKA 7.5 \\
\hline 73 & 23 & & & 2 & DELTA & FUMAROLE BUTTE 7.5 \\
\hline 180 & 82 & 24 & 205 & 3 & DELTA & BAKER HOT SPRINGS 7.5 \\
\hline 142 & 61 & 20 & & 1 & DELTA & FISH SPRINGS NW 7.5 \\
\hline 82 & 28 & 21 & & 2 & DELTA & FISH SPRINGS NW 7.5 \\
\hline 82 & 28 & 22 & & 3 & DELTA & FISH SPRINGS SW 7.5 \\
\hline 68 & 20 & 26 & & 4 & DELTA & (GANDY 15 ) \\
\hline 82 & 28 & & & 5 & DELTA & SWASEY PEAK NW 7.5 \\
\hline 68 & 20 & & & 6 & DELTA & GANDY 15 \\
\hline 77 & 25 & & & 7 & DELTA & (COWBOY PASS 15) \\
\hline 82 & 28 & & & 8 & DELTA & COWBOY PASS 15 \\
\hline 77 & 25 & & & 9 & DELTA & COWBOY PASS 15 \\
\hline 81 & 27 & & & 10 & DELTA & (SWASEY PEAK SW 7.5) \\
\hline 68 & 20 & 27 & & 11 & DELTA & CONGER RANGE 15 \\
\hline 81 & 27 & 25 & & 1 & ELY & \\
\hline 86 & 30 & $19 A$ & & 1 & VERNAL & SPLIT MOUNTAIN 7.5 \\
\hline 79 & 26 & & & 1 & SALT LAKE CITY & HANNA 7.5 \\
\hline 133 & 56 & 11 & & 1 & SALT LAKE CITY & SALT LAKE CITY NORTH 7.5 \\
\hline 108 & 42 & 12 & & 2 & SALT LAKE CITY & SALT LAKE CITY NORTH 7.5 \\
\hline 70 & 21 & & & 3 & SALT LAKE CITY & PARK CITY EAST 7.5 \\
\hline 73 & 23 & & & 4 & SALT LAKE CITY & (MIDVALE 7.5 ) \\
\hline 115 & 46 & & & 5 & SALT LAKE CITY & HEBER CITY 7.5 \\
\hline 100 & 38 & 14 & & 6 & SALT LAKE CITY & HEBER CITY 7.5 \\
\hline 104 & 40 & $14 \mathrm{~B}$ & & 7 & SALT LAKE CITY & HEBER CITY 7.5 \\
\hline 115 & 46 & $14 \mathrm{~A}$ & & 8 & SALT LAKE CITY & HEBER CITY 7.5 \\
\hline 187 & 86 & 13 & & 9 & SALT LAKE CITY & IORDAN NARROWS 7.5 \\
\hline
\end{tabular}




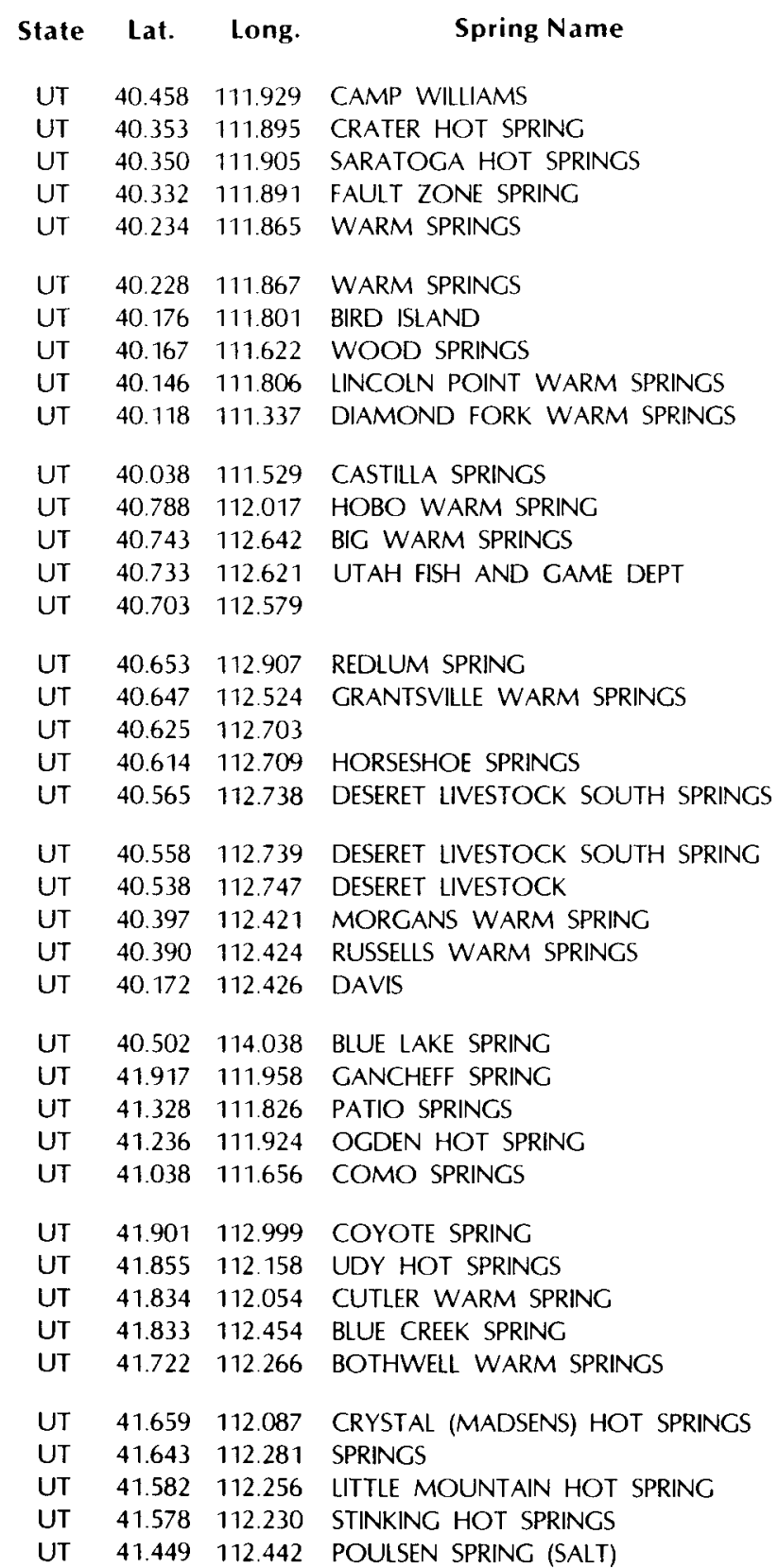

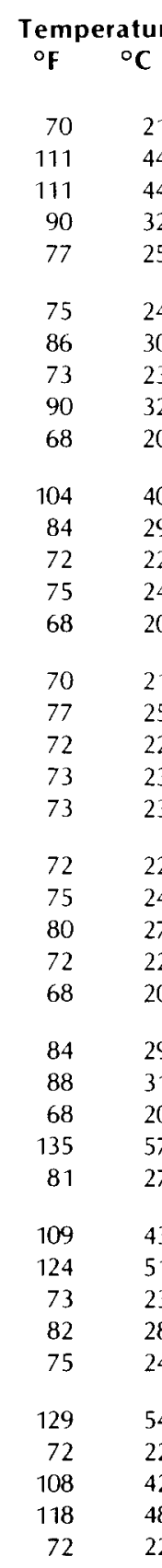

\section{P.P. Circ. NOAA 1:250,000(AMS)map 492790}

15

18

9

10

$10 \mathrm{~B}$

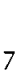

3

2

4

$4 \mathrm{~A}$
1:63360 or $1: 62500$ (15-minute) or $1: 24000$ (7.5-minute) quadrangle

(JORDAN NARROWS 7.5) (SARATOGA SPRINGS 7.5) SARATOGA SPRINGS 7.5 SARATOGA SPRINCS 7.5
(SARATOGA SPRINCS 7.5) (IINCOLN POINT 7.5)

(LINCOLN POINT 7.5) (LINCOLN POINT 7.5) (SPRINCVILLE 7.5)

(LINCOLN POINT 7.5)

RAYS VALLEY 7.5

SPANISH FORK PEAK 7.5

SALTAIR 7.5

TIMPIE 15

(TIMPIE 15)

(TIMPIE 15)

TIMPIE 15

(TIMPIE 15)

TIMPIE 15

TIMPIE 15

TIMPIE 15

(TIMPIE 15)

STOCKTON 15

STOCKTON 15

(FAUST 7.5)

WENDOVER SE 7.5

IRENTON 7.5

HUNTSVILLE 7.5

OGDEN 7.5

MORGAN 7.5

MONUMENT PEAK NW 7.5

RIVERSIDE 7.5

(CUTLER DAM 7.5)

HOWELL 7.5

(THATCHER MOUNTAIN 7.5)

HONEYVILLE 7.5

THATCHER MOUNTAIN 7.5

PUBLIC SHOOTING GROUNDS 7.5

BEAR RIVER CITY 7.5

EAST PROMONTORY 7.5 


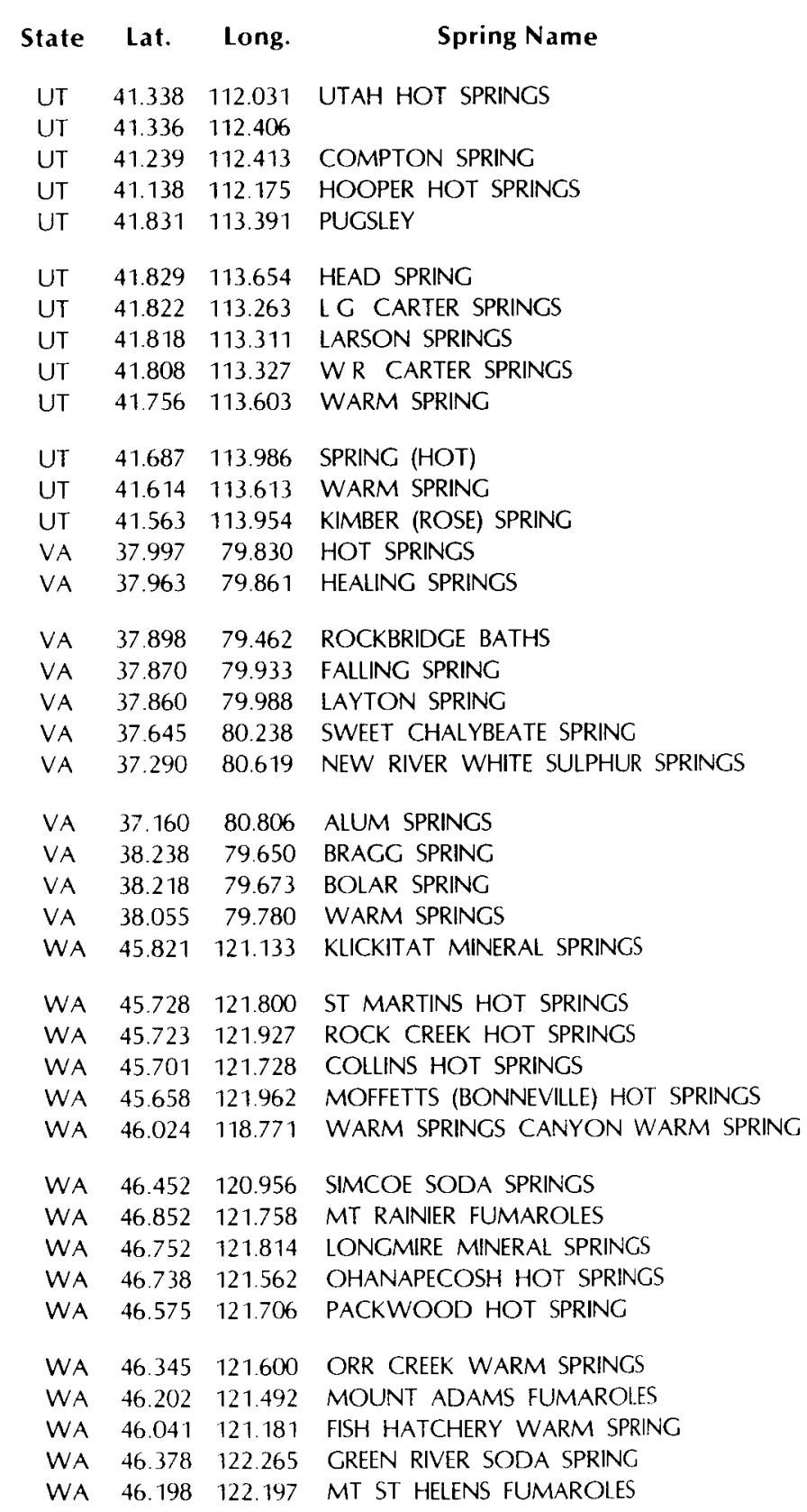

\begin{tabular}{|c|c|c|c|c|c|}
\hline \multicolumn{2}{|c|}{ Temperature } & \multirow{2}{*}{$\begin{array}{l}\text { P.P. } \\
492\end{array}$} & \multirow{2}{*}{$\begin{array}{c}\text { Circ. } \\
790\end{array}$} & \multirow{2}{*}{ NOAA } & \multirow{2}{*}{$1: 250,000($ AMS $)$ map } \\
\hline & & & & & \\
\hline 138 & 59 & 6 & & 11 & BRIGHAM CITY \\
\hline 77 & 25 & & & 12 & BRIGHAM CITY \\
\hline 72 & 22 & 5 & & 13 & BRIGHAM CITY \\
\hline 140 & 60 & & & 14 & BRIGHAM CITY \\
\hline 73 & 23 & & & 1 & BRIGHAM CITY \\
\hline 70 & 21 & & & 2 & BRIGHAM CITY \\
\hline 77 & 25 & & & 3 & BRIGHAM CITY \\
\hline 70 & 21 & & & 4 & BRIGHAM CITY \\
\hline 70 & 21 & & & 5 & BRIGHAM CITY \\
\hline 81 & 27 & 1 & & 6 & BRIGHAM CITY \\
\hline 108 & 42 & & & 7 & BRIGHAM CITY \\
\hline 68 & 20 & & & 8 & BRIGHAM CITY \\
\hline 68 & 20 & & & 9 & BRIGHAM CITY \\
\hline 106 & 41 & 8 & & 1 & ROANOKE \\
\hline 86 & 30 & 9 & & 2 & ROANOKE \\
\hline 72 & 22 & 11 & & 3 & ROANOKE \\
\hline 77 & 25 & 13 & & 4 & ROANOKE \\
\hline 72 & 22 & 12 & & 5 & ROANOKE \\
\hline 75 & 24 & 14 & & 1 & BLUEFIELD \\
\hline 85 & 29 & 18 & & 2 & BLUEFIELD \\
\hline 72 & 22 & 19 & & 3 & BLUEFIELD \\
\hline 75 & 24 & 5 & & 1 & CHARLOTTESVILLE \\
\hline 73 & 23 & 6 & & 2 & CHARLOTTESVILLE \\
\hline 95 & 35 & 7 & & 3 & CHARLOTTESVILLE \\
\hline 81 & 27 & & & 1 & THE DALLES \\
\hline 120 & 49 & & & 2 & THE DALLES \\
\hline $\mathrm{H}$ & $\mathrm{H}$ & & & 3 & THE DALLES \\
\hline 122 & 50 & & & 4 & THE DALLES \\
\hline 97 & 36 & 16 & & 5 & THE DALLES \\
\hline 72 & 22 & & & 1 & WALLA WALLA \\
\hline 90 & 32 & 12 & & 1 & YAKIMA \\
\hline 162 & 72 & & & 1 & YAKIMA \\
\hline 77 & 25 & & & 2 & YAKIMA \\
\hline 122 & 50 & 11 & 214 & 3 & YAKIMA \\
\hline 100 & 38 & & & 4 & YAKIMA \\
\hline 72 & 22 & & & 5 & YAKIMA \\
\hline 150 & 66 & $12 \mathrm{~B}$ & & 6 & YAKIMA \\
\hline 75 & 24 & & & 7 & YAKIMA \\
\hline 86 & 30 & & & 1 & HOQUIAM \\
\hline 190 & 88 & $12 \mathrm{~A}$ & & 2 & HOQUIAM \\
\hline
\end{tabular}

1:63360 or $1: 62500$ (15-minute) or $1: 24000$ (7.5-minute) quadrangle

PLAIN CITY 7.5

POKES POINT 7.5

PROMONTORY POINT 7.5

OGDEN BAY 7.5

PARK VALLEY 15

YOST 15

PARK VALLEY 15

PARK VALLEY 15

PARK VALLEY 15

YOST 15

GROUSE CREEK 7.5

PROHIBITION SPRINC 7.5

TOMS CABIN SPRING 7.5

HEALINC SPRINCS 7.5

HEALINC SPRINGS 7.5

GOSHEN 7.5

COVINGTON 7.5

(COVINGTON 7.5)

ALLEGHANY 7.5

(ECGLESTON 7 5)

WHITE GATE 7.5

(BURNSVILLE 7.5)

BURNSVILLE 7.5

WARM SPRINGS 7.5

(KLICKITAT 15)

BONNEVILLE DAM 15 BONNEVILLE DAM 15 (HOOD RIVER 15)

BONNEVILLE DAM 15

ZANGAR IUNCTION 7.5

YESMOWIT CANYON 7.5 (MT. RAINIER WEST 7.5) MT. RAINIER WEST 7.5

PACKWOOD 15

(PACKWOOD 15)

(GREEN MOUNTAIN 7.5) (MOUNT ADAMS EAST 7.5) (OUTLET FALLS 7.5)

K ROCK 15

(MOUNT ST. HELENS 15) 


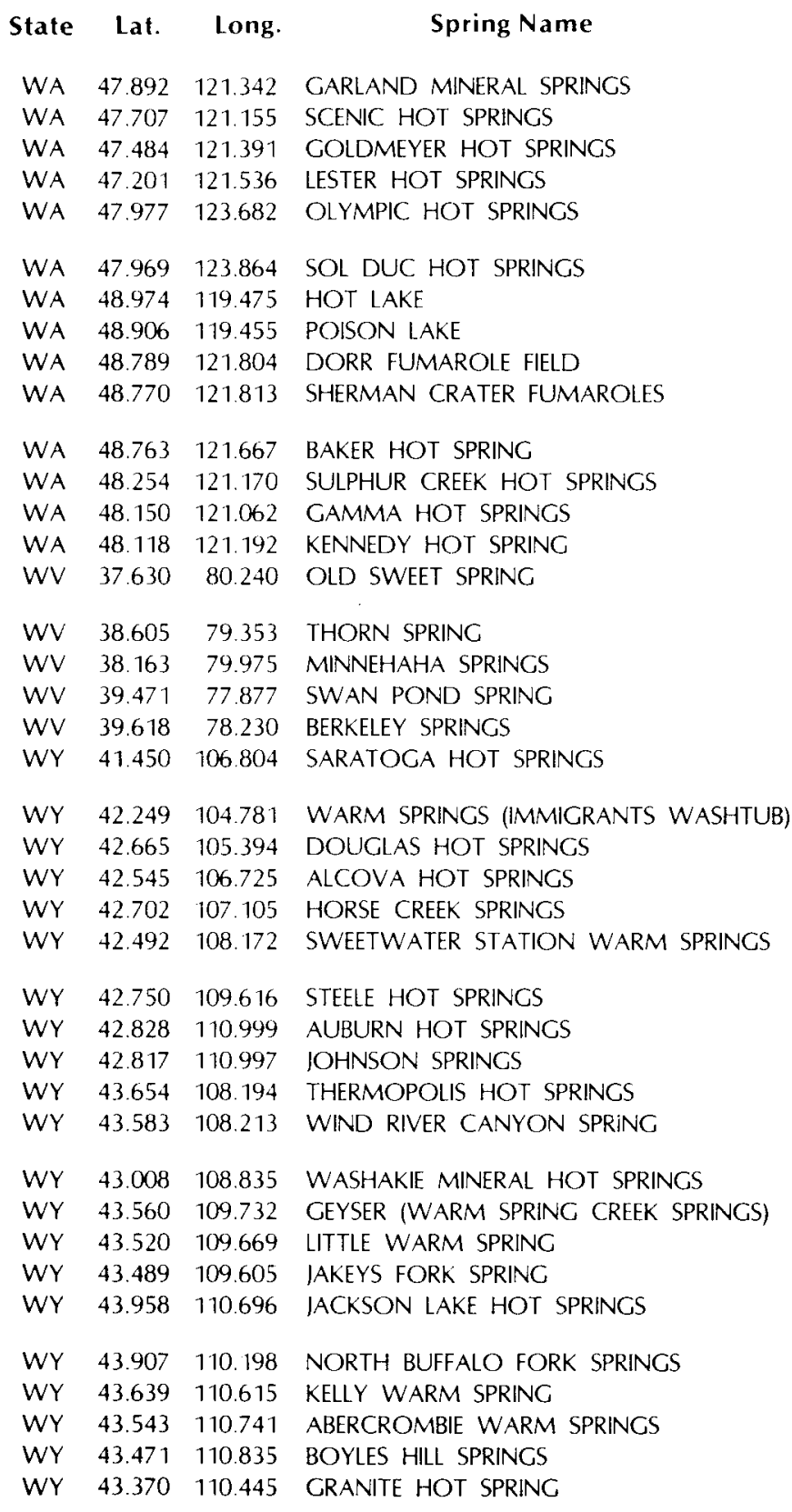

\begin{tabular}{|c|c|c|c|c|c|c|}
\hline \multicolumn{2}{|c|}{$\begin{array}{l}\text { Temperature } \\
{ }^{\circ} \mathrm{F} \quad{ }^{\circ} \mathrm{C}\end{array}$} & $\begin{array}{l}\text { P.P. } \\
492\end{array}$ & $\begin{array}{l}\text { Circ. } \\
790\end{array}$ & \multicolumn{2}{|c|}{ NOAA 1:250,000(AMS)map } & $\begin{array}{l}1: 63360 \text { or } 1: 62500(15 \text {-minute }) \\
\text { or } 1: 24000(7.5 \text {-minute }) \text { quadrangle }\end{array}$ \\
\hline 84 & 29 & 6 & & 1 & WENATCHEE & BLANCA LAKE 7.5 \\
\hline 122 & 50 & 7 & & 2 & WENATCHEE & (SCENIC 7.5) \\
\hline 127 & 53 & 8 & & 3 & WENATCHEE & SNOQUALMIE PASS 15 \\
\hline 120 & 49 & 9 & & 4 & WENATCHEE & GREENWATER 15 \\
\hline 118 & 48 & 3 & & 1 & SEATTLE & MOUNT CARRIE 7.5 \\
\hline 122 & 50 & 2 & & 2 & SEATTLE & BOGACHIEL PEAK 7.5 \\
\hline 122 & 50 & & & 1 & OKANOGAN & OROVILLE 15 \\
\hline 122 & 50 & & & 2 & OKANOGAN & (OROVILLE 15) \\
\hline 194 & 90 & & & 1 & CONCRETE & (MT. BAKER 15) \\
\hline 266 & 130 & & & 2 & CONCRETE & (MT. BAKER 15) \\
\hline 108 & 42 & 1 & 212 & 3 & CONCRETE & MT. SHUKSAN 15 \\
\hline 99 & 37 & 4 & & 4 & CONCRETE & DOWNEY MTN. 7.5 \\
\hline 140 & 60 & & 213 & 5 & CONCRETE & GLACIER PEAK 15 \\
\hline 100 & 38 & 5 & & 6 & CONCRETE & GLACIER PEAK 15 \\
\hline 73 & 23 & 27 & & 1 & BLUEFIELD & ALLEGHANY 7.5 \\
\hline 72 & 22 & 16 & & 1 & CHARLOTTESVILLE & (SUGAR GROVE 7.5) \\
\hline 70 & 21 & 24 & & 2 & CHARLOTTESVILLE & MINNEHAHA SPRINGS 7.5 \\
\hline 72 & 22 & 4 & & 1 & BALTIMORE & (MART!NSBURG 7.5) \\
\hline 72 & 22 & 3 & & 1 & CUMBERLAND & HANCOCK 7.5 \\
\hline 129 & 54 & 115 & & 1 & RAWLINS & SARATOGA 7.5 \\
\hline 70 & 21 & 116 & & 1 & TORRINGTON & WHEATLAND NE 7.5 \\
\hline 86 & 30 & 114 & & 1 & TORRINGTON & CHALK BUTTES 7.5 \\
\hline 129 & 54 & 113 & & 1 & CASPER & (ALCOVA 7.5) \\
\hline 75 & 24 & 112 & & 1 & CASPER & HORSE CREEK SPRINCS 7.5 \\
\hline 90 & 32 & 110 & & 1 & LANDER & HAPPY SPRING 7.5 \\
\hline 102 & 39 & 105 & & 1 & LANDER & FREMONT BUTTE 7.5 \\
\hline 144 & 62 & 103 & 218 & 1 & PRESTON & \\
\hline 115 & 46 & & & 2 & PRESTON & \\
\hline 133 & 56 & 111 & & 1 & THERMOPOLIS & THERMOPOLIS 7.5 \\
\hline 72 & 22 & & & 2 & THERMOPOLIS & WEDDING OF THE WATERS 7.5 \\
\hline 111 & 44 & 108 & & 3 & THERMOPOLIS & ETHETE 7.5 \\
\hline 84 & 29 & 106 & & 1 & THERMOPOLIS & DUBOIS 7.5 \\
\hline 77 & 25 & 107 & & 2 & THERMOPOLIS & (DUBOIS 7.5) \\
\hline 68 & 20 & & & 3 & THERMOPOLIS & (TORREY LAKE 7.5) \\
\hline 162 & 72 & & & 1 & DRICGS & (COLTER BAY 7.5) \\
\hline 113 & 45 & & & 2 & DRICCS & (JOY PEAK 7.5) \\
\hline 81 & 27 & & & 3 & DRICGS & SHADOW MOUNTAIN 7.5 \\
\hline 81 & 27 & & & 4 & DRICGS & GROS VENTRE JUNCTION 7.5 \\
\hline 86 & 30 & & & 5 & DRIGCS & (IACKSON 7.5) \\
\hline 106 & 41 & 102 & 217 & 6 & DRIGCS & GRANITE FALLS 7.5 \\
\hline
\end{tabular}




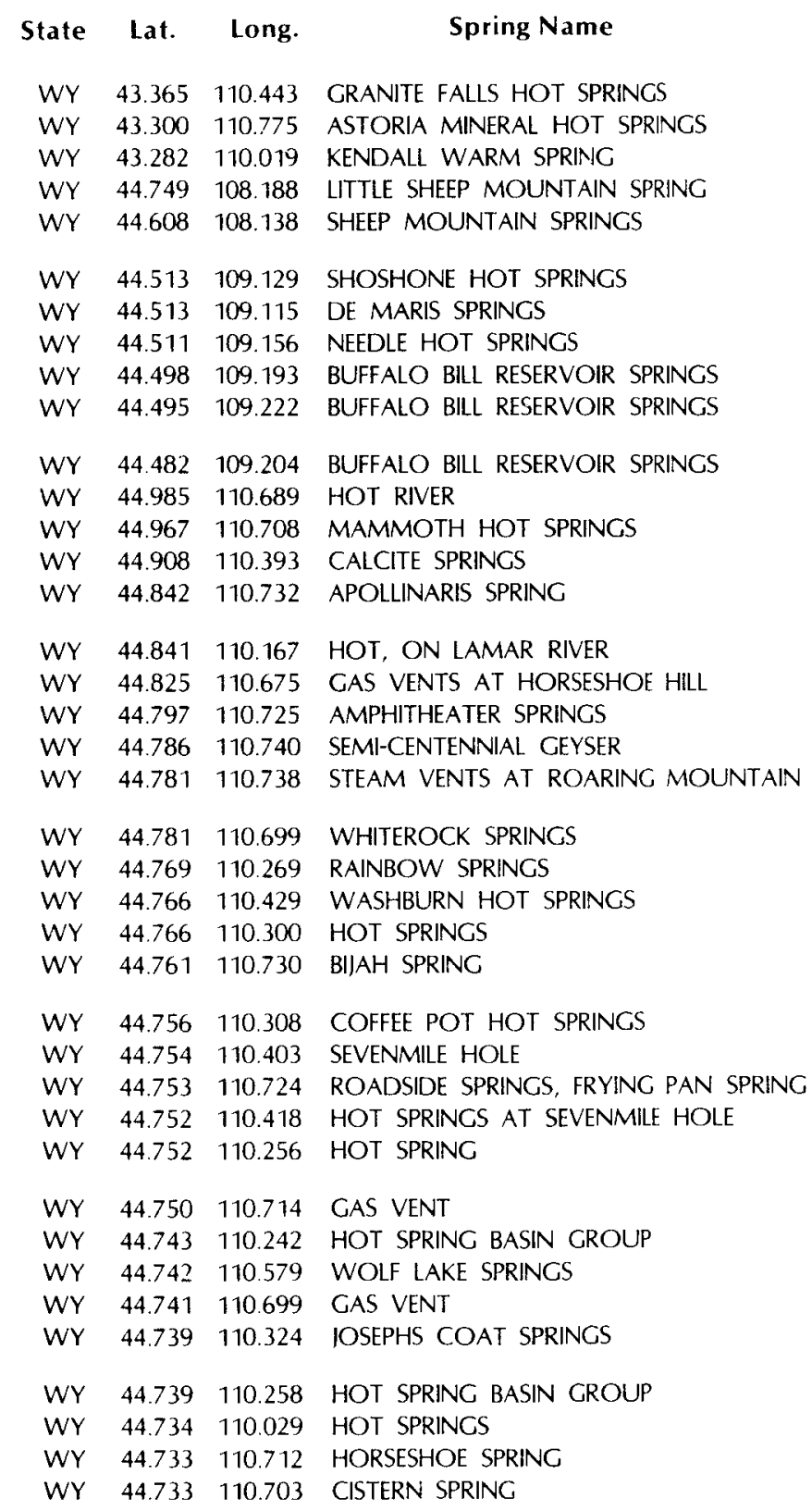

\begin{tabular}{|c|c|c|c|c|c|c|}
\hline $\begin{array}{l}\text { Temp } \\
{ }^{\circ} \mathrm{F}\end{array}$ & ${ }^{\circ} \mathrm{C}$ & $\begin{array}{l}\text { P.P. } \\
492\end{array}$ & $\begin{array}{l}\text { Circ. } \\
790\end{array}$ & NOA & $1: 250,000(A M S)$ map & $\begin{array}{l}1: 63360 \text { or } 1: 62500(15 \text {-minute }) \\
\text { or } 1: 24000(7.5 \text {-minute) quadrangle }\end{array}$ \\
\hline 112 & 45 & & & 7 & DRIGGS & (GRANITE FALLS 7.5) \\
\hline 99 & 37 & 101 & & 8 & DRICGS & MUNCER MOUNTAIN 7.5 \\
\hline 86 & 30 & 104 & & 9 & DRIGCS & KLONDIKE HILL 7.5 \\
\hline 68 & 20 & 98 & & 1 & CODY & (SPENCE 7.5) \\
\hline 70 & 21 & 99 & & 2 & CODY & SHEEP CANYON 7.5 \\
\hline 95 & 35 & & & 1 & CODY & (CODY 15) \\
\hline 97 & 36 & 97 & & 2 & CODY & CODY 15 \\
\hline 75 & 24 & & & 3 & CODY & (CODY 15) \\
\hline W & W & & & 4 & CODY & (DEVILS TOOTH 15) \\
\hline W & W & & & 5 & CODY & (DEVILS TOOTH 15) \\
\hline W & W & & & 6 & CODY & (DEVILS TOOTH 15) \\
\hline 136 & 58 & 1 & 215 & 1 & ASHTON & MAMMOTH 15 \\
\hline 163 & 73 & 2 & 215 & 2 & ASHTON & MAMMOTH 15 \\
\hline 201 & 94 & $65 \mathrm{~A}$ & 215 & 3 & ASHTON & TOWER JUNCTION 15 \\
\hline$H$ & $\mathrm{H}$ & & 215 & 4 & ASHTON & MAMMOTH 15 \\
\hline W & W & 66 & 215 & 5 & ASHTON & (ABIATHAR PEAK 15) \\
\hline $\mathrm{H}$ & $\mathrm{H}$ & & 215 & 6 & ASHTON & MAMMOTH 15 \\
\hline 196 & 91 & 7 & 215 & 7 & ASHTON & MAMMOTH 15 \\
\hline 198 & 92 & $7 \mathrm{~B}$ & 215 & 8 & ASHTON & MAMMOTH 15 \\
\hline $\mathrm{H}$ & $\mathrm{H}$ & & 215 & 9 & ASHTON & MAMMOTH 15 \\
\hline 144 & 62 & 8 & 215 & 10 & ASHTON & MAMMOTH 15 \\
\hline 190 & 88 & & 215 & 11 & ASHTON & TOWER JUNCTION 15 \\
\hline 196 & 91 & 50 & 215 & 12 & ASHTON & TOWER IUNCTION 15 \\
\hline $\mathrm{H}$ & $\mathrm{H}$ & & 215 & 13 & ASHTON & TOWER JUNCTION 15 \\
\hline 198 & 92 & 9 & 215 & 14 & ASHTON & MAMMOTH 15 \\
\hline 196 & 91 & 73 & 215 & 15 & ASHTON & TOWER JUNCTION 15 \\
\hline 154 & 68 & & 215 & 16 & ASHTON & (TOWER JUNCTION 15) \\
\hline 158 & 70 & $9 \mathrm{~A}$ & 215 & 17 & ASHTON & MAMMOTH 15 \\
\hline 189 & 87 & 52 & 215 & 18 & ASHTON & TOWER JUNCTION 15 \\
\hline $\mathrm{H}$ & $H$ & & 215 & 19 & ASHTON & TOWER IUNCTION 15 \\
\hline $\mathrm{H}$ & $\mathrm{H}$ & & 215 & 20 & ASHTON & MAMMOTH 15 \\
\hline 198 & 92 & 75 & 215 & 21 & ASHTON & PELICAN CONE 15 \\
\hline 91 & 33 & & 215 & 22 & ASHTON & (NORRIS JUNCTION 15) \\
\hline $\mathrm{H}$ & $H$ & & 215 & 23 & ASHTON & NORRIS JUNCTION 15 \\
\hline 199 & 93 & 72 & 215 & 24 & ASHTON & CANYON VILLACE 15 \\
\hline 153 & 67 & 75 & 215 & 25 & ASHTON & CANYON VILAGE 15 \\
\hline W & w & 76 & 215 & 26 & ASHTON & PELICAN CONE 15 \\
\hline 187 & 86 & & 215 & 27 & ASHTON & (NORRIS JUNCTION 15) \\
\hline 199 & 93 & & 215 & 28 & ASHTON & (NORRIS JUNCTION 15) \\
\hline 192 & 89 & & 215 & 29 & ASHTON & NORRIS JUNCTION 15 \\
\hline
\end{tabular}




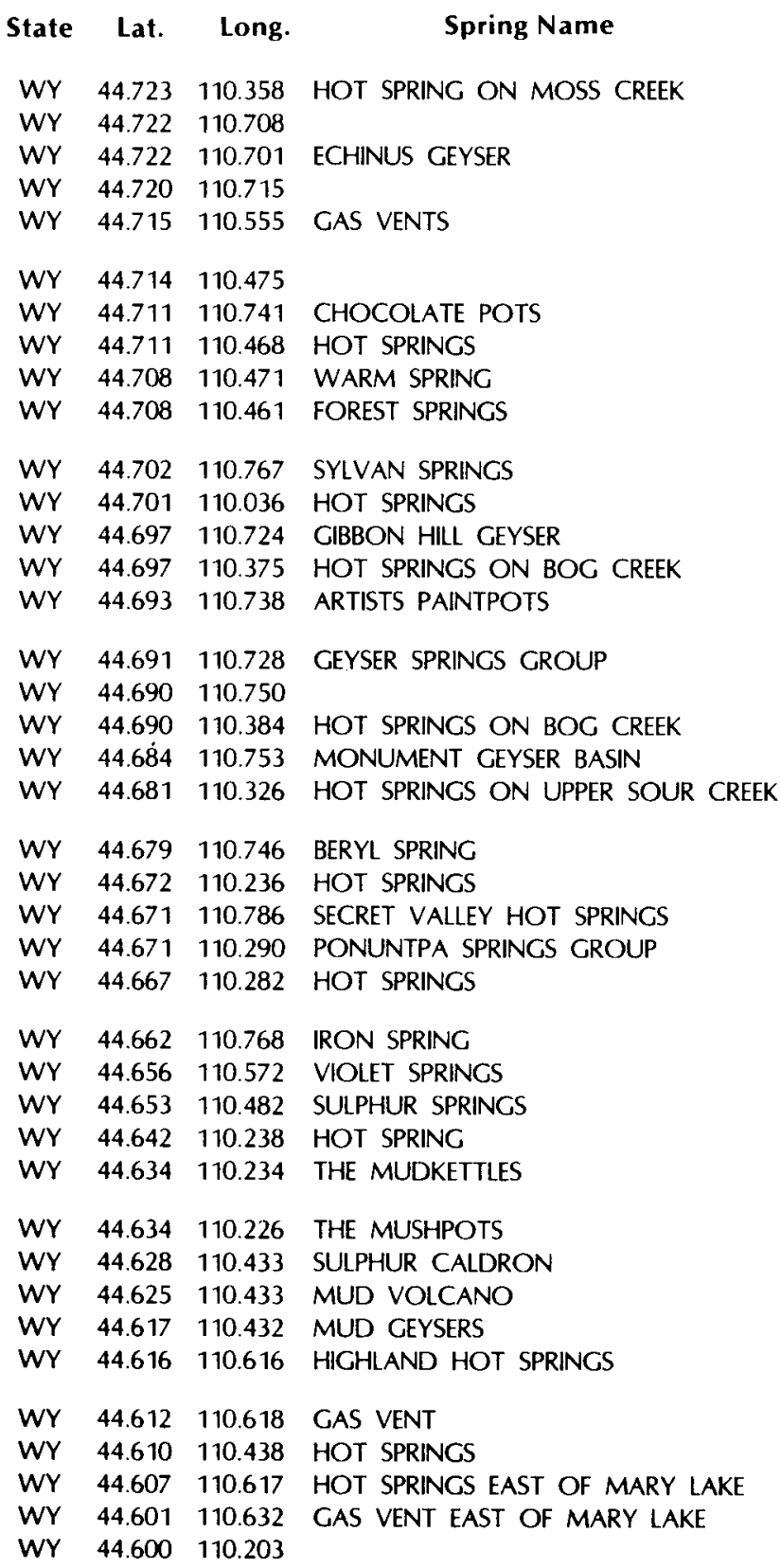

\begin{tabular}{|c|c|c|c|c|c|}
\hline \multicolumn{2}{|c|}{ Temperature } & \multirow{2}{*}{$\begin{array}{l}\text { P.P. } \\
492\end{array}$} & \multirow{2}{*}{$\begin{array}{c}\text { Circ. } \\
790\end{array}$} & \multicolumn{2}{|c|}{ NOAA $1: 250,000$ (AMS)map } \\
\hline${ }^{\circ} \mathbf{F}$ & ${ }^{\circ} \mathrm{C}$ & & & & \\
\hline 115 & 46 & 78 & 215 & 30 & ASHTON \\
\hline 201 & 94 & & 215 & 31 & ASHTON \\
\hline 185 & 85 & 11 & 215 & 32 & ASHTON \\
\hline 144 & 62 & & 215 & 33 & ASHTON \\
\hline $\mathrm{H}$ & $\mathrm{H}$ & & 215 & 34 & ASHTON \\
\hline 167 & 75 & & 215 & 35 & ASHTON \\
\hline 124 & 51 & & 215 & 36 & ASHTON \\
\hline $\mathrm{H}$ & $\mathrm{H}$ & & 215 & 37 & ASHTON \\
\hline w & W & & 215 & 38 & ASHTON \\
\hline $\mathrm{H}$ & $\mathrm{H}$ & 54 & 215 & 39 & ASHTON \\
\hline 192 & 89 & 12 & 215 & 40 & ASHTON \\
\hline W & W & 77 & 215 & 41 & ASHTON \\
\hline 199 & 93 & 13 & 215 & 42 & ASHTON \\
\hline 185 & 85 & 79 & 215 & 43 & ASHTON \\
\hline 199 & 93 & 14 & 215 & 44 & ASHTON \\
\hline 201 & 94 & 15 & 215 & 45 & ASHTON \\
\hline 187 & 86 & & 215 & 46 & ASHTON \\
\hline 199 & 93 & 79 & 215 & 47 & ASHTON \\
\hline 197 & 92 & 16 & 215 & 48 & ASHTON \\
\hline 194 & 90 & & 215 & 49 & ASHTON \\
\hline 198 & 92 & $16 \mathrm{~A}$ & 215 & 50 & ASHTON \\
\hline$H$ & $\mathrm{H}$ & & 215 & 51 & ASHTON \\
\hline 95 & 35 & & 215 & 52 & ASHTON \\
\hline 180 & 82 & 83 & 215 & 53 & ASHTON \\
\hline $\mathrm{H}$ & $H$ & & 215 & 54 & ASHTON \\
\hline 86 & 30 & & 215 & 55 & ASHTON \\
\hline $\mathrm{H}$ & $\mathrm{H}$ & 56 & 215 & 56 & ASHTON \\
\hline 194 & 89 & 61 & 215 & 57 & ASHTON \\
\hline $\mathrm{H}$ & $\mathrm{H}$ & & 215 & 58 & ASHTON \\
\hline H & $\mathrm{H}$ & 87 & 215 & 59 & ASHTON \\
\hline $\mathrm{H}$ & H & 88 & 215 & 60 & ASHTON \\
\hline 190 & 88 & $61 \mathrm{D}$ & 215 & 61 & ASHTON \\
\hline 131 & 55 & $61 C$ & 215 & 62 & ASHTON \\
\hline $\mathrm{H}$ & $\mathrm{H}$ & $61 \mathrm{C}$ & 215 & 63 & ASHTON \\
\hline $\mathrm{H}$ & $H$ & 57 & 215 & 64 & ASHTON \\
\hline $\mathrm{H}$ & $\mathrm{H}$ & & 215 & 65 & ASHTON \\
\hline $\mathrm{H}$ & H & & 215 & 66 & ASHTON \\
\hline$H$ & $H$ & 59 & 215 & 67 & ASHTON \\
\hline$H$ & $H$ & & 215 & 68 & ASHTON \\
\hline 95 & 35 & & 215 & 69 & ASHTON \\
\hline
\end{tabular}

1:63360 or $1: 62500(15$-minute $)$ or 1:24000 (7.5-minute) quadrangle

CANYON VILLACE 15 NORRIS JUNCTION 15 NORRIS JUNCTION 15 NORRIS JUNCTION 15 NORRIS IUNCTION 15

CANYON VILLAGE 15 NORRIS IUNCTION 15 CANYON VILLACE 15 CANYON VILLAGE 15 CANYON VILLAGE 15

MADISON JUNCTION 15 PELICAN CONE 15 NORRIS JUNCTION 15 CANYON VILLACE 15 NORRIS JUNCTION 15

NORRIS JUNCTION 15 NORRIS JUNCTION 15 CANYON VILLACE 15 MADISON JUNCTION 15 CANYON VILLAGE 15

NORRIS JUNCTION 15 PELICAN CONE 15

(MADISON IUNCTION 15)

CANYON VILLAGE 15

CANYON VILLAGE 15

MADISON IUNCTION 15

NORRIS JUNCTION 15

CANYON VILLACE 15

PELICAN CONE 15

PELICAN CONE 15

PELICAN CONE 15 CANYON VILLAGE 15 CANYON VILLACE 15 CANYON VILLACE 15 RIS JUNCTION 15

NORRIS JUNCTION 15 CANYON VILLAGE 15 NORRIS JUNCTION 15 NORRIS JUNCTION 15 PELICAN CONE 15 


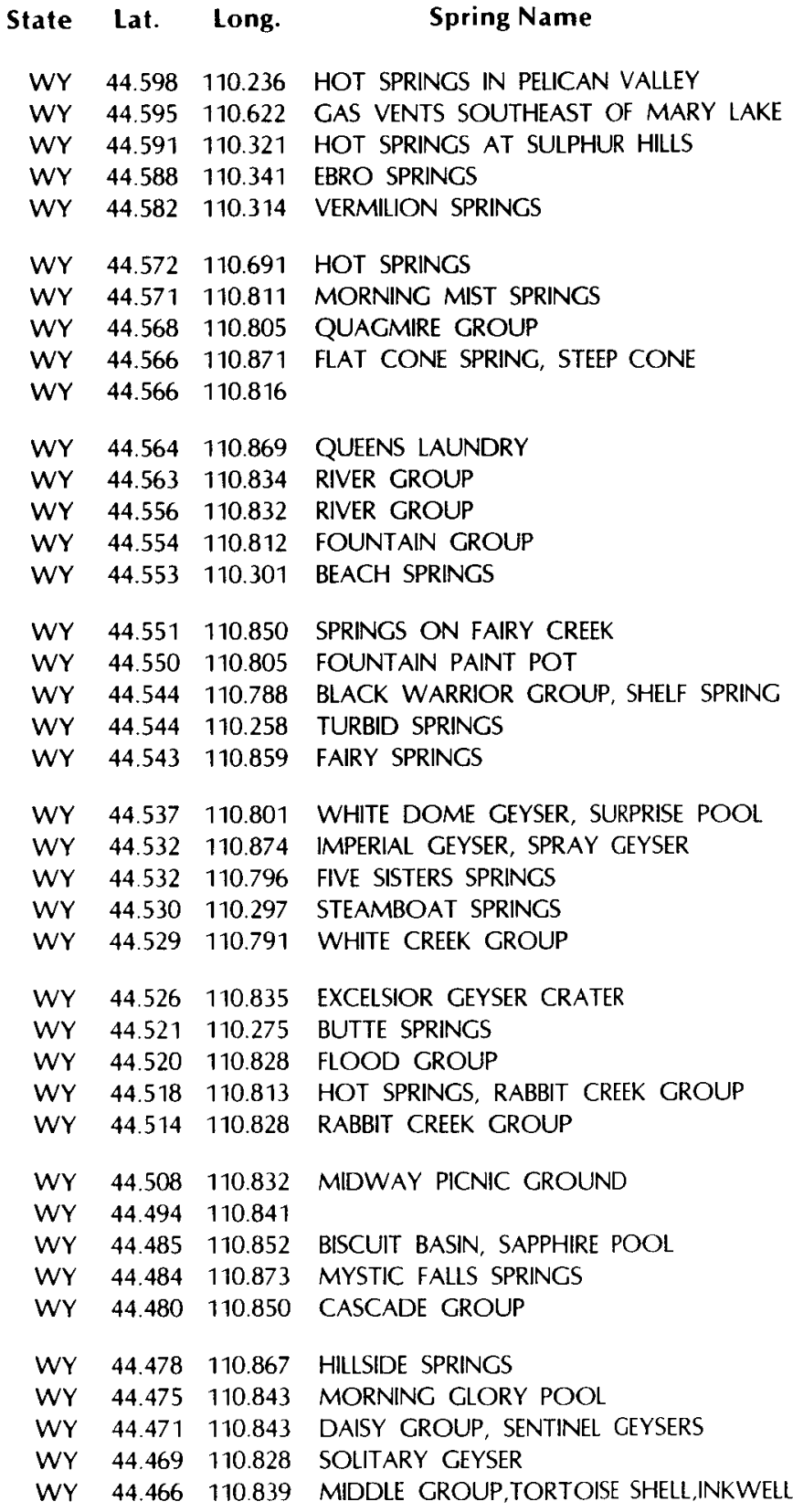

$\begin{array}{llll}\text { WY } & 44.598 & 110.236 & \text { HOT SPRINCS IN PELICAN VALLEY }\end{array}$

WY $44.595 \quad 110.622$ GAS VENTS SOUTHEAST OF MARY LAKE

WY $44.591 \quad 110.321$ HOT SPRINCS AT SULPHUR HILLS

WY $44.588 \quad 110.341 \quad$ EBRO SPRINGS

WY $\quad 44.582 \quad 110.314 \quad$ VERMILION SPRINCS

WY $44.572 \quad 110.691 \quad$ HOT SPRINCS

WY $\quad 44.571 \quad 110.811$ MORNING MIST SPRINCS

WY $44.568 \quad 110.805$ QUACMIRE GROUP

WY $44.566 \quad 110.871$ FLAT CONE SPRING, STEEP CONE

WY $\quad 44.566 \quad 110.816$

WY $\quad 44.564 \quad 110.869$ QUEENS LAUNDRY

WY $\quad 44.563 \quad 110.834 \quad$ RIVER GROUP

WY $44.556 \quad 110.832$ RIVER GROUP

WY $\quad 44.554 \quad 110.812$ FOUNTAIN GROUP

WY $44.553 \quad 110.301 \quad$ BEACH SPRINCS

WY $44.551 \quad 110.850 \quad$ SPRINCS ON FAIRY CREEK

WY $\quad 44.550 \quad 110.805$ FOUNTAIN PAINT POT

WY $44.544 \quad 110788$ BLACK WARRIOR GROUP, SHELF SPRINC

WY $\quad 44.544 \quad 110.258$ TURBID SPRINCS

WY $44.543 \quad 110.859$ FAIRY SPRINCS

WY $44.537 \quad 110.801$ WHITE DOME GEYSER, SURPRISE POOL

WY $44.532 \quad 110.874$ IMPERIAL GEYSER, SPRAY GEYSER

WY $44.532 \quad 110.796 \quad$ FIVE SISTERS SPRINCS

WY $44.530 \quad 110.297 \quad$ STEAMBOAT SPRINCS

WY $44.529 \quad 110.791 \quad$ WHITE CREEK GROUP

WY $44.526 \quad 110.835$ EXCELSIOR GEYSER CRATER

WY $44.521 \quad 110.275 \quad$ BUTTE SPRINGS

WY $44.520 \quad 110828 \quad$ FLOOD CROUP

WY $44.518 \quad 110.813$ HOT SPRINCS, RABBIT CREEK CROUP

WY $44.514 \quad 110.828$ RABBIT CREEK GROUP

WY $44.508 \quad 110.832$ MIDWAY PICNIC GROUND

WY $\quad 44.494 \quad 110.841$

WY $44.485 \quad 110.852$ BISCUIT BASIN, SAPPHIRE POOL

WY $44.484 \quad 110.873$ MYSTIC FALLS SPRINGS

WY $\quad 44.480 \quad 110.850$ CASCADE GROUP

WY $44.478 \quad 110.867 \quad$ HILLSIDE SPRINCS

WY $44.475 \quad 110.843$ MORNING GLORY POOL

WY 44471110843 DAISY GROUP, SENTINEL CEYSERS

WY $44.469 \quad 110.828$ SOLITARY GEYSER

WY $\quad 44.466 \quad 110.839$ MIDDLE GROUP,TORTOISE SHELL,INKWELL

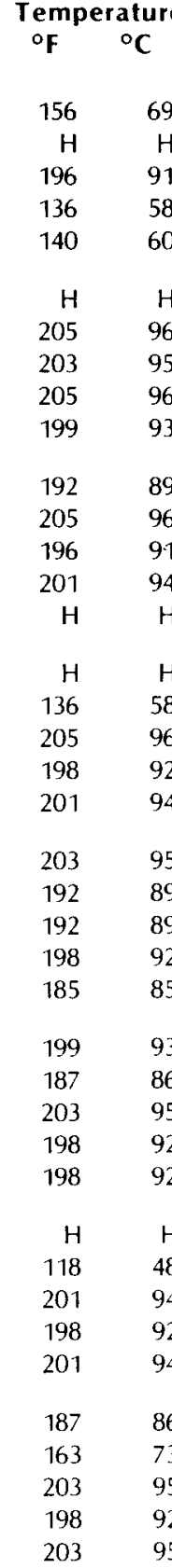

P.P. Circ. NOAA 1:250,000(AMS)map 492790

$\begin{array}{lll} & 215 & 70 \\ & 215 & 71 \\ 89 & 215 & 72 \\ 90 & 215 & 73 \\ 91 & 215 & 74 \\ & & \\ 27 & 215 & 75 \\ 19 & 215 & 76 \\ & 215 & 77 \\ & 215 & 78 \\ & 215 & 79 \\ 17 & 215 & 80 \\ 18 & 215 & 81 \\ & 215 & 82 \\ & 215 & 83 \\ 93 & 215 & 84\end{array}$

ASHTON

ASHTON

ASHTON

ASHTON

ASHTON

ASHTON

ASHTON

ASHTON

ASHTON

ASHTON

ASHTON

ASHTON

ASHTON

ASHTON

$\begin{array}{llll}93 & 215 & 84 & \text { ASHTON }\end{array}$

$\begin{array}{lll}215 & 85 & \text { ASHTON }\end{array}$

$\begin{array}{llll}21 & 215 & 86 & \text { ASHTON }\end{array}$

$\begin{array}{lll}215 & 87 & \text { ASHTON }\end{array}$

$\begin{array}{llll}94 & 215 & 88 & \text { ASHTON }\end{array}$

$\begin{array}{llll}20 & 215 & 89 & \text { ASHTON }\end{array}$

$\begin{array}{llll}22 & 215 & 90 & \text { ASHTON }\end{array}$

24A 21591 ASHTON

21592 ASHTON

$\begin{array}{llll}95 & 215 & 93 & \text { ASHTON }\end{array}$

ASHTON

$\begin{array}{llll}25 & 215 & 95 & \text { ASHTON }\end{array}$

$\begin{array}{llll}96 & 215 & 96 & \text { ASHTON }\end{array}$

$\begin{array}{llll}26 & 215 & 97 & \text { ASHTON }\end{array}$

$\begin{array}{lll}26 A & 215 & 98 \\ \end{array}$

ASHTON

ASHTON

$29 \quad 215 \quad 102$ ASHTON

215103 ASHTON

215104 ASHTON

$\begin{array}{lll}215 & 105 & \text { ASHTON }\end{array}$

$\begin{array}{llll}30 \mathrm{~A} & 215 & 106 & \text { ASHTON }\end{array}$

$\begin{array}{llll}31 & 215 & 107 & \text { ASHTON }\end{array}$

32B $215 \quad 108$ ASHTON
$1: 63360$ or $1: 62500$ (15-minute)

or 1:24000 (7.5-minute) quadrangle

PELICAN CONE 15

NORRIS JUNCTION 15

CANYON VILLAGE 15

CANYON VILLAGE 15

CANYON VILLAGE 15

NORRIS IUNCTION 15

MADISON JUNCTION 15

MADISON IUNCTION 15

MADISON IUNCTION 15

MADISON IUNCTION 15

MADISON IUNCTION 15

MADISON JUNCTION 15

MADISON JUNCTION 15

CANYON VILLAGE 15

MADISON JUNCTION 15

MADISON IUNCTION 15

CANYON VILLACE 15

MADISON JUNCTION 15

MADISON IUNCTION 15

MADISON JUNCTION 15

MADISON JUNCTION 15

CANYON VILLAGE 15

(MADISON JUNCTION 15)

MADISON IUNCTION 15

CANYON VILLACE 15

MADISON IUNCTION 15

MADISON IUNCTION 15

MADISON IUNCTION 15

MADISON JUNCTION 15)

(OLD FAITHFUL. 15)

OLD FAITHFUL 15

OLD FAITHFUL 15

(OLD FAITHFUL 15)

OLD FAITHFUL 15

OLD FAITHFUL 15

OLD FAITHEL 15

OLD FAITHFUL 15

OLD FAITHFUL 15 


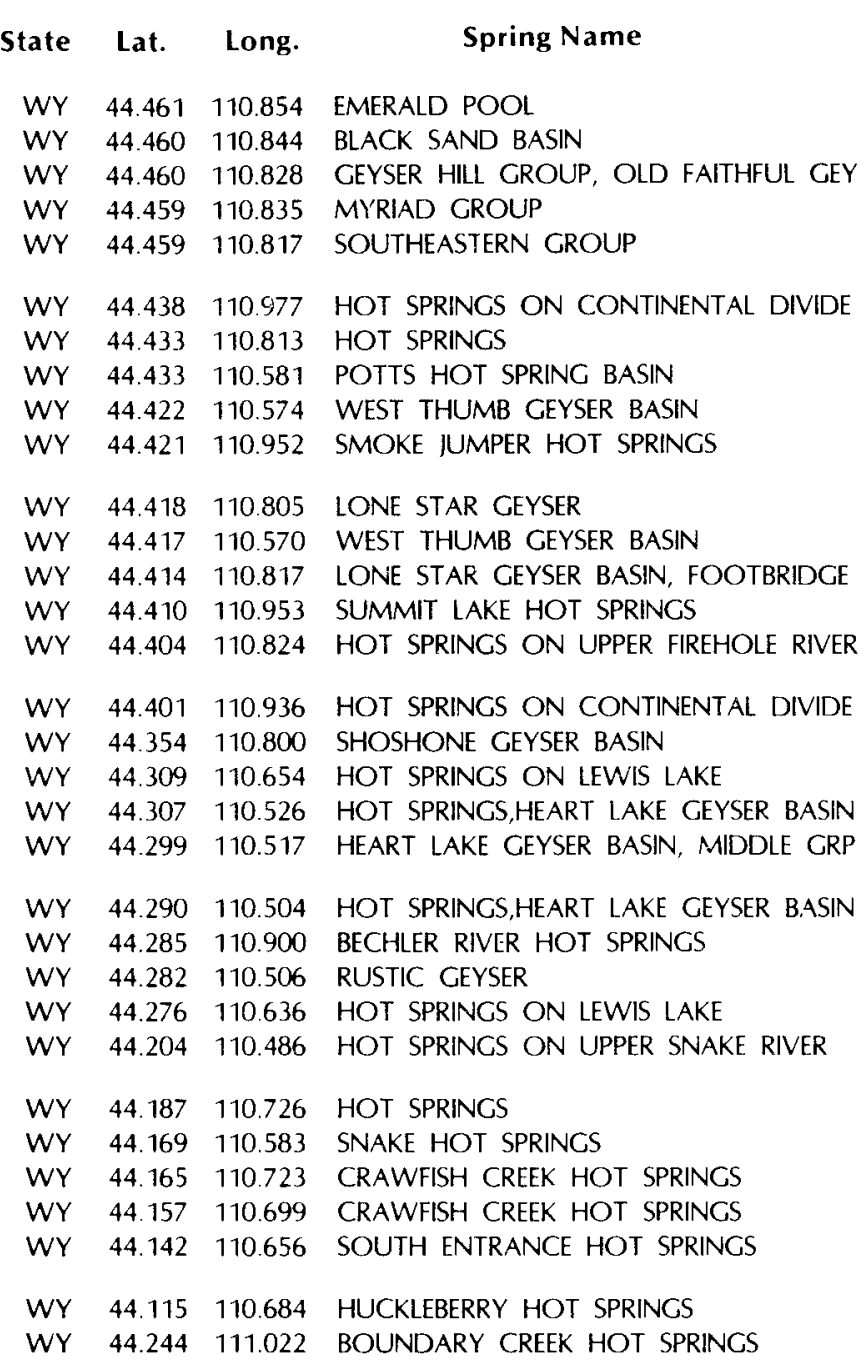

\begin{tabular}{|c|c|}
\hline \multicolumn{2}{|c|}{$\begin{array}{l}\text { Temperature } \\
{ }^{\circ} \mathbf{F}{ }^{\circ} \mathbf{C}\end{array}$} \\
\hline 158 & 70 \\
\hline 201 & 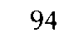 \\
\hline 203 & 0 \\
\hline 201 & \\
\hline 198 & \\
\hline $\mathrm{H}$ & \\
\hline $\mathrm{H}$ & \\
\hline 203 & \\
\hline 196 & \\
\hline 198 & \\
\hline 199 & \\
\hline 203 & \\
\hline 183 & \\
\hline 162 & \\
\hline $\mathrm{H}$ & \\
\hline 189 & \\
\hline 203 & \\
\hline 199 & 9 \\
\hline 201 & \\
\hline 174 & \\
\hline 201 & \\
\hline 194 & \\
\hline 199 & \\
\hline 154 & \\
\hline 167 & \\
\hline 142 & \\
\hline 136 & \\
\hline 138 & \\
\hline 136 & \\
\hline 156 & \\
\hline B & \\
\hline
\end{tabular}

P.P. Circ. NOAA $1: 250,000$ (AMS)map $492 \quad 790$

$\begin{array}{lll}33 & 215 & 110\end{array}$

$\begin{array}{llll}33 & 215 & 111 & \text { ASHTON }\end{array}$

$\begin{array}{llll}34 & 215 & 112 & \text { ASHTON }\end{array}$ 215113 ASHTON 215114 ASHTON

215115 ASHTON

$63215 \quad 117$ ASHTON $\begin{array}{lll}215 & 118 & \text { ASHTON } \\ 215 & 119 & \text { ASHTON }\end{array}$

$\begin{array}{llll}37 & 215 & 120 & \text { ASHTON }\end{array}$

$\begin{array}{llll}64 & 215 & 121 & \text { ASHTON }\end{array}$

$\begin{array}{lll}215 & 122 & \text { ASHTON }\end{array}$

$\begin{array}{llll}35 & 215 & 123 & \text { ASHTON }\end{array}$ $\begin{array}{lll}215 & 124 & \text { ASHTON }\end{array}$

$\begin{array}{llll}36 & 215 & 125 & \text { ASHTON }\end{array}$

$\begin{array}{llll}38 & 215 & 126 & \text { ASHTON }\end{array}$

$\begin{array}{llll}42 & 215 & 127 & \text { ASHTON }\end{array}$ $215 \quad 128$ ASHTON $\begin{array}{ll}215 & 129 \\ & \text { ASHTON }\end{array}$

215130 ASHTON

$\begin{array}{llll}39 & 215 & 131 & \text { ASHTON }\end{array}$

$\begin{array}{llll}45 & 215 & 132 & \text { ASHTON }\end{array}$

$\begin{array}{llll}43 & 215 & 133 & \text { ASHTON }\end{array}$

$\begin{array}{llll}48 & 215 & 134 & \text { ASHTON }\end{array}$

215135 ASHTON

4215136 ASHTON ASHTON

$\begin{array}{lll}46 & 215 \quad 139 \quad \text { ASHTON }\end{array}$ $\begin{array}{rrrr}100 & 216 & 140 & \text { ASHTON } \\ 215 & 1 & \text { ASHTON }\end{array}$
$1: 63360$ or $1: 62500$ (15-minute) or 1:24000 (7.5-minute) quadrangle

OLD FAITHFUL 15

OLD FAITHFUL 15

OLD FAITHFUL 15

OLD FAITHFUL 15

OLD FAITHFUI 15

OLD FAITHFUL 15 OLD FAITHFUL 15 WEST THUMB 15 WEST THUMB 15 OLD FAITHFUL 15

OLD FAITHFUL 15 WEST THUMB 15 OLD FAITHFUL 15 OLD FAITHFUL 15 OLD FAITHFUI 15

OLD FAITHFUL 15 OLD FAITHFUL 15 WEST THUMB 15 WEST THUMB 15 WEST THUMB 15

WEST THUMB 15 OLD FAITHFUL 15

WEST THUMB 15

WEST THUMB 15

MOUNT HANCOCK 15

HUCKLEBERRY MOUNTAIN 15 HUCKLEBERRY MOUNTAIN 15 HUCKLEBERRY MOUNTAIN 15 HUCKLEBERRY MOUNTAIN 15 HUCKLEBERRY MOUNTAIN 15

HUCKLEBERRY MOUNTAIN 15 WARM RIVER BUTTE 15 
CA ABBOTT MINE

WY ABERCROMBIE WARM SPRINCS

UT ABRAHAM HOT SPRINCS

CA AETNA SPRINGS

AZ AGUA CALIENTE

AZ AGUA CALIENTE SPRING

CA. AGUA CALIENTE SPRING

AZ AGUA CALIENTE SPRINGS

CA AGUA CALIENTE SPRINGS

CA AGUA TIBIA SPRINC

HI AKINS SPRINC

CA ALAMEDA WARM SPRINCS

NM ALAMOS SPRINC

WY ALCOVA HOT SPRINGS

MT ALHAMBRA HOT SPRINGS

NV ALKALI HOT SPRINC
NM ALIEN SPRINGS

NV ALLEN SPRINCS

D ALPINE WARM SPRINC

OR ALTNOW HOT SPRINC

$\checkmark A$ ALUM SPRINGS

OR ALVORD HOT SPRINCS

CA AMEDEE HOT SPRINGS

$W Y$ AMPHITHEATER SPRINCS

MT ANACONDA HOT SPRINCS

NV ANAHO ISLAND SPRINC

CA ANDERSON SPRINCS

MT ANDERSON SPRINCS

MT ANDERSONS PASTURE SPRINGS

OR ANNIE SPRINC

OR ANTELOPE HOT SPRINGS

AZ ANTELOPE SPRING

CO ANTELOPE SPRING

NM APACHE TEJO WARM SPRING

MT APEX WARM SPRING

WY APOUINARIS SPRING

NM ARAGON SPRINGS

ID ARLING HOT SPRINC

CA ARROWHEAD SPRINGS

NM ARSENIC SPRING

ID ARTESIAN CITY HOT SPRINCS

WY ARTISTS PAINTPOTS

NV ASH CREEK SPRING

NV ASH MEADOWS SPRING
ASH SPRINC

NV ASH SPRINGS

NV ASH TREE SPRING

ID ASHTON WARM SPRINC

AZ ASTIN SPRING

WY ASTORIA MINERAL HOT SPRINGS

ID ATLANTA HOT SPRINGS

WY AUBURN HOT SPRINGS

OR AUSTIN HOT SPRINGS

MT AVON WARM SPRINC

CA BAD CREEK SPRING

ID BADLEY WARM SPRINC

OR BAGBY HOT SPRINCS

AK BAILEY HOT SPRINC

CA BAINTER SPRING

WA BAKER HOT SPRING

UT BAKER HOT SPRINGS

CA BAKER SODA SPRING

OR BAKER SPRING

NV BALTAZOR HOT SPRINC

BANBURY HOT SPRING

AK BARANOF WARM SPRINGS

CA BARE RANCH SPRINC

GA BARKER SPRING

AK BARNES LAKE HOT SPRINGS

BARNEY HOT SPRINGS

BARRONS HOT SPRING

OR BARRY RANCH HOT SPRINCS

ID BARTH HOT SPRINCS

NV BARTHOLOMAE HOT SPRINC

$\begin{array}{ll}N V & \text { BARTHOLOMAE HOT } \\ \text { NV BARTINE HOT SPRINGS }\end{array}$

CA BARTLETT SPRINGS

ID BASIN CREEK HOT SPRINC

OR BASQUE SPRINC

ID BASSET HOT SPRING

CA BASSETT HOT SPRINCS

ID BAT HOT SPRING

ID BATTIE CREEK HOT SPRINCS

ID BAUMGARTNER HOT SPRING

WY BEACH SPRINGS

OR BEAN HOT SPRING

MT BEAR CREEK SPRINGS

ID BEAR LAKE HOT SPRINCS

ID BEAR VALLEY HOT SPRINC

ID BEARDSLEY HOT SPRINGS

MT BEARMOUTH WARM SPRING 1

\section{State Spring name}

MT BEARMOUTH WARM SPRING 2

NV BEATTY MUNICIPAL SPRINC

MT BEAVERHEAD ROCK SPRING

WY BECHLER RIVER HOT SPRINCS

UT BECKS HOT SPRING

MT BEDFORD HOT SPRINCS

OR BELKNAP SPRINCS

AK BELL ISLAND HOT SPRINGS

ID BELVIDERE HOT SPRING

NV BENNETT SPRINGS

CA BENTON HOT SPRINCS

NV BEOWAWE HOT SPRINCS

WV BERKELEY SPRINCS

UT BERRY SPRINGS

CA BERTRAND RANCH SPRINCS

WY BERYL SPRING

OR BEULAH HOT SPRINCS

CA BIC BEND HOT SPRINCS

NV BIC BLUE (A) SPRINC

NV BIC BLUE SPRING

NV BIC BLUE SPRINCS

AR BIC CHALYBEATE SPRINC

ID BIC CREEK HOT SPRINCS

ID BIG EIGHTMILE CREEK WARM SPRINC

OR BIG HOT SPRING

UT BIC MAPLE SPRINC

FL BIC SALT SPRING

ID BIC SMOKEY HOT SPRING

CA BIC SODA SPRINC

ID BIC SPRING

NV BIC SPRINC

NV BIC WARM SPRING

MT BIC WARM SPRINGS

UT BIC WARM SPRINGS

OR BICELOW HOT SPRINGS

WY BIJAH SPRING

ID BILLY HOT SPRINC

$\begin{array}{ll}\text { OR } & \text { BILLY HOT SPRINC } \\ \text { BINGHAM SPRINCS }\end{array}$

UT BIRD ISLAND

CO BIRDSIE WARM SPRINC

WY BISCUIT BASIN

NV BLACK ROCK HOT SPRINC

WY BLACK SAND BASIN

WY BLACK WARRIOR GROUP

ID BLACKFOOT RESERVOIR WARM SPRING 
State Spring name

ID BLACKFOOT RIVER WARM SPRING

CA BLANEY MEADOWS HOT SPRINCS

CA BLANK SPRINC

ID BLM WELL

NV BLOSSOM HOT SPRINGS

UT BLUE CREEK SPRING

NV BLUE EAGLE SPRINCS

MT BLUE JOINT HOT SPRING 1

MT BLUE JOINT HOT SPRINCS 2

UT BLUE LAKE SPRING

OR BLUE MOUNTAIN HOT SPRINCS

NV BLUE POINT SPRINC

NV BOG HOT SPRINGS

CA BOCUS SODA SPRINGS

ID BOLING SPRINCS

NV BOLLING SPRINGS

CA BOILING SPRINGS LAKE

VA BOLAR SPRING

NV BOLE SPRING

ID BONNEVILLE HOT SPRINCS

OR BORAX LAKE

NV BORAX SPRING

CA BORON SPRINC

UT BOTHWELL WARM SPRINGS

ID BOULDER CREEK SPRING

MT BOULDER HOT SPRINGS

WY BOUNDARY CREEK HOT SPRINCS

NV BOWERS MANSION HOT SPRINC

WY BOYLES HILL SPRINCS

MT BOZEMAN HOT SPRINCS

AK BRADFIELD CANAL HOT SPRINCS

NV BRADYS HOT SPRINCS

VA BRACG SPRING

OR BREITENBUSH HOT SPRINCS

ID BRIDGE HOT SPRINGS

MT BRIDGER CANYON WARM SPRING

OR BRISBOIS RANCH SPRINGS

MT BROADWATER HOT SPRINGS

ID BROCKMAN CREEK HOT SPRINC

CA BROCKWAY HOT SPRINCS

NV BROOKS SPRINC

MT BROOKS WARM SPRINC

ID BROWN CREEK HOT SPRING

CO BROWNS CANYON WARM SPRING

CO BROWNS GROTTO WARM SPRINC

\section{Spring name}

NV BROWNS SPRINC

MT BROWNS SPRINCS

NV BRUFFEYS HOT SPRINCS

BRUNEAU HOT SPRING

BUCKAROO HOT SPRINC

CA BUCKEYE HOT SPRINC

OR BUCKHORN CREEK SPRINGS

WY BUFFALO BILL RESERVOIR SPRINGS

NV BUFFALO SPRINGS

NV BUFFALO VALLEY HOT SPRINGS

ID BULL CREEK HOT SPRINCS

OR BULLY CREEK SPRING

CA BUMPASS HELL

ID BURCDORF HOT SPRINCS

NV BURREUL HOT SPRING

NV BUTTE SPRINCS

WY BUTTE SPRINGS

NV BUTTERFIELD SPRINGS

NV BUTTON SPRINGS

CA BYRON HOT SPRINCS

ID CABARTON HOT SPRING

AR CADDO GAP SPRINCS

NV CAINE SPRING

WY CALCITE SPRINGS

AZ CALICHE SPRINC

CALIENTE HOT SPRINCS

CA CALIFORNIA HOT SPRINCS

CA CALISTOGA HOT SPRINCS

MT CAMAS HOT SPRINGS

CA CAMETA WARM SPRING

UT CAMP WILLIAMS

CA CAMPBELL HOT SPRINGS

NV CAMPBELL RANCH SPRINCS

NV CANE SPRINCS

CO CANON CITY HOT SPRINGS

OR CANTERS HOT SPRINC

CA CANYON SPRINC

CAPE HORN WARM SPRINGS

TX CAPOTE SPRINGS

NV CARLIN HOT SPRINGS

NV CARLOTTI RANCH SPRINCS

CA CARLSBAD SPRING

CA CARNEROS SPRINC

NV CARSON HOT SPRINCS

MT CARTER BRIDCE WARM SPRINCS

CA CASA DIABLO HOT POOL
State Spring name

CA CASA DIABLO HOT SPRINGS AND GEYSER

AZ CASA GRANDE SPRINC

WY CASCADE GROUP

CASCADE RESERVOIR HOT SPRING

AZ CASSADORE SPRINC

UT CASTILLA SPRINGS

AZ CASTLE HOT SPRINCS

CA CASTIE ROCK SPRINGS

CO CEBOLLA HOT SPRINGS

NV CEDAR SPRING

CO CEMENT CREEK HOT SPRINC

CA CHANCE SPRINC

CA CHAPPO SPRING

NV CHARNOCK SPRINCS

AK CHENA HOT SPRINCS

NV CHERRY CREEK HOT SPRINGS

MT CHICO HOT SPRINCS

AK CHIEF SHAKES HOT SPRINCS

NV CHILLIS HOT SPRING

NV CHIMNEY WARM SPRING

WY CHOCOLATE POTS

AK CHUGINADAK HOT SPRINCS

AK CIRCLE HOT SPRINCS

WY CISTERN SPRINC

ID CLARENDON HOT SPRINGS

AK CLEAR CREEK HOT SPRINCS

NM CLEAR WATER SPRINC

ID CLEVELAND HOT SPRINCS

AZ CLIFTON HOT SPRINGS

CA COALINGA MINERAL SPRINCS

AZ COFER HOT SPRING

WY COFFEE POT HOT SPRINGS

ID COLGATE WARM SPRINCS

NV COLLAR AND ELBOW SPRING

WA COLLINS HOT SPRINGS

AZ COLORADO POOL

UT COMO SPRINCS

UT COMPTON SPRING

ID CONDIE HOT SPRINCS

CO CONUNDRUM HOT SPRINCS

OR COOK CREEK HOT SPRINC

NM COOK SPRINC

AZ COOLIDGE DAM WARM SPRINC

UT COOPER HOT SPRINGS

ID CORDER HOT SPRING

CA CORN SPRING 
CORWIN HOT SPRINC

CA COSO HOT SPRINGS

CO COTTONWOOD HOT SPRINCS

OR COUGAR RESERVOIR HOT SPRINCS

COUNCIL MTN HOT SPRINGS

COVE CREEK HOT SPRINC

COX HOT SPRINGS

COYOTE HOLE SPRING

COYOTE HOT SPRING

COYOTE SPRING

COYOTE SPRING

CRABTREE HOT SPRINCS

AK CRAIC HOT SPRINCS

CRANE CREEK HOT SPRINGS

OR CRANE HOT SPRINGS

CRATER HOT SPRINC
UT CRATER HOT SPRINGS

WY CRAWFISH CREEK HOT SPRINGS

CRESCENT VALLEY HOT SPRINCS

CA CROHARE SPRING

ID CRONKS CANYON HOT SPRING

OR CRUMP GEYSER

OR CRUMP SPRINC

UT CRYSTAL HOT SPRINCS

NV CRYSTAL POOL

OR CRYSTAL SPRING

OR CRYSTAL SPRING

NV CRYSTAL SPRINGS

UT CUTLER WARM SPRING

ID DAGGER CREEK HOT SPRINC

WY DAISY GROUP

AK DALL HOT SPRINGS

AK DALTON HOT SPRINCS

ID DAN HODGES HOT SPRING

ID DAN HOD CREEK HOT SPRING

ID DANSKIN CREEK HOT SPRINC

NV DARROUGHS HOT SPRINGS

ID DASH CREEK HOT SPRINCS

UT DAVIS

NV DAVIS RANCH SPRING

CA DAVIS SODA SPRING

CA DE IUZ WARM SPRINGS

WY DE MARIS SPRINCS

OR DEER BUTTE HOT SPRINC

OR DEER BUTTE HOT SPRIN
ID DEER HOT SPRINGS

MT DEERLODGE PRISON RANCH SPRINGS

CA DEHY HOT SPRING

\section{State \\ Spring name}

NV DELMUES SPRINCS

CA DELONECHA HOT SPRINGS

CA DEMOCRAT HOT SPRINGS

AK DENIKTOW RIDGE HOT SPRINCS

NM DERRY WARM SPRINGS

UT DESERET LIVESTOCK

UT DESERET LIVESTOCK SOUTH SPRINC

UT DESERET LIVESTOCK SOUTH SPRINCS

NV DEVILS HOLE

CA DEVILS KITCHEN

CO DEXTER SPRING

UT DIAMOND FORK WARM SPRINGS

NV DIANAS PUNCH BOWL

NV DIMONTE SPRINC

CA DIRTY SOCKS HOT SPRING

NV DIXIE COMSTOCK MINE

NV DIXIE HOT SPRINCS

UT DIXIE HOT SPRINGS

CA DOLANS HOT SPRINC

ID DOLLAR CREEK WARM SPRING

ID DONLAY RANCH HOT SPRING

WA DORR FUMAROLE FIELD

CA DOS PALMAS SPRING

CO DOTSERO WARM SPRINCS

UT DOTSONS WARM SPRING

NV DOUBIE DIAMOND SPRING

NV DOUBLE HOT SPRINCS

OR DOUBLE O BARNYARD SPRINC

OR DOUBLE O SPRINC

NV DOUBLE SPRINC

NV DOUD SPRINGS

WY DOUGLAS HOT SPRINCS

ID DOWNATA HOT SPRINCS

CA DRAKESBAD

OR DUNN SPRINC

CO DUNTON HOT SPRING

MT DURFEE CREEK SPRINGS

ID DURFEE SPRING

ID DUTCH FRANK HOT SPRING

NV DYKE HOT SPRINCS

AZ EACLE CREEK HOT SPRINCS

OR EAGLE POINT SPRINC

NV EAGLE SALT WORKS SPRINC

ID EASLEY HOT SPRINGS

UT EAST IORDAN CANAL

OR EAST LAKE HOT SPRINCS
State Spring name

WY EBRO SPRINCS

ECHINUS GEYSER

EDEN HOT SPRINGS

EL ENCINO SPRINCS

EL. OJO ESCONDIDO

ELDORADO SPRINGS

CA ELGIN MINE

ELK CREEK HOT SPRING

TIKHORN HOT SPRINCS

EIKHORN WARM SPRING

NV ELKO HOT SPRING

ELISON RANCH WARM SPRINC

CA ELSINORE HOT SPRINGS

WY EMERALD POOL

NV EMIGRANT SPRINCS

CA ENCLAND SPRINGS

MT ENNIS HOT SPRINGS

CA ESSEX SPRINGS

WY EXCELSIOR GEYSER CRATER

NV FAIRBANKS SPRING

ID FAIRCHILD HOT SPRING

CA FAIRVIEW HOT SPRING

WY FAIRY SPRINCS

CA FALES HOT SPRINCS

ID FALL CREEK MINERAL SPRINCS

VA FALLING SPRING

UT FAULT ZONE SPRING

NM FAYWOOD HOT SPRINCS

NV FERNLEY HOT SPRINGS

CA FISH CREEK HOT SPRINCS

WA FISH HATCHERY WARM SPRING

CA FISH SPRINCS

NV FISH SPRINCS

UT FISH SPRINCS

OR FISHER HOT SPRINGS

WY FIVE SISTERS SPRINCS

NV FIVEMILE SPRING

OR FIVEMILE SPRING

NV FLAG SPRINGS

OR FLACSTAFF BUTTE HOT SPRING

WY FLAT CONE SPRING

NV FLATNOSE RANCH SPRINC

WY FLOOD GROUP

NV FLY RANCH HOT SPRINGS

NV FLYNN RANCH SPRINCS 


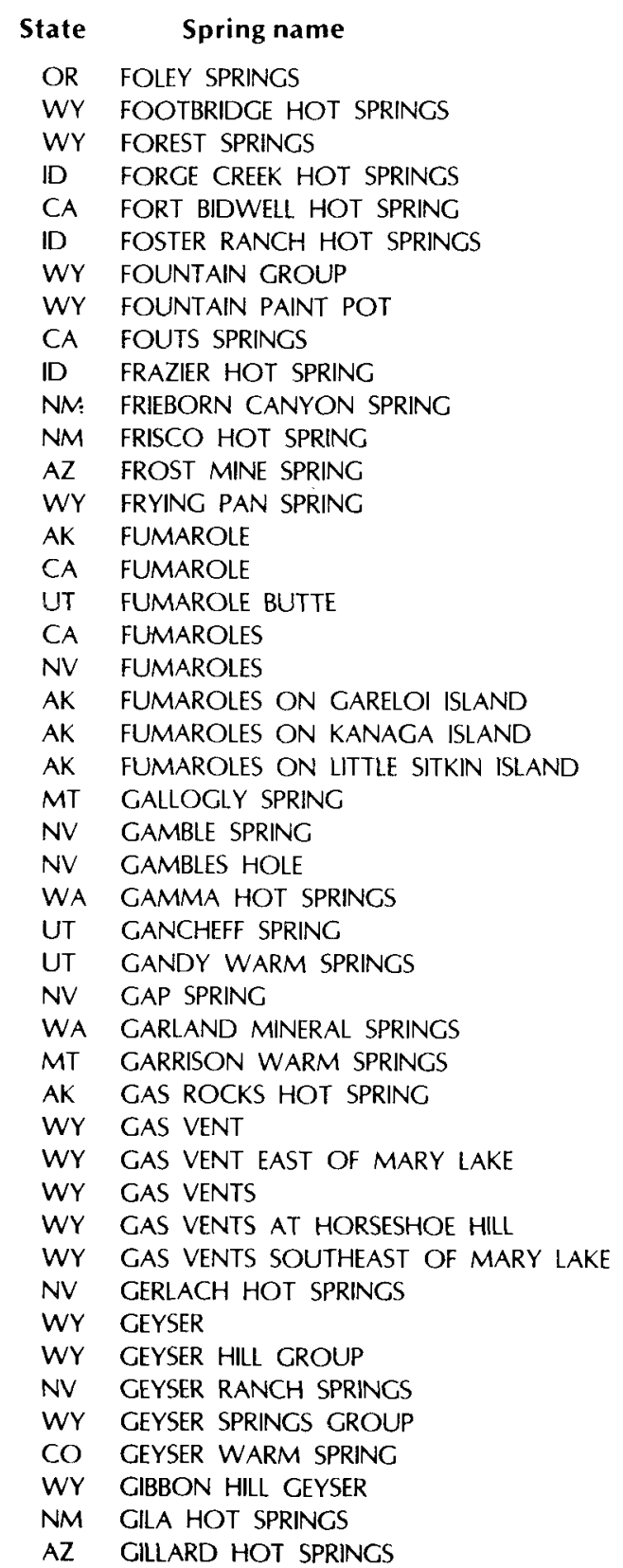

\begin{tabular}{ll} 
State & \multicolumn{1}{c}{ SPRIII name } \\
CA & GILMAN HOT SPRINGS \\
CA & GILROY HOT SPRINGS \\
NV & GIOCOECHA WARM SPRINGS \\
ID & GIVENS HOT SPRINGS \\
CA & GLEN IVY HOT SPRINCS \\
CO & GLENWOOD SPRINGS \\
NM & GOAT CAMP SPRING \\
ID & GOAT HOT SPRINCS \\
AK & GODDARD HOT SPRINCS \\
NV & COLCONDA HOT SPRING \\
ID & GOLD FORK HOT SPRING \\
ID & GOLDBUG HOT SPRING \\
WA & GOIDMEYER HOT SPRINCS \\
OR & GOODMAN SPRING \\
CA & GORDON WARM SPRINGS \\
UT & GOSHEN WARM SRINCS \\
ID & GRANDIEAN HOT SPRING \\
ID & GRANITE CREEK HOT SPRING \\
WY & GRANITE FALLS HOT SPRINGS \\
WY & GRANITE HOT SPRING \\
AK & GRANITE MOUNTAIN HOT SPRINCS \\
MT & GRANITE SPRINCS \\
UT & GRANTSVILL WARM SPRINGS \\
ID & GRAPE CREEK WARM SPRING \\
AZ & GRAPEVINE SPRING \\
CA & GRAPEVINE SPRINGS \\
NV & GREAT BOIING SPRING \\
ID & GREEN CANYON HOT SPRINGS \\
WA & GREEN RIVER SODA SPRING \\
UT & GREEN SPRING \\
MT & GREEN SPRINGS \\
MT & GRECSON HOT SPRINGS \\
CA & GRIZZIY SPRING \\
ID & GROUND HOG HOT SPRINGS \\
CA & GROVERS HOT SPRINGS \\
CA & GROWLER HOT SPRING \\
ID & GUYER HOT SPRINGS \\
ID & HAILEY HOT SPRINGS \\
OR & HALLINAN SPRING \\
NV & HAMMOND RANCH SPRING \\
AZ & HANNAH HOT SPRING \\
CA & HARBIN SPRINCS \\
OR & HARDBOARD SPRING \\
CO & HARTSEL HOT SPRINGS \\
UT & HATTON HOT SPRING \\
ID & HAVEN LODGE HOT SPRING \\
\end{tabular}

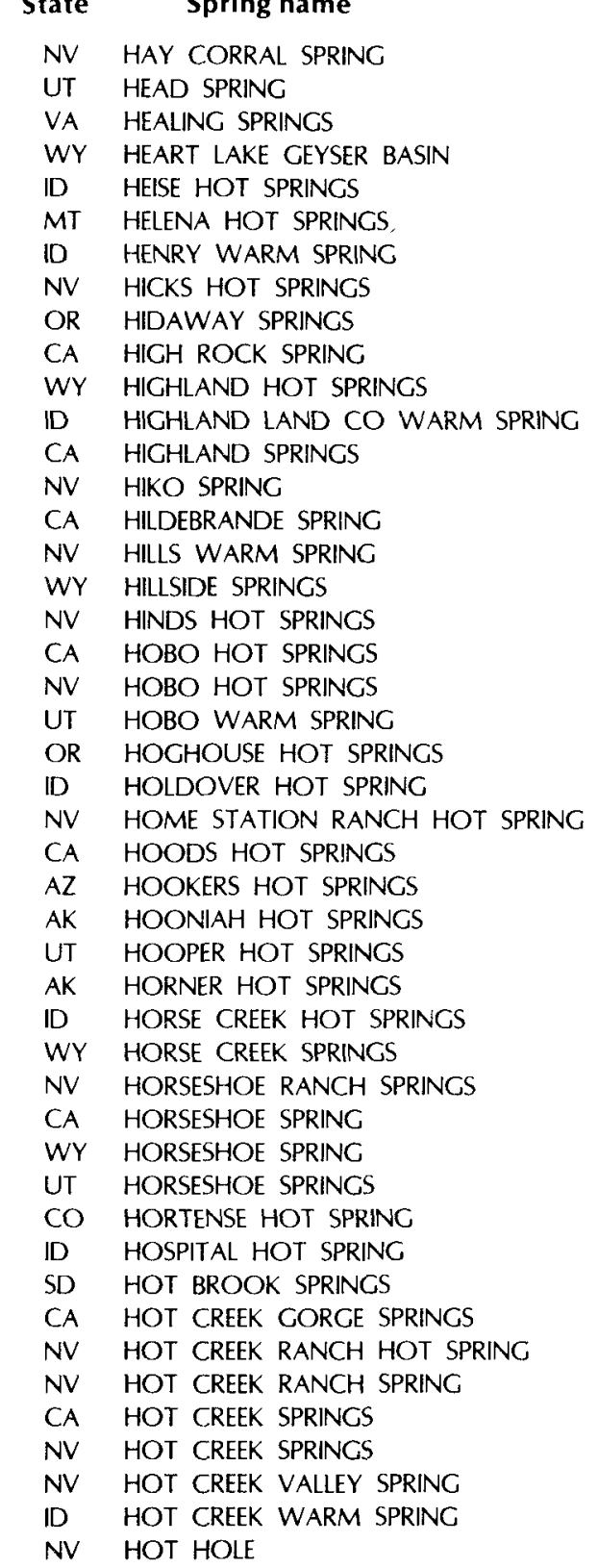


OR HOT LAKE

WA HOT LAKE

AK HOT LAKE IN BOTTOM OF CRATER PEAK

OR HOT LAKE SPRINGS

NV HOT POT

WY HOT RIVER

NV HOT SEEPS (SAN EMIDIO DESERT)

CA HOT SPOT

AZ HOT SPRINC

AZ HOT SPRING

CA HOT SPRING

OR HOT SPRING

WY HOT SPRINC

AK HOT SPRING AT WEST END EMMONS LAKE

WY HOT SPRING BASIN GROUP

WY HOT SPRING BASIN GROUP

AK HOT SPRING NEAR ARRICETCH PEAKS

AK HOT SPRING NEAR FISH BAY

AK HOT SPRING NEAR FLAT

AK HOT SPRING NEAR KATMAI PASS

AK HOT SPRING NEAR KWINIUK RIVER

AK HOT SPRING NEAR LITTLE MINOOK CR

AK HOT SPRING NEAR MOTHER GOOSE LAKE

AK HOT SPRING NEAR PORT MOLLER

AK HOT SPRING NEAR TULUKSAK RIVER

AK HOT SPRING NORTH ARM PERIL STRAIT

AK HOT SPRING ON AMACAT ISLAND

AK HOT SPRING ON ATTU ISLAND

AK HOT SPRING ON FLAT CREEK

AK HOT SPRING ON KAGAMIL ISLAND

AK HOT SPRING ON UITTLE SITKIN ISLAND

WY HOT SPRING ON MOSS CREEK

AK HOT SPRING ON SEGUAM ISLAND

AK HOT SPRING ON TANAGA ISLAND

AK HOT SPRINC ON TRIBUTARY OF INNOKO R

AK HOT SPRING ON TWELVEMILE CREEK

AK HOT SPRING ON UMNAK ISLAND

AK HOT SPRING ON UNIMAK ISLAND

AK HOT SPRING WEST OF CAPE DOUGLAS

AR HOT SPRINGS

CA HOT SPRINGS

$\begin{array}{ll}\text { CA } & \text { HOT SPRINCS } \\ \text { ID } & \text { HOT SPRINGS }\end{array}$

NC HOT SPRINGS

NM HOT SPRINGS

NV HOT SPRINCS

SD HOT SPRINCS

TX HOT SPRINCS

VA HOT SPRINCS

WY HOT SPRINCS

WY HOT SPRINCS AT SEVENMILE HOLE

WY HOT SPRINGS AT SULPHUR HILLS

AK HOT SPRINCS EAST OF COLD BAY

AK HOT SPRINGS EAST OF DIAMOND

WY HOT SPRINCS EAST OF MARY LAKE

WY HOT SPRINCS IN PELICAN VALLEY

AK HOT SPRINGS NEAR DIVISION BM

AK HOT SPRINCS NEAR DULBI RIVER

AK HOT SPRINCS NEAR GEYSER BICHT

AK HOT SPRINGS NEAR GLACIER

AK HOT SPRINCS NEAR HOT SPRINCS BAY

AK HOT SPRINCS NEAR HOT SPRINCS COVE

AK HOT SPRINGS NEAR ICY POINT

AK HOT SPRINGS NEAR LAVA CREEK

AK HOT SPRINGS NEAR MAKUSHIN VOLCANO

AK HOT SPRINGS NEAR MORZHOVOI

AK HOT SPRINCS NEAR MT KATMA

AK HOT SPRINCS NEAR OPHIR CREEK

AK HOT SPRINGS NEAR SOUTH BM

AK HOT SPRINCS NEAR SUMMER BAY

AK HOT SPRINCS NORTH END TENAKEE INIET

AK HOT SPRINCS ON ADAK ISLAND

AK HOT SPRINGS ON AKUN ISLAND

AK HOT SPRINGS ON ATKA ISLAND

AK HOT SPRINCS ON BIG WINDY CREEK

WY HOT SPRINGS ON BOC CREEK

AK HOT SPRINGS ON BOGOSLOF ISLAND

WY HOT SPRINGS ON CONTINENTAL DIVIDE

AK HOT SPRINCS ON GREAT SITKIN ISLAND

AK HOT SPRINGS ON HAWK RIVER

WY HOT SPRINCS ON LEWIS LAKE

AK HOT SPRINGS ON LOWER RAY RIVER

AK HOT SPRINGS ON UPPER CHUILNUK RIVER

WY HOT SPRINGS ON UPPER FIREHOLE RIVER

WY HOT SPRINGS ON UPPER SNAKE RIVER

WY HOT SPRINGS ON UPPER SOUR CREEK

NV HOT SPRINGS RANCH

NV HOT SPRINGS RANCH SPRINCS

CA HOT SPRINCS VALLEY

ID HOT SULPHUR LAKE

\section{State Spring name}

CO HOT SUIPHUR SPRINGS

ID HOT SUIPHUR SPRINGS

NV HOT SULPHUR SPRINCS

WY HOT, ON LAMAR RIVER

OR HOWARD HOLIDAY

NV HOWARD HOT SPRING

CA HOWARD SPRINGS

WY HUCKLEBERRY HOT SPRINGS

NV HUFFAKER SPRINGS

OR HUGHET SPRINC

CA HUNT HOT SPRING

MT HUNTERS HOT SPRINCS

OR HUNTERS HOT SPRINGS

AK HUTLINANA HOT SPRINGS

NV HYDER HOT SPRINCS

CO IDAHO SPRINGS

WY IMMICRANTS WASHTUB

WY IMPERIAL GEYSER

INDIAN BATHTUB HOT SPRINCS

NM INDIAN BIG SPRINC

ID INDIAN CREEK HOT SPRING

AZ INDIAN HOT SPRINGS

ID INDIAN HOT SPRINGS

TX INDIAN HOT SPRINGS

ID INDIAN SPRINCS

NM INDIAN SPRINGS

NV INDIAN SPRINC:

CA INDIAN VALLEY HOT SPRINCS

WY INKWELL

CA IRIDAT SPRINC

WY IRON SPRING

UT IRVINE SPRING

HI ISAAC HALE PARK SPRING

NV IZZENHOOD RANCH SPRINCS

NV IACK RABBIT SPRING

OR JACKSON HOT SPRINGS

$W Y$ IACKSON LAKE HOT SPRINCS

CA IACKSON VALLEY MUD SPRINCS

CA IACUMBA HOT SPRINCS

WY IAKEYS FORK SPRING

OR IAMIESON HOT SPRINCS

MT IARDIN!E HOT SPRINC

NM IEMEZ SPRINCS

ID JERRY JOHNSON HOT SPRINGS

OR JOAQUIN MILLER HOT SPRINGS

NV IOHNSON RANCH SPRINGS 


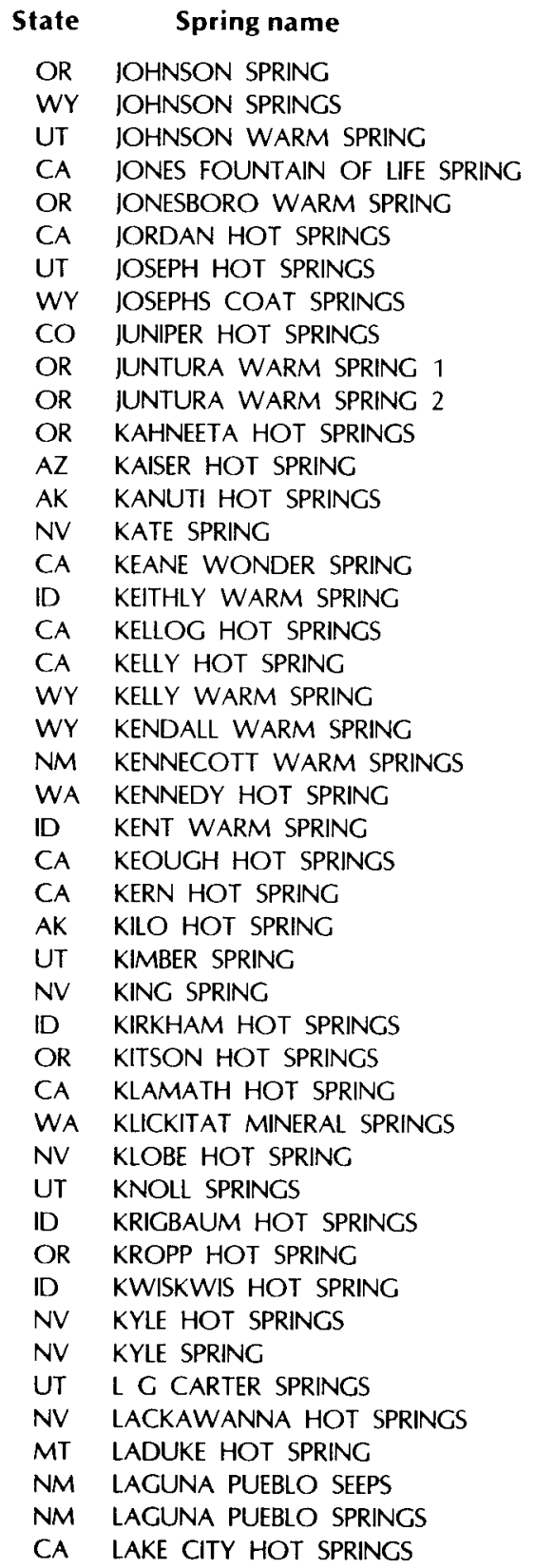

\section{State}

LAKE WALCOTT HOT SPRING

CA LAKEVIEW HOT SPRINCS

DAKEY HOT SPRING

MT LANDUSKY PLUNCE

MT LANDUSKY SPRINGS

UT LARSON SPRINGS

CA LAS CRUCES HOT SPRINCS

NV LAS VEGAS SPRINGS

ID LATTY HOT SPRINC

ID LAVA HOT SPRINCS

AZ LAVA WARM SPRINCS

UT LAVERKIN HOT SPRINCS

NT LAVERIN HOT SPRINCS

VA LAWTON HOT SPRINCS

$V A$ LAYTON SPRING

NV LEACH HOT SPRINGS

NY LEBANON SPRINC

NV LEE HOT SPRINCS

UT LEFEVRE

ID LEGGIT CREEK HOT SPRINC

OR LEHMAN SPRINGS

OR LEITHEAD HOT SPRING

CO LEMON HOT SPRINC

CA LEONARDS HOT SPRINCS

WA LESTER HOT SPRINCS

AZ LEWIS SPRINC

ID LICK CREEK WARM SPRINC

ID LIDY HOT SPRINGS

GA LIFSEY SPRINC

ID LUGHTFOOT HOT SPRINCS

OR LIMEKILN HOT SPRING

UT LINCOLN POINT WARM SPRINGS

IO LITTLE ANTELOPE FLAT WARM SPRINC

CA LITTLE CALIENTE SPRINC

CA LITTLE GEYSERS

CA LITTLE HOT CREEK SPRINC

CA UTTLE HOT SPRING

NV LITTLE HOT SPRINGS

CA LITTLE HUNTER CANYON SPRINCS

AK LITTLE MELOZITNA HOT SPRINGS

UT LITTLE MOUNTAIN HOT SPRINC

FL LITTLE SALT SPRING

WY LITTLE SHEEP MOUNTAIN SPRINC

NV LITTLE WARM SPRINC

WY LITTLE WARM SPRING

MT LITTLE WARM SPRINGS

UT LIVINGSTON WARM SPRINCS

\section{State Spring name}

NV LOCKES BIG SPRINC

MT LODGEPOLE SPRINGS

ID LOFTUS HOT SPRINC

MT IOLO HOT SPRINGS

WY LONE STAR GEYSER

WY LONE STAR GEYSER BASIN

CA LONE TREE MINERAL SPRINC

WA LONGMIRE MINERAL SPRINCS

NV LONCSTREET SPRING

CA LOS GUILICOS WARM SPRINCS

OR LOST CABIN SPRINC

MT LOVELLS HOT SPRING

ID LOWER BIRCH SPRING

ID LOWER BOWERY HOT SPRINC

ID LOWER INDIAN BATHTUB HOT SPRINGS

AK LOWER KLAWASI CONE

ID LOWER LOON CREEK HOT SPRINC

NV LOWER RANCH HOT SPRINCS

NV LOWER SHELLBOURNE SPRINC

CA LOWER WARM SPRINCS

CO LOWER WAUNITA HOT SPRINCS

OR LUCE HOT SPRINGS

NM LYONS HUNTING LODGE HOT SPRINGS

NV MACFARLANE HOT SPRING

UT MADSENS HOT SPRINGS

ID MAGIC HOT SPRINCS

AK MAKUSHIN VOLCANO FUMAROIES

ID MALAD WARM SPRINC

WY MAMMOTH HOT SPRINGS

NM MANBY HOT SPRINGS

NM MANGAS SPRINCS

AK MANLEY HOT SPRINCS

NV MANSE RANCH SPRING

CA MANZANITA MINE SPRINCS

ID MAPLE GROVE HOT SPRINCS

CA MARK WEST SPRINCS

ID MARSH CREEK HOT SPRING

ID MARSH GULLY HOT SPRINCS

ID MARTEN HOT SPRINGS

CA MATILIA HOT SPRINCS

NM MCCAULEY HOT SPRINGS

CA MCCOY SPRINC

NV MCCOY SPRINCS

OR MCCREDIE SPRINCS

NV MCGILL SPRING

CA MCLEAR WARM SPRINCS 
State

\section{Spring name}

MCLEOD RANCH HOT SPRINC

MT MCMENOMEY RANCH SPRINC

UT MEADOW HOT SPRINCS

OR MEDICAL HOT SPRINCS

MT MEDICINE HOT SPRINCS

MT MEDICINE SPRINCS

AK MELOZI SPRINCS

AK MELOZITNA SPRINGS

MENLO BATHS

MERCER SPRING

MERCEY HOT SPRINGS

MESCAL WARM SPRING

MICKEY SPRINCS

MIDDLE FORK INDIAN CREEK HOT SPRING

WY MIDDLE GROUP

WY MIDDLE GROUP

MIDWAY HOT SPRING

UT MIDWAY HOT SPRINCS

WY MIDWAY PICNIC GROUND

D MILFORD SWEAT HOT SPRING

CA MILL CREEK SPRINCS

OR MILLPOND HOT SPRING

NM MIMBRES HOT SPRINGS

NV MINERAL HOT SPRING

CO MINERAL HOT SPRINCS

AK MINERAL SPRINC

CA MINERAL SPRINGS

WV MINNEHAHA SPRINCS

CA MIRACLE HOT SPRINCS

MIRACIE HOT SPRINCS

MITCHELL BUTTE HOT SPRINC

MITCHEIL SPRINC

MIZE SPRINC

MOANA SPRINGS

MOAPA WARM SPRINCS

WA MOFFETTS HOT SPRINGS

MOLLYS HOT SPRNC

MONKEY SPRINC

MONO BASIN WARM SPRINGS

MONO HOT SPRINCS

MONROE HOT SPRINCS

MONTE NEVA HOT SPRINCS

MONTECITO HOT SPRINGS

MONTEZUMA HOT SPRINCS

MONUMENT GEYSER BASN

MVON RIVER SPRINC
State

\section{Spring name}

NV MOORMAN SPRING

ID MORES CREEK HOT SPRINC

CA MORGAN HOT SPRING

UT MORGANS WARM SPRING

ID MORMON BEND HOT SPRINC

ID MORMON RANCH WARM SPRING

ID MORMON RESERVOIR WARM SPRINC

WY MORNING GLORY POOL

WY MORNING MIST SPRINGS

NM MOSLEY SPRINC

OR MOSS RANCH SPRINC

OR MOSS RANCH SPRINCS

NV MOUND SPRINCS

ID MOUND VALLEY WARM SPRINC

WA MOUNT ADAMS FUMAROLES

OR MOUNT HOOD FUMAROLES

CO MOUNT PRINCETON HOT SPRINGS

OR MOUNT VERNON HOT SPRINGS

WA MT BAKER FUMAROLES

WA MT RAINIER FUMAROLES

WA MT ST HELENS FUMAROLES

WY MUD GEYSERS

CA MUD POTS

NV MUD SPRINCS

WY MUD VOLCANO

CA MUD VOLCANOES

NV MUDDY SPRINC

CA MUIR SPRINCS

ID MURPHY HOT SPRINCS

CA MURRIETTA HOT SPRINCS

NV MUSTANG SPRINGS

CA MYERS WARM SPRING

CA MYERS WARM SPRI

WY MYRIAD GROUP
$W Y$ MYSTIC FALLS SPRINGS

WY MYSTIC FALLS

CA NAPA ROCK SODA SPRING

CA NAPA VICHY SPRINCS

ID NAT-SOO-PAH WARM SPRINC

OR NEAL HOT SPRINGS

WY NEEDLE HOT SPRINCS

OR NEEDLE HOT SPRINCS

NV NEVADA HOT SPRINGS

NV NEVADA STATE PRISON SPRING

CA NEVARES SPRINGS

MT NEW BILTMORE HOT SPRINCS

VA NEW RIVER WHITE SULPHUR SPRINGS

\section{Spring name}

CA NEWBERRY SPRINC

CA NEWMAN SPRINGS

CA NEWSOM SPRINCS

NV NILE SPRING

MT NIMROD SPRINCS

NINEMEYER HOT SPRINGS

NV NINEMILE SPRINCS

TX NIXON SPRING

MT NORRIS HOT SPRINCS

WY NORTH BUFFALO FORK SPRINCS

UT NORTH SPRINCS

AK NYLEN HOT SPRINGS

OAKLEY HOT SPRING

AZ OATMAN WARM SPRINGS

UT OGDEN HOT SPRING

WA OHANAPECOSH HOT SPRINCS

NM OIITOS SPRINCS

NM OJO CALIENTE

NM OJO DE LAS CANAS

AK OKPILAK SPRINCS

NV OLD DUCAN RANCH HOT SPRINCS

WY OLD FAITHFUL GEYSER

ID OLD HOMESTEAD WARM SPRINC

WV OLD SWEET SPRING

OR OLENE GAP HOT SPRINGS

WA OLYMPIC HOT SPRINGS

CA ONE SHOT MINING CO

OR OREGON HOT SPRINCS

WA ORR CREEK WARM SPRINCS

CA ORRS SPRINCS

CO ORVIS HOT SPRINC

CO OURAY HOT SPRINGS

OWEN CABIN HOT SPRINC

OWL CREEK HOT SPRINGS

WA PACKWOOD HOT SPRINC

CO PACOSA SPRINCS

NV PAHRUMP SPRING

NV PAINTED HILLS MINE

AZ PAKOON SPRINCS

A PALM SPRING

CA PALM SPRINCS

CA PAN HOT SPRINCS

NV PANACA SPRINC

CA PAOHA ISLAND SPRINGS

ID PARADISE HOT SPRINCS

CA PARADISE SPRINC 


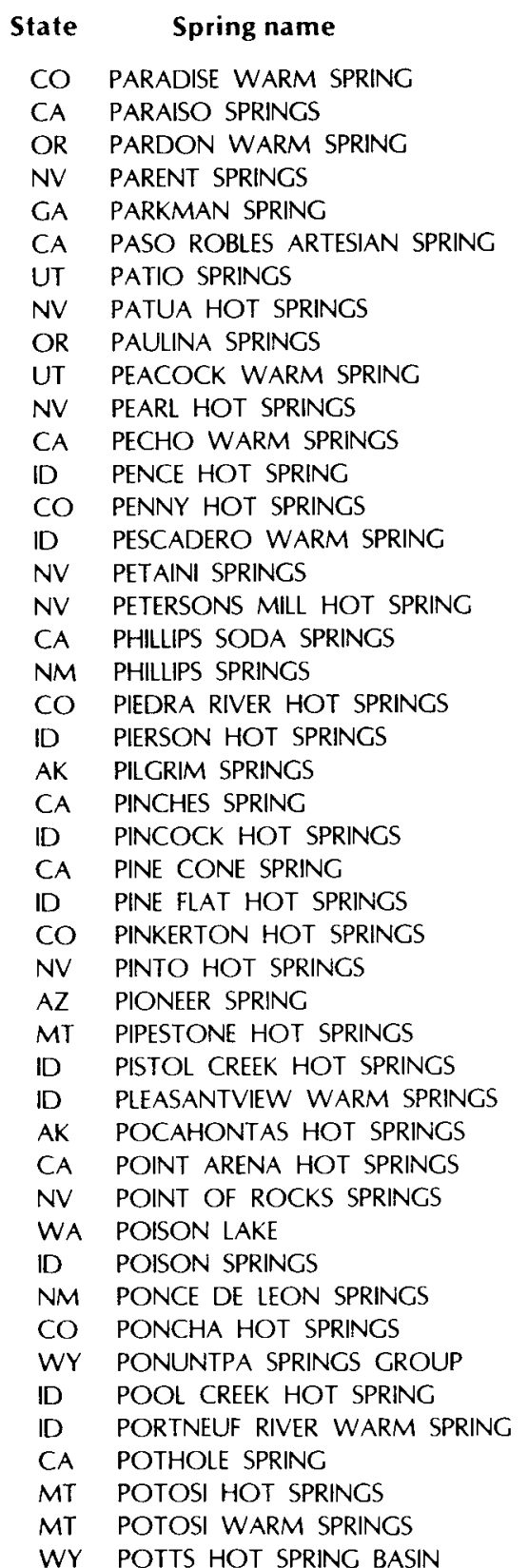

\begin{tabular}{ll} 
State & \multicolumn{1}{c}{ SPRING name } \\
NV & POTTS RANCH HOT SPRINCS \\
UT & POULSEN SPRING (SALT) \\
ID & PREIS HOT SPRING \\
NV & PRESTON SPRINCS \\
ID & PRICES WARM SPRING \\
ID & PROSPECTOR HOT SPRINGS \\
CA & PSEUDO COMPLEXION SPRING \\
UT & PUGSLEY \\
MT & PULLER HOT SPRINGS \\
NV & PYRAMID ISLAND HOT SPRING \\
WY & QUAGMIRE GROUP \\
WY & QUEENS LAUNDRY \\
ID & QUEENS RIVER HOT SPRING \\
CA & QUIGLEY SODA SPRING \\
MT & QUINNS HOT SPRINGS \\
AZ & QUITOBAQUITO SPRINCS \\
WY & RABBIT CREEK GROUP \\
WY & RABBIT CREEK GROUP \\
AZ & RADIUM HOT SPRINCS \\
OR & RADIUM HOT SPRINCS \\
NM & RADIUM SPRINCS \\
UT & RADIUM WARM SPRING \\
CO & RAINBOW HOT SPRINGS \\
NM & RAINBOW SPRING \\
WY & RAINBOW SPRINGS \\
NV & RAINE RANCH SPRINGS \\
NV & RALPHS WARM SPRINGS \\
CO & RANGER HOT SPRING \\
ID & RATTLESNAKE HOT SPRING \\
UT & RAY LUNT SPRING \\
AK & RAY RIVER HOT SPRING \\
TX & RED BULL SPRING \\
UT & RED CANYON \\
UT & RED HILL HOT SPRING \\
AK & RED HILL SPRING \\
ID & RED RIVER HOT SPRINGS \\
UT & REDIUM SPRING \\
CA & REDS MEADOW HOT SPRINGS \\
AK & REED RIVER HOT SPRING \\
MT & RENOVA HOT SPRINGS \\
CA & RESTING SPRING \\
NV & REVEILLE MILL SPRINC \\
CO & RHODES WARM SPRING \\
ID & RICE SPRING \\
UT & RICHFIEID WARM SPRINGS \\
ID & RIGGINS HOT SPRINGS \\
&
\end{tabular}

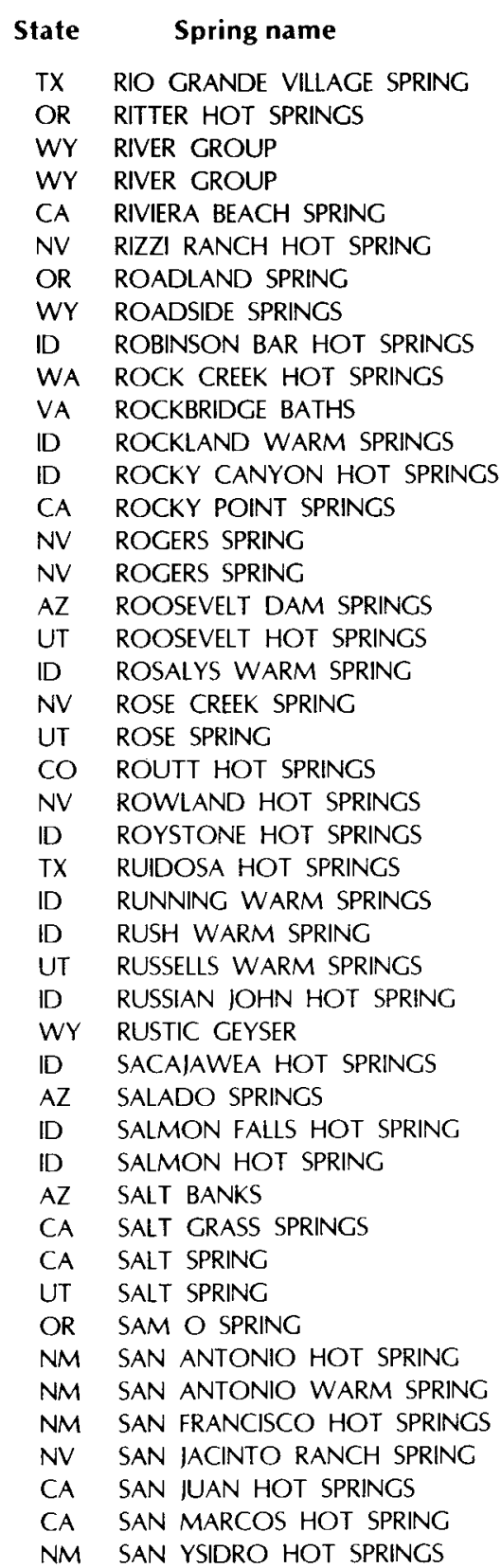


NM SAN YSIDRO WARM SPRINCS

MA SAND SPRINC

NV SAND SPRING

NV SAND SPRINGS

CA SANTA YSABEL SPRINCS

WY SAPPHIRE POOL

NV SARATOGA HOT SPRINC

UT SARATOCA HOT SPRINGS

WY SARATOCA HOT SPRNCS

CA SARATOGA SPRING

CA SARGENT ESTATE WARM SPRING

NV SAULSBURY WARM SPRING

WA SCENIC HOT SPRINGS

NV SCHOOLHOUSE SPRING

OR SCOTT SPRING

CA SCOVERN HOT SPRINGS

NV SCRUGGS SPRINGS

ID SECESH HOT SPRING

WY SECRET VALLEY HOT SPRINCS

NM SEDILLO SPRINC

AZ SEEP

NV SEEP

CA SEIGLER SPRINGS

CA SEIGLER SPRINCS

WY SEMI-CENTENNIAL GEYSER

NV SENATOR FUMAROLES

WY SENTINEL CEYSERS

AK SERPENTINE HOT SPRINCS

CA SESPE HOT SPRINCS

NV SEVEN DEVILS HOT SPRINCS

WY SEVENMILE HOLE

CA SEYFERTH HOT SPRINGS

ID SHARKEY HOT SPRING

CO SHAW SPRINGS

ID SHEEP CREEK BRIDGE HOT SPRING

ID SHEEP CREEK HOT SPRINC

CA SHEEP CREEK SPRINC

WY SHEEP MOUNTAIN SPRINGS

ID SHEEPEATER HOT SPRINCS

WY SHELF SPRING

WA SHERMAN CRATER FUMAROLES

NV SHIPLEY HOT SPRINC

WY SHOSHONE CEYSER BASIN

WY SHOSHONE HOT SPRINCS

\section{State Spring name}

CA SHOSHONE SPRING

SHOWER BATH SPRINGS

SILVER CREEK HOT SPRINC

MT SILVER STAR HOT SPRINGS

NV SILVERPEAK HOT SPRINC

WA SIMCOE SODA SPRINGS

NV SIRI RANCH SPRING

OR SITZ WARM SPRING

CA SKACCS SPRINGS

SKILLERN HOT SPRINGS

SLATE CREEK HOT SPRINC

CA SLATES HOT SPRINCS

MT SLEEPING CHILD HOT SPRINGS

MT SLOAN COW CAMP SPRINC

ID SMITH CABIN HOT SPRIN

NV SMITH RANCH SPRINCS

WY SMOKE IUMPER HOT SPRINCS

WY SNAKE HOT SPRINGS

OR SNIVELY HOT SPRINC

UT SNOW SPRINC

ID SNOWSHOE JOHNSONS HOT SPRING

CA SOBODA HOT SPRINCS

$N M$ SOCORRO SPRINC

NM SODA DAM HOT SPRINGS

NV SODA LAKE

AZ SODA SPRING

CA SODA SPRINC

NV SODA SPRING

CA SODA SPRING IN CACHE FORMATION

CA SODA SPRINGS

ID SODA SPRINCS

NV SODA SPRINGS

CA SODA STATION SPRINGS

WA SOL DUC HOT SPRINGS

WY SOLITARY GEYSER

NV SOU HOT SPRINGS

NM SOUSE SPRINCS

OR SOUTH BLACK WILLOW SPRING

CO SOUTH CANYON HOT SPRINGS

WY SOUTH ENTRANCE HOT SPRINGS

ID SOUTH FORK PLUNGE

NV SOUTH MOSQUITO CREEK RANCH SPRINGS

AK SOUTH STIKINE SPRINC

UT SOUTH TULE SPRING

WY SOUTHEASTERN GROUP

NM SPENCE HOT SPRINGS

\section{State Spring name}

NV SPENCER HOT SPRINCS

CA SPIERS SPRINCS

UT SPLIT MOUNTAIN WARM SPRINGS

WY SPRAY GEYSER

AZ SPRING

CA SPRING

NM SPRING

NV SPRING

AZ SPRINC (HOT)

CA SPRING (HOT)

NM SPRING (HOT)

NV SPRING (HOT)

OR SPRING (HOT)

UT SPRING (HOT)

NM SPRING (SALT)

CA SPRING IN ONELLL FOREBAY

NM SPRING MFG FOUR

NM SPRING MFG ONE

NM SPRING MFC THREE

NM SPRING MFC TWO

AR SPRING ON LITTLE MISSOURI RIVER

AR SPRING ON REDIAND MOUNTAIN

AZ SPRINGS

CA SPRINGS

NV SPRINGS

OR SPRINCS

UT SPRINCS

NV SPRINCS (HOT)

OR SPRINCS (HOT)

TX SPRINCS (LAS CIENECAS)

CA SPRINCS AT TECOLOTE TUNNEL

WY SPRINCS ON FAIRY CREEK

CA SQUAW BATHS

D SQUAW HOT SPRINGS

CA ST HELENA WHITE SULPHUR SPRING

WA ST MARTINS HOT SPRINGS

D STANLEY HOT SPRING

ID STANEEY HOT SPRINGS

ID STARKEY HOT SPRINCS

MT STATE HOSPITAL WARM SPRINGS

NM STATUE SPRINC

MT STAUDENMEYER SPRINGS

HI STEAM VENTS

WY STEAM VENTS AT ROARING MOUNTAIN

D STEAMBOAT HOT SPRING

CO STEAMBOAT SPRINGS 


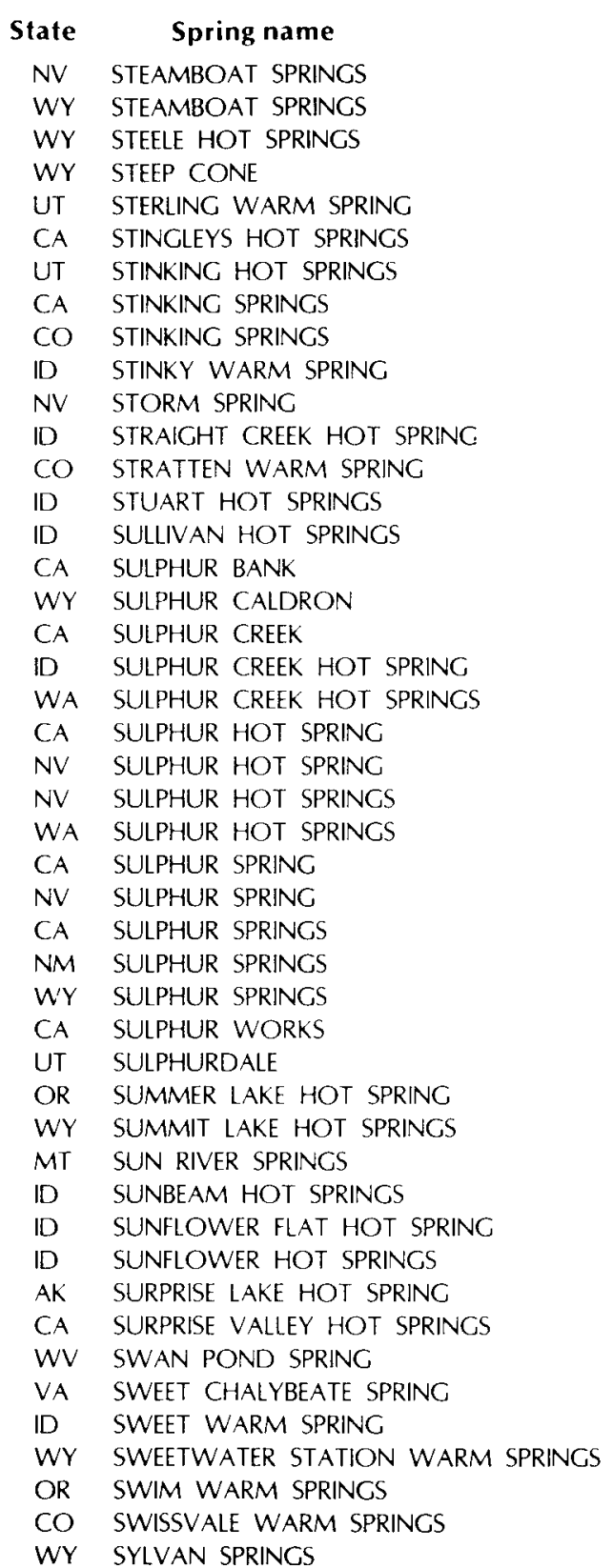

\author{
State Spring name \\ CA TABLE MOUNTAIN (SPRING) \\ D TADPOLE WARM SPRING \\ CA TASSAIARA HOT SPRINCS \\ GA TAYLOR SPRINC \\ ID TAYLOR SPRINCS \\ UT TEBBS \\ CA TECOPA HOT SPRINCS \\ CA TECOPA HOT \\ CA TEMECULA HOT SPRINCS \\ AK TENAKEE HOT SPRINCS \\ CA TERMINAL GEYSER \\ ID TERTELING HOT SPRINGS \\ CA THE GEYSERS \\ NV THE GEYSERS \\ CA THE HOT SPRINCS \\ NV THE HOT SPRINGS \\ NM THE MEADOWS WARM SPRING \\ WY THE MUDKETTLES \\ WY THE MUDKETTLES
WY THE MUSHPOTS \\ NV THE NEEDLE ROCKS HOT SPRING \\ MT THE PLUNCE \\ CA THE TUB \\ AK THERMAL SPRINCS IN OKMOK CAIDERA \\ UT THERMO HOT SPRINCS \\ WY THERMOPOLIS HOT SPRINCS \\ $\begin{array}{ll}W Y & \text { THERMOPOLIS HOT SPRINGS } \\ \text { ID THOMAS CREEK RANCH HOT SPRINO }\end{array}$ \\ ID THOMAS FLATS WARM SPRING \\ NV THOMPSON RANCH SPRINC \\ WV THORN SPRING \\ ID THOROUCHBRED WARM SPRINCS \\ NV THREEMILE SPRING \\ GA THUNDERING SPRINC \\ CA TIPTON SPRINGS \\ CA TOLENAS SPRINGS \\ AK TOLOVANA HOT SPRINGS \\ GA TOM BROWN SPRING \\ AZ TOM BROWN WARM SPRING \\ NV TOM SPRINC \\ AZ TONTO NATURAL BRIDCE SPRINC \\ CA TOPHET HOT SPRINCS \\ WY TORTOISE SHELL \\ D TOWNE CREEK WARM SPRINC \\ ID TRAIL CREEK HOT SPRING \\ CA TRAVERTINE HOT SPRINCS \\ CA TRAVERTINE SPRINGS \\ ID TREASURETON HOT SPRINGS
}

\section{Spring name}

NV TREGO HOT SPRINGS TRIMBLE HOT SPRINC TRIPP HOT SPRINCS TROUT CREEK SPRINC TRUDAU WARM SPRINGS TRUTH OR CONSEQUENCES TSCHANNE HOT SPRINC

TUDORS SPRING

TULE SPRINC

TUNALKIEN LAKE HOT SPRINC

WY TURBID SPRINCS

NM TURKEY CREEK HOT SPRING

CA TUSCAN SPRINGS

TWELVEMILE SPRING

TWIN LAKES HOT SPRINCS

TWIN SPRING

TWIN SPRINCS

TWIN SPRINGS

TYLERS BATH SPRINC

UDY HOT SPRINCS

UMPQUA HOT SPRINCS

UNION STATION HOT SPRINGS

UPPER FRISCO HOT SPRING

UPPER KLAWASI WARM SPRINGS

UPPER LOON CREEK HOT SPRINGS

UPPER SHELLBOURNE SPRING

UPPER WARM SPRINC

UPPER WARM SPRING

UPPER WAUNITA HOT SPRINGS

$$
\text { US BLM }
$$

UTAH FISH AND GAME DEPT

UTAH HOT SPRINGS

VALE HOT SPRINGS

VALLECITOS SPRING

VALLEJO WHITE SULPHUR SPRINCS

VALLEY SPRINGS

CO VALLEY VIEW HOT SPRINGS

VAUGHN HOT SPRINC

AZ VERDE HOT SPRINCS

$Y$ VERMILION SPRINCS

VEYO HOT SPRING

CA VICHY SPRINGS

CA VICKERS HOT SPRINGS

MT VICILANTE SPRING

WY VIOLET SPRINCS

UT VIRCIN RIVER SPRINC 
D VULCAN HOT SPRINCS

$$
\text { VYA SPRING }
$$

W UKINEK SPRINC

W R CARTER SPRINCS

WABUSKA HOT SPRINCS

CO WACON WHEEL GAP HOT SPRINGS

WAIWELAWELA POINT SPRINC

WALL CREEK WARM SPRINCS

WALLEYS HOT SPRINGS

WALTI HOT SPRINCS

WAMBOLT SPRINGS

CA WARD SPRINC

WARDROP HOT SPRING

WARDS HOT SPRINGS

WARFIID HOT SPRING

WARM DITCH SPRING

WARM LAKE SPRINCS

WARM MINERAL SPRINC

WARM MINERAL SPRINCS

AZ WARM SPRINC

WARM SPRINC

WARM SPRING

WARM SPRING

WARM SPRINC

WARM SPRINC

WARM SPRINC

WARM SPRING CREEK SPRINCS

AK WARM SPRINC NEAR PURCELL MOUNTAIN

WARM SPRINCS

WARM SPRINGS

GA WARM SPRINGS

ID WARM SPRINGS

MT WARM SPRINCS

NIT WARM SPRINGS

NM WARM SPRINCS

NV WARM SPRINCS
State

Spring name

OR WARM SPRINGS

WARM SPRINGS

WARM SPRINCS

WY WARM SPRINGS

WA WARM SPRINCS CANYON WARM SPRING

ID WARM SPRINGS CREEK HOT SPRINCS

CA WARM SULPHUR SPRINCS

HI WARM VAPOR

CA WARNER HOT SPRINC

OR WARNER VALLEY RANCH

UT WARNER VALLEY SPRINC

UT WASATCH HOT SPRINCS

WY WASHAKIE MINERAL HOT SPRINCS

WY WASHBURN HOT SPRINCS

CA WATERMAN HOT SPRING

ID WEATHERBY HOT SPRING

OR WEBERG HOT SPRING

NV WEDELL HOT SPRINGS

ID WEIR CREEK HOT SPRINCS

ID WEISER WARM SPRINCS

OR WELL SPRING

CO WELLSVILLE WARM SPRING

CA WENDEL HOT SPRINCS

MT WEST FORK SWIMMING HOLE

ID WEST PASS HOT SPRINC

NV WEST PINTO HOT SPRINC

WY WEST THUMB GEYSER BASIN

CA WEST VALLEY RESERVOIR HOT SPRINC

CA WHEELER SPRINCS

ID WHITE ARROW HOT SPRINC

WY WHITE CREEK CROUP

WY WHITE DOME GEYSER, SURPRISE POOL

NV WHITE HOUSE SPRINC

ID WHITE UCKS HOT SPRING

OR WHITE ROCK RANCH HOT SPRINC
State Spring name

NV WHITE ROCK SPRING

WHITE SULPHUR SPRINGS

WHITE SULPHUR SPRINCS
WHITE SULPHUR SPRINCS

MT WHITE SULPHUR SPRNO

CA WHITES POINT HOT SPRINCS

CA WHITMORE HOT SPRINGS

ID WHITTAKER WARM SPRINGS

CA WILBUR SPRINGS

NV WILD HORSE HOT SPRINCS

OR WILKERSONS HOT SPRINC

CA WILLETT HOT SPRINCS

NV WILLIAMS HOT SPRINC

ID WILLOW CREEK HOT SPRING

UT WILLOW SPRING

UT WILSON HEALTH SPRINCS

NV WILSON HOT SPRING

ID WILSON LAKE WARM SPRINC

WY WIND RIVER CANYON SPRINC

NV WINE CUP RANCH SPRINCS

CA WISTER MUD POT

MT WOLF CREEK HOT SPRINGS

ID WOLF HOT SPRINC

WY WOLF LAKE SPRINCS

UT WOOD SPRINGS

UT WOODHOUSE SPRING

ID WOODRUFF HOT SPRING

D WORSWICK HOT SPRINCS

CA WRENDEN HOT SPRINCS

ID YANDELL SPRINGS

CA YATES HOT SPRINGS

CA ZAMBONI HOT SPRINGS

CA ZIM ZIM SPRING

ID ZIMS RESORT HOT SPRINCS

NV ZOLEZZI SPRINC 


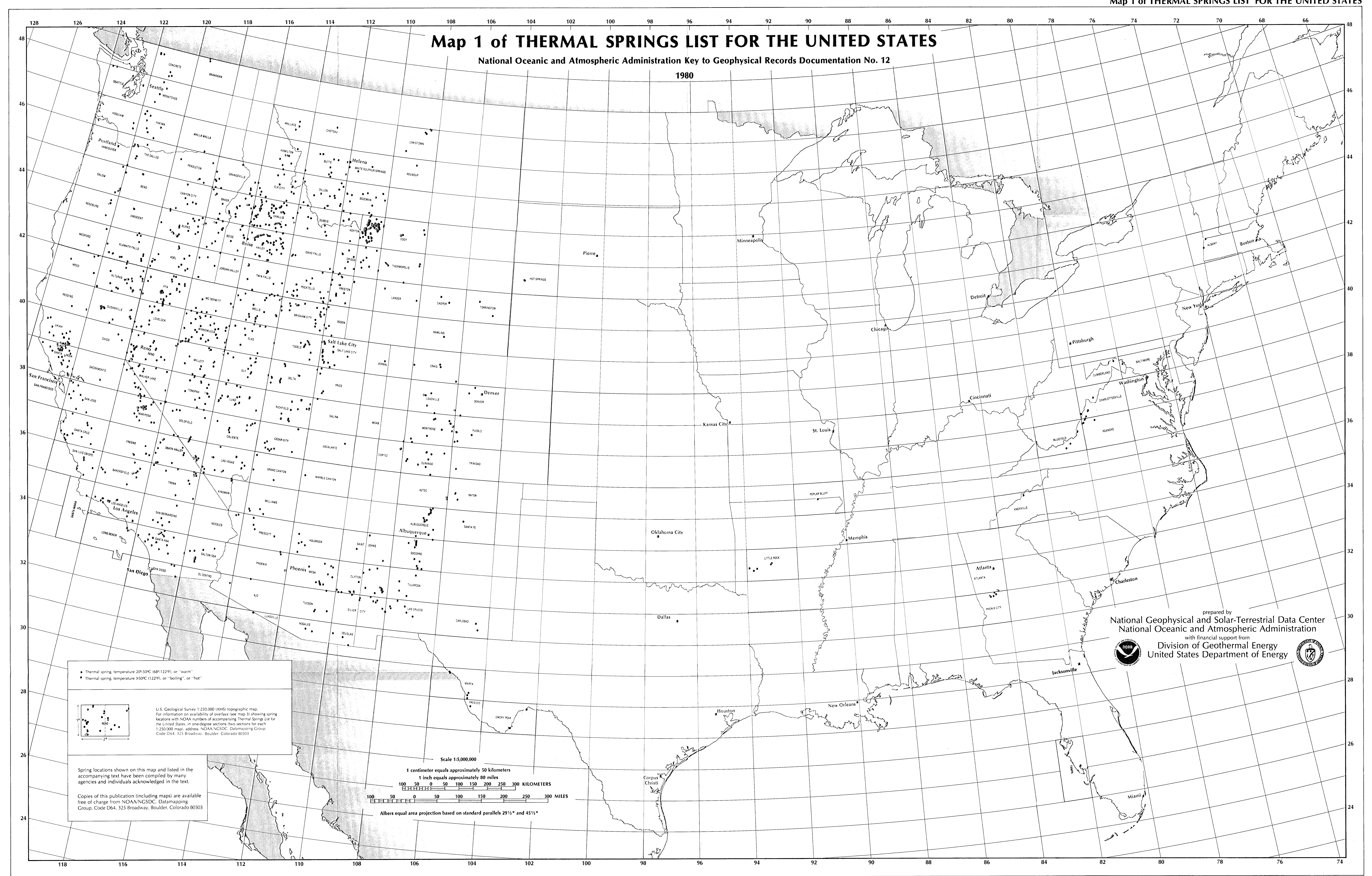


Map 2 of THERMAL SPRINGS LIST FOR THE UNITED STATES

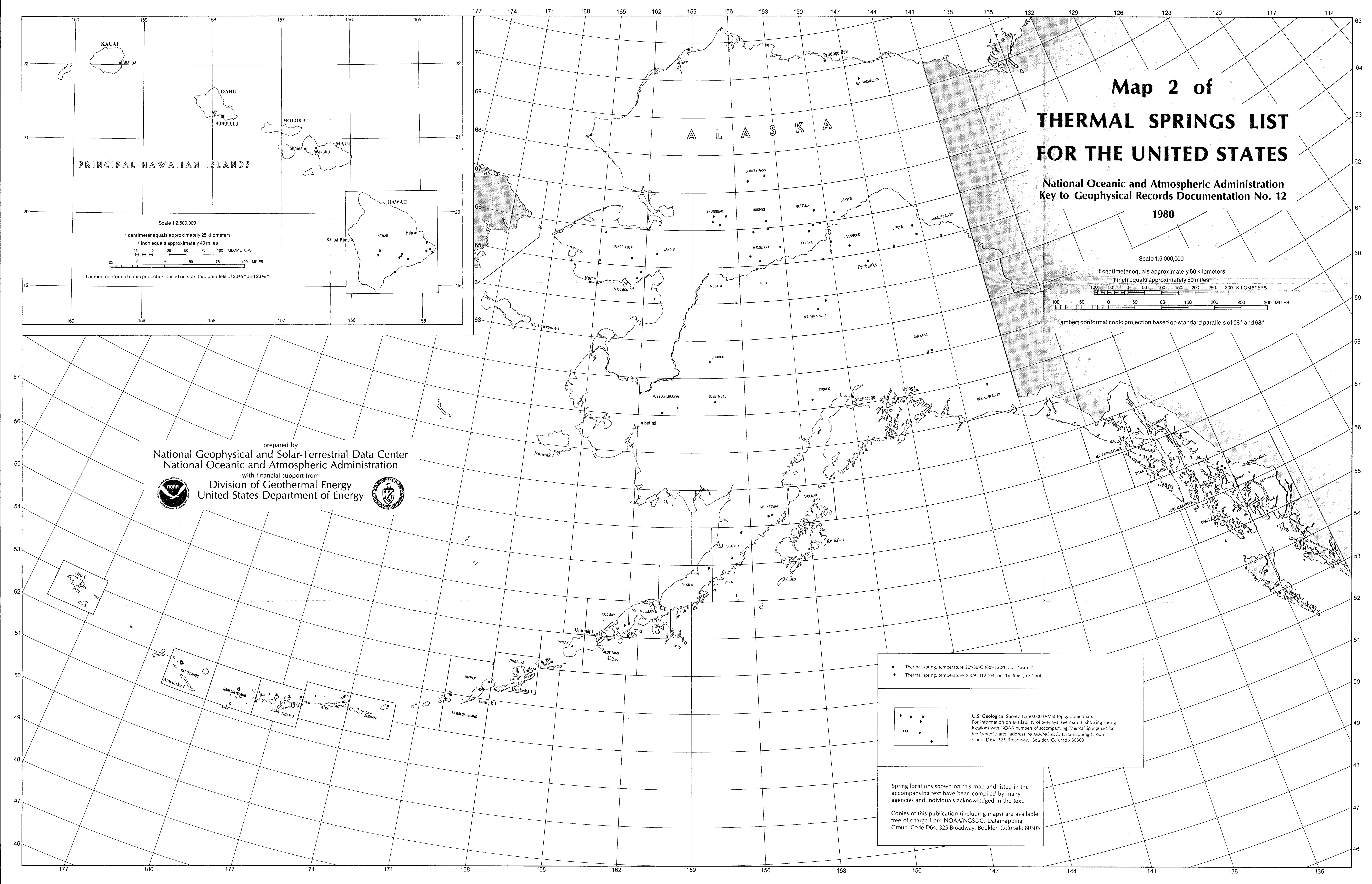


National Oceanic and Atmospheric Administration Key to Geophysical Records Documentation No. 12 